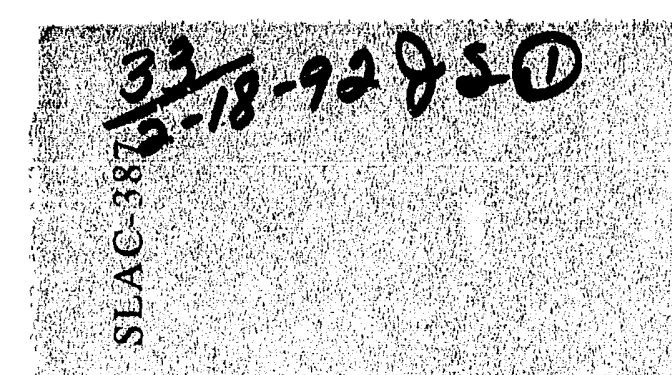

\title{
THE GENERATION AND ACCELERATION OF LOW EMITTANCE FLAT BEAMS FOR FUTURE LINEAR COLLIDERS
}

\section{Tor Q Raubentioimet}

SLAC-Report 387

November 1991

Prepared for the Department of Energy under contract number DE-AC03-76SF00515

DISTAIEUTION OF THIS DOCUMENT IS UNLIMITED

Stanford Linear Accelerator Center

Stanford Unversity - Stanford, California 
This document and the material and data contained therein, was developed under sponsorship of the United States Government. Neither the United States nor the Department of Energy, nor the Leland Stanford Junior University, nor their employees, nor their respective contractors, subcontractors, or their employees, makes any warranty, express or implied, or assumes any liability or responsibility for accuracy, completeness or usefulness of any information, apparatus, product or process disclosed, or represents that its use will not infringe privately-owned rights. Mention of any product, its manufacturer, or suppliers shall not, nor is it intended to, imply approval, disapproval, or fitness for any particular use. A royalty-free, nonexclusive right to use and disseminate same for any purpose whatsoever, is expressly reserved to the United States and the University. 
DE92 007395

\title{
THE GENERATION AND ACCELERATION OF LOW EMITTANCE FLAT BEAMS FOR FUTURE LINEAR COLLIDERS*
}

\author{
TOR O. RAUBENHEIMER \\ Stanford Linear Accelerator Center \\ Stanford University \\ Stanford, California 94309
}

\begin{abstract}
November 1991
Prepared for the Department of Energy under contract number DE-AC03-76SF00515
\end{abstract}

Printed in the United States of America. Available from the National Technical Information Service, U.S. Department of Commerce, 5285 Port Royal Road, Springfield, Virginia 22161.

* Ph.D. thesis

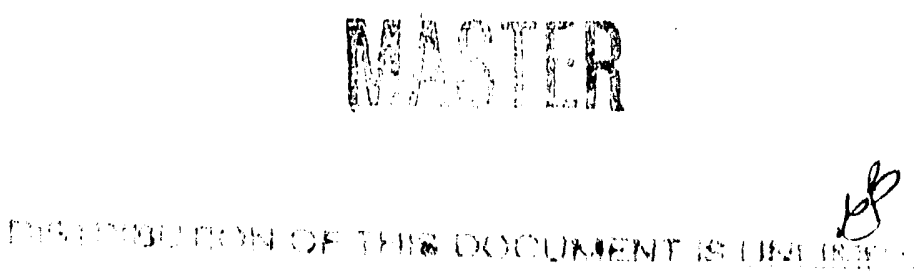




\begin{abstract}
Many future linear collider designs call for electron and positron beams with normalized rms torizontal and vertical emittances of $\gamma \epsilon_{x}=3 \times 10^{-6} \mathrm{~m}-\mathrm{rad}$ and $\gamma \epsilon_{y}=$ $3 \times 10^{-8} \mathrm{~m}$-rad; these are a factor of 10 to 100 below those observed in the Stanford Linear Collider. In this dissertation, we examine the feasibility of achieving beams with these very small vertical emittances. We examine the limitations encountered during both the generation and the subsequent acceleration of such low emittance beams. We consider collective limitations, such as wakefields, space charge effects, scattering processes, and ion trapping; and also low intensity limitations, such as anomalous dispersion, betatron coupling, and pulse-to-pulse beam jitter. In general, the minimum emittance in both the generation and the acceleration stages is limited by the transverse misalignments of the accelerator components. We describe a few techniques of correcting the effect of thesé erro.s, thereby easing the alignment tolerances by over an order of magnitude. Finally, we also calculate "fundamental" limitations on the minimum vertical emittance; these do not constrain the current designs but may prove important in the future.
\end{abstract}




\section{ACKNOWLEDGEMENTS}

I have burdened many people with my task of writing this dissertation. First, I would like to thank all of the people in the ATSP department and the SLC accelerator department for their support and collaboration.

Second, 1 want to thank two individuals who have served as both mentors and colleagues - Ron Ruth and Phil Morton. I have had many stimulating discussions with both of them over the years. In particular, Ron has provided direction, insight, and support for my work; he has been a wonderful teacher and a source of motivation.

Next, I would like to thank my thesis committee, Ewan Paterson, Bob Siemann, and Helmut Wiedemann. They have endured many over-extended deadlines and a very lengthy document (my apologies). Thankyou for the hours you have spent reading and the contributions you have made to better this work.

Finally, a special thanks to my close friends - Chark, Britt, Jaun, $\mathrm{A}^{2}, \mathrm{R} \& \mathrm{~A}$, Brad, Chackmo, Lenrow, Chach, and, of course, Kari. Thankyou for water fights, tequila, Joshua Tree - even a few broken bones. An even greater thanks to Krist and Wally for constant support and, more importantly, a sense of values. And lastly, I would like to thank Bamse and Freyja, whose only expectations have been dinner, and, most of all, I thank my best friend and companion, Susan Fernyak, for being herself. 


\section{TABLE OF CONTENTS}

1. THE GENERATION AND ACCELERATION OF

LOW EMITTANCE FLAT BEAMS . . . . . . . 1

1.1 INTRODUCTION . . . . . . . . . . . . . . . . 1

2. GENERATION OF SMALL VERTICAL EMITTANCE BEAMS . . . 5

2.1 INTRODUCTION . . . . . . . . . . . . . 5

2.1.1 Damping Rings . . . . . . . . . . . . . . . . . 6

2.1.2 Current Designs . . . . . . . . . . . . . . . . . 7

2.1 .3 Limitations . . . . . . . . . . . . . . . . . . . 8

2.2 SINGLE PARTICLE LIMITATIONS . . . . . . . . . . 9

2.2 .1 Introduction . . . . . . . . . . . . . . . . . . . 9

2.2.2 Preliminaries . . . . . . . . . . . . . . . . . . 11

2.2.3 Opening Angle Emittance . . . . . . . . . . . . . . . . . 19

2.2 .4 Vertical Dispersion . . . . . . . . . . . . . 23

2.2.5 Betatron Coupling . . . . . . . . . . . . . . . 33

2.2 .6 Synchrotron Motion . . . . . . . . . . . . . . 43

2.2 .7 Correction . . . . . . . . . . . . . . . . . . . 51

2.2.8 Distributions and Tolerances . . . . . . . . . . . 59

2.2 .9 Summary . . . . . . . . . . . . . . . . . . . . . . 69

2.2.10 Application to NLC Damping Ring . . . . . . . 70

2.3 COLleCtive Limitations . . . . . . . . . . . . . . 73

2.3.1 Intrabeam Scattering . . . . . . . . . . . . 73

2.3 .2 Ion Trapping . . . . . . . . . . . . . . . . . . . . 80

2.3.3 Space Charge . . . . . . . . . . . . . . . . . . . . 82

2.3.4 Wakefields: Static Effects . . . . . . . . . . . . 87

2.3.5 Wakefields: Dynamical Effects . . . . . . . . . . 93

2.3.6 Beam-Gas Scattering . . . . . . . . . . . . . . . . . 93

2.3.7 Lifetimes . . . . . . . . . . . . . . . . . . . . . . . . . 99

2.4 JITTER AND NOISE EFFECTS . . . . . . . . . . . 101

2.4 .1 Ground Motion . . . . . . . . . . . . . . . . 101

2.4.2 Power Supply Fluctuations . . . . . . . . . . . 102

2.4.3 High Frequency Jitter . . . . . . . . . . . . . 104

2.4.4 Feedback . . . . . . . . . . . . . . . 106

2.5 INJECTION MATCHING . . . . . . . . . . . . . . 107

2.6 GENERATION SUMMARY . . . . . . . . . . . . . . . . . 109 
3. ACCELERATION OF BEAMS WITH SMALL VERTICAL EMIT. 111

3.1 INTRODUCTION . . . . . . . . . . . . . 111

3.2 EQUATIONS OF MOTION AND PROJECTED EMIT. - 113

3.2.1 Equations of Motion . . . . . . . . . . . . . . 113

3.2.2 Projected Emittance . . . . . . . . . . . . . . 115

3.3 FILAMENTATION AND TRANSVERSE MATCHING . . 117

3.3.1 Filamentation and Natural $\beta$ Functions . . . . . . 117

3.3.2 Matching Tolerances . . . . . . . . . . . . . . 123

3.4 PRIMARY SOURCES OF DILUTION . . . . . . . . 125

3.4.1 Dispersive Errors . . . . . . . . . . . . . . 125

3.4.2 Transverse Wakefields . . . . . . . . . . . . . 131

3.4.3 RF Deflections . . . . . . . . . . . . . . . . 141

3.4.4 Distributions . . . . . . . . . . . . . . . . . . 142

3.5 TRAJECTORY CORRECTION . . . . . . . . . . . 144

3.5.1 Introduction . . . . . . . . . . . . . . 144

3.5.2 DF Correction . . . . . . . . . . . . . . . . . 145

3.5.3 DF Error Analysis . . . . . . . . . . . . . . . 147

3.5.4 DF Simulations . . . . . . . . . . . . . . . . 156

3.5.5 WF Correction and Simulations . . . . . . . . 164

3.5.6 Further Extensions . . . . . . . . . . . . . . . 170

3.5.7 Tolerances with DF/WF . . . . . . . . . . . 170

3.5.8 Discussion . . . . . . . . . . . . . . . . . . 172

3.6 ADDITIONAL CORRECTION METHODS . . . . . . 174

3.6.1 RF Deflecting Cavities . . . . . . . . . . . . 174

3.6.2 Trajectory Bumps . . . . . . . . . . . . . . . 174

3.7 MAGNETIC FIELD TOLERANCES . . . . . . . . . 178

3.7.1 Quadrupole Tolerances . . . . . . . . . . . 179

3.7.2 Skew Quadrupole Tolerances . . . . . . . . . . 180

3.8 PULSE-TO-PULSE JITTER . . . . . . . . . . . . . . . 182

3.8.1 Injection Jitter . . . . . . . . . . . . . . . . . 183

3.8.2 Random Jitter . . . . . . . . . . . . . . . . . . 183

3.8.3 Ground Motion . . . . . . . . . . . . . . . . 185

3.8.4 Power Supply Fluctuations . . . . . . . . . . . 190

3.8.5 Feedback . . . . . . . . . . . . . . . . . 192

3.9 ADDITIONAL EFFECTS . . . . . . . . . . . . . . 194

3.9.1 Space Charge . . . . . . . . . . . . . . . . . 194 
3.9.2 Radiation . . . . . . . . . . . . . . . . . . 196

3.9.3 Scattering . . . . . . . . . . . . . . . . . . . . . 198

3.10 ACCELERATION SUMMARY . . . . . . . . . . 200

4. CONCLUDING REMARKS . . . . . . . . . . . . . . . . . . 203

A. Appendix: BEAM DYNAMICS: SURVEY . . . . . . . . . . . 205

A.1 Coordinate System . . . . . . . . . . . . . . 205

A.2 Transverse Motion . . . . . . . . . . . . . . 205

A.3 Longitudinal Motion . . . . . . . . . . . . . . . . 213

A.4 Radiation Damping . . . . . . . . . . . . . 215

A.5 Quantum Excitation . . . . . . . . . . . . 216

A.6 Damping Ring Parameters . . . . . . . . . . . . . . . . . 219

B. Appendix: NLC DAMPING RING DESIGN . . . . . . . . . . . 223

B.1 Original Design Goals . . . . . . . . . . . . . . . . . 223

B.2 Current Design . . . . . . . . . . . . . . . . . . . . 224

B.3 Future Modifications . . . . . . . . . . . . . . . . . 228

B.4 Limitations . . . . . . . . . . . . . . . . . . . . . . 228

C. Appendix: COUPLING DERIVATIONS . . . . . . . . . . . 231

C.1 Independence of Contributions to $\epsilon_{y} \ldots \ldots \ldots$. . . . . . 231

C.2 Derivation of Eqs. (2.2.65) and (2.2.107) . . . . . . . . 232

D. Appendix: NLC LINEAR ACCELERATORS . . . . . . . . . . 235

D.1 Basic Scaling . . . . . . . . . . . . . . . . . . . . 235

D.2 Preliminary Linac . . . . . .

D.3 Main Linac . . . . . . . . . . . . . . . . . . . . . . . 240

REFERENCES . . . . . . . . . . . . . . . . . . . 242 


\section{TABLES}

1. Parameters of linear collider designs. "

2. Future linear collider damping ring parameters. 7

3. The effect of different alignment errors. 15

4. Simulations of the vertical dispersion. 30

5. Simulations of the betatron coupling. 40

6. Longitudinal parameters for the SLC NDR and the ALS. 48

7. Simulations of global correction of vertical dispersion. 58

8. Simulations of local correction of vertical dispersion. 58

9. Simulations of global correction of betatron coupling. 59

10. Emittance due to alignment errors in the NLC damping ring. 71

11. Tolerances for the NLC damping ring. 71

12. Results of DF simulations in the $250 \mathrm{GeV}$ NLC linac. 157

13. Results of WF simulations in the $250 \mathrm{GeV}$ NLC linac. 166

14. Simulations of "non-dispersive" bumps in the NLC linac. 177

15. Tolerances in the $500 \mathrm{GeV}$ NLC main linac. 201

16. Jitter tolerances in the $500 \mathrm{GeV}$ NLC main linac. 201

17. Basic parameters of the SLC and NLC damping rings. 224

18. NLC damping ring parameters. 225

19. NLC damping ring transverse parameters. 225

20. NLC ring longitudinal parameters. 226

21. NLC ring magnet and wiggler parameters. 227

22. Comparison of basic cells for NLC ring. 227

23. Average beta functions for various cell phase advances. 238

24. Parameters for the preliminary NLC linac. 239

25. Parameters for the main NLC linac. 240 


\section{ILLUSTRATIONS}

1. Schematic of the NLC, a future linear collider. 3

2. Vertical dispersion versus $\xi_{y}$ for an uncorrected orbit. 31

3. Vertical dispersion versus $\xi_{y}$ for a corrected orbit. 31

4. Vertical emittance due to dispersion in the NDR. 33

5. Vertical emittance due to dispersion in the ALS. 33

6. Vertical emittance due betatron coupling in the NDR. 42

7. Vertical emittance due betatron coupling in the ALS. 42

8. Moments of the distribution of the emittance. 60

9. Distributions of the emittance due to dispersion. 63

10. $95 \%$ confidence level for $\epsilon_{y}$ due to dispersion. 64

11. Distributions of the emittance due to betatron coupling. 67

12. $95 \%$ confidence level for $\epsilon_{y}$ due to betatron coupling. 67

13. Emittance due to IBS versus aspect ratio. 76

14. Emittance versus time with IBS. 78

15. Bands of stable (trapped) ion masses. 81

16. Direct space charge field. 83

17. Particle distribution with beam-gas scattering. 97

18. Touschek function $D\left(P_{x}\right) . \quad 100$

19. Dispersive filamentation of a coherent betatron oscillation. 118

20. Chromatic filamentation of a mismatched beam. 119

21. Filamentation of a beta mismatch. 121

22. Filamentation in a transport line. 121

23. Dispersive filamentation of an oscillation in the $500 \mathrm{GeV}$ linac. $\quad 129$

24. Schematic of one-to-one trajectory correction. 130

25. Emittance dilution with BNS damping in the $500 \mathrm{GeV}$ NLC linac. 135

26. Schematic of trajectory correction with wakefields. 137 
27. Wakefield dilution due to a corrected trajectory. 138

28. Emittance dilution due to a corrected trajectory versus $\psi_{c}$. 139

29. Actual trajectory in the NLC after DF correction. 158

30. BPM measurements in the NLC after DF correction. 158

31. Difference orbit in the NLC after DF correction. 159

32. Phase-space at the end of the NLC after one-to-one correction. $\quad 160$

33. Phase-space at the end of the NLC after DF correction. 160

34. DF correction versus the energy change $\Delta E / E$. 162

35. DF correction versus the BPM precision errors. 162

36. Trajectory after WF correction in the $250 \mathrm{GeV}$ NLC linac. 168

37. Emittance after WF correction in the $250 \mathrm{GeV}$ NLC linac. 168

38. Phase space linac after 1-to-1, DF, and WF correction. 169

39. 1-to-1 and DF correction versus the rms misalignment magnitude. 171

40. Frequency spectrum of trajectory after 1-to-1 and WF correction. 173

41. A "non-dispersive" bump in the $250 \mathrm{GeV} \mathrm{NLC} \mathrm{linac.} 176$

42. Blow-up of Fig. 41.

43. Three lowest order ground motion resonances. 186

44. Response to ground motion in the $500 \mathrm{GeV}$ NLC linac. 189

45. Frequency response for SLC feedback system: $f_{\text {rep }}=60 \mathrm{~Hz}$. 193

46. Transient response for SLC feedback system: $f_{\text {rep }}=60 \mathrm{~Hz}$. 193

47. Common magnets used in accelerators. 206

48. Phase space of a mismatched beam. 212

49. Schematic of the NLC damping ring. 224

50. Lattice functions for the basic FODO cell. 236

51. Wakefield for $11.4 \mathrm{GHz}$ structure. 241 


\title{
SYMBOLS
}

\author{
$\alpha_{m} \quad$ momentum compaction: $\alpha_{m} \equiv d C / C / d E / E$, Eq. (A.3.4). \\ $\alpha_{x, y, z} \quad$ transverse and longitudinal damping rates, Appendix A.4, or, \\ lattice (Twiss) parameters, Appendix A.2. \\ $\alpha_{x, y}^{\star} \quad$ beam parameters, Eq. (A.2.20). \\ $\beta_{x, y} \quad$ lattice (Twiss) parameters, Appendix A.2. \\ $\beta_{x, y}^{\star} \quad$ beam parameters, Eq. (A.2.20). \\ b vacuum chamber radius, Section 2.3.4. \\ c speed of light: $c=3 \times 10^{8} \mathrm{~m} / \mathrm{s}$. \\ $c_{n} \quad$ Fourier coefficient of the closed orbit correlation, Eq. (2.2.20). \\ C storage ring circumference. \\ $C_{q} \quad$ quantum coefficient: $C_{q}=3.84 \times 10^{-13} \mathrm{~m}$, Appendix A.5. \\ $\delta \quad$ relative energy deviation: $\delta \equiv\left(p-p_{0}\right) / p_{0}$, Appendix A.2. \\ $\epsilon_{x, y, z} \quad$ transverse and longitudinal beam emittances, Eq. (A.2.18). \\ $f \quad$ local chromaticity: $f \equiv K_{2} \eta_{x}-K_{1}$, Section 2.2.4. \\ $f_{n} \quad$ coefficient for synchro-betatron coupling, Section 2.2.6.1. \\ $f_{\mathrm{CL}} \quad$ location of $95 \%$ confidence level in units of the expected value, \\ Section 2.2.8. \\ $\mathcal{F} \quad$ measure of emittance dilution, Eq. (3.4.4). \\ $\gamma \quad$ relativistic factor. \\ $\gamma_{x, y} \quad$ lattice (Twiss) parameters, Appendix A.2. \\ $\gamma_{x, y}^{\star} \quad$ beam parameters, Eq. (A.2.20). \\ $g \quad$ distribution function for emittances, Section 2.2.8. \\ $y_{n} \quad$ coefficient for synchro-betatron coupling, Section 2.2.6.1. \\ $\mathcal{G} \quad$ accelerating gradient: $\mathcal{G} \equiv d \gamma / d s$. \\ $G \quad$ inverse bending radius of main bending field, Eq. (A.2.1).
}




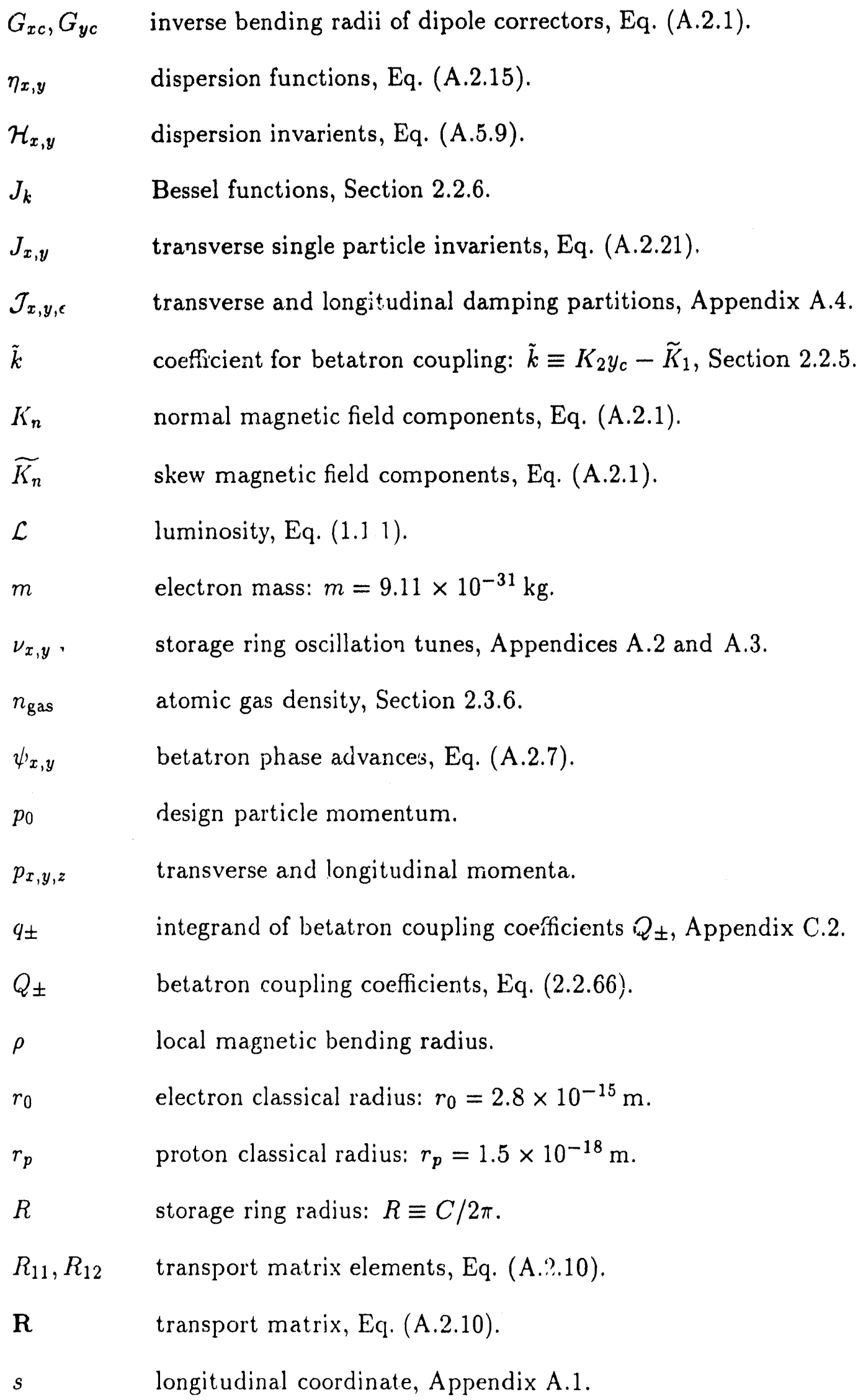


$S_{ \pm} \quad$ synchro-betatron coupling coefficients, Eq. (2.2.86).

$\sigma$ cross section for scattering, or, conductivity of vacuum chamber: for copper $\sigma=5.8 \times 10^{7} \mathrm{mhos} / \mathrm{m}$.

$\sigma_{x, y, z, \epsilon} \quad$ rms beam sizes for transverse, longitudinal and energy deviation.

$\tau_{x, y, z} \quad$ transverse and longitudinal damping times, Appendix A.4.

$T_{0} \quad$ revolution period of storage ring.

$\Omega \quad$ synchrotron oscillation frequency, or, solid angle.

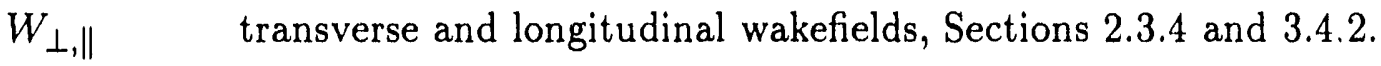

$\xi_{x, y} \quad$ transverse chromticities: $\xi_{x, y} \equiv d \nu / d p / p$, Eq. (A.2.17).

$x, y, z \quad$ transverse and longitudinal coordinates, Appendix A.1.

$x_{\beta}, y_{\beta} \quad$ betatron component of $x$ and $y$ motion, Eq. (A.2.6).

$x_{c}, y_{c} \quad$ horizontal and verical closed orbit, Eq. (A.2.13).

$x_{m}, y_{m} \quad$ transverse misalignments.

$Z_{0} \quad$ impedance of free-space: $Z_{0}=377 \Omega$. 
$G_{x c}, G_{y c} \quad$ inverse bending radii of dipole correctors, Eq. (A.2.1).

$\eta_{x, y} \quad$ dispersion functions, Eq. (A.2.15).

$\mathcal{H}_{x, y} \quad$ dispersion invarients, Eq. (A.5.9).

$J_{k} \quad$ Bessel functions, Section 2.2.6.

$J_{x, y} \quad$ transverse single particle invarients, Eq. (A.2.21).

$\mathcal{J}_{x, y, \epsilon} \quad$ transverse and longitudinal damping partitions, Appendix A.4.

$\tilde{k} \quad$ cocfficient for betatron coupling: $\tilde{k} \equiv K_{2} y_{c}-\widetilde{K}_{1}$, Section 2.2.5.

$K_{n} \quad$ normal magietic field components, Eq. (A.2.1).

$\widetilde{K_{n}} \quad$ skew magnetic field components, Eq. (A.2.1).

$\mathcal{L} \quad$ luminosity, Eq. (1.1.1).

$m \quad$ electron mass: $m=9.11 \times 10^{-31} \mathrm{~kg}$.

$\nu_{x, y, s} \quad$ storage ring oscillation tunes, Appendices A.2 and A.3.

$n_{\text {gas }} \quad$ atomic gas density, Section 2.3.6.

$\psi_{x, y} \quad$ betatron phase advances, Eq. (A.2.7).

$p_{0} \quad$ design particle momentum.

$p_{x, y, z} \quad$ transverse and longitudinal momenta.

$q_{ \pm} \quad$ integrand of betatron coupling coefficients $Q_{ \pm}$, Appendix C.2.

$Q_{ \pm} \quad$ betatron coupling coefficients, Eq. (2.2.66).

$\rho \quad$ local magnetic bending radius.

$r_{0} \quad$ electron classical radius: $r_{0}=2.8 \times 10^{-15} \mathrm{~m}$.

$r_{p} \quad$ proton classical radius: $r_{p}=1.5 \times 10^{-18} \mathrm{~m}$.

$R \quad$ storage ring radius: $R \equiv C / 2 \pi$.

$R_{11}, R_{12} \quad$ transport matrix elements, Eq. (A.2.10).

R transport matrix, Eq. (A.2.10).

$s \quad$ longitudinal coordinate, Appendix A.1. 
$S_{ \pm} \quad$ synchro-betatron coupling coefficients, Eq. (2.2.86).

$\sigma$

cross section for scattering, or, conductivity of vacuum chamber: for copper $\sigma=5.8 \times 10^{7} \mathrm{mhos} / \mathrm{m}$.

$\sigma_{x, y, z, \epsilon} \quad \mathrm{rms}$ beam sizes for transverse, longitudinal and energy deviation.

$\tau_{x, y, z} \quad$ transverse and longitudinal damping times, Appendix A.4.

$T_{0} \quad$ revolution period of storage ring.

$\Omega \quad$ synchrotron oscillation frequency, or, solid angle.

$W_{\perp, \|} \quad$ transverse and longitudinal wakefields, Sections 2.3.4 and 3.4.2.

$\xi_{x, y} \quad$ transverse chromticities: $\xi_{x, y} \equiv d \nu / d p / p$, Eq. (A.2.17).

$x, y, z \quad$ transverse and longitudinal coordinates, Appendix A.1.

$x_{\beta}, y_{\beta} \quad$ betatron component of $x$ and $y$ motion, Eq. (A.2.6).

$x_{c}, y_{c} \quad$ horizontal and verical closed orbit, Eq. (A.2.13).

$x_{m}, y_{m} \quad$ transverse misalignments.

$Z_{0} \quad$ impedance of free-space: $Z_{0}=377 \Omega$. 


\section{CHAPTER 1 \\ THE GENERATION AND ACCELERATION OF LOW EMITTANCE FLAT BEAMS}

In this dissertation, we will examine the generation and subsequent acceleration of electron and positron beams with very small vertical emittances. Currently, many groups around the world are designing the "next generation" of $e^{+} / e^{-}$linear colliders; the first generation is the Stanford Linear Collider (SLC) at SLAC. The future accelerators are designed to have center-of-mass energies of $\frac{1}{2}$ to $2 \mathrm{TeV}$.

To perform useful measurements, these accelerators need to have very high luminosities; the luminosity multiplied by the cross section of interest specifies the experimental event rate. The linear colliders are being designed for luminosities of $10^{33}$ to $10^{34} \mathrm{~cm}^{-2} \mathrm{sec}^{-1}$. This is one-to-two orders of magnitude higher than that currently achieved in colliding beam machines, and many orders of magnitude higher than that achieved by the SLC.

The luminosity depends upon the density of the colliding beams and the degree of overlap. Assuming that the beams fully overlap, the luminosity can be written:

$$
\mathcal{L}=\frac{N_{-} N_{+} n_{b} f_{\mathrm{rep}}}{4 \pi \sigma_{x} \sigma_{y}} H_{D}
$$

where we have assumed that the bearns have gaussian transverse distributions with rms dimensions of $\sigma_{x}$ and $\sigma_{y}$. In addition, $N_{-}$and $N_{+}$are the number of electrons and positrons per bunch, $n_{b}$ is the number of bunches per batch, $f_{\text {rep }}$ is the batch repetition rate, and $H_{D}$ is the pinch enhancement factor.

To achieve the required luminosity, many future linear colliders designs are striving for very small spot sizes at the interaction point (IP); parameters of a number of the designs are listed in Table i. $^{\star \dagger}$ The spot size is described by two parameters: the beam emittance, which is a measure of the beam's phase space volume, and

* The parameters are in $a$ state of flux and, in addition, many laboratories have multiple sets of parameters. Thus, we have chosen a representative, but not necessarily current or exhaustive, set.

$\uparrow$ There are two designs (not listed) that have much larger spot sizes: the superconducting TESLA design and the high current DESY design. These machines achieve the necessary luminosity by having many bunches. 
Table 1. Parameters of linear collider designs.

\begin{tabular}{|c|c|c|c|c|c|}
\hline & SLC $^{[128]}$ & NLC $^{[127]}$ & JLC $^{[127]}$ & CLIC $^{[127]}$ & DESY $^{[57]}$ \\
\hline C-O-M Energy $[\mathrm{TeV}]$ & 0.1 & 1.0 & 1.0 & 2.0 & 0.5 \\
\hline Luminosity $\left[10^{33} \mathrm{~cm}^{-2} \mathrm{sec}^{-1}\right]$ & 0.006 & 10 & 6 & 12 & 4.1 \\
\hline$e^{+} / e^{-}$per bunch $\left[10^{10}\right]$ & 5.0 & 2.0 & 1.0 & 0.5 & 0.7 \\
\hline Number bunches & 1 & 10 & 10 & 11 & 172 \\
\hline Repetition rate $[\mathrm{Hz}]$ & 180 & 180 & 200 & 1700 & 50 \\
\hline$\sigma_{x}[\mathrm{~nm}]$ & 1600 & 220 & 200 & 60 & 169 \\
\hline$\sigma_{y}[\mathrm{~nm}]$ & 1600 & 2.5 & 1.7 & 12 & 5.5 \\
\hline$\sigma_{z}[\mu \mathrm{m}]$ & 1000 & 100 & 76 & 200 & 200 \\
\hline Emittance: $\gamma \epsilon_{x}\left[10^{-6} \mathrm{~m}-\mathrm{rad}\right]$ & 16 & 3 & 3 & 3 & 4 \\
\hline$\gamma \epsilon_{y}\left[10^{-6} \mathrm{~m}\right.$-rad $]$ & 16 & 0.03 & 0.03 & 1 & 0.04 \\
\hline
\end{tabular}

the beta function which depends upon the focusing structure of the accelerator. In particular, the spot size can be written:

$$
\sigma_{x, y}=\sqrt{\beta_{x, y} \epsilon_{x, y}}
$$

where $\beta_{x, y}$ is the beta function and $\epsilon_{x, y}$ is the projected emittance. To reduce the spot size, we need to reduce both the beta function and the beam emittance at the IP; this is the driving force behind small emittance beams.

To get a small emittance beam at the IP, the beam is created at low energy and then (very carefally) accelerated to the final energy; Figure 1 shows a schematic of the proposed linear collider. The main components are: damping rings to generate the very low emittance electron and positron beams, bunch compressors to decrease the length of the particle bunches, linear accelerators to accelerate the beams, and final focus regions to demagnify the beams to very small sizes.

In this dissertation, we will examine the limitations and the tolerances associated with both the generation and the subsequent acceleration of beams with very small vertical emittances; we will not consider problems encountered in the bunch compressors or the final focus and we neglect all of the issues associated with having multiple closely spaced bunches. Although much of the physics is the same in both the damping rings and the linear accelerators, the relative importance differs; 


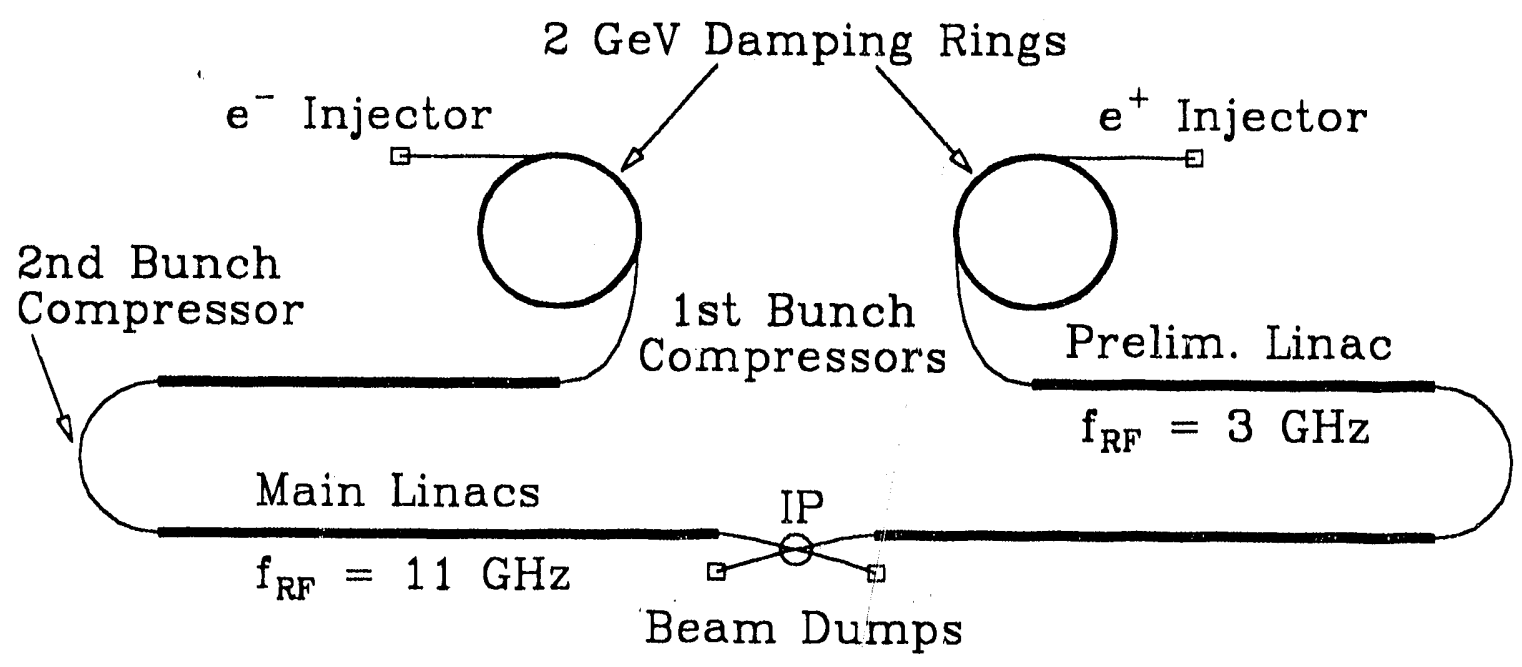

Fig. 1. Schematic of the NLC, a future linear collider.

the primary sources of dilution in the damping rings are non-conservative processes while in the linacs the main dilutions are due to conservative dilutions and pulse-topulse jitter. Thus, we will consider each region separately, breaking this dissertation into two main sections.

Specifically, in Section 2, we will discuss determination of the vertical emittance in the damping rings. Here, we will calculate the emittance dilutions due to:

- Opening angle of radiation

- Betatron coupling

- Intrabeam scattering

- Space charge field

- Beam-gas scattering

- Ground motion

- High frequency jitter
- Vertical dispersion

- Synchro-betatron coupling

- Ion trapping

- Wakefields

- Lifetime limits

- Power supply fluctuations

- Injection matching.

And, in Section 3, we will discuss the vertical emittance dilutions in the linear accelerators, calculating the dilutions due to:

- Matching and filamentation

- Transverse wakefields

- Betatron coupling

- Synchrotron radiation
- Vertical dispersion

- RF deflections

- Space charge field

- Beam-gas scattering 
- Ground motion

- pulse-to-pulse jitter.

We have attempted to make a complete analysis of the effects that determine the vertical emittance in the damping rings and the effects that can dilute the emittance during the subsequent acceleration. Much of this work is analysis of well known phenomena, in a new regime. However, some of it is actually new analysis, while the remainder is a compilation of others work, sometimes with minor corrections or extensions. We attempt to clarify this with extensive references.

Finally, it is worth making two remarks to prevent potential confusion: first, the references are organized in alphabetical order and thus the reference numbers do not appear sequentially. Second, most equations are written in a form that does not depend upon the explicit system of units, but, whenever the choice of units is important, the MKS system is used. 


\section{CHAPTER 2 \\ GENERATION OF SMALL VERTICAL EMITTANCE BEAMS}

In this chapter, we will discuss the generation of $e^{+} / e^{-}$beams with the very small vertical emittances required by many future linear collider designs; parameters are listed in Table 1. Many designs have specified normalized vertical emittances of roughly $3 \times 10^{-8} \mathrm{~m}$-rad. This is over one order of magnitude smaller than that specified in any of the current colliding beam storage rings or synchrotron light sources.

In general, the electron beam originates at an "injector" while the positron beam is created by colliding an electron beam with a target; the positrons are collected from the resulting electron-positron pairs. At this time, electron injectors are not able to generate beams with the necessary emittances and currents. Currently, advanced injectors, using $R F$ guns with photo-cathodes, are able to generate beams with $\gamma \epsilon_{\perp} \gtrsim 10^{-5} \mathrm{~m}$-rad at currents of $2 \times 10^{10} e^{-}$per pulse. ${ }^{[118,131]}$ Theoretical considerations suggest that an order of magnitude improvement is possible, but not the two-to-three orders of magnitude that are required. Furthermore, although some new techniques have been suggested, ${ }^{[11]}$ there are no known techniques of producing positrons with comparable emittances; usually conventional positron systems generate beams with emittances of $\gamma \epsilon_{\perp} \sim 10^{-2}-10^{-3} \mathrm{~m}$-rad. Thus, the beam emittances must be damped to decrease the six-dimensional emittance after the beams have been created.

Liouville's theorem can be used to show that the phase space density of an infinite number of particles is conserved in the absence of inter-particle and dissipative forces. This does not leave many avenues to damp the beam. Currently, three techniques have been developed to damp high energy charged particle beams: stochastic cooling, electron cooling, and radiation damping. The first two techniques are typically used on protons and ions where the radiation damping is ineffective. Both processes are much too slow to be useful for a future linear collider.

The other technique, radiation damping, damps the beam by causing the particles to radiate. The phase space density is damped because the radiation acts as a dissipative force. Usually, one uses synchrotron radiation to perform the damping, although any form of incoherent radiation, such as Cherenkov and transition radiation, will also damp the beam. 
It is important to realize that the radiation must be incoherent to damp the beam; each particle must radiate independently. If one were to write a Hamiltonian for the system, the coordinates and momenta of each photon need to be included; the phase space density of the beam is damped at the expense of the photon phase space. In contrast, if there is coherent or stimulated radiation, there is a definite phase relationship between the photons. In this case, one can describe the radiation as an interaction with an external field and the phase space density of the beam is conserved.

Explicitly, stimulated devices such as Free Electron Lasers (FELs), klystrons, gyrotrons, etc., do not damp the beam. FELs and klystrons bunch the beam longitudinally, but they increase the energy spread within the bunches. Similarly, gyrotrons bunch the beam in transverse position (phase) but this is done at the expense of the transverse momenta. This is also true of the Cyclotron Maser Cooling technique suggested in Ref. 59. This device may generate substantial radiation, but it will not damp the beam; the author has confused radiation power with damping and he did not examine the effect on the beam phase space.

\subsubsection{Damping Rings}

Although the radiation damping process is faster than other techniques, it is still relatively slow. Thus, the beams are damped while they are stored in a damping ring. As the highly relativistic particles are directed around the circumference of the ring, they emit synchrotron radiation. This incoherent radiation has two competing effects; it is a source of damping and a source of excitation.

The damping occurs because the synchrotron radiation acts as a frictional force, decreasing the particle's momentum deviation from the design momentum, while the excitation occurs because of the quantum nature of the radiation. Specifically, the radiation of a photon changes the particle's energy and gives a small transverse kick that depends upon the opening angle of the radiation. The transverse kicks due to the opening angle directly change the amplitude of the particle's betatron motion, and thereby the bunch's emittance, while the change in energy, due to the radiation, has a more subtle effect. The particle executes betatron oscillations about a closed orbit in the ring. Since this closed orbit depends upon the particle energy, the radiation of a photon increases the rms amplitude of the betatron motion by displacing the closed orbit relative to the particle's position. [104] 
Table 2. Damping ring parameters. [134] $^{\text {(3) }}$

\begin{tabular}{|c|c|c|c|c|c|}
\hline & SLC $^{(22,122)}$ & CLIC $^{(38]}$ & JLC $^{(119]}$ & NLC $^{(00)}$ & DESY $^{(22)}$ \\
\hline Energy $[\mathrm{GeV}]$ & 1.15 & 2.0 & 1.54 & 1.8 & 3.15 \\
\hline Circumference [meters] & 35 & $162+$ ins. & 180 & 155 & 650 \\
\hline Rep. rate $[\mathrm{Hz}]$ & 180 & 1700 & 200 & 360 & 50 \\
\hline Current/bunch $\left[10^{10} e^{+} / e^{-}\right]$ & 5 & 0.5 & 1 & 1.4 & 0.7 \\
\hline Number of bunches & 2 & $22 \times 10$ & $8 \times 10$ & $10 \times 10$ & 172 \\
\hline Injected $\gamma \epsilon_{x, y}\left[10^{-3} \mathrm{~m}-\mathrm{rad}\right]$ & 10 & - & 3 & 3 & - \\
\hline Extracted $\gamma \epsilon_{x}\left[10^{-6} \mathrm{~m}\right.$-rad] & 16 & 3 & 3 & 3 & 4.1 \\
\hline Coupling $\epsilon_{x} / \epsilon_{y}$ & 1 & 3 & 100 & 100 & 100 \\
\hline
\end{tabular}

In a storage ring these two competing effects lead to an equilibrium beam size and emittance. When designing a damping ring, one strives to maximize the damping while keeping the equilibrium emittance small. A review of the basic dynamics in an $e^{+} / e^{-}$ring is presented in Appendix A along with a more detailed discussion of damping rings and the basic scaling of damping ring parameters.

\subsubsection{Current Designs}

The main parameters of four damping ring designs for high energy linear colliders are compared with the SLC damping rings in Table 2; the NLC design is described in greater detail in Appendix B. Although these rings are each optimized for the respective linear colliders, there are two primary differences between the future designs and the SLC damping rings: first, the future rings are striving for very small emittances, especially the JLC, NLC, and DESY designs which call for extracted vertical emittances of $\gamma \epsilon_{y}=3 \sim 4 \times 10^{-8} \mathrm{~m}$-rad; this is a factor of 500 smaller than the SLC design.

Second, the future designs plan to provide much more damping than the SLC damping rings. For example, in the NLC design, the injected vertical emittance is damped twice as much as in the SLC rings while operating at a faster repetition rate. To achieve the necessary damping, the rings have larger circumferences, allowing multiple batches of bunches to be damped at once. In addition, the rings operate at slightly higher energies and use smaller angle bends to further increase the damping 
while maintaining small equilibrium emittances. Finally, some of the designs plan to supplement the damping with damping wigglers.

\subsubsection{Limitations}

As mentioned, there are two primary differences between the SLC damping rings and the future designs: the damping requirements and the emittances. In principal, the damping requirement is not really a limitation; one can always cascade multiple rings to decrease the requirements. The difficulty, of course, is in arriving at an elegant solution.

In contrast, the very small vertical emittance, that many designs require, may prove more difficult to achieve; we will examine the feasibility of this requirement. In the next sections, we will analyze the limitations on the vertical emittance and the aspect ratio $\epsilon_{x} / \epsilon_{y}$. In Section 2.2 , we discuss the single particle limitations; these are primarily due to transverse betatron coupling and the vertical dispersion. Next, in Section 2.3, we examine the collective limitations: intrabeam scattering, ion trapping, direct space charge effects, and wakefields. In addition, we will discuss limitations due to beam-gas scattering and the beam lifetimes. In Sections 2.4 and 2.5, we calculate the effect of jitter sources and the required injection matching; matching the extracted beam is discussed in Section 3.3.2. Finally, in Section 2.6, we summarize our results.

Of course, the vertical emittance is not the only limitation facing these future damping rings and, for completeness, we briefly describe a few of the other problems in Appendix B. 


\section{CHAPTER $\quad 2.2$ SINGLE PARTICLE LIMITATIONS}

\subsubsection{Introduction}

In this chapter, we discuss low current effects that contribute to both the vertical emittance and the vertical beam size in $e^{+} / e^{-}$storage rings; we differentiate between the two because it is possible to increase the beam size without increasing the emittance. Most of this discussion is taken directly from Ref. 84. A section discussing the synchrotron motion and a section applying the results to the NLC damping ring has been added. The discussion is very general and is applicable to synchrotron radiation sources as well as damping rings; synchrotron radiation sources strive for small emittances to increase the spatial coherence of the photon beams.

We consider contributions from the opening angle of the synchrotron radiation, the vertical dispersion, and the betatron and synchro-betatron couplings; we neglect all current dependent phenomena which also constrain the ring performance, these are discussed in Section 2.3. The goal in performing this study is to illustrate how these effects contribute to the vertical emittance and beam size, and thereby determine the limitations that they impose on future designs. In particular, we will discuss alignment tolerances needed to limit the vertical emittance and beam size that are consistent with the inclusion of realistic correction techniques.

As discussed in Appendix A, the low current equilibrium emittance and beam size in an $e^{+} / e^{-}$storage ring is determined by two competing processes: quantum excitation and damping, both of which result from the synchrotron radiation emitted by the particles in the ring. The quantum excitation is due to the discrete nature of the radiation whereas the damping is a result of the mere existence of the incoherent synchrotron radiation.

The radiation of a photon changes the particle's energy and gives a small transverse kick that depends upon the opening angle of the radiation. The transverse leflections due to the opening angle directly change the amplitude of the particles betatron motion, and thereby the bunch's emittance, while the change in energy due to the radiation has a more subtle effect. The particle executes betatron oscillations about a closed orbit in the ring. Since this closed orbit depends upon the particle energy, the radiation of a photon increases the rms amplitude of the betatron motion 
by displacing the closed orbit relative to the particle's position. In the horizontal plane, the increase in emittance due to this second effect is typically much larger than the increase due to the opening angle of the radiation. However, ideally, in the vertical plane, there is no dispersion and thus the opening angle should determine the vertical emittance.

In practice, this is not the case. First, vertical dipole errors and a non-zero vertical closed orbit in the quadrupole magnets will directly introduce vertical dispersion. Second, a non-zero vertical closed orbit through the sextupole magnets, vertical sextupole misalignments, or rotational misalignments of the quadrupoles couple the horizontal and vertical planes. This coupling has two effects, both of which increase the vertical emittance. It couples the horizontal dispersion to the vertical, increasing the vertical, and it couples the $x$ and $y$ betatron motion so that energy is transferred between the two planes. Finally, misalignments of the RF cavities or vertical dispersion in the RF cavities can couple the transverse and the longitudinal planes.

In this chapter, the effects of the coupling on the vertical emittance are analyzed perturbatively, assuming large aspect ratios $\epsilon_{x} / \epsilon_{y}$ and $\epsilon_{z} / \epsilon_{y}$. In the next section, the relevant equations of motion are introcluced and we discuss the closed orbit and the closed orbit correlation function which will be needed to calculate the effects of a closed orbit. Then, in Section 2.2.3, we calculate the emittance due to the opening angle of the radiation. This is roughly estimated in Ref. 104; the more detailed calculation in Section 2.2.3 y'jelds a result a factor of two smaller.

In Section 2.2.4, we calculate the verlical emittance and beam size due to the vertical dispersion caused by ranclom crrors and a non-zero closed orbit. In previous work, the corrected closed orbit has been treated either as a series of uncorrelated offsets in the magnets ${ }^{[27,82]}$ or the same as an uncorrected closed orbit. ${ }^{[4,112]}$ Typically, the first procedure will overestimate the crfect of the closed orbit and the second will underestimate the contribution.

Next, in Sections 2.2.5 and 2.2.6, we use analogy with the vertical dispersion to discuss the betatron and synchro-betitron coupling. As stated, in an $e^{+} / e^{-}$ring, the betatron and synchro-betatron couplings cause both a local beam size increase and a fundamental dilution of the rerical phase space; the local beam size increase arises because the horizontal and longitudinal emittances are projected into the vertical plane. The betatron compling lass been treated both exactly ${ }^{(26,69,125)}$ and when close to the coupling resonances. ${ }^{[2 c .4 i, 1 x]}$ Likewise, the synchro-betatron coupling has 
been treated exactly ${ }^{[1,19]}$ and when close to resonance. ${ }^{[3,80,113)}$ Unfortunately, the first provides a formalism that is complex and does not lend itself to a simple understanding of the problem, and the second approach is not sufficient in $e^{+} / e^{-}$ rings. In particular, such an analysis of the betatron coupling suggests that the coupling can be fully corrected with a few (2-4) skew quadrupoles. This is not correct; one must fix the coupling at every bending magnet to fully cancel the coupling contribution to the emittance.

In Section 2.2.7, we estimate the effectiveness of various techniques in reducing the vertical emittance and beam size, and we compare these analytic results with the results of simulations. Next, in Section 2.2.8, we discuss the calculation of tolerances, consistent with the correction techniques, to limit the vertical emittance and beam size in future storage ring designs. The results of both Sections 2.2.7 and 2.2.8 are important when determining tolerances to limit the vertical emittance with a specified degree of confidence. Then, in Sections 2.2.9 and 2.2.10, we summarize our results and apply them to calculate tolerances for the NLC damping ring.

Finally, it should be noted that many of the results in Sections 2.2.4 and 2.2.5, in particular, the effect of ranclom errors, have been derived repeatedly over the last thirty years; references to the earlier sources are provided. The new contributions in these sections are: the effects of a corrected closed orbit are calculated more precisely, the distinction between the projected emittance and the emittance is emphasized, and a simple form for the emittance due to betatron coupling is found which is analogous to the emittance due to the vertical dispersion. This later result is important for determining the effectiveness of the coupling correction which is discussed in Section 2.2.7. In addition, the distinction between the beam size and the emittance was obviously' realized by Piwinski in Ref. 82, but it seems to have been neglected in much of the literature. Since this is relevant in damping rings, it is important to emplasize the difference.

\subsubsection{Preliminaries}

A particle beam consists of particles clistributed in six-dimensional phase space. When the beam is uncoupled, the ms vertical emitiance of a matched beam is simply given by:

$$
\epsilon_{y}=\sqrt{\left\langle y^{2}\right\rangle\left\langle y^{\prime 2}\right\rangle-\left\langle y y^{\prime}\right\rangle^{2}}=\frac{\left\langle y^{2}\right\rangle}{\beta_{y}} .
$$

But, when the beam is compled the normal modes of oscillation rotate from the 
horizontal, vertical, and longitudinal planes. In weakly coupled $e^{+} / e^{-}$rings, this coupling has two effects: it increases the projected vertical emittance, the larger horizontal and longitudinal emittances are projected into the vertical phase space, and it couples the "vertical" normal mode emittance to the synchrotron radiation noise, leading to an increase in the normal mode emittance; in a weakly coupled ring, we can discuss the "vertical" normal mode emittance since the normal modes are not rotated far from the uncoupled orientations.

The projected emittance depends upon the coupling and can fluctuate from point-to-point around the ring while the equilibrium normal mode emittance is invariant. Thus, in this chapter, we will refer to the vertical normal mode emittance as the "vertical emittance" and the projected emittance as the local beam size: $\left\langle y^{2}\right\rangle / \beta_{y}$.

We are interested in calculating both the vertical beam size and the "vertical" normal mode emittance; the beam size is the relevant quantity in some situations while the emittance is in others. As mentioned, we need to differentiate between the two because the vertical bean size is the projection of the beam's six-climensional emittance onto the vertical plane. Thus, in addition to depending on the vertical emittance, the vertical beam size is also a function of the local coupling between the vertical plane and the horizontal and longitudinal planes. An example, is the coupling due to vertical dispersion: in this case, the beam size depends on both the vertical emittance and the energy spread, through the vertical dispersion.

In the limit of weak coupling, this relation can be expressed as

$$
\frac{\sigma_{y}^{2}(s)}{\beta_{y}(s)}=\epsilon_{y}+\frac{\sigma_{y \text { local }}^{2}(s)}{\beta_{y}(s)}
$$

where $\sigma_{y}$ local includes the effects of the local coupling and $\epsilon_{y}$ is the "vertica" normal mode emittance. Notice that the local coupling contribution to $\sigma_{y}^{2} / \beta_{y}$ is a function of the azimuthal position in the ring $s$, while the contribution from the emittance is (approximately) invariant. This occurs because the emittance represents a fundamental measure of the vertical phase space while $\sigma_{\text {local }}$ is due to a coupling that can change from point-to-point.

At this point, we can further subdivide the contributions to the vertical beam size. As mentioned in the introduction. we consider four effects that contribute to the low current beam size: (1) the momentum dependance of the vertical closed orbit, 
i.e., the vertical dispersion, (2) the coupling of the betatron motion, (3) the synchrobetatron coupling, and (4) the opening angle of the radiation. These effects have contributions that are statistically independent which is discussed in Appendix C.1. Thus, the vertical beam size can be written as the sum of the contributions:

$$
\frac{\sigma_{y}^{2}(s)}{\beta_{y}(s)}=\frac{\sigma_{\text {dispersion }}^{2}}{\beta_{y}}+\frac{\sigma_{\beta \text { coupling }}^{2}}{\beta_{y}}+\frac{\sigma_{\text {synchro }-\beta \text { coupling }}^{2}}{\beta_{y}}+\epsilon_{\text {opening ang. }} .
$$

Here, the first three contributions have both a local coupling contribution and an emittance contribution while the opening angle only contributes to the vertical emittance.

\subsubsection{EQUATIONS OF MOTION}

To calculate the contributions to the beam size, we will need equations for the vertical dispersion, the betatron motion, and the synchrotron motion. Strictly, the transverse and the lingitudinal motion should be treated together in a complete description of the coupled six-dimensional motion. Fortunately, in a strong-focusing storage ring, the synchrotron frequency is much lower than the betatron frequencies, and thus it is usually valid to assume that the synchrotron motion only causes an adiabatic modulation of the betatron motion; we examine the effects of the synchrotron motion and the synchro-betatron resonances in Section 2.2.6.

The equations of motion for a particle in a storage ring are discussed in Appendix A. Neglecting the effects of synchrotron radiation and acceleration, the transverse equations can be writtcn ${ }^{[100\}}$

$$
\begin{aligned}
x^{\prime \prime}+(1-\Delta)\left[\left(\Lambda_{1}+G^{\prime 2}\right) x+\widetilde{K}_{1} y+\frac{K_{2}^{\prime}}{2}\left(x^{2}-y^{2}\right)\right] & =\Delta G+(1-\Delta) G_{x c} \\
y^{\prime \prime}-(1-\Delta)\left[K_{1} y-\widetilde{K}_{1} x+K_{2} x y\right] & =(1-\Delta) G_{y c},
\end{aligned}
$$

where the primes denote derivatives with respect to $s$, the azimuthal coordinate, and $\Delta$ is the relative energy deviation: $\Delta \equiv\left(p-p_{0}\right) / p$ where $p$ is the particle momentum and $p_{0}$ is the design momentum; to first-order $\Delta$ equals the more common parameter $\delta \equiv\left(p-p_{0}\right) / p_{0}$. In addition, $(i$ is the main horizontal guide field which is the inverse of the local bending radius:

$$
G(s) \equiv \frac{1}{\rho(s)}=\frac{e}{p_{0}} B_{y}
$$

$G_{x c}$ and $G_{y c}$ are the inverse bending radii of additional corrector or error dipole 
fields, and $K_{1}, \widetilde{K}_{1}^{\prime}$, and $K_{2}$ are the normalized quadrupole, skew quadrupole, and sextupole fields:

$$
K_{1}(s) \equiv \frac{e}{p_{0}} \frac{\partial B_{y}}{\partial x} \quad \widetilde{K}_{1}(s) \equiv \frac{e}{p_{0}} \frac{\partial B_{x}}{\partial x} \quad K_{2}^{\prime}(s) \equiv \frac{e}{p_{0}} \frac{\partial^{2} B_{y}}{\partial x^{2}}
$$

With these definitions, positive $G_{x c, y c}$ causes a deflection in the positive $x$ or $y$ direction and positive $K_{1}$ corresponds to focusing in the horizontal plane.

Now, with complete generality, we can separate the solutions into: (1) the onenergy $(\Delta=0)$ inhomogeneous solution, referred to as the closed orbit, (2) the firstorder energy dependence of this closed orbit, referred to as the dispersion function, and (3) the homogeneous solution which is referred to as the betatron motion. Thus, $x=x_{c}+\delta \eta_{x}+x_{\beta}$ where $x_{c}$ is the closed orbit, $\eta_{x}$ is the dispersion function, and $x_{\beta}$ is the betatron motion. Using this expansion in Eq. (2.2.4), we find equations for the closed orbit

$$
\begin{aligned}
x_{c}^{\prime \prime}+\left(\kappa_{1}+G^{2}\right) x_{c}+\tilde{K}_{1} y_{c}+\frac{K_{2}}{2}\left(x_{c}^{2}-y_{c}^{2}\right) & =G_{x c} \\
y_{c}^{\prime \prime}-\kappa_{1} y_{c}+\tilde{K}_{1}^{\prime} x_{c}-K_{2} x_{c} y_{c} & =G_{y c} .
\end{aligned}
$$

Next, linear equations for the dispersion function and the on-energy betatron motion can be found by expanding about the closed orbit:

$$
\begin{aligned}
& \eta_{x}^{\prime \prime}+\left(\Lambda_{1}^{\prime}+G^{2}\right) \eta_{x}+{\widetilde{\Lambda_{1}}}_{1} \eta_{y}+\Lambda_{2}\left(x_{c} \eta_{z}-y_{c} \eta_{y}\right)= \\
& G-G_{x c}+\left(K_{1}+G^{2}\right) x_{c}+\tilde{K}_{1} y_{c}+\frac{K_{2}}{2}\left(x_{c}^{2}-y_{c}^{2}\right) \\
& \eta_{y}^{\prime \prime}-K_{1} \eta_{y}+\tilde{K}_{1}^{\prime} \eta_{x}-\kappa_{2}^{\prime}\left(x_{c} \eta_{y}+y_{c} \eta_{x}\right)= \\
& -G_{y c}-K_{1} y_{c}+\tilde{K}_{1} x_{c}-K_{2} x_{c} y_{c}
\end{aligned}
$$

and

$$
\begin{aligned}
x_{\beta}^{\prime \prime}+\left(K_{1}+G^{2}\right) x_{\beta}+\widetilde{K}_{1 y_{\beta}}+K_{2}\left(x_{c} x_{\beta}-y_{c} y_{\beta}\right) & =0 \\
y_{\beta}^{\prime \prime}-K_{1}^{\prime} y_{\beta}+\widetilde{K}_{1} x_{\beta}-K_{2}\left(x_{\beta} y_{c}+x_{c} y_{\beta}\right) & =0 .
\end{aligned} .
$$

These equations for the transverse motion are complicated. Although, we could solve for the coupled motion and beam sizes exactly, ${ }^{[28,47,89,125]}$ these exact solutions do not provide simple insight into the wealily coupled (flat beam) case. Thus, we will proceed by further approximating these equations of motion. 
Table 3. Effects of rotational $[\Theta]$ and vertical $\left[y_{m}\right]$ misalignments.

\begin{tabular}{|c|c|c|}
\hline Misalignment & Effect & Result \\
\hline iert. BPM & $y_{c} \approx y_{m}$ & non-zero closed orbit \\
\hline Vert. Quad. & $\Delta G_{x}=-K_{1} y_{m}$ & dipole kick \\
\hline Vert. Sext. & $\Delta \widetilde{K}_{1}=K_{2} y_{m}$ & coupling \\
\hline Rot. Bend & $\Delta G_{x}=-G \Theta$ & dipole kick \\
\hline Rot. Quad. & $\Delta \widetilde{K}_{1}=2 K_{1}^{\prime} \Theta$ & coupling \\
\hline
\end{tabular}

\subsubsection{Perturbative Approximation}

In the limit of flat beams, one can solve the equations for the dispersion function and the betation motion perturbatively. For the beam to be flat, the horizontal dispersion must be, on average, much larger than the vertical $\eta_{x} \gg \eta_{y}$ and the horizontal betatron amplitude must be much larger than the vertical $x_{\beta} \gg y_{\beta}$. Furthermore, without a loss in gencrality, we can assume that the horizontal closed orbit is zero; the effect of a non-zero horizontal closed orbit can be included by considering small changes of the focusing function $K_{1}$ due to the sextupoles.

Now, with these approximations, the equations for the dispersion and the betatron motion are

$$
\begin{aligned}
x_{\beta}^{\prime \prime}+\left(K_{1}+G^{2}\right) x_{\beta} & =0 \\
y_{\beta}^{\prime \prime}-K_{1} y_{\beta} & =-\widetilde{K}_{1} x_{\beta}+K_{2}^{\prime} y_{c} x_{\beta},
\end{aligned}
$$

and

$$
\begin{aligned}
\eta_{x}^{\prime \prime}+\left(K_{1}^{\prime}+G^{2}\right) \eta_{x} & =G \\
\eta_{y}^{\prime \prime}-K_{1}^{\prime} \eta_{y} & =-G_{y c}^{\prime}-K_{1}^{\prime} y_{c}-\widetilde{K}_{1}^{\prime \prime} \eta_{x}+K_{2}^{\prime} y_{c} \eta_{x} .
\end{aligned}
$$

These equations are no longer couplecl, the vertical motion is simply driven by the horizontal, and thus they are simple to solve.

\subsubsection{ERrors}

As has been mentioned, excluding the opening angle contribution, the low current vertical beam size is chereminned by errors in an uncoupled storage ring. In this chapter, we consider the offect of random vertical misalignments of the quadrupoles, sextupoles, and the lisam Position Monitors (BPMs). In addition, we will 
also consider the effect of random rotational errors of the quadrupoles and the bending dipoles. The effect of these errors is summarized in Table 3 where $y_{m}$ and $\Theta$ are the vertical and rotational misalignments. As one can see from Eqs. (2.2.10) and (2.2.11), vertical dipole errors, due to rotations of the bends, vertical misalignments of the quadrupoles, or non-zero closed orbits in the quadrupoles directly introduce vertical dispersion. In addition, these same dipole errors create a non-zero vertical closed orbit which couples the $x$ and $y$ planes in the sextupoles. Finally, quadrupole rotations and sextupole misalignments will also couple the two transverse planes. We will discuss these effects in detail in Sections 2.2.4 and 2.2.5 after discussing the opening angle contribution to the emittance.

\subsubsection{Closed Orbit}

Here, we calculate the closed orbit and the closed orbit correlation function resulting from the misalignments. 'The correlation function will be needed, in Sections 2.2.4 and 2.2.5, to calculate the beam sizes resulting from the vertical dispersion and the betatron coupling. Although we are primarily concerned with the effects of corrected orbits, we will derive expressions for both corrected and uncorrected orbits for comparison.

The vertical close orbit is described by Eq. (2.2.7). Assuming that the skew quadrupole terms are small, i.e., the weali coupling limit, Eq. (2.2.7) is easily solved with the periodic Greens function for the ring:

$$
\mathcal{G}_{x, y}\left(s, s^{\prime}\right)=\frac{\sqrt{\beta_{x, y}(s) \beta_{x, y}\left(s^{\prime}\right)}}{2 \sin \pi \nu_{x, y}} \cos \left(\left|\psi \cdot x, y(s)-\psi_{x, y}\left(s^{\prime}\right)\right|-\pi \nu_{x, y}\right),
$$

where $\beta$ is the beta function, $\nu$ is the tune, and $\psi$ is the phase advance: $\psi=\int d s / \beta$. Using this, we find a solution for the vertical closed orbit, ${ }^{(34)}$

$$
y_{c}(s)=\frac{\sqrt{\beta_{y}(s)}}{2 \sin \pi \nu_{y}} \int_{g}^{s+C} \sqrt{\beta_{y}(z)} \cos \left(\psi_{y}(s)-\psi_{y}(z)+\pi \nu_{y}\right) G(z) d z
$$

where $G(s)=G_{y c}+G_{B}^{\prime} \Theta_{B}+K_{1}^{\prime} y_{m}$.

Now, we can calculate the expecterl ims magnitude of the closed orbit given an ensemble of random dipole errors, with Catussian distributions. One finds the woll 
known result ${ }^{[34}$

$$
\left\langle y_{c}^{2}(s)\right\rangle=\frac{\beta_{y}(s)}{8 \sin ^{2} \pi \nu_{y}} \sum_{\text {kicks }}\left\langle G^{2} L^{2}\right\rangle \overline{\beta_{y}},
$$

where the beta function at each kick is approximated by the average beta function within that magnet and the angle brackets are used to denote the expected value which is found by averaging over the gaussian distribution of errors.

Next, to calculate the vertical dispersion and the coupling introduced by the errors, we need the correlation function for the closed orbit, $\left\langle y_{c}\left(s^{\prime}\right) y_{c}(s)\right\rangle$. Using Eq. (2.2.13), this can be expressed as a double integral

$$
\left\langle y_{c}\left(s^{\prime}\right) y_{c}(s)\right\rangle=\frac{\sqrt{\beta(s) \beta\left(s^{\prime}\right)}}{4 \sin ^{2} \pi \nu} \int_{s^{\prime}}^{s^{\prime}+C} d z^{\prime} \int_{s}^{s+C} d z \sqrt{\beta^{\prime} \beta}\left\langle G\left(z^{\prime}\right) G(z) \cos ^{\prime} \cos \right\rangle
$$

where $\beta^{\prime}=\beta\left(z^{\prime}\right), \cos =\cos \left(\iota^{\prime}(s)-\psi^{\prime}(z)+\pi \nu\right)$, and $\cos ^{\prime}=\cos \left(\psi\left(s^{\prime}\right)-\psi\left(z^{\prime}\right)+\pi \nu\right)$. To evaluate $\left\langle G\left(z^{\prime}\right) G(z)\right\rangle$ the integrals must be over the same portion of the ring. Assume initially that $s^{\prime}>s$, then

$$
\left\langle y_{c}\left(s^{\prime}\right) y_{c}(s)\right\rangle=\frac{\sqrt{\beta(s) \beta\left(s^{\prime}\right)}}{4 \sin ^{2} \pi \nu}\left[\int_{s^{\prime}}^{s+C} \int_{s} d z^{\prime} d z \cdots+\int_{s+C}^{s^{\prime}+C} d z^{\prime} \int_{s}^{s^{\prime}} d z \cdots\right]
$$

where $\cdots$ is used to represent the integrands and we have used the fact that the errors $G$ are uncorrelated to simplify the expression.

As before, the double intcgrals collapse to single sums over the deflections, but the second double integral has difforent limits and thus an additional factor of $2 \pi \nu$ must be added to the phase $v^{\prime}\left(z^{\prime}\right)$. We average over the high frequency terms and, in the case of an uncorrected closed orbit, we are left with

$$
\begin{aligned}
\left\langle y_{c}\left(s^{\prime}\right) y_{c}(s)\right\rangle= & \frac{\sqrt{\beta_{y}(s) \beta_{y}\left(s^{\prime}\right)}}{8 \sin ^{2} \pi \nu_{y}}\left[\cos \Delta \psi \sum_{i=s}^{s+C} \beta_{y i}\left\langle G_{i}^{2} L_{i}^{2}\right\rangle\right. \\
& \left.+\left(\cos \left(\left|\Delta \iota^{\prime}\right|-2 \pi \nu_{y}\right)-\cos \Delta \psi\right) \sum_{i=s}^{s^{\prime}} \beta_{y i}\left\langle G_{i}^{2} L_{i}^{2}\right\rangle\right],
\end{aligned}
$$

where $\Delta \psi=\psi_{y}\left(s^{\prime}\right)-\psi_{y}(s)$ and the absolute values signs were used to include the case $s^{\prime}<s$. Note that terms of ordor $1 / 4 \pi \nu_{y}$ have been dropped from Eq. (2.2.17); these will be small corrections in high tune, low emittance rings. 
We consider two cases: an uncorrected orbit and the orbit after substantial correction. Since the correlation function is periodic in $\Delta s$, we can express it as a Fourier series. Furthermore, since the $\sqrt{\beta(s)}$ dependence has been removed, the correlation function must be an even function of $\Delta s$ and thus the Fourier series only contains cosine terms:

$$
\left\langle y_{c}\left(s^{\prime}\right) y_{c}(s)\right\rangle=\sqrt{\beta_{y}(s) \beta_{y}\left(s^{\prime}\right)} \frac{\left\langle y^{2}\right\rangle}{\beta_{y}}\left[\frac{c_{0}}{2}+\sum_{n=1}^{\infty} c_{n} \cos \frac{n \Delta \psi}{\nu_{y}}\right]
$$

To calculate the coefficients $c_{n}$ for an uncorrected orbit, we make a smooth approximation

$$
\left|\sum_{i=s}^{s^{\prime}} \beta_{y i}\left\langle G_{i}^{2} L_{i}^{2}\right\rangle\right| \approx \frac{\left|\Delta \psi^{\prime}\right|}{2 \pi \nu_{y}} \sum_{i=s}^{s+C} \beta_{y i}\left\langle G_{i}^{2} L_{i}^{2}\right\rangle
$$

where $\Delta \psi=\psi\left(s^{\prime}\right)-\psi(s)$. The coefficients are then

$$
c_{n}=\frac{\left(n^{2}+\nu_{y}^{2}\right)\left(1-\cos 2 \pi \nu_{y}\right)}{\pi^{2}\left(n^{2}-\nu_{y}^{2}\right)^{2}} .
$$

Here, only the two harmonics $c_{n}$ on either side of the tune, $n=\left\lfloor\nu_{y}\right\rfloor,\left\lceil\nu_{y}\right\rceil$, will be large, and thus, we can approximate the uncorrected orbit with just these two terms.

When the closed orbit is corrected its Fourier spectrum tends towards that of white noise. There are two reasons for this: first, most orbit correction techniques tend to reduce the dominant harmonics on either side of the tune while increasing the other modes. The second, and more fundamental, reason is that the BPMs are misaligned relative to the ring centcrline. Thus, even with perfect orbit correction, where the measured orbit is zeroed at all of the position monitors, the actual closed orbit will have a white noise spectrum.

We can approximate this by" assuming that the correctors "randomize" the orbit, and thus points on either side of a corrector are uncorrelated. Furthermore, assuming that many correctors are used to correct the orbit, we can approximate the correlation function between correctors with just the first term of $\mathrm{Eq} .(2.2 .17)$. Thus,

$$
\left\langle y_{c}\left(s^{\prime}\right) y_{c}(s)\right\rangle_{\text {cor }}=\sqrt{\beta_{y}(s) \beta_{y}\left(s^{\prime}\right) \frac{\left\langle y_{c}^{2}\right\rangle}{\beta_{y}}} \begin{cases}\cos \Delta \psi, & \text { No correctors } \\ \text { between } s \text { and } s^{\prime} \\ 0, & \text { Otherwise }\end{cases}
$$

Here, the term $\left\langle y_{c}^{2}\right\rangle / \beta_{y}$ is not equall to Iicl. (2.2.14); it is the square of the residual 
orbit after correction. In particular, for an orbit that is fully corrected, one can approximate $\left\langle y_{c}^{2}\right\rangle$ with the estimated vertical misalignments of the BPMs $\left\langle y_{m}^{2}\right\rangle$. We will use this correlation function in Sections 2.2 .4 and 2.2 .5 after calculating the effect of the opening angle of the radiation.

\subsubsection{Opening Angle Emittance}

In this section, we derive the emittance contribution due to the opening angle of the synchrotron radiation. Photons are radiated with an rms angle of $1 / \gamma$ relative to the particle trajectory, thereby changing both the longitudinal and transverse momentum of the particle. In an icleal storage ring built in the horizontal plane, the vertical closed orbit does not depend upon the longitudinal momentum and thus the radiation opening angle should determine the vertical emittance. In practice, errors in the machine will generate vertical dispersion and couple the horizontal and vertical betatron motion. These effects will then determine the vertical emittance. Still, the emittance due to the opening angle is useful since it specifies a lower bound on the vertical emittance, a lower bound that will be approached by future machines.

The emittance contribution due to the opening angle is estimated in Ref. 104 by ignoring the correlation between the energy and angle of the radiated photons. In this approximation, one finds

$$
c_{y} \simeq \frac{C_{\eta}}{2 J_{y}} \frac{\oint \beta_{y}(s)\left|G^{3}(s)\right| d s}{\oint G^{2}(s) d s}
$$

where $C_{q}=3.84 \times 10^{-13}$ meters. Our derivation will parallel that of Ref. 104 , except that the correlation between the energy and angle of the photon will be included. The high energy photons should be radiated at smaller angles than the low energy photons and thus the correct, result, will be smaller than Eq. (2.2.22).

When a particle radiatcs a plioton of energy $u$, the transverse angle changes

$$
\Delta y^{\prime}=\frac{u}{E_{0}} \Theta_{y} \quad \text { and } \quad \Delta y=0
$$

where $\Theta_{y}$ is the angle of inclination between the particle trajectory and the bath of the photon. The change in $y^{\prime}$ changes the particle's transverse invariant $J_{y}$ :

$$
\Delta J_{y}=\left(\alpha_{y} y \Delta y^{\prime}+\beta_{y} y^{\prime} \Delta y^{\prime}+\frac{\beta_{y}}{2}\left(\Delta y^{\prime}\right)^{2}\right),
$$

where $J_{y}$ is defined in Eq. (A.2.21). 
Now, the beam emittance is calculated from $J_{y}$ by averaging over the beam. Since changes in $y^{\prime}$ are statistically independent, the change in the emittance is found from the average of $\Delta J_{y}$. Furthermore, the averages of $y$ and $y^{\prime}$ are zero, and thus, if we assume that the probability of radiation is uncorrelated with the particle position and transverse momentum, the change in the emittance between position $s$ and $s+d s$ is

$$
d \epsilon(s)=\frac{\beta_{y}}{2} \mathcal{N}\left\langle\Delta y^{\prime 2}(s)\right\rangle \frac{d s}{c}
$$

where the angle brackets are used to denote an average over the particles in the beam and

$$
\mathcal{N}\left\langle\Delta y^{\prime 2}(s)\right\rangle=\int \frac{u^{2} \Theta_{y}^{2}}{E_{1}^{2}} n(u, \Omega, s) d u d \Omega
$$

Here, $n(u, \Omega, s) d u d \Omega$ is the expected number of photons radiated per unit time at position $s$ with an energy between $u$ and $u+d u$ and a solid angle of $\Omega$ to $\Omega+d \Omega$.

By assuming that $\left\langle y \Delta y^{\prime}\right\rangle=\langle y\rangle\left\langle\Delta y^{\prime}\right\rangle=0$, as we did in Eq. (2.2.25), we are ignoring the effect of gradients in the magnets. When the magnetic field has a gradient, the probability of radiation depends upon the particle position. But, the magnetic field variation across a beam is typically very small and thus we can ignore it. For example, a damping ring design for the NLC ${ }^{(00)}$ has gradients of 300 $\mathrm{KG} /$ meter in the $13.1 \mathrm{KG}$ bending magnets while the beam sigma is 4 microns. Thus, the field varies by only' 2 Gauss across the beam.

Now, to find the change in the emittance over one turn, we integrate $d \epsilon$ over the ring

$$
\Delta \epsilon=\oint \frac{\beta_{y}}{2} N\left(\Delta y^{\prime 2}\right\rangle \frac{d s}{c}
$$

The equilibrium emittance is then calculated by setting the change due to quantum excitation equal to the change due to clamping. Thus

$$
\epsilon_{y}=\frac{T_{y}}{4 T_{0}} \oint \frac{d s}{c} \beta_{y}(s) \int \frac{u^{2} \Theta_{y}^{2}}{E_{0}^{2}} n(u, \Omega, s) d u d \Omega
$$

where $\tau_{y}$ is the vertical clamping time and $T_{0}$ is the revolution period of the ring. Note that the vertical emittance clamping rate is $2 / r_{y}$. 
Thus, we need to evaluate the integral in Eq. (2.2.28) over $u$ and $\Omega$. The rate of photons emitted with energy' between $u$ and $u+d u$ multiplied by the energy $u$ is equal to the power radiated with a frequency between $\omega=u / \hbar$ and $(u+d u) / \hbar$.

$$
u n(u, \Omega, s) d u d \Omega=\frac{\partial^{2} P(u / \hbar, \Omega, s)}{\partial \omega \partial \Omega} d \omega d \Omega
$$

The classical relation for the differential power radiated by an ultra-relativistic electron in instantaneous circular motion was calculated by Schwinger ${ }^{[200]}$

$$
\frac{d^{2} P(\omega, \psi, s)}{d \omega d \psi}=\frac{e^{2}}{3 \pi^{2} c^{2}} \rho(s) \omega^{2}\left(\frac{1}{\gamma^{2}}+\psi^{2}\right)^{2}\left[K_{\frac{2}{3}}^{2}(\xi)+\frac{\psi^{2}}{1 / \gamma^{2}+\psi^{2}} K_{\frac{1}{3}}^{2}(\xi)\right]
$$

where

$$
\xi \equiv \frac{\omega \rho}{3 c}\left(\frac{1}{\gamma^{2}}+\psi^{2}\right)^{\frac{3}{2}}
$$

Here, $\psi$ is the angle of inclination above the orbital plane; thus, $\psi$ is equivalent to $\Theta_{y}$ of $\mathrm{Eq}$. (2.2.23). In addition, $\rho(s)$ is the instantaneous radius of curvature, and $K_{\frac{1}{3}}$ and $K_{\frac{2}{3}}$ are modified Bessel functions. Notice that the azimuthal angle has been integrated out of $\mathrm{Eq} .(2.2 .30)$; it would be needed if we wanted to calculate the opening angle contribution to the horizontal emittance, but, as was mentioned earlier, the horizontal emittance is clominated by the dispersive effects.

Thus, the equilibrium emititance is

$$
\begin{aligned}
\epsilon_{y}=\frac{\tau_{y}}{4 T_{0}} \frac{27}{\pi^{2}} \frac{c e^{2} h}{E_{0}^{2}} \oint d s & \left.\frac{\beta_{y}}{\left|\rho^{3}\right|} \int_{-\frac{\pi}{2}}^{\frac{\pi}{2}} d \psi \psi^{2}\left(\frac{1}{\gamma^{2}}+\psi\right)^{2}\right)^{-4} \\
& \times \int_{0}^{\infty} d \xi \xi^{3}\left[K_{\frac{2}{3}}^{2}(\xi)+\frac{\psi^{2}}{1 / \gamma^{2}+\psi^{2}} K_{\frac{1}{3}}^{2}(\xi)\right]
\end{aligned}
$$

where $\omega$ has been written in terms of $\xi$. Furthermore, since the integrand is very small for $\psi \sim \pi / 2 \gg 1 / \gamma$, and decreases rapidly with $\psi$, we can extend the limits of integration from $\pm \pi / 2$ to $\pm \infty$

$$
\epsilon_{y}=\frac{\tau_{y}}{4 T_{0}} \frac{27}{\pi^{2}} \frac{c \epsilon^{2} \hbar}{E_{0}^{2}} \oint d s \frac{\beta_{y}}{\left|r^{3}\right|} \int_{-\infty}^{\infty} d \psi^{\prime} \psi^{2}\left(\frac{1}{\gamma^{2}}+\psi^{2}\right)^{-4}\left[I_{3}\left(\frac{2}{3}\right)+\frac{\psi^{2}}{1 / \gamma^{2}+\psi^{2}} I_{3}\left(\frac{1}{3}\right)\right]
$$


where

$$
I_{n}(\nu) \equiv \int_{0}^{\infty} d \xi \xi^{n} K_{\nu}^{2}(\xi)
$$

The integral $I_{n}(\nu)$ is equal to ${ }^{|44|}$

$$
I_{n}(\nu)=\frac{1}{4} \frac{\Gamma\left(\frac{1}{2}\right) \Gamma\left(\frac{n+1}{2}\right)}{\Gamma\left(\frac{n}{2}+1\right)} \Gamma\left(\frac{n+1}{2}+\nu\right) \Gamma\left(\frac{n+1}{2}-\nu\right)
$$

where $\Gamma(x)$ is the gamma function. Specifically,

$$
I_{3}(\nu)=\frac{1}{3} \frac{\nu \pi}{\sin \pi \nu}\left(1-\nu^{2}\right)
$$

Next, the integral over $\psi$ is perlormed using the algebraic integral $\left.\right|^{\mid 4 s]}$

$$
\int_{0}^{\infty} \frac{x^{2 m} d x}{\left(x^{2}+c\right)^{n}}=\frac{(2 m-1) ! !(2 n-2 m-3) ! ! \pi}{2(2 n-2) ! ! c^{n-m-1} \sqrt{c}}
$$

Finally, substituting for $\tau_{y}$, ${ }^{(101)}$ we find for the opening angle contribution to the emittance,

$$
\epsilon_{y}=\frac{13}{55} \frac{C_{\eta}}{\mathcal{J}_{y}} \frac{\oint \beta_{y}(s)\left|G^{3}(s)\right| d s}{\oint G^{2}(s) d s} .
$$

This is a factor of 2.1 times smaller than the estimate in Eq. (2.2.22). This expression can be further simplified by using the average value of $\beta_{y}$ and the rms energy spread (Eq. $(A, 5.6))$. We find

$$
\gamma \epsilon_{y} \approx 0.24 \mathcal{J}_{\varepsilon} \overline{\beta_{y}} \frac{\sigma_{\varepsilon}^{2}}{\gamma}
$$

where $\gamma \epsilon$ is the normalized emittance and $\mathcal{J}_{\epsilon}$ and $\sigma_{\epsilon}$ are the longitudinal damping partition number and the rms energy spread.

To estimate the importance of this effect, we note that opening angle emittance is roughly a factor of $10^{5}$ smaller than the SLC damping ring design emittance, a factor of $10^{3}$ smaller than the Aclvanced Light. Source ${ }^{[129]}$ (ALS) design vertical emittance $\left(\epsilon_{x} / \epsilon_{y}=10\right)$, and a factor of dis smaller than the NLC damping ring design vertical emittance. 


\subsubsection{Vertical Dispersion}

The vertical dispersion increases the beam size in two ways: first, the vertical dispersion is a coupling between the longitudinal energy deviation and the vertical position. Since the beam contains a finite energy spread, the vertical dispersion directly contributes to the vertical beam size. This will be referred to as the "local" contribution since the beam size increase only depends upon the local value of the vertical dispersion; the energy spread in the beam does not vary significantly around the ring.

Second, the vertical dispersion couples the "noise" due to the synchrotron radiation from the longitudinal plane to the vertical plane. Since the photons are uncorrelated, the radiation causes an increase in the rms amplitude of the betatron oscillation. This effect will be referred to as the "global" contribution of the vertical dispersion since the effect depends upon the value of the dispersion in all of the bending magnets.

Although, both the local and the global contributions from the vertical dispersion increase the vertical beam size, there is a fundamental difference between the two. The local effect is simply clue to a coupling between the longitudinal and vertical planes; it does not actually' cliange the beam's six-dimensional emittance. In contrast, the global effect of the dispersion does cause an increase in the beam emittance. In a synchrotron light source, the distinction between the local and global effects is irrelevant; one is only interested in projected beam size. But, in a damping ring, the distinction is important since one is interested in the extracted beam emittance; in theory, any residual coupling can be removed.

To calculate these two effects, we will first clerive expressions for the dispersion arising from random errors and a non-zero closed orbit. Then, we will calculate the contributions to the vertical bean size and the emittance. Finally, the calculations will be compared with the results of simulations.

\subsubsection{Vertical Dispersion}

To find the vertical beam size contribution due to dispersion, we need to first solve for the vertical dispersion and the derivative. In the limit of flat beams, the vertical dispersion is given by:

$$
\begin{aligned}
\eta_{x}^{\prime \prime}+\left(K_{1}+G^{2}\right) \eta_{x x} & =C_{i} \\
\eta_{y}^{\prime \prime}-K_{1}^{\prime} \eta_{y y} & =-G_{y c}-K_{1}^{\prime} y_{c}-\widetilde{K}_{1} \eta_{x}+K_{2}^{\prime} y_{c} \eta_{x} .
\end{aligned}
$$


These equations are solved in the same manner as the equation for the closed orbit, namely, by using the periodic Grecus function for the focusing field of the ring, Eq. (2.2.12). The solutions are ${ }^{(34)}$

$$
\begin{aligned}
& \eta_{x}(s)=\frac{\sqrt{\beta_{x}(s)}}{2 \sin \pi \nu_{x}} \int_{s}^{s+C} \sqrt{\beta_{x}(z)} \cos \left(\psi_{x}(s)-\psi_{x}(z)+\pi \nu_{x}\right) G(z) d z \\
& \eta_{y}(s)=\frac{\sqrt{\beta_{y}(s)}}{2 \sin \pi \nu_{y}} \int_{s}^{s+C} \sqrt{\beta_{y}(z)} \cos \left(\psi_{y}(s)-\psi_{y}(z)+\pi \nu_{y}\right) F(z) d z
\end{aligned}
$$

where $G(s)$ is the main bending function and $F(s)=\left(K_{2} \eta_{x}-K_{1}\right) y_{c}-\widetilde{K}_{1} \eta_{x}-G_{y c}$.

Now, the derivative of $\eta_{y}$ with respect to $s$ can be found directly from Eq. (2.2.40). Unfortunately, this is complicaled by the beta function which is also a function of $s$. Instead, the function $\eta_{y}^{*}$, which is a function of $\eta_{y}$ and its derivative, is introduced: ${ }^{[82\}}$

$$
\eta_{y}^{*}(s) \equiv \alpha_{y} \eta_{y}+\beta_{y} \eta_{y}^{\prime}
$$

This function is convenient since it both simplifies the expression for the vertical emittance and has a solution that can be expressed in a form similar to (2.2.40):

$$
\eta_{y}^{*}(s)=-\frac{\sqrt{\beta_{y}(s)}}{2 \sin \pi \nu_{y}} \int_{s}^{s+C} \sqrt{\beta_{y}(z)} \sin \left(\psi_{y}^{\prime}(s)-\psi_{y}(z)+\pi \nu_{y}\right) F(z) d z
$$

Because the two equations have similar forms, the calculation of $\eta_{y}^{* 2}$ will parallel that of $\eta_{y}^{2}$. In particular, we will sce that for random errors the expected values of $\eta_{y}^{2}$ and $\eta_{y}^{* 2}$ are equal.

\subsubsection{RANDOM ERRORS}

To estimate the beam size contribution. we need to calculate the expected values of $\eta_{y}^{2} / \beta_{y}$ and $\eta_{y}^{* 2} / \beta_{y}$ for the various error distributions. The square of the vertical dispersion, Eq. (2.2.40), is a doublo integlal

$$
\frac{\left\langle\eta_{y}^{2}\right\rangle}{\beta_{y}}=\frac{1}{4 \sin ^{2} \pi \nu_{y}} \int_{s}^{s+C^{\prime}} \int d z d z^{\prime} \sqrt{\beta \beta^{\prime}}\left\langle\cos \cos ^{\prime} F^{2}\left(z, z^{\prime}\right)\right\rangle
$$

where $\beta^{\prime}, \cos$, and $\cos ^{\prime}$ are defined as they were in Eq. (2.2.15). In addition, since the errors considered (quadrupole rol ations, sextupole misalignments, and dipole errors) 
are all assumed to be statistically independent, the function $F^{2}\left(z, z^{\prime}\right)$ contains five terms

$$
\begin{aligned}
\left\langle F^{2}\left(z, z^{\prime}\right)\right\rangle & =4 K_{1}^{\prime} \eta_{x} I_{1}^{\prime} \eta_{x}^{\prime}\left\langle\Theta \Theta^{\prime}\right\rangle+K_{2}^{\prime} \eta_{x} K_{2}^{\prime} \eta_{x}^{\prime}\left\langle y_{m} y_{m}^{\prime}\right\rangle \\
& +\left\langle G_{y} G_{y}^{\prime}\right\rangle-2 f^{\prime}\left\langle G_{y} y_{c}^{\prime}\right\rangle+f f^{\prime}\left\langle y_{c} y_{c}^{\prime}\right\rangle,
\end{aligned}
$$

where the primes are used to denote functions of $z^{\prime}$ instead of $z$ and $f(z)$ is proportional to the local chromaticity, $f(z)=K_{2} \eta_{x}-K_{1}$; the chromaticity is given by $^{[34]}$

$$
\xi_{y} \equiv \frac{d \nu_{y}}{d p / p_{0}}=\frac{1}{4 \pi} \oint\left(K_{1}-K_{2} \eta_{x}\right) \beta_{y} d s \quad,
$$

where we have neglected the term from combined function magnets. It is important to notice that the vertical dispersion due to a closed orbit can be reduced by using local chromatic correction which reduces $f(z)$; this will be discussed further subsequent!y.

Since the errors are uncorrelated, the first three terms of Eq. (2.2.43) are calculated in the same manner as $\left\langle y_{c}^{2}\right\rangle / \beta_{y}, \mathrm{Eq} .(2.2 .14)$, yielding the results ${ }^{[34]}$

$$
\begin{aligned}
\frac{\left\langle\eta_{y}^{2}\right\rangle_{\text {quad rotation }}}{\beta_{y}} & =\frac{1}{\delta \sin ^{2} \pi \nu_{y}} \sum_{\text {quad }}\left(K_{1} L\right)^{2} 4\left\langle\Theta^{2}\right\rangle \beta_{y} \eta_{x}^{2} \\
\frac{\left\langle\eta_{y}^{2}\right\rangle_{\text {sext misalign }}}{\beta_{y}} & =\frac{1}{\delta \sin ^{2} \pi \nu_{y}} \sum_{\text {sext }}\left(K_{2} L\right)^{2}\left\langle y_{m}^{2}\right\rangle \beta_{y} \eta_{x}^{2} \\
\frac{\left\langle\eta_{y}^{2}\right\rangle_{\text {dipole kicks }}}{\beta_{y}} & =\frac{\left\langle y_{c}^{2}\right\rangle}{\beta_{y}} .
\end{aligned}
$$

All of the above equations are applicable for both corrected or uncorrected orbits. The first two do not depend upon the closed orbit and the term $\left\langle y_{c}^{2}\right\rangle / \beta_{y}$ in the third equation is equal to Eq. (2.2.14) for an uncorrected orbit or the square of the residual for a corrected orbit. Notice that this third term is not a result of a non-zero closed orbit; it results from the errors and correctors that create the non-zero closed orbit.

Finally, note that we have calculated the expected value of $\left\langle\eta_{y}^{2}\right\rangle / \beta_{y}$, but to calculate the emittance wo will also need to calculate $\left\langle\eta_{y}^{* 2}\right\rangle / \beta_{y}$. As mentioned, this quantity is calculated in an analogous manner; it differs from $\left\langle\eta_{y}^{2}\right\rangle / \beta_{y}$ in that $\cos$ and $\cos ^{\prime}$ become sin and sin', but with the same approximations, the results are identical. 


\subsubsection{ORBIT ERRORS}

The fourth and fifth terms of $E_{q} \cdot(2.2 .43)$ are functions of the closed orbit. We will treat each of these terms in turn. First, we express the fourth term as an integral of the closed orbit correlation function:

$$
\left(\frac{\left\langle\eta_{y}^{2}\right\rangle}{\beta_{y}}\right)_{4}=\frac{-2}{2 \sqrt{\beta(s)} \sin \pi \nu} \int_{s}^{s+C} f^{\prime} \sqrt{\beta_{y}^{\prime}\left(z^{\prime}\right)} \cos ^{\prime}\left\langle y_{c}(s) y_{c}\left(z^{\prime}\right)\right\rangle d z^{\prime}
$$

where the subscript 4 is used to denote that this is the fourth term of Eq. (2.2.44).

Next, using the Fourier expansion for the closed orbit function, Eq. (2.2.18), we calculate this integral. In addition, since we are not interested in the explicit dependence on position, we can average over $s$. This yields

$$
\overline{\left(\frac{\left\langle\eta_{y}^{2}\right\rangle}{\beta_{y}}\right)_{4}}=2 \xi_{y} \frac{\left\langle y_{c}^{2}\right\rangle}{\beta_{y}} \sum_{n, \pm} \frac{c_{n}}{\nu_{y} \pm n},
$$

where $\xi_{y}$ is the chromaticity defined in Eq. (A.2.17) and the \pm is used to represent a sum over both $1 /\left(\nu_{y}+n\right)$ and $1 /\left(\nu_{y}-n\right)$. Now, if we only keep the most significant coefficients $c_{n}$ for an uncorrected orbit, this becomes

$$
\overline{\left(\frac{\left\langle\eta_{y}^{2}\right\rangle}{\beta_{y}}\right)_{4}} \approx \frac{2 \pi \xi_{y}}{\sin \pi \nu_{y}} \frac{\left\langle y_{c}^{2}\right\rangle}{\beta_{y}} \operatorname{sinc}^{3} \pi \Delta \nu_{y}
$$

where $\Delta \nu_{y}$ is the fractional part of the tune and $\operatorname{sinc} x=\sin x / x$.

We can calculate this term for a corrected orbit in a similar manner. This yields

$$
\overline{\left(\frac{\left\langle\eta_{y}^{2}\right\rangle}{\beta_{y}}\right)_{4}} \approx \frac{2 \pi \xi_{y}}{\sin \pi \nu_{y}} \frac{\left\langle y_{c}^{2}\right\rangle}{\beta_{y}} \frac{\cos \pi \nu_{y}}{N_{\text {cor }}} .
$$

Here, $N_{\text {cor }}$ is equal to the number of correctors which we have assumed are uniformly distributed. Notice that, although the corrected result is smaller by $1 / N_{\text {corr }}$, the forms of the corrected and uncorrcted results are similar. In particular, note that both results depend linearly upon the chromaticity $\xi_{y}$, which is usually adjusted to be near zero; thus, both Eqs. (2.2.49) and (2.2.50) will tend to be small. 
Now, we turn to the last term in Eq. (2.2.43). This contains the closed orbit correlation function, Eq. (2.2.17). To solve for the effect of an uncorrected orbit, we again use the Fourier expansion for the correlation function. This yields

$$
\left(\frac{\left\langle\eta_{y}^{2}\right\rangle}{\beta_{y}}\right)_{5}=\frac{1}{4 \sin ^{2} \pi \nu_{y}} \frac{\left\langle y_{c}^{2}\right\rangle}{\beta_{y}} \int_{g}^{s+C^{\prime}} \int^{\prime} d z d z^{\prime} f f^{\prime} \beta_{y} \beta_{y}^{\prime} \cos \cos ^{\prime} \sum_{n} c_{n} \cos \frac{n \Delta \psi}{\nu_{y}}
$$

where $\Delta \psi=\psi(z)-\psi\left(z^{\prime}\right)$. This expression is evaluated in the same manner as the previous case; we first calculate the integrals and then average over $s$. We find

$$
\begin{aligned}
\overline{\left(\frac{\left\langle\eta_{y}^{2}\right\rangle}{\beta_{y}}\right)_{5}}=\frac{1}{16 \sin ^{2} \pi \nu_{y}} \frac{\left\langle y_{c}^{2}\right\rangle}{\beta_{y}} \sum_{n=0}^{\infty} c_{n} & {\left[\left|\int_{0}^{C} d z f \beta_{y} e^{i \psi\left(1-n / \nu_{y}\right)}\right|^{2}\right.} \\
& \left.+\left|\int_{0}^{C} d z f \beta_{y} e^{i \psi\left(1+n / \nu_{y}\right)}\right|^{2}\right] .
\end{aligned}
$$

We can approximate this by assuming that the fractional tune $\Delta \nu_{y}$ is small and thus we keep only the most significant coefficient $c_{n}$. This yields

$$
\overline{\left(\frac{\left\langle\eta_{y}^{2}\right\rangle}{\beta_{y}}\right)_{5}} \approx \frac{1}{16 \sin ^{2} \pi \nu_{y}} \frac{\left\langle y_{c}^{2}\right\rangle}{\beta_{y}} \operatorname{sinc}^{4} \pi \Delta \nu_{y}\left(\left(4 \pi \xi_{y}\right)^{2}+(4 \pi \Delta)^{2}\right)
$$

where $\Delta$ is

$$
\Delta=\frac{1}{4 \pi}\left|\int_{0}^{C} d z f \beta_{y} e^{i 2 \psi^{\prime}}\right|
$$

and is sometimes referred to as the width of the off-energy stop band. This result is similar to the result found in Ref. (it; the primary difference is in the appearance of the coefficients although they actually have the same value over much of the range in tune.

Finally, we solve for the effret of a corrected orbit in a similar manner, finding

$$
\overline{\left(\frac{\left\langle\eta_{y}^{2}\right\rangle}{\beta_{y}}\right)_{5}} \approx \frac{1}{16 \sin ^{2} \pi \nu_{y}} \frac{\left\langle y_{c}^{2}\right\rangle}{\beta_{y}} \sum_{n_{r}}^{N_{\text {cor }}}\left[\left|\int_{n_{c}}^{n_{c}+1} d z f \beta_{y}\right|^{2}+\left|\int_{n_{c}}^{n_{c}+1} d z f \beta_{y} e^{i 2 \psi}\right|^{2}\right]
$$

where $n_{c}$ denotes the position of one corrector and $n_{c}+1$ denotes the position of the next corrector. This result differs significantly from the uncorrected case. The 
uncorrected case depends on the average values of $f \beta_{y}$ and $f \beta_{y} e^{i 2 \psi}$. In general, these will be small; the former, the chromaticity, is usually small by design while the later tends to be small because of the oscillating term $e^{i 2 \psi}$. In contrast, the corrected case depends on what we refer to as the local chromaticity and the local $\Delta$. Both of these will typically be much larger than the average values. The local chromaticity is usually positive in regions of dispersion to compensate the negative values in the dispersion free regions. While the average may be zero, the local values are not. In addition, the local value of $\Delta$ will tend to be much larger than the average since the oscillating term does not vary significantly over a short region.

Of course, despite the larger values of $\xi_{\text {local }}$ and $\Delta_{\text {local }}$, the dispersion of the corrected orbit will usually be smaller than that of an uncorrected orbit; orbit correction reduces the residual orljit $\left\langle y_{c}^{2}\right\rangle / \beta$. Furthermore, if the closed orbit is comparable in magnitude to the misalignments $y_{c} \approx y_{m}$, the contribution to the vertical dispersion from the closed orbit will usually be much less than that from the misalignments. This occurs because the orbit, even after correction, is still correlated for short segments and some of the quadrupole and sextupole deflections cancel.

\subsubsection{BEAM SIZE}

At this point, we can solve for the beam size increase due to the vertical dispersion. As mentioned, the vertical clispersion has two effects: (1) it directly increases the beam size by coupling a particle's energy deviation to the vertical position, and (2) it causes the vertical emittance to increase. The first effect is simple; it causes a local contribution to the expected beam size of

$$
\frac{\left\langle\sigma_{y}^{2}(s)\right\rangle_{\text {local }}}{\beta_{y}(s)}=\frac{\left\langle\eta_{y}^{2}(s)\right\rangle}{\beta_{y}(s)} \sigma_{\epsilon}^{2}
$$

where $\sigma_{\epsilon}$ is the rms energy deviation in the beam.

To calculate the second effect, the enittance increase, we use the dispersion invariant $\mathcal{H}_{y}$ which is analogous to $\mathcal{H}_{x^{\prime}}$, lefined in Eq. (A.5.9). Thus, $\mathcal{H}_{y}$ can be expressed

$$
\mathcal{H}_{y}(s)=\frac{1}{\beta_{y}}\left(\eta_{y}^{2}+\eta_{y}^{* 2}\right)
$$

where we have used the function $\eta_{y}^{*}$ introduced in Eq. (2.2.41). The contribution to 
the emittance from the vertical dispersion is ${ }^{(104)}$

$$
\epsilon_{y}=\frac{C_{q} \gamma^{2}}{\mathcal{J}_{y}} \frac{\oint\left|G^{3}(s)\right| \mathcal{H}_{y}(s) d s}{\oint G^{2}(s) d s}
$$

where $C_{q}=55 \hbar /(32 \sqrt{3} m c)=3.84 \times 10^{-13}$ meter and $\mathcal{J}_{y}$ is the vertical damping partition. For a ring in the horizontal plane $\mathcal{J}_{y}=1$; in the limit of weak coupling, the change in $\mathcal{J}_{y}$ due to error's is negligible.

Since the expected values of $\left\langle\eta_{y}^{2}\right\rangle / \beta_{y}$ and $\left\langle\eta_{y}^{* 2}\right\rangle / \beta_{y}$ are equal and are independent of $s$, the expected value of the emittance can be written

$$
\left\langle\epsilon_{y}\right\rangle=2 \frac{C_{y} \gamma^{2}}{\mathcal{J}_{y}} \frac{\left\langle\eta \eta_{y}^{2}\right\rangle}{\beta_{y}} \frac{\oint|G|^{3} d s}{\oint G^{2} d s}=2 \mathcal{J}_{\epsilon} \frac{\left\langle\eta_{y}^{2}\right\rangle}{\beta_{y}} \sigma_{\epsilon}^{2}
$$

where, the relative energy' spreacl (Eq. (A.5.6)) has been used to simplify the expression and $\mathcal{J}_{\epsilon}$ is the longitudinal damping partition. Since $\mathcal{J}_{\epsilon}$ is typically between 1 and 2 , one can see that the cmittance generally has a larger contribution to the beam size than the coupling increase of $\mathrm{Eq}$. (2.2.56).

At this point, we will again emphasize the distinction between these two effects. As mentioned, the first effect, Eq. (2.2.56), is due to a coupling between the energy deviation and the vertical position; it does not change the bearn's six-dimensional emittance. In contrast, the second effect, Eq. (2.2.58), causes a fundamental increase in the phase space volume occupied by the beam. In a synchrotron light source this distinction is irrelevant, but in a damping ring it is important because, unlike the first effect, the emittance increase cannot be corrected once the beam has been extracted from the ring. Of course, both effects can be corrected by correcting the vertical dispersion in the ring; this is the subject of Section 2.2.7.

\subsubsection{Simulations}

To verify these results, the computer program CEMIT ${ }^{[80]}$ has been used to simulate various errors in the SILC! North Damping Ring (NDR); ${ }^{[122,128]}$ the CEMIT program calculates the closed orbit and dispersion and then finds the equilibrium emittance by calculating gencralized synchrotron integrals. The NDR is designed to operate on the coupling difference resonance, but for these simulations the tunes were shifted to $\nu_{x}=8.375$ and $\nu_{y}=3.275$; this lattice will be referred to as the 
Table 4. $\epsilon_{y}$ from vertical dispersion due to misalignments in the NDR1.

\begin{tabular}{|c|c|c|}
\hline Misalignment & Calc, $\epsilon_{y}[\mathrm{~m}$-rad $]$ & Simulated $\epsilon_{y}[\mathrm{~m}$-rad $]$ \\
\hline Random quad. $\Theta=0.5 \mathrm{mrad}$ & $1.91 \times 10^{-12}$ & $1.83 \pm 0.05 \times 10^{-12}$ \\
\hline Random sext. $y_{m}=150 \mu \mathrm{m}$ & $6.51 \times 10^{-12}$ & $6.42 \pm 0.16 \times 10^{-12}$ \\
\hline $\begin{array}{c}\text { Corrected closed orbit due to random } \\
\text { quad. } y_{m}=150 \mu \mathrm{m} \text { and } \mathrm{BPM} y_{m}=150 \mu \mathrm{m}\end{array}$ & $1.32 \times 10^{-12}$ & $1.1 \pm 0.2 \times 10^{-12}$ \\
\hline
\end{tabular}

NDR1. Finally, in this comparison, we will only discuss the increase in the vertical emittance due to the vertical clispersion.

The results of simulating rotational misalignments in the quadrupoles and vertical misalignments in the sextupoles are listed in Table 4 . The misalignments were generated from gaussian distributions with l'ms widths of $0.5 \mathrm{mrad}$ and $150 \mu \mathrm{m}$, respectively. The calculated values are found using Eqs. (2.2.45) and (2.2.59), while the simulated values are found by averaging the results from 1000 different random error distributions. Finally, the simulated error's are the standard errors of the averaged values. In both cases, one can see that the approximate formula agree well with the simulations.

In addition, Table 4 lists results from simulating the effects of a corrected closed orbit. Here, the results are the average of twenty simulations. The simulations included vertical quadrupole misaligmments with an rms of $150 \mu \mathrm{m}$ and vertical BPM misalignments, also, with an rms of $150 \mu \mathrm{m}$. The resulting orbit was corrected using the twenty vertical dipole correctors in the NDR to minimize the rms of the simulated orbit. Before correction, the rms magnitucle of the actual orbit was roughly $1.5 \mathrm{~mm}$; the correction reduced this to $140 \mu \mathrm{m}$, roughly the accuracy of the BPM alignment. In this case, the calculated result was found from Eqs. (2.2.46), (2.2.50), (2.2.55), and (2.2.59), although the dominant contribution comes from Eq. (2.2.55). Again, the calculated estimate agrees well with the average of the simulations. Finally, notice that the contribution from the corrected orbit is less than the contribution due to similar misalignments in the sextupoles; as mentioned, this occurs since the orbit is still correlated over short segments.

To further study the effect of a corrected closed orbit, the average $\overline{\left\langle\eta_{y}^{2}\right\rangle} / \beta_{y}$ has been plotted versus the chromaticity of the ring. In Figure 2, the closed orbit was not corrected while in Figure 3 the orbit was corrected with twenty correctors. Again, the data and errors were found from the results of twenty simulations. The lines 


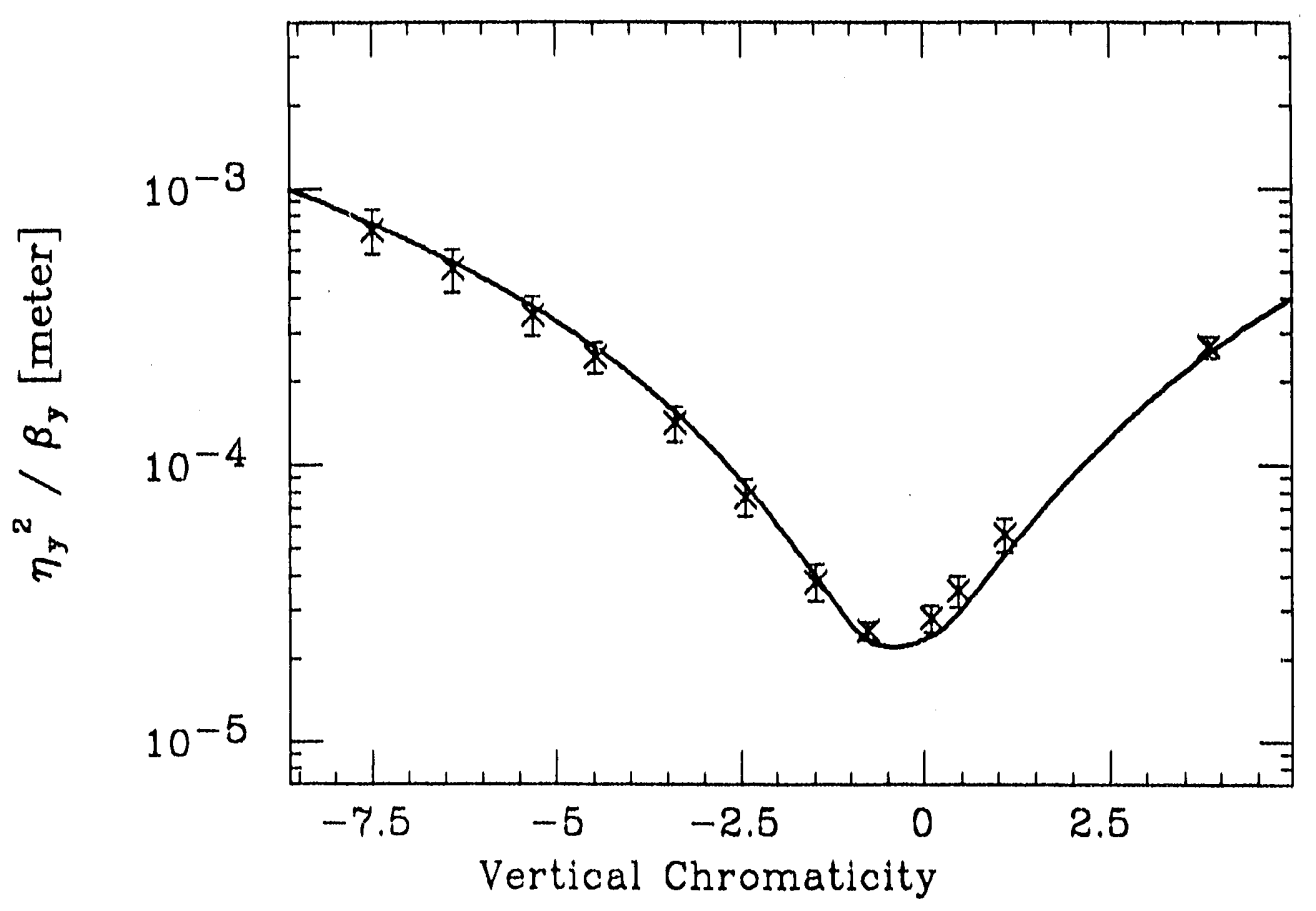

Fig. 2. Vertical dispersion versus $\xi_{y}$ for an uncorrected closed orbit.

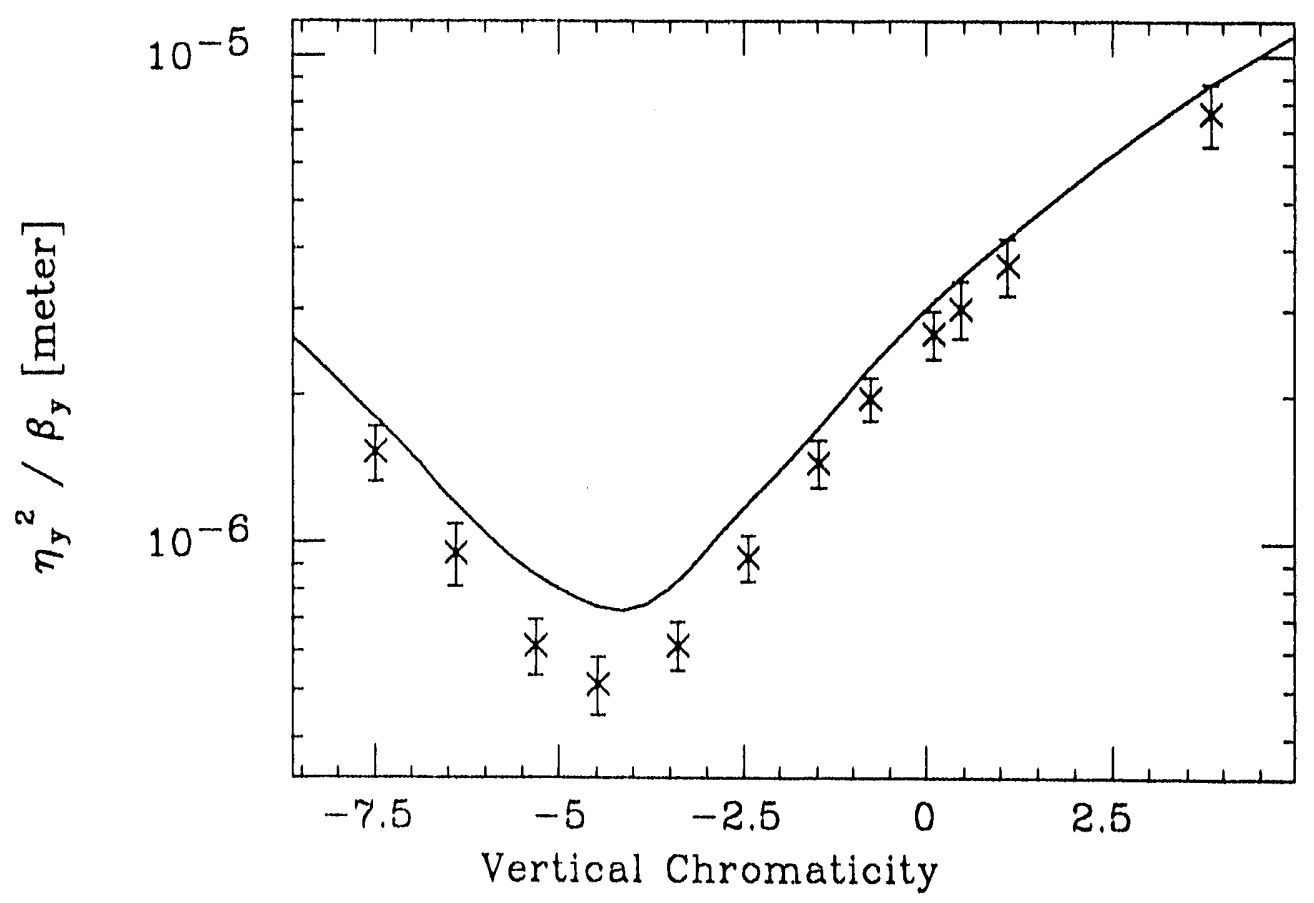

Fig. 3. Vertical dispersion versus $\xi_{y}$ for a closed orbit corrected with 20 correctors. 
are calculated using Eqs. (2.2.46), (2.2.49), and (2.2.53) and Eqs. (2.2.46), (2.2.50), and (2.2.55) for Figures 2 and 3, respectively.

As one can see, the character of the clispersion changes when the orbit is corrected. In the uncorrected case (Figure 2) the dispersion has a sharp minimum close to $\xi_{y}=0$, while in the corrected case (Figure 3 ), the minimum occurs near $\xi_{y}=-4.5$. We can understand this change in the following manner. The insertion regions in the NDR do not have local chromatic correction; they are compensated with sextupoles in the arcs. When the orbit is corrected, the minimum value of the dispersion occurs, not when the global chromaticity is corrected, but when the local values of $\xi$ and $\Delta$ are the smallest. This occurs when the sextupoles are used to locally compensate the arcs only, not both the insertion regions and the arcs.

Thus, when the chromaticity is zero, correcting the orbit will tend to increase the vertical dispersion relative to the closed orbit since $\xi_{\text {local }}$ and $\Delta_{\text {local }}$ grow. This is illustrated in Figure 4; here, the vertical enittance, scaled by the square of the closed orbit, has been plotted versus the number of correctors used to correct the orbit. Notice that, initially, the scaled rertical emittance increases rapidly as the orbit is corrected; it then pealis and slowly decreases. The initial increase occurs when, in Eq. (2.2.55), the insertion regions are separated from the arcs. Further correction then just subdivides the al'cs which has little effect. The line in Figure 4 is calculated using the estimates for a corrected orbit. Finally, again notice that Figure 4 is a plot of the vertical emittance normalized by the closed orbit; the actual vertical emittance tends to decrease as the orbit is corrected.

Before concluding this section, it is useful to contrast the behavior seen in Figure 4 with an example from the ALSS lattice. ${ }^{[129]}$ The ALS lattice differs from the NDR in that the ALS has twelve dispersion-free insertion regions rather than just two. Figure 5 is a plot of the scaled vertical cmittance due to quadrupole misalignments versus the number of correctors used to correct the orbit. Here, the scaled emittance contribution continues to increase rapidly' with the number of correctors. There are two reasons for this: first the fractional tune in the ALS is lower than in the NDR1, $\nu_{y}=8.18$ as compared to $\nu_{y}=3.275$, and thus the ALS is more sensitive to errors. Second, as mentioned, the ALS has twelve insertion regions and the chromatic correction is performed in the ares between these insertions. As the orbit is corrected, this non-local chromatic correction continues to be broken, causing the dispersion to increase relative to the closed orbit. 


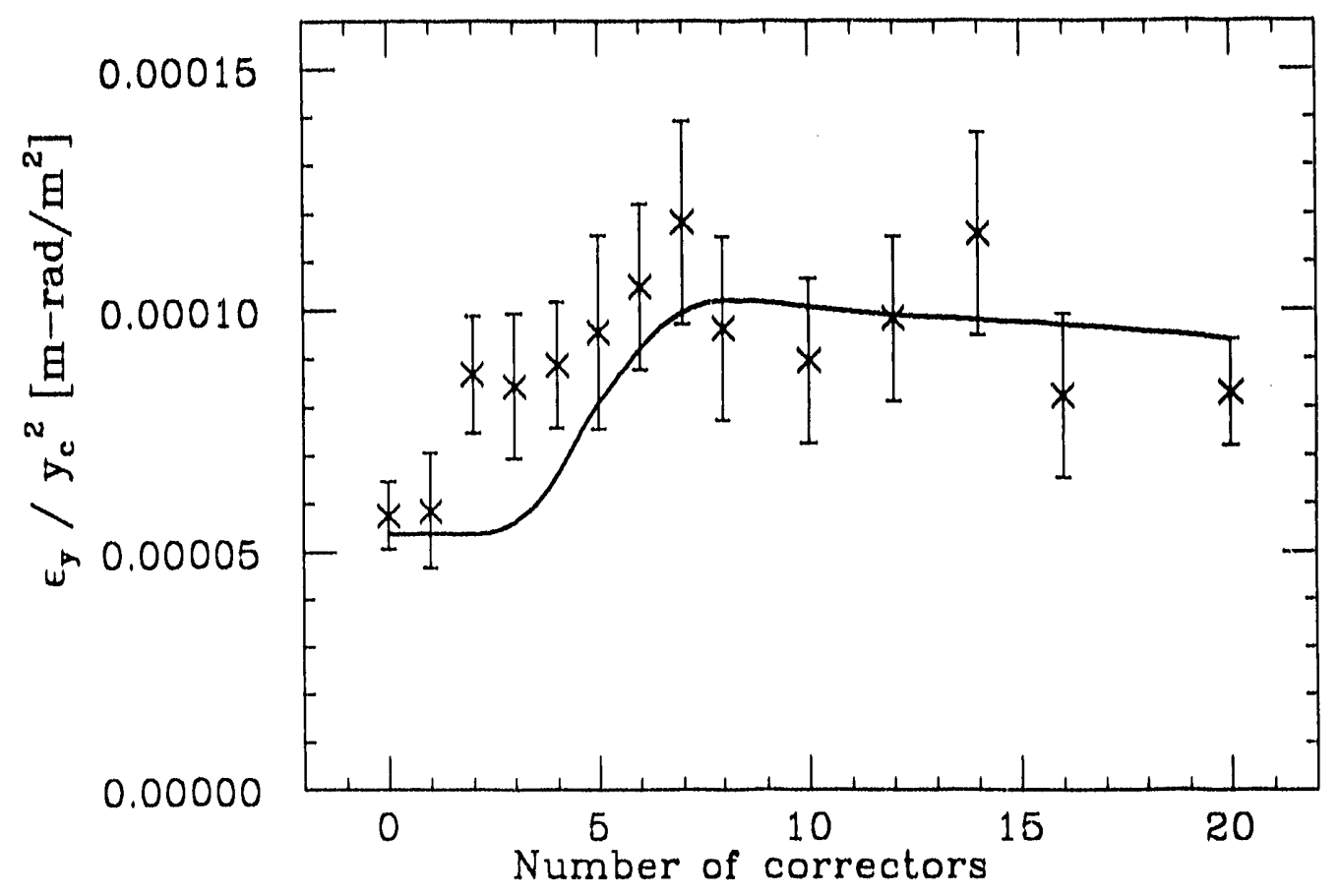

Fig. 4. $\epsilon_{y}$ due to vertical dispersion versus $N_{\text {corr }}$ in the NDR1 lattice with corrected chromaticity.

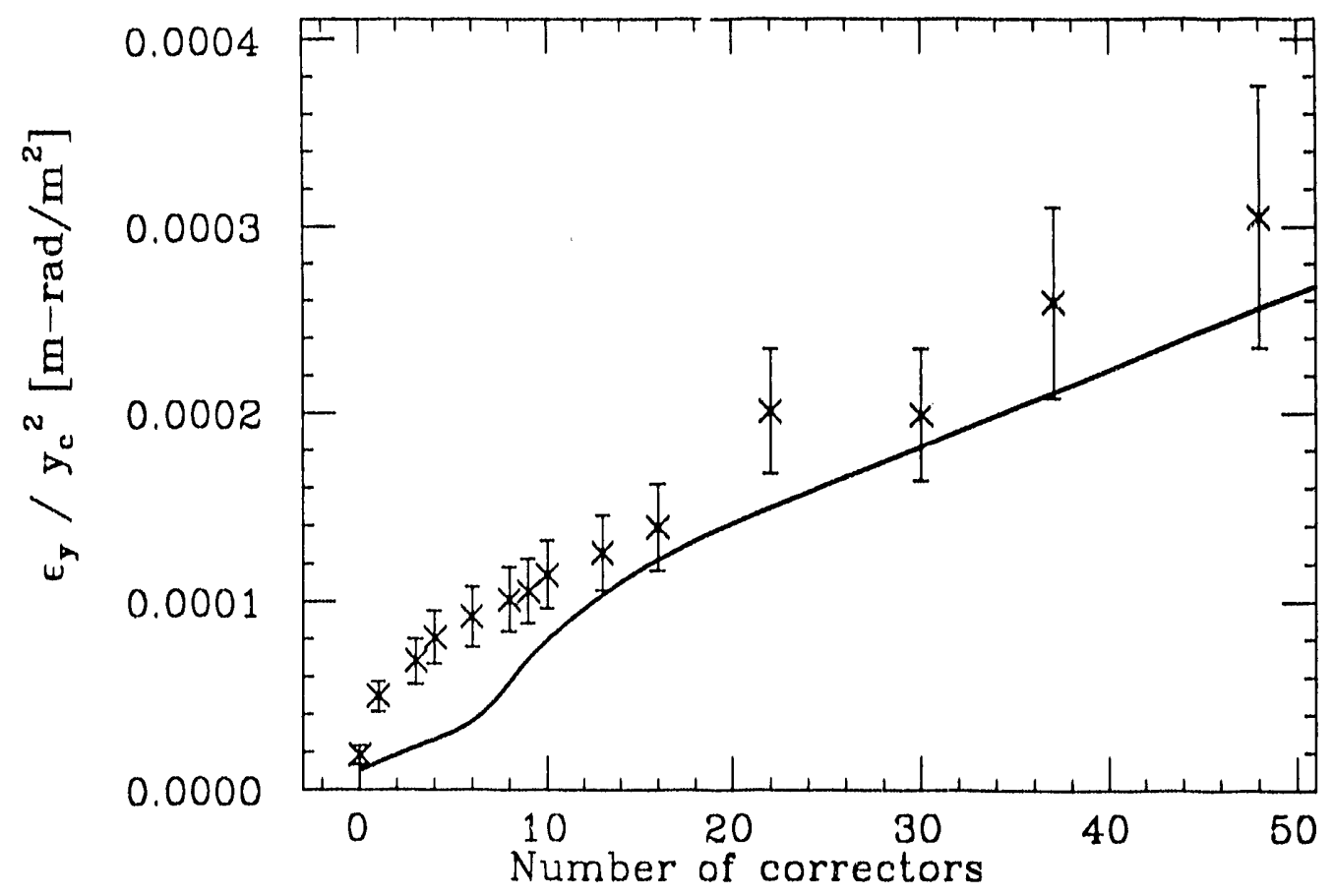

Fig. 5. $\epsilon_{y}$ due to vertical dispen'sion versus $N_{\text {corr }}$ in the ALS with corrected chromaticity. 


\subsubsection{Betatron Coupling}

In a conservative system, such as a proton storage ring, betatron coupling leads to "beats" where energy is transferred between the two transverse planes. An $e^{+} / e^{-}$ storage ring is not a conservative system; the synchrotron radiation provides both a source of noise and damping. Neglecting the vertical dispersion, only the horizontal plane is coupled to the noise soluce, while both planes are clamped. Thus, in an uncoupled ring, only the horizontal emittance is driven. Unfortunately, in the presence of coupling, the eigenvectors of the betatron motion rotate from the $x$ and $y$ axes so that both eigenmodes couple to the noise in the horizontal plane. Thus, in the case of weak betatron coupling, the vertical beam size is determined by both the projection of the "horizontal" emittance in the vertical plane and the contribution to the "vertical" emittance from the noise in the horizontal plane.

Much like the beam size due to the vertical dispersion, we can separate this increase into two contributions, one clue to a coupling which increases the projection of the six-dimensional emitiance into the vertical plane, and another clue to a fundamental increase in the verticil entillance. As before, the former effect will be referred to as the "local" contribution since it depends upon the local value of the coupling. In principal, this local coupling can be corrected at one location in the ring with four independent skew cluadrupoles; the four magnets can be used to uncouple the one-turn transport matrix at a specified location. Unfortunately, this does not remove the second effect, which arises from the "global" coupling; the coupling would need to be corrected at every bending magnet to fully remove this emittance contribution.

In this section, we will calculate the beam size increase due to both the local and the global coupling. Paralloling the cliscussion of the vertical dispersion, we will first calculate the effects of random errors and a non-zero closed orbit. Then, these analytic results will be compared with the results of simulations.

\subsubsection{Vertical Beam Size}

To calculate the rms equilibrium vertical beam size due to the linear betatron coupling, we will start from the equations of motion for a single particle, calculate the rms betatron motion, and finally, average over the ensemble of particles to find the rms beam size. Alternately, one could use the Fokker-Planck equation, but in 
many ways the more intuitive approach is appealing since it allows one to explicitly see the cause of the various contributions.

We will analyze the motion assuming that the coupling is weak and the vertical motion is much smaller than th horizontal. Thus, we can use the unperturbed horizontal motion to calculate the vertical. The equation for the vertical betatron motion was calculated, for the weali coupling limit, in Section 2.2.2:

$$
y_{13}^{\prime \prime}-K_{1} y_{\beta}=\left(K_{2} y_{c}-\tilde{K}_{1}\right) x_{\beta}
$$

where the effects of the syncluotron radiation have been neglected.

We want to calculate the change in $y_{\beta}$. Treating the magnets as delta-functions, the coupling adds a deflection $\Delta y^{\prime}=x_{\beta}\left(K_{2}^{\prime} y_{c}-\widetilde{K}_{1}^{\prime}\right) \Delta s$ to the vertical motion which is then exponentially damped by the radiation damping process. Thus, we can express the vertical motion as a sum over the deflections $\Delta y^{\prime}$

$$
y_{\beta}(s)=\int_{-\infty}^{s} d z \tilde{k}_{i}(z) x_{\beta}(z) e^{(z-s) a_{y} / c}\left[\sqrt{\beta_{y}(s) \beta_{y}(z)} \sin \left(\psi_{y}(s)-\psi_{y}(z)\right)\right] .
$$

Here, $\tilde{k}_{\mathrm{k}}$ is the coupling coufficint $\dot{h}_{(z)}(z)\left(\kappa_{2} y_{c}-\tilde{K}_{1}\right), \alpha_{y}$ is the vertical damping rate, and $c$ is the speed of light. In addition, the function enclosed in the brackets is the standard $R_{12}$ betatron matrix element which transforms a deflection $\Delta y^{\prime}$ at $z$ to a position $\Delta y$ at s.

At this point we need an expression for the $x$ betatron motion. The horizontal betatron motion is driven by ellergy fluctuations due to the synchrotron radiation; these are coupled to the horizontal plane through the dispersion. When a photon of energy $u$ is radiated, $x_{\beta}$ and $x_{\beta}^{\prime}$ clange by $\Delta x_{\beta}=\eta_{x} u / E_{0}$ and $\Delta x_{\beta}^{\prime}=\eta_{x}^{\prime} u / E_{0}$. For brevity, we will let $\eta_{x}^{\prime}$, and llus $\Delta_{a^{\prime}}{ }^{\prime}$, equal zero in the next two equations, but this assumption will be removed thereafler. In this case, the horizontal betatron motion is just a sum of displacements $\Delta$. is which are exponentially damped:

$$
x_{\beta}(s)=\sum_{\substack{i=-\infty \\\{\text { photans }\}}}^{s} \frac{u_{i}}{E_{0}} \eta_{x^{\prime}}\left(z_{i}\right) e^{\left(z_{1}-s\right) n_{x} / c}\left[\sqrt{\frac{\beta_{x}(s)}{\beta_{x}\left(z_{i}\right)}}\left(\cos \Delta \psi_{x}+\alpha_{x}\left(z_{i}\right) \sin \Delta \psi_{x}\right)\right] .
$$

Here, $u_{i}$ is a stochastic ranable equal to the energy of a photon radiated at $z_{i}$. In addition, $\Delta \psi_{x}=\psi_{x}(s)-\psi_{x}\left(z_{i}\right)$ and the function in brackets is the $R_{11}$ betatron 
matrix element which transforms a change in position $\Delta x$ at $z_{i}$ to a $\Delta x$ at $s$. Finally, we have assumed that the particle has been in the ring for infinite time and thus it has no memory of the initial amplitude and plase.

Now, we can use Eq. $(2.2 .61)$ to express $\mathrm{Eq} \cdot(2.2 .60)$ as a sum over photons $\xi_{i}$,

$$
y_{\beta}(s)=\sum_{\substack{i=-\infty \\ \text { photons }}}^{s} \frac{u_{i}}{E_{0}} \eta_{x}\left(z_{i}\right) \int_{z_{i}}^{s} d z e^{\left(z_{1}-z\right) a_{s} / c} R_{11 x}\left(z_{i}, z\right) e^{(z-s) \alpha_{y} / c} \hat{k}(z) R_{12 y}(z, s)
$$

The vertical beam size is now found by averaging the equilibrium value of $y_{\beta}^{2} / \beta_{y}$ over an ensemble of particles. The calculation simplifies since the radiation is a stochastic process, $\left\langle u_{i} u_{j}\right\rangle=\left\langle u_{i}^{2}\right\rangle \delta_{i}$. In addition, when performing the ensemble average, we can express the sum over photons as the integral of a rate of emission. Thus,

$$
\sum_{\substack{i=-\infty \\\{\text { photons }\}}}^{s}\left\langle u_{i}^{2}\right\rangle \Rightarrow \int_{-\infty}^{s} \frac{d z i}{c} \mathcal{N}\left\langle u^{2}\left(z_{i}\right)\right\rangle
$$

where this second moment of the photon distribution is given by Eq. (A.5.2). This yields a beam size of

$$
\begin{aligned}
& \frac{\sigma_{y}^{2}(s)}{\beta_{y}(s)}=\int_{-\infty}^{s} \frac{d z_{i}}{c} \mathcal{N} \frac{\left\langle u^{2}\left(z_{i}\right)\right\rangle}{E_{i}^{2}}\left[\int_{z_{1}}^{s} d l_{z} \dot{h}_{i}(z) e^{\left(z_{i}-z\right) a_{x} / c_{e} e^{(z-s) a_{y} / c} \sqrt{\beta_{x}(z) \beta_{y}(z)} \sin \Delta \psi_{y}}\right. \\
& \left.\times\left(\frac{\eta_{x}\left(z_{i}\right)}{\sqrt{\beta_{x}\left(z_{i}\right)}}\left(\cos \Delta \psi_{x}+\alpha_{x}\left(z_{i}\right) \sin \Delta \psi_{x}\right)+\eta_{x}^{\prime}\left(z_{i}\right) \sqrt{\beta_{x}\left(z_{i}\right)} \sin \Delta \psi_{x}\right)\right]^{2},
\end{aligned}
$$

where $\Delta \psi_{x}=\psi_{x}(z)-\psi_{x}\left(z_{i}\right)$ and $\Delta \psi_{y}^{\prime}=\psi_{y}(s)-\psi_{y}(z)$. Notice that we have included the contributions from both $\eta_{x}$ and $\eta_{x}^{\prime}$.

At this point, we assume that the tumes are far from the coupling resonances, $\nu_{x} \pm \nu_{y}=n$, and damping per turn is small compared to the tumes, $2 \pi\left(\nu_{x} \pm \nu_{y}\right) \gg$ $\alpha_{x} T_{0}, \alpha_{y} T_{0}$. After some algebra, that is explicitly displayed in Appendix C.2, we find contributions to the vertical beam size and enittance from both the sum and 
difference resonances of

$$
\begin{aligned}
& \frac{\sigma_{y}^{2}(s)_{\text {local }}}{\beta_{y}(s)}=\frac{\epsilon_{x}}{16}\left[\sum_{ \pm} \frac{\left|Q_{ \pm}(s)\right|^{2}}{\sin ^{2} \pi \Delta \nu_{ \pm}}-2 \operatorname{Re} \frac{Q_{+}(s) Q_{ \pm}^{\star}(s)}{\sin \pi \Delta \nu_{+} \sin \pi \Delta \nu_{-}}\right] \\
& \epsilon_{y}=\frac{C_{y} \gamma^{2}}{16 \mathcal{J}_{y} \oint G^{2} d s} \int_{0}^{C} d s \mathcal{H}_{x}\left|G^{\prime 3}\right|\left[\sum_{ \pm} \frac{\left|Q_{ \pm}(s)\right|^{2}}{\sin ^{2} \pi \Delta \nu_{ \pm}}+2 \operatorname{Re} \frac{Q_{+}(s) Q_{-}(s)}{\sin \pi \Delta \nu_{+} \sin \pi \Delta \nu_{-}}\right]
\end{aligned}
$$

where

$$
Q_{ \pm}(s)=\int_{s}^{s+C} d z \hat{k}(z) \sqrt{\beta_{x} \beta_{y}} e^{i}\left[\left(\psi^{\prime}(s) \pm \psi_{y}^{\prime}(s)\right)-\left(\psi_{x}(z) \pm \psi_{y}(z)\right)+\pi\left(\nu_{x} \pm \nu_{y}\right)\right] .
$$

Here, $\tilde{k}=\left(K_{2} y-\tilde{K}_{1}\right)$ and the sum over \pm denotes a sum over both the + term (sum resonance) and the - term (difference resonance) while $\Delta \nu_{+}=\nu_{x}+\nu_{y}$ and $\Delta \nu_{-}=\nu_{x}-\nu_{y}$. In addition, the ${ }^{*}$ is i sed to represent the complex conjugate and the operator "Re" yields the ral portion of the expression.

Equation (2.2.65) explicitly clisplays the physics described in the beginning of this section. The first expression represents the projection of the "horizontal" emittance into the vertical plane and the second expressions describes the contribution to the "vertical" emittance from the horizontal dispersion. This is analogous to the situation with the vertical clispersion where the projected vertical emittance is increased by the local value of the dispersion while the vertical emittance is increased by the average value of the dispersion.

This analogy can be talien further by noticing that the real part of $Q_{ \pm} / \sin \pi \Delta \nu_{ \pm}$ is analogous to the vertical clispersion with a phase advance of $\psi_{x} \pm \psi_{y}$ instead of $\psi_{y}$. In addition, the imaginary portion of $Q_{ \pm}(s) / \sin \pi \Delta \nu_{ \pm}$is analogous to $\eta_{y}^{*}(s)$, Eq. (2.2.41). Thus, $\left|Q_{ \pm}(s)\right|^{2} /$ sin" $^{2} \pi \Delta y_{ \pm}$is completely analogous to $\mathcal{H}_{x, y}(s)$. This analogy will be used in Section 2.2 .7 when we discuss correction of the coupling.

At this point, we should compare our result with the results of others. Equation (2.2.65) is similar to the result found in Ref. 26 where the expression was derived by solving the Fokker-Planck equation when close to the difference coupling resonance. The results differ in that. (1) the effect of the sum resonance and the cross terms between the sum and lifference resonances have been included, (2) the contribution to the vertical cmittance involves the average of the coupling coefficient 
around the ring while the contribution to the local beam size (projected emittance) just depends upon the local value of the coupling, and (3) the explicit form of the coupling coefficients differ slightly' In many references, including Refs. 26, 27, 46 , and 47 , the coupling coefficient is found by Fourier analyzing the coupling and choosing only the coefficient closest to the difference resonance. This is not valid in our case since we have assumed that the ring is far from both coupling resonances.

\subsubsection{RANDOM ERRORS}

Now we evaluate Eq. (2.2.65) for specific errors. The quadrupole rotational errors, sextupole misalignments, and the closed orbit are all independent. Thus, the square of the coupling function $k_{i}$ is

$$
\left\langle\tilde{k}(z) \tilde{k}_{(}\left(z^{\prime}\right)\right\rangle=4 K_{1}(z) K_{1}\left(z^{\prime}\right)\left\langle\Theta \Theta^{\prime}\right\rangle+\Lambda_{2}^{\prime}(z) \Lambda_{2}\left(z^{\prime}\right)\left(\left\langle y_{m} y_{m}^{\prime}\right\rangle+\left\langle y_{c} y_{c}^{\prime}\right\rangle\right)
$$

where primes have been used to indicate functions of $z^{\prime}$ rather than $z$. In the case of uncorrected coupling, we can quiclily craluate Eq. (2.2.65) to find the contribution from random quadrupole rotations and random sextupole misalignments. Specifically, we find

$$
\begin{aligned}
& \frac{\left\langle\sigma_{y}^{2}\right\rangle_{\text {local }}}{\beta_{y}}=\frac{\epsilon_{x}}{4} \frac{\left(1-\cos 2 \pi \nu_{x} \cos 2 \pi \nu_{y}\right)}{\left(\cos 2 \pi \nu_{x}-\cos 2 \pi \nu_{y}\right)^{2}} \sum_{q u u d}\left(K_{1} L\right)^{2} 4\left\langle\Theta^{2}\right\rangle \beta_{x} \beta_{y} \\
& \frac{\left\langle\sigma_{y}^{2}\right\rangle_{\text {local }}}{\beta_{y}}=\frac{\epsilon_{x}}{4} \frac{\left(1-\cos 2 \pi \nu_{x} \cos 2 \pi \nu_{y}\right)}{\left(\cos 2 \pi \nu_{x}-\cos 2 \pi \nu_{y}\right)^{2}} \sum_{y c x t}\left(\Lambda_{2} L\right)^{2}\left\langle y_{m}^{2}\right\rangle \beta_{x} \beta_{y}
\end{aligned}
$$

and

$$
\begin{aligned}
& \left\langle\epsilon_{y}\right\rangle=\frac{\epsilon_{x}}{4} \frac{\mathcal{J}_{x}}{\mathcal{J}_{y}} \frac{\left(1-\cos 2 \pi \nu_{x} \cos 2 \pi \nu_{y}\right)}{\left(\cos 2 \pi \nu_{x}-\cos 2 \pi \nu_{y}\right)^{2}} \sum_{y u a d}\left(\Lambda_{1} L\right)^{2} 4\left\langle\Theta^{2}\right\rangle \beta_{x} \beta_{y} \\
& \left\langle\epsilon_{y}\right\rangle=\frac{\epsilon_{x}}{4} \frac{\mathcal{J}_{x}}{\mathcal{J}_{y}} \frac{\left(1-\cos 2 \pi \nu_{x} \cos 2 \pi \nu_{y}\right)}{\left(\cos 2 \pi \nu_{x}-\cos 2 \pi \nu_{y}\right)^{2}} \sum_{s e x t}\left(\Lambda_{2} L\right)^{2}\left\langle y_{m}^{2}\right\rangle \beta_{x} \beta_{y}
\end{aligned}
$$

Here, the sum of $1 / \sin ^{2} \pi\left(\nu_{x} \pm \nu_{y y}\right)$ hats becu writhen in terms of $\cos 2 \pi \nu_{x}$ and $\cos 2 \pi \nu_{y}$ and we have simplified expression lic(. (2.2.(i9) with the cyulibrium horizontal emittence; these expressions are identical to those quoted in Rof. S2. 
difference resonances of

$$
\begin{aligned}
& \frac{\sigma_{y}^{2}(s)_{\text {local }}}{\beta_{y}(s)}=\frac{\epsilon_{x}}{16}\left[\sum_{ \pm} \frac{\left|Q_{ \pm}(s)\right|^{2}}{\sin ^{2} \pi \Delta \nu_{ \pm}}-2 \operatorname{Re} \frac{Q_{+}(s) Q_{-}^{\star}(s)}{\sin \pi \Delta \nu_{+} \sin \pi \Delta \nu_{-}}\right] \\
& \epsilon_{y}=\frac{C_{q} \gamma^{2}}{16 \mathcal{J}_{y} \oint G^{2} d s} \int_{0}^{C} d s \mathcal{H}_{x}\left|G^{\prime 3}\right|\left[\sum_{ \pm} \frac{\left|Q_{ \pm}(s)\right|^{2}}{\sin ^{2} \pi \Delta \nu_{+}}+2 \operatorname{Re} \frac{Q_{+}(s) Q_{-}(s)}{\sin \pi \Delta \nu_{+} \sin \pi \Delta \nu_{-}}\right]
\end{aligned}
$$

where

$$
Q_{ \pm}(s)=\int_{s}^{s+C} d z \tilde{k}(z) \sqrt{\beta_{x} \beta_{y}} e^{i\left[\left(\psi_{x}^{\prime}(s) \pm \psi_{y}(s)\right)-\left(\psi_{x}(z) \pm \psi_{y}(z)\right)+\pi\left(\nu_{x} \pm \nu_{y}\right)\right]} .
$$

Here, $\tilde{k}=\left(K_{2} y-\tilde{K}_{1}\right)$ and the sum over \pm denotes a sum over both the + term (sum resonance) and the - term (difference resonance) while $\Delta \nu_{+}=\nu_{x}+\nu_{y}$ and $\Delta \nu_{-}=\nu_{x}-\nu_{y}$. In addition, the ${ }^{\star}$ is used to represent the complex conjugate and the operator "Re" yields the real portion of the expression.

Equation (2.2.65) explicitly display's the physics described in the beginning of this section. The first expression represents the projection of the "horizontal" emittance into the vertical plane and the second expressions describes the contribution to the "vertical" emittance from the horizontal dispersion. This is analogous to the situation with the vertical dispersion where the projected vertical emittance is increased by the local value of the dispersion while the vertical emittance is increased by the average value of the dispersion.

This analogy can be talien further by noticing that the real part of $Q_{ \pm} / \sin \pi \Delta \nu_{ \pm}$ is analogous to the vertical clispersion with a phase advance of $\psi_{x} \pm \psi_{y}$ instead of $\psi_{y}$. In addition, the imaginary portion of $Q_{ \pm}(s) / \sin \pi \Delta \nu_{ \pm}$is analogous to $\eta_{y}^{*}(s)$, Eq. (2.2.41). Thus, $\left|Q_{ \pm}(s)\right|^{2} / \sin ^{2} \pi \Delta \nu_{ \pm}$is completely analogous to $\mathcal{H}_{x, y}(s)$. This analogy will be used in Scction 2.2.7 when we discuss correction of the coupling.

At this point, we should compare our result with the results of others. Equation (2.2.65) is similar to the result found in Ref. 26 where the expression was derived by solving the Folkicr-Planck equation when close to the difference coupling resonance. The results differ in that (1) the effect of the sum resonance and the cross terms between the sum and difference resonances have been included, (2) the contribution to the vertical mittance involves the average of the coupling coefficient 
around the ring while the contribution to the local beam size (projected emittance) just depends upon the local value of the coupling, and (3) the explicit form of the coupling coefficients differ slightly. In many references, including Refs. 26, 27, 46 , and 47 , the coupling coefficient is found by Fourier analyzing the coupling and choosing only the coefficient closest to the difference resonance. This is not valid in our case since we have assumed that the ring is far from both coupling resonances.

\subsubsection{RANDOM ERRORS}

Now we evaluate.Eq. (2.2.65) for specific errors. The quadrupole rotational errors, sextupole misalignments, and the closed orbit are all independent. Thus, the square of the coupling function $\hat{k}_{\mathrm{k}}$ is

$$
\left\langle\tilde{k}(z) \tilde{k}\left(z^{\prime}\right)\right\rangle=4 K_{1}^{\prime}(z) K_{1}^{\prime}\left(z^{\prime}\right)\left\langle\Theta \Theta^{\prime}\right\rangle+K_{2}^{\prime}(z) K_{2}\left(z^{\prime}\right)\left(\left\langle y_{m} y_{m}^{\prime}\right\rangle+\left\langle y_{c} y_{c}^{\prime}\right\rangle\right)
$$

where primes have been used to indicate functions of $z^{\prime}$ rather than $z$. In the case of uncorrected coupling, we can quickly evaluate Eq. (2.2.65) to find the contribution from random quadrupole rotations and random sextupole misalignments. Specifically, we find

$$
\begin{aligned}
& \frac{\left\langle\sigma_{y}^{2}\right\rangle_{\text {local }}}{\beta_{y}}=\frac{\epsilon_{x}}{4} \frac{\left(1-\cos 2 \pi \nu_{x} \cos 2 \pi \nu_{y}\right)}{\left(\cos 2 \pi \nu_{x}-\cos 2 \pi \nu_{y}\right)^{2}} \sum_{q u u d}\left(K_{1} L\right)^{2} 4\left\langle\Theta^{2}\right\rangle \beta_{x} \beta_{y} \\
& \frac{\left\langle\sigma_{y}^{2}\right\rangle_{\text {local }}}{\beta_{y}}=\frac{\epsilon_{x}}{4} \frac{\left(1-\cos 2 \pi \nu_{x} \cos 2 \pi \nu_{y}\right)}{\left(\cos 2 \pi \nu_{x}-\cos 2 \pi \nu_{y}\right)^{2}} \sum_{\text {sext }}\left(K_{2} L\right)^{2}\left\langle y_{m}^{2}\right\rangle \beta_{x} \beta_{y}
\end{aligned}
$$

and

$$
\begin{aligned}
& \left\langle\epsilon_{y}\right\rangle=\frac{\epsilon_{x}}{4} \frac{\mathcal{J}_{x}}{\mathcal{J}_{y}} \frac{\left(1-\cos 2 \pi \nu_{x} \cos 2 \pi \nu_{y}\right)}{\left(\cos 2 \pi \nu_{x}-\cos 2 \pi \nu_{y}\right)^{2}} \sum_{q u a d}\left(\Lambda_{1} L\right)^{2} 4\left\langle\Theta^{2}\right\rangle \beta_{x} \beta_{y} \\
& \left\langle\epsilon_{y}\right\rangle=\frac{\epsilon_{x}}{4} \frac{\mathcal{J}_{x}}{\mathcal{J}_{y}} \frac{\left(1-\cos 2 \pi \nu_{x} \cos 2 \pi \nu_{y}\right)}{\left(\cos 2 \pi \nu_{x}-\cos 2 \pi \nu_{y}\right)^{2}} \sum_{s e x t}\left(K_{2} L\right)^{2}\left\langle y_{m}^{2}\right\rangle \beta_{x} \beta_{y}
\end{aligned}
$$

Here, the sum of $1 / \sin ^{2} \pi\left(\nu_{x} \pm \nu_{y}\right)$ has been written in terms of $\cos 2 \pi \nu_{x}$ and $\cos 2 \pi \nu_{y}$ and we have simplified expression Eq. (2.2.(9) with the cquilibrium horizontal enittance; these expressions are identical to those quoted in Ref. 82. 
In addition, notice that the cross terms have been not included in Eq. (2.2.65). These terms add contributions of

$$
\begin{aligned}
& \frac{\left\langle\sigma_{y}^{2}\right\rangle_{\text {local }}}{\beta_{y}}=\frac{\epsilon_{x}}{16} \frac{\sin 2 \pi \nu_{y}}{2 \pi \nu_{y}} \frac{\sum_{\text {quadl }}\left(K_{1} L\right)^{2} 4 \Theta^{2} \beta_{x} \beta_{y}+\sum_{\text {sext }}\left(K_{2} L\right)^{2} y_{m}^{2} \beta_{x} \beta_{y}}{\sin \pi \Delta \nu_{+} \sin \pi \Delta \nu_{-}} \\
& \epsilon_{y}=\frac{\epsilon_{x}}{16} \frac{\mathcal{J}_{x}}{\mathcal{J}_{y}} \frac{\sin 2 \pi \nu_{x}}{2 \pi \nu_{x}} \frac{\sum_{q u a d}\left(K_{1} L\right)^{2} 4 \Theta^{2} \beta_{x} \beta_{y}+\sum_{\text {sext }}\left(K_{2} L\right)^{2} y_{m}^{2} \beta_{x} \beta_{y}}{\sin \pi \Delta \nu_{+} \sin \pi \Delta \nu_{-}} .
\end{aligned}
$$

Since these contributions are at least $1 / 2 \pi \nu_{x, y}$ smaller than the contributions from the individual resonances, they will be neglected in all future calculations.

\subsubsection{Orbit ERrors}

To calculate the effect of a closed orbit, we use Eq. (2.2.21) or Eqs. (2.2.18) and (2.2.20) for the correlation function of a corrected or uncorrected orbit. For an uncorrected orbit, we find

$$
\frac{\left\langle\sigma_{y}^{2}\right\rangle_{\text {local }}}{\beta_{y}} \approx \sum_{n, \Delta u_{ \pm}, \psi^{\prime}, n} \frac{\epsilon_{x}}{32 \sin ^{2} \pi \Delta \nu_{ \pm}} \frac{\left\langle y_{r}^{2}\right\rangle}{\beta_{y}} c_{n}\left|\int_{s}^{s+C} d z K_{2}^{\prime}(z) \beta_{y}(z) \sqrt{\beta_{x}(z)} e^{i \psi_{n}}\right|^{2}
$$

where the contribution to $\epsilon_{y}$ is similar, but it has as additional coefficient of $\mathcal{J}_{x} / \mathcal{J}_{y}$ and must be averaged around the ring. In addition, the sum over $\Delta \nu_{ \pm}$and $\psi_{n}$ is a sum over four terms: the tho values of $\Delta \nu_{ \pm}=\nu_{x} \pm \nu_{y}$ and the two values of $\psi_{n}$ associated with each value for $\Delta \nu_{ \pm}$. The values of $\psi_{n}$ are

$$
\psi_{n}=\psi_{x}+\left\{\begin{array}{rlrl}
\left(1+\frac{n}{\nu_{y}}\right) \psi^{\prime} y & \text { and }\left(1-\frac{n}{\nu_{y}}\right) \psi_{y}, & \Delta \nu_{ \pm}=\nu_{x}+\nu_{y} \\
-\left(1+\frac{n}{\nu_{y}}\right) \psi^{\prime} y & \text { and }-\left(1-\frac{n}{\nu_{y}}\right) \psi_{y}, & \Delta \nu_{ \pm}=\nu_{x}-\nu_{y}
\end{array} .\right.
$$

In the case of a corrected orbit, we find a form similar to Eq. (2.2.71), except that the integral is broken into segments by the correctors

$$
\frac{\left\langle\sigma_{y}^{2}\right\rangle_{\text {local }}}{\beta_{y}} \approx \sum_{\Delta \nu_{ \pm}, \psi^{\prime}} \frac{\epsilon_{x}}{32 \sin ^{2} \pi \Delta \nu_{ \pm}} \frac{\left\langle y_{c}^{2}\right\rangle}{\beta_{y}} \sum_{n_{c}}^{N_{\text {corr }}}\left|\int_{n_{c}}^{n_{c}+1} d_{z} K_{2}(z) \beta_{y}(z) \sqrt{\beta_{x}(z)} e^{i \psi}\right|^{2}
$$

where, again, the contribution to $c_{y}$ is similar. Here, the sum orer $\Delta \nu_{ \pm}$and $\psi$ is 
Table 5. $\epsilon_{y}$ from betatron coupling due to misalignments in the NDR1.

\begin{tabular}{|c|c|c|}
\hline Misalignment & Calc, $\epsilon_{y}[\mathrm{~m}$-rad $]$ & Simulated $\epsilon_{y}[\mathrm{~m}$-rad $]$ \\
\hline Random quad. $\Theta=0.5 \mathrm{mrad}$ & $6.00 \times 10^{-12}$ & $6.17 \pm 0.14 \times 10^{-12}$ \\
\hline Random sext. $y_{m}=150 \mu \mathrm{m}$ & $1.16 \times 10^{-11}$ & $1.11 \pm 0.02 \times 10^{-11}$ \\
\hline $\begin{array}{c}\text { Corrected closed orbit due to ranclom } \\
\text { quad. } y_{m}=150 \mu \mathrm{m} \text { and BPM } y_{m}=150 \mu \mathrm{m}\end{array}$ & $2.01 \times 10^{-12}$ & $2.6 \pm 0.3 \times 10^{-12}$ \\
\hline
\end{tabular}

the same as in the uncorrected case except $\psi$ is now

$$
\psi=\left\{\begin{array}{lll}
\psi_{x}+2 \psi_{y} & \text { and } \quad \psi_{x}, & \text { if } \Delta \nu_{ \pm}=\nu_{x}+\nu_{y} \\
\psi_{x}-2 \psi_{y} & \text { and } \quad \psi_{x}, & \text { if } \Delta \nu_{ \pm}=\nu_{x}-\nu_{y}
\end{array}\right. \text {. }
$$

The integrals in Eq. (2.2.73) are the same integrals one finds when using time dependant perturbation theory to calculate the effect of sextupoles on the betation motion. The similarity arises because. over a short segment, the closed orbit oscillates like a free betatron oscillation. It is important to emphasize that Eq. (2.2.73) describes an effect due to linear coupling - notice the resonant denominator in Eq. (2.2.73); it is not an effect of the third order resonances. Specifically, Eq. (2.2.73) is only valid when the closed orbit is broken into short segments (by correctors). Notice that if the orbit is broken at every' sextupole, Eq. (2.2.73) reduces to Eq. (2.2.69) which estimates the effect of random sextupole misalignments. Thus, for comparable orbits and misalignments $y_{c} \approx y_{m}$, the contribution to the beam size from the orbit will usually be less than the contribution from the misalignments since the orbit is typically correlated across many sextupoles.

Typically, when correcting the dynamic aperture, one adjusts the sextupole strength and placement so that the first order aberrations will cancel over the ring. For example, in the NDR, the cell phase advances are $\nu_{x \text { cell }} \approx 0.37$ and $\nu_{y \text { cell }} \approx 0.12$. This causes the first order geometric aberrations due to the sextupoles to cancel over an arc of roughly 8.5 cells. Unfortunately, when correcting the orbit, we break this cancellation scheme, and thus $\sigma_{y}^{2} / \beta_{y}$ normalized by the square of the closed orbit tends to grow.

\subsubsection{Simulations}

To verify the analytic results, the betatron coupling contributions to the vertical emittance were determined from simulations of landom alignment errors. Again, we 
used the program CEMIT, which calculates generalized synchrotron integrals, and, again, we used the NDR1 lattice; this is a lattice of the SLC NDR where the tunes have been changed to $\nu_{x}=8.375$ and $\nu_{y}=3.275$. Table 5 lists the results of simulating rotational misalignments in quadrupoles and vertical misalignments in sextupoles in the NDR1 lattice. As before, the misalignments are generated from a gaussian distribution with an $\mathrm{rms}$ of $0.5 \mathrm{mrad}$ and $150 \mu \mathrm{m}$ respectively. The calculated values are found using $\mathrm{E}_{(1} \cdot(2.2 .69)$. The simulated values are found by averaging the result of 1000 different random error distributions and the errors listed are the standard error of the average of the 1000 simulations; again, there is good agreement between the estimates and the simulation results.

In addition, Table 5 also lists results from simulating the effects of a corrected closed orbit where the results are found from twenty simulations. Here, the simulations included vertical quadrupole misalignments with an rms of $150 \mu \mathrm{m}$ and vertical BPM misalignments, also, with an rms of $150 \mu \mathrm{m}$. The resulting orbit was corrected using the twenty vertical dipole correctors in the NDR to minimize the rms of the simulated orbit. Before correction, the rms magnitude of the actual orbit was roughly $1.5 \mathrm{~mm}$; the cor'ection reduced this to $140 \mu \mathrm{m}$. In this case, the calculated result was found from Eq. (2.2.73). Again, the calculated estimate agrees well with the average of the simulations. Finally, notice that the contribution from the corrected orbit is less than the contribution due to similar misalignments in the sextupoles; as mentioned, this occurs because the orbit is still correlated over short segments.

Finally, in Figures 6 and 7 , the betatron coupling contribution to the vertical emittance, normalized by the square of the closed orbit, is plotted versus the number of orbit correctors used. The points plotted are generated by simulating random quadrupole and BPM misalignments in the NDR and the ALS as was done in Figures 4 and 5. The line is an approximation of Eq. (2.2.73) which we evaluated by assuming that correctors were evenly distributed in the ring. Notice that initially the normalized contribution incrases roughly linearly with the number of correctors. As mentioned, this occurs since lic cancellation is broken by the correctors. Of course, since the residual orbit is clecreased by the correction, the actual beam size contribution tends to decrease as the orbit is corrected. 


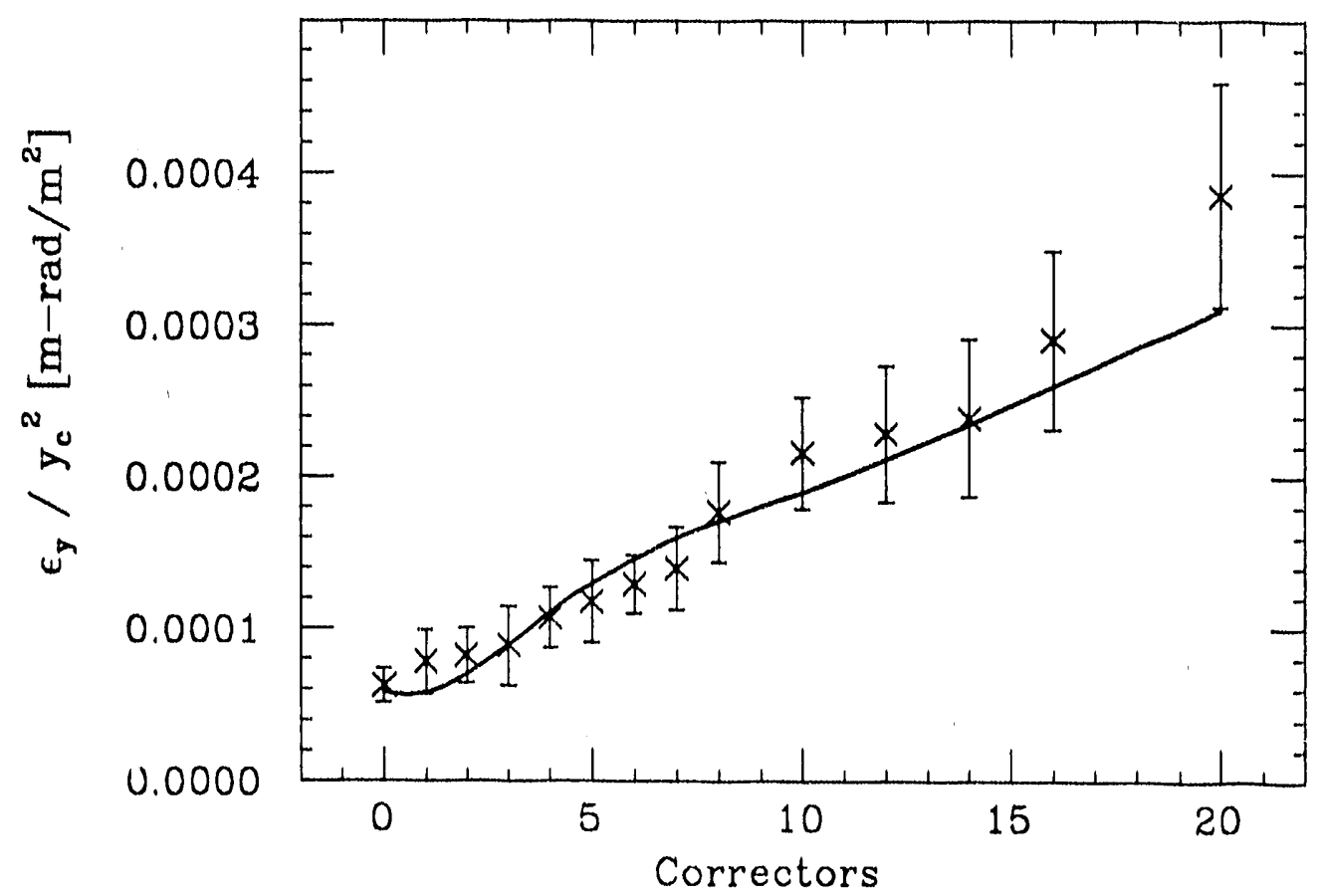

Fig. 6. $\epsilon_{y}$ clue to linear coupling versus $N_{\text {corr }}$ in the NDR1 lattice.

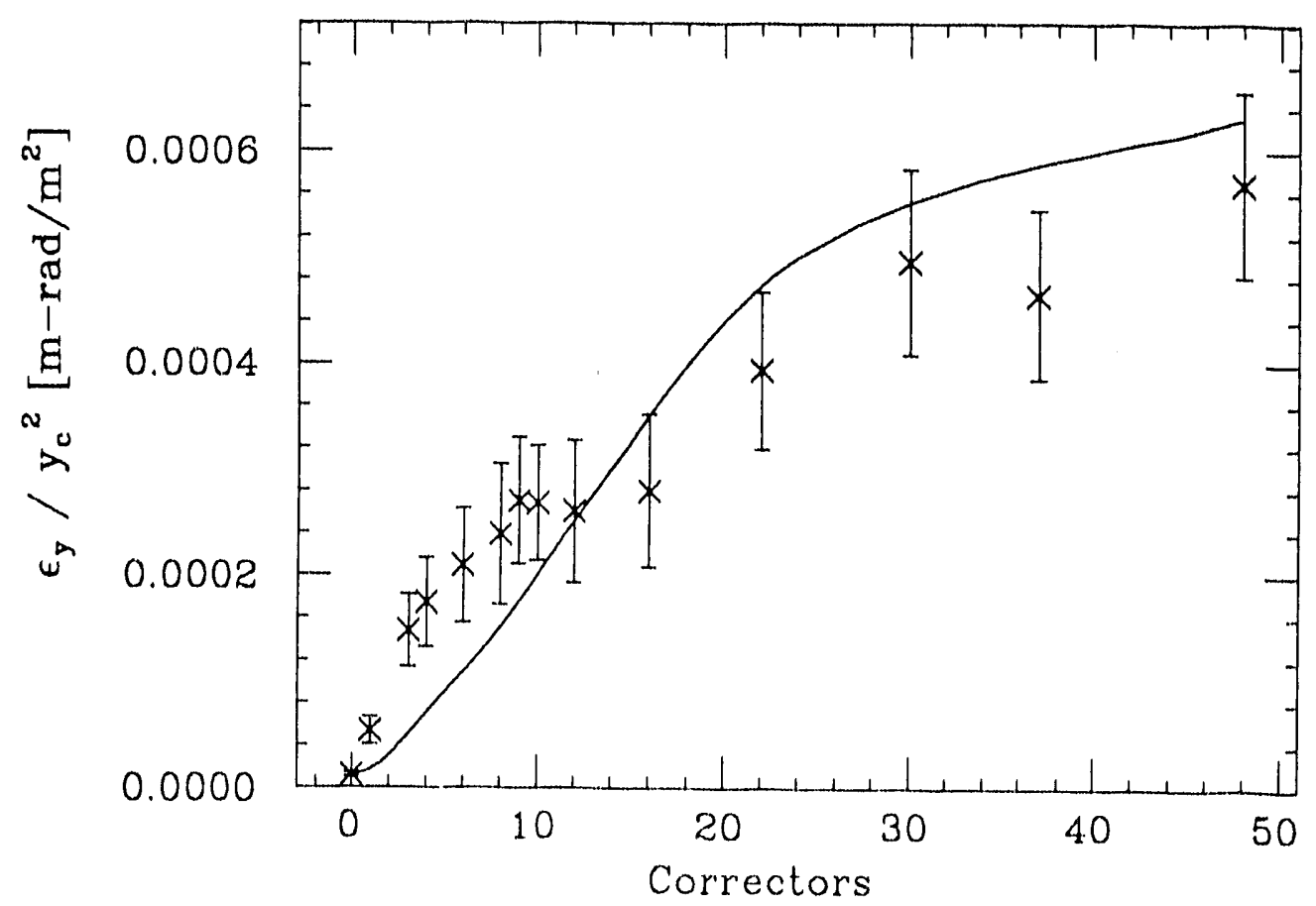

Fig. 7. $\epsilon_{y}$ due to lincar coupling versus $N_{\text {corr }}$ in the ALS. 


\subsubsection{Non-Linear COUPling EFfects}

We can also estimate the effects of the higher order coupling resonances. In this case, the equation for the restical betatron motion is

$$
y_{\beta}^{\prime \prime}+K_{1}^{\prime} y_{\beta}=K_{p}^{\prime} x_{\beta}^{m} y_{\beta}^{n}
$$

where $p=m+n$. Using perturbation theory, we would find a similar form for the increase in the vertical emittance except that the increase would depend upon higher powers of ${ }_{x}$ and the unperturbed vertical emittance. Because $\epsilon_{x}$ and $\epsilon_{y}$ are small, these effects will be negligible unless one is very close to the non-linear coupling resonances. A detailed analysis of these higher order coupling resonances can be found in Ref. 46.

Actually, there is one case where this higher order coupling could be significant. This occurs if the beam is very large when it is injected into the ring. Because of the large beam sizes, the wiclths of these higher order coupling resonances are larger. In simulations of a future damping ring lattice ${ }^{(00)}$ coupling has been observed after injecting the beam into the ring which was operating close to the sextupole difference resonance, $\nu_{x}-2 \nu_{y} \approx 0.03$. This is actually advantageous in this design since the vertical emittance damps faster when the beams are coupled; this occurs because $\mathcal{J}_{x}=1.6$ while $\mathcal{J}_{y}=1.0$ and thus there is more damping in the horizontal plane. Of course, one has to be sure that the beam becomes uncoupled before the horizontal emittance reaches its equilibrium value or the vertical emittance will never damp beyond this point.

\subsubsection{Synchrotron Motion}

So far, we have neglected the effects of the incoherent synchrotron motion; the basic synchrotron motion is discussed in Appendix A.3. In this section, we will discuss three effects that couple the transverse planes to the longitudinal. First, we will treat a direct synchro-betation coupling contribution that is completely analogous to the transverse betatron coupling discussed previously. Then, we will discuss the effect of the botation tune modulation due the synchrotron motion. Finally, we calculate the clange of the damping partitions during a synchrotron oscillation and the effect on the emittances and damping times. Although all these effects are manifestations of the synchrotron motion, they have different mechanisms, and thus we treat them separately. 


\subsubsection{Synchro-Betatron Coupling}

The direct synchro-betatron coupling is a coupling of the transverse and the longitudinal phase spaces through the longitudinal position $z$ in the bunch. The theory of the coupling was first developed in Ref. 80 . Since then there have been numerous refinements and generalizations; a good review can be found in Ref. 114. Unfortunately, most of these theories use a symplectic approach that is suitable for strong coupling but is unnecessarily complex for weak coupling and flat beams.

The synchro-betatron coupling is completely analogous to the transverse betatron coupling described in Section 2.2.5. In a proton ring, the coupling will leads to "beats" where the transverse planes and the longitudinal plane exchange energy. Again, the situation is slightly different in an electron ring because of the synchrotron radiation. Thus, there are two contributions: one due to the projection of the longitudinal emittance in the vertical plane, and another due to a fundamental increase in the vertical emittance. In theor'y, the former can be corrected, even after the beam has been extracted from the storage ring. In contrast, the emittance contribution must be corrected by correcting the coupling sources in the ring.

At low currents, the synchro-betatron coupling is induced in the RF cavities. The primary causes are either clispersion in the cavities, an angular misalignment between the RF accelerating ficld and the closed orbit, or RF deflecting fields. In addition, the coupling can be caused by transver'se waliefields due to a non-zero closed orbit or longitudinal wakefields in regions of dispersion. Here, we will only treat the effects of dispersion in the RF cavities and angular misalignments of the cavities although the expressions are trivial to generalize; the wakefield effects are calculated in Section 2.3.4.

To estimate these synchro-betatron contributions, we will derive an expression for the coupling due to a sinusoidal $R F$ voltage when off resonance. The derivation will parallel that of the transverse betatron coupling, allowing us to use results directly from Section 2.2.5.1. As before, we will analyze the motion assuming that the coupling is weak and the vertical motion is much smaller than the longitudinal; this is equivalent to the condition $c_{y} \ll \sigma_{\mathrm{e}} \sigma_{z}{ }^{[13]}$ and is satisfied in most storage rings.

Paralleling Section 2.2.5.1, we start by calculating the change in $y_{\beta}$ on passing through a cavity, where $y_{\beta}$ is the betatron component of the vertical motion. The energy gain in a cavity is a function of $z$, the longitudinal position in the bunch. 
Thus, if there is vertical dispersion or the accelerating field is not aligned to the trajectory, there will be a change in $y_{\beta}$ that depends upon $z$. Treating the cavities as delta-functions, we find

$$
\Delta y_{\beta}=\eta_{y} \frac{\Delta E_{\mathrm{RF}}(z)}{E_{0}} \quad \text { and } \quad \Delta y_{\beta}^{\prime}=\left(\eta_{y}^{\prime}+\Theta\right) \frac{\Delta E_{\mathrm{RF}}(z)}{E_{0}}
$$

where $\Theta$ is the angular misalignment of the cavity fields with respect to the closed orbit and $\Delta E_{\mathrm{RF}}$ is the diffcrcnce between the energy gain of a particle at longitudinal position $z$ and the energy gain of the synchronous particle. Note that the synchronous energy gain does not directly affect the beam size or the emittance; it simply replaces the average energy lost to radiation.

If we assume a $R F$ sinusoiclal voltage, $\Delta E_{\mathrm{RF}}$ is

$$
\frac{\Delta E_{\mathrm{RF}}(z)}{E_{0}}=\frac{e V_{\mathrm{RF}}}{E_{0}}\left(\sin \left(z k_{\mathrm{RF}}+\phi_{s}\right)-\sin \phi_{s}\right)
$$

where, $k_{\mathrm{RF}}$ is the wavenumber for the accelerating field $k_{\mathrm{RF}}=2 \pi / \lambda_{\mathrm{RF}}, \phi_{s}$ is the synchronous phase, and $V_{R F}$ is the accelerating voltage. This expression can be expanded as a power series in z, yielding

$$
\Delta y_{\beta}=\sum_{n=1}^{\infty} f_{n} z^{n} L_{\mathrm{cav}} \quad \text { and } \quad \Delta y_{\beta}^{\prime}=\sum_{n=1}^{\infty} g_{n} z^{n} L_{\mathrm{cav}}
$$

where $L_{\text {cav }}$ is the length of the RF cavities and

$$
f_{n}=\eta_{y} \frac{e V_{\mathrm{RF}}}{L_{\mathrm{cav}} E_{0}} \frac{k_{\mathrm{RF}}^{n}}{n !} \begin{cases}(-1)^{(n-1) / 2} \cos \phi_{s}, & \text { if } n \text { is odd } \\ (-1)^{n / 2-1} \sin \phi_{s}, & \text { if } n \text { is even }\end{cases}
$$

and $g_{n}$ is similar except it has a coefficient of $\eta_{y}^{\prime}+\Theta$ instead of $\eta_{y}$.

Now, we can express the vertical motion as a sum over the changes $\Delta y_{\beta}$ and $\Delta y_{\beta}^{\prime}$ in an equation analogous to $\mathrm{E}_{(} \cdot(2.2 .60)$ :

$$
y_{\beta}(s)=\sum_{n=1}^{\infty} \int_{-\infty}^{s} d s^{\prime} e^{\left(s^{\prime}-s\right) n_{y} / c} z^{n}\left(s^{\prime}\right)\left[f_{n} R_{y 11}\left(s^{\prime}, s\right)+g_{n} R_{y 12}\left(s^{\prime}, s\right)\right]
$$

where $R_{y 11}$ and $R_{y 12}$ are the standard betatron transport matrix elements that map a position offset or a deflection at. location $s^{\prime}$ to a position offset $\Delta y$ at location $s$. 
Next, we need an expression, antlogous to Eq. $(2,2.61)$, for the longitudinal position $z$, which is driven by the energy fluctuations due to the synchrotron radiation. In the smooth approximation of $\mathrm{Ec}$. (A.3.9), this can be written

$$
z(s)=\sum_{\substack{i=-\infty \\\{\text { photons }\}}}^{s} \frac{u_{i}}{E_{0}} \frac{\alpha_{m} C}{2 \pi \nu_{s}} \epsilon^{\left(s_{1}-s\right) \alpha_{c} / c} \sin \left(2 \pi \nu_{s} \frac{s-s_{i}}{C}\right)
$$

where, $u_{i}$ is a stochastic variable equal to the energy of a photon radiated at $s_{i}, \alpha_{\epsilon}$ is the longitudinal damping rate, $\alpha_{m}$ and $\nu_{s}$ are the momentum compaction factor and the synchrotron tune, and $C$ is the ring circumference. Note that we have neglected the nonlinearity of the synchrotron motion. Strictly, this should be included in Eq. (2.2.81) but we will neglect it. In general, the nonlinearity of the synchrotron motion will reduce the importance of the nonlinear resonances ( $\nu_{s}$ is smaller).

At this point, we can calculate the vertical beam size and the emittance dilution by following the procedure described in Siction 2,2.5.1. The only difference is that now we need to include the nonlinearity in $z$. This is easily handled with the identity

$$
\int_{-\infty}^{s} d s^{\prime} f\left(s^{\prime}\right)\left(\int_{-\infty}^{s^{\prime}} d s_{i} u\left(s_{i}\right)\right)^{n}=n \int_{-\infty}^{s} d s_{i} u\left(s_{i}\right)\left(\int_{-\infty}^{s_{i}} d s_{i}^{\prime} u\left(s_{i}^{\prime}\right)\right)^{n-1} \int_{s_{i}}^{s} d s^{\prime} f\left(s^{\prime}\right)
$$

which can be verified by integrating lyy parts. In addition, when calculating the ensemble average of $y_{\beta}^{2} / \beta_{y}$, one finds higher moments of the photon distribution. Since many photons are emitted, we can use the Central Limit Theorem to express

$$
\left\langle\left(\int_{-\infty}^{s_{i}} d s_{i}^{\prime} u\left(s_{i}^{\prime}\right) f\left(s_{i}^{\prime}\right)\right)^{2 n-2}\right\rangle=\frac{(2 n-2) !}{2^{n-1}(n-1) !}\left(\int_{-\infty}^{s_{1}} d s_{i}^{\prime}\left\langle u^{2}\left(s_{i}^{\prime}\right)\right\rangle f^{2}\left(s_{i}^{\prime}\right)\right)^{n-1},
$$

where $f$ is the integrand and we have assumed a gaussian distribution suitable for low beam intensity.

Thus, assuming that one is far from resonance, we find the results

$$
\begin{aligned}
\frac{\sigma_{y}^{2}(s)_{\text {local }}}{\beta_{y}(s)}=\frac{n \sigma_{z}^{2 n}(2 n-2) !}{4^{n+1}(n-1) !}[ & \frac{\left|S_{ \pm}^{(n)}(s)\right|^{2}}{\sin ^{2} \pi\left(n \nu_{s} \pm \nu_{y}\right)} \\
& \left.-2 \operatorname{Re} \frac{S_{+}^{(n)}(s) S_{-}^{(n) \star}(s)}{\sin \pi\left(n \nu_{s}+\nu_{y}\right) \sin \pi\left(n \nu_{s}-\nu_{y}\right)}\right]
\end{aligned}
$$


and

$$
\begin{aligned}
\epsilon_{y}=\frac{n \sigma_{z}^{2 n}(2 n-2) !}{4^{n+1}(n-1) !} \frac{n \mathcal{J}_{c}}{\mathcal{J}_{y}} \int_{0}^{C} \frac{d s}{C}\left[\sum_{ \pm} \frac{\left|S_{ \pm}^{(n)}(s)\right|^{2}}{\sin ^{2} \pi\left(n \nu_{s} \pm \nu_{y}\right)}\right. \\
\left.+2 \operatorname{Re} \frac{S_{+}^{(n)}(s) S_{-}^{(n)}(s)}{\sin \pi\left(n \nu_{s}+\nu_{y}\right) \sin \pi\left(n \nu_{s}-\nu_{y}\right)}\right]
\end{aligned}
$$

where

$$
\begin{aligned}
S_{ \pm}^{(n)}(s)=\int_{s}^{s+C} d s^{\prime} & \left(\frac{\left(\alpha_{y}+i\right)}{\sqrt{\beta_{y}}} f_{n}+\sqrt{\beta_{y}} g_{n}\right) \\
& \times e^{i\left[2 \pi \mu \nu_{s}\left(s-s^{\prime}\right) / C \pm\left(\psi_{y}(s)-\psi_{y}\left(s^{\prime}\right)\right)+\pi\left(n \nu_{s} \pm \nu_{y}\right)\right]},
\end{aligned}
$$

and the coefficients $f_{n}$ and $g_{n}$ are defined in Eq. (2.2.78). Note that we have neglected the cross terms between resonance orders. Although this is not valid when there are many contributions of ecual magnitude, usually the nonlinear contributions are only significant. when very close to the nonlinear resonances; we will discuss this shortly. Finally, nolice that in Eq. (2.2.85) we have assumed that the photons are radiated uniformly around the ring. This is acceptable because the emittance contribution, $\mathrm{Eq} \cdot(2.2 .85)$, is small.

Unlike the situation for the transverse betatron coupling, the cross term in Eq. (2.2.85) is important; it will tend to cancel the two main terms, reducing the emittance contribution. This occurs because the synchrotron tune is small. The cross term in Eq. (2.2.85) depends upon the average of an oscillating term $e^{i 4 \pi \nu_{o} s / C}$. Since $\nu_{s}$ is small, the oscillating term is nearly constant and the cross term is large. In fact, in an isochronous jing, where the synchrotron tune equals zero and the longitudinal distribution in $z$ is frozen, Eq. (2.2.85) predicts zero emittance dilution; the cross term exactly cancels the nain terms. This differs from the transverse case where the cross term is small since it depends on the average of $e^{i 4 \pi \nu_{x} s / C}$, which oscillates rapidly.

At this point, we need to discuss the effect of the nonlinearities. First. the nonlinearitises are (usually) wealice than the linear contribution and thus arc mil! important when on resonance. To analyze these effects correctly, we should fulluw the procedure of Refs. 80 and 113, where the coupling is analyzed on resonance. 
Table 6. Longitudinal parameter's for the SLC NDR and the ALS.

\begin{tabular}{|c|c|c|}
\hline & SLC NDR & ALS \\
\hline$f_{\mathrm{RF}}$ & $714 \mathrm{MHz}$ & $500 \mathrm{MHz}$ \\
\hline$V_{\mathrm{RF}}, E_{0}^{\prime}$ & $1 \mathrm{MV}, 1.2 \mathrm{GeV}$ & $1.5 \mathrm{MV}, 1.5 \mathrm{GeV}$ \\
\hline$\nu_{y}, \nu_{s}$ & $3.17,0.01$ & $8.18,0.008$ \\
\hline$\sigma_{z}, \beta_{y}$ & $5 \mathrm{~mm}, 2 \mathrm{~m}$ & $4 \mathrm{~mm}, 5 \mathrm{~m}$ \\
\hline
\end{tabular}

Instead, we will simply note that these nonlinear resonances are usually very narrow and, provided that the resonance is high order, we can neglect them. This occurs because damping rings and synchrotron light sources tend to have bunches short compared to the RF wavelength, and thus the nonlinear dependence of the forces on $z$ is small.

Finally, we will illustrate the importance of these effects by estimating their magnitude in the SLC NDR and ALS storage rings. Table 6 lists the relevant equilibrium longitudinal parameter's for both rings. Using these values and Eq. (2.2.84), we find a contribution from the linear coupling due to $\eta_{y}^{\prime}$ or an angular misalignment of:

$$
\frac{\sigma_{y}^{2}}{\beta_{y}} \lesssim 5 \times 10^{-4}\left(\eta_{y}^{\prime}+\Theta\right)^{2},
$$

for both the SLC NDR and the ALS. 'This is a very loose tolerance, much looser than the effect of random dispersion in the bending magnets. Furthermore, because the synchrotron tunes are so sma!l, the nonlinear resonances can be safely ignored; the resonances would be extremely high order ( 220 th order).

\subsubsection{Tune Modulation}

The synchrotron motion also modulates the betatron tunes. This is due to both the chromatic dependance of the betatron tunes and the effect of the space charge tune shift; the space charge tune shift depends upon the local bunch density which is a function of the longitudinal position $z$, see Section 2.3.3. The chromaticity modulates the betatron tunes at the sy'uchrotron frequency while the space charge modulates the tunes at twice this rate.

This slow modulation will generate sidebands separated by $\nu_{s}$ or $2 \nu_{s}$ around the betatron tunes. These sidcbands can thon overlap nearby resonances, increasing 
the beam size and emittance. We can estimate the importance of this nonlinear coupling from the magnitude of the sidebands. Initially, we will only consider the effect of the chromaticity. In the smooth approximation, the equation for a betatron oscillation can be written

$$
\frac{d^{2} y}{d \theta^{2}}+\left(\nu_{y}+\xi_{y} \delta_{0} \cos \left(\nu_{s} \theta+\theta_{0}\right)\right)^{2} y=0
$$

where $\delta_{0}$ is the amplitude of the energy synchrotron oscillation and $\theta_{0}$ is the initial phase.

Since $\nu_{s}$ is much less than $\nu_{y}$, the solution for the vertical motion can be found using the WKB approximation. This can be expressed in terms of the frequency components

$$
y(\theta)=\operatorname{Re}\left[y_{0} e^{i \nu_{y} \theta} \sum_{k=-\infty}^{\infty} i^{k} e^{i k \nu_{\Delta} \theta} J_{k}\left(\frac{\xi_{y} \delta_{0}}{\nu_{s}}\right)\right]
$$

where $J_{k}$ are Bessel functions of integral order and $y_{0}$ is the initial amplitude. One can estimate the magnitude of these sidebands using an asymptotic expansion of the Bessel functions for large orclers:

$$
J_{k}\left(i l^{\prime}\right) \sim \frac{1}{\sqrt{2 \pi k}}\left(1.36 \frac{x}{k}\right)^{k}
$$

This shows that the Bessel functions decrease rapidly for orders $k \gtrsim x$ and thus it confirms the intuitively obvious fact that sidebands, separated from the betatron tune by more than the tune shift, are small.

Now, we can calculate the coupling due to the sidebands by replacing $\nu_{y}$ in Eqs. (2.2.65), (2.2.84), and (2.2.85) with $\nu_{y} \pm k \nu_{s}$ and multiplying the result by the square of the Bessel function coefficient in Eq. (2.2.89). Since the chromaticity is usually corrected to the order of mity while the energy spread is the order of $10^{-3}$, this effect tends to be negligible.

At this point, we can follow the same procedure to calculate the effect of the space charge tune shift. In contrast to the effect of the chromaticity, this effect may become significant in future rings since the tune modulation increases as the vertical beam size decreases. Assuming a gaussian longitudinal distribution and linear synchrotron oscillations, the exalion for a betatron oscillation, in the smooth 
approximation, is:

$$
\frac{d^{2} y}{d \theta^{2}}+\left(\nu_{y}+\Delta \nu_{\mathrm{gc}} \epsilon^{-z_{0}^{2} \cos ^{2}\left(\nu_{0} \theta+\phi_{0}\right) / 2 \sigma_{2}^{2}}\right)^{2} y=0
$$

where $\Delta \nu_{\mathrm{sc}}$ is the space charge tune shift at the center of the bunch, $z_{0}$ is the amplitude of the synchrotron oscillation, and $\phi_{0}$ is the initial phase.

Again, using the WKB approxination, the solution for the vertical motion can be written

$$
y(\theta)=\operatorname{Re}\left[y_{0} e^{i \bar{\nu}_{\nu} \theta} \prod_{l=1}^{\infty} \sum_{k=-\infty}^{\infty} i^{k} e^{i k l 2 \nu_{s} H} J_{k}\left(\frac{i^{l} \Delta \nu_{\mathrm{sc}} e^{-z_{0}^{2} / 4 \sigma_{z}^{2}}}{\nu_{s} l} J_{l}\left(\frac{i z_{0}^{2}}{4 \sigma_{z}^{2}}\right)\right)\right]
$$

where $\overline{\nu_{y}}$ is the average tune which now depends upon the synchrotron amplitude:

$$
\overline{\nu_{y}}=\nu_{y}+\Delta \nu_{\mathrm{sc}} \epsilon^{-z_{0}^{2} / 4 \sigma_{2}^{2}} J_{0}\left(\frac{i z_{0}^{2}}{4 \sigma_{z}^{2}}\right)
$$

Obviously, this is much more complicated than the tune modulation due to the chromaticity, although in general, the same conclusion applies: the sidebands are small when greater than the amplitude of modulation. However, now there is one important difference: the space charge modulation causes a tune spread, as specified by Eq. (2.2.93), in addition to the sidebands. This will further reduce the available non-resonant tune space.

\subsubsection{Modulation of the Damping Partitions}

During the synchrotron oscillation, the particle energy changes, changing the damping times and the quantum excitation; ${ }^{[30,121]}$ this then causes the expected sirigle particle amplitude to oscillate. Uswally, the strongest energy dependence is due to the energy dependence of the damping partitions. For example, at injection in the SLC NDR, a particle with an encrgy $E=E_{0}+\sigma_{r}$, where $\sigma_{f}$ is the rms energy spread of $1 \%$, will have a horizontal damping rate that is $16 \%$ slower than a particle with the design energy"; $d \mathcal{J}_{x} / d E$ is negative. Of course, since the synchrotron oscillation frequency is much larger than the damping rates, the average damping rate is unchanged, but the fluctuating single particle amplitude could change the equilibrium beam emittance. 
In Ref. 121, an expression is clerived for the equilibrium single particle invariant as a function of the synchrotron phase and amplitude:

$$
J=J_{0} \exp \left(-2 \Delta E \frac{d \mathcal{J}}{d E} \frac{\cos \Omega t+\phi_{0}}{\tau \Omega}\right)
$$

where $J_{0}$ is unperturbed equilibrium invariant, $\mathcal{J}$ is the damping partition, $\Omega$ is the synchrotron frequency, and $\tau$ is the synchrotron radiation damping time.

Now, to determine the rms emittance increase in the beam, we need to integrate this expression over the beam distribution of synchrotron phase and amplitude. The expression within the brackets is usually small and thus we can expand the exponential. After integrating over the distribution, we find

$$
\epsilon=\epsilon_{0}\left[1+\left(\frac{\sigma_{\epsilon}}{\Omega \tau} \frac{d \mathcal{J}}{d E}\right)^{2}+\cdots\right] .
$$

There is no linear contribution to the beam emittance and the second order contribution tends to be small. In both the SLC NDR and the ALS, the relative emittance increase due to this effect is the order of $10^{-8}$.

\subsubsection{Correction}

In this section, we will discuss reduction and correction of the vertical dispersion and the betatron coupling. The simplest way to reduce these effects is to decrease the sensitivity of the ring to the errors. The most obvious method of doing this is to reduce the resonant denominators $1 / \sin ^{2} \pi \nu_{y}$ or $1 / \sin ^{2} \pi \Delta \nu_{ \pm}$which appear in all the dispersion and coupling formulas

In addition to decreasing the sensitivity to the errors, one can correct these effects directly. Specifically, we will first calculate the amount one can correct the vertical dispersion with a pair of correctors, typically skew quadrupoles, separated by ninety degrees in phase. Then, using the analogy between the vertical dispersion and the coupling functions that was noted in Section 2.2.5.1, we will apply our results to the correction of the betatron coupling. Finally, we will compare these analytic estimates with the results of simulations.

Notice that we have not mentioned correcting the synchro-betatron coupling. This is corrected by correcting line orbit or the dispersion at the cavities and thus falls under the vertical dispersion discussion, but, since the synchro-betatron coupling is small, as calculated in Eq. (2.2.87), we will not discuss it directly. 


\subsubsection{Vertical Dispersion}

As mentioned, one can correct the vertical dispersion directly with either skew quadrupoles in regions of horizontal clispersion or orbit bumps in the quadrupoles. ${ }^{[33]}$ Unfortunately, this is complicated because the skew quadrupoles and orbit bumps in the sextupoles also contribute to the betatron coupling. Thus, one has to either compensate the betatron coupling or use orbit bumps in regions without sextupoles; this will effectively limit the number of correctors one can use.

Regardless, the correction of the dispersion itself is relatively simple; the dispersion generated by random error's, much like a closed orbit, will primarily have harmonics near the vertical betatron tune. Thus, as when correcting a close orbit, only a few dispersion correctors are needed to cancel these dominant components, thersby significantly reducing the dispersion. We will consider two cases: (1) correctins the vertical emittance, i.e., the global effect of the dispersion, and (2) correcting the local dispersion at one location.

\section{Global Correction - Emittance correction}

The vertical emittance due to the dispersion is proportional to the average of $\overline{\mathcal{H}_{y}}$ in the bend magnets. For this estimate we will assume that this is equal to the average of $\overline{\mathcal{H}_{y}}$ around the ring. Using a few trigonometric identities, along with Eqs. (2.2.40), (2.2.42), and (2.2.57), we can express $\mathcal{H}_{y}$ as the squared absolute value of an integral over a complex exponential; this is very similar to the coupling coefficients $\left|Q_{ \pm}\right|^{2} / \sin ^{2} \pi \Delta \nu_{ \pm}$. Thus, the average of $\mathcal{H}_{y}$ in a ring with two correctors can be written

$$
\begin{aligned}
\overline{\mathcal{H}_{y}}=\frac{1}{4 \sin ^{2} \pi \nu_{y}}\left[\mid \int_{s}^{s+C} \sqrt{\beta_{y}(z) c^{\left.i l^{\prime} y(z) F(z) d z\right|^{2}}+a^{2}+b^{2}}\right. \\
+2 a \operatorname{Re} \int_{s}^{s+C^{\prime}} \sqrt{\beta_{y}(z) e^{i\left(2 \pi \nu_{y}-\psi_{y}(z)\right)} F(z) d z} \\
-2 b \operatorname{Im} \int_{s}^{s+C} \sqrt{\left.\beta_{y}(z) c^{i\left(2 \pi \nu_{y}-\psi_{y}(z)\right)} F(z) d z\right],}
\end{aligned}
$$

where the bar is used to denote an average around the ring and $a$ and $b$ are the strengths of the two correctors which are separated by $\pi / 2$ in phase and are arbjtrarily assumed to be located at $\iota^{\prime}=0$ and $\iota=-\pi / 2$. 
To minimize $\overline{\mathcal{H}_{y}}$, and thereby the emittance, we solve for the $a$ and $b$ which zero the first derivatives of $E q \cdot(2.2 .96)$. The solutions are

$$
\begin{aligned}
& a=-\operatorname{Re} \int_{s}^{s+C} \sqrt{\beta_{y}(z)} \epsilon^{i\left(2 \pi \nu_{y}-\psi_{y}(z)\right) F(z) d z} \\
& b=+\operatorname{Im} \int_{s} \sqrt{\beta_{y}(z)} e^{i\left(2 \pi \nu_{y}-\psi_{v}(z)\right)} F(z) d z,
\end{aligned}
$$

and these yield a residual dispersion of

$$
\overline{\mathcal{H}_{y}}=\frac{1}{4 \sin ^{2} \pi \nu_{y}}\left[\mid \int_{y}^{s+C} \sqrt{\beta_{y}(z)} e^{\left.i \nu^{\prime \prime} y(z) F(z) d z\right|^{2}}-a^{2}-b^{2}\right] .
$$

Now, we can solve for the expected value due to a distribution of random errors. Assuming that the errors $F_{i}$ are uniformly distributed in the ring, we find an expected value of

$$
\left\langle\overline{\mathcal{H}_{y}}\right\rangle=\frac{1}{6} \sum_{i}(F L)_{i}^{2} \beta_{y i},
$$

where $L_{i}$ is the length of the element at position $i$. This result should be compared with the uncorrected expected values, Eqs. (2.2.45) and (2.2.57). We see that using two correctors reduces the resiclual dispersion $\overline{\mathcal{H}_{y}}$ by a factor of $2 / 3 \sin ^{2} \pi \nu_{y}$.

Strictly, this result is only valid for the vertical dispersion due to uncorrelated errors, but because orbit correction effectively randomizes the orbit, we cari also apply the result to the dispersion due to a corrected orbit. Thus global dispersion correction, will reduce the expected values of the beam size and the emittance as

$$
\frac{\left\langle\sigma_{y}^{2}\right\rangle_{\text {local }}}{\beta_{y}}=\frac{2}{3} \sin ^{2} \pi \nu_{y} \frac{\left\langle\sigma_{y}^{2}\right\rangle_{0}}{\beta_{y}} \quad \text { and } \quad\left\langle\epsilon_{y}\right\rangle=\frac{2}{3} \sin ^{2} \pi \nu_{y}\left\langle\epsilon_{y}\right\rangle_{0}
$$

where the subscript 0 is used to denote the values before correction.

Notice that the correction cancels the resonant denominator. We will also find this cancellation when we estimate the effect of local correction. The resonant denominator occurs because the vertical clispersion is a periodic function that must close upon itself. When the dispersion is corrected, the boundary conditions are satisfied by the correctors; thus, the resonant denominator no longer appears. This is analogous to correcting the closed orbit or the coupling functions $Q_{+}$and $Q_{-}$, which are also periodic functions. 


\section{Local Correction}

Here, we use the two correctors to zero $\eta_{y}(s)$ and $\eta_{y}^{\prime}(s)$ at one location in the ring; this is equivalent to zeroing $\mathcal{H}_{y}(s)$. From Eqs. (2.2.40), (2.2.41), and (2.2.42), we find the required corrector strengths

$$
\begin{aligned}
& a=-\operatorname{Re} \int_{s}^{s+C} \sqrt{\beta_{y}(z)} e^{i\left(2 \pi \nu_{y}-\psi_{y}(z)\right)} F(z) d z \\
& b=+\operatorname{Im} \int_{s}^{s+C} \sqrt{\beta_{y}(z)} e^{i\left(2 \pi \nu_{y}-\psi_{y}(z)\right)} F(z) d z,
\end{aligned}
$$

where $s$ is the point that $\eta_{y}$ and $\eta_{y}^{\prime}$ are to be corrected to zero. Notice that these solutions are similar to the corrector solutions for global correction; they differ in that the solution for global correction is averaged while this is not.

Now, we need to know how this correction affects the global contribution, i.e., $\overline{\mathcal{H}_{y}}$. Here, we use Eq. (2.2.96) with the corrector strengths just calculated. Assuming random errors, we find

$$
\left\langle\overline{\mathcal{H}_{y}}\right\rangle=\frac{f_{\text {cor }}}{2} \sum_{i}(F L)_{i}^{2} \beta_{y i}
$$

which differs from the uncorrected result by a factor of $2 f_{\text {cor }} \sin ^{\prime \prime} n v_{y}$. Here, $f_{\text {cor }}$ is a function that depends upon the location of the correctors relative to the location that $\eta_{y}$ and $\eta_{y}^{\prime}$ are corrected. In particular,

$$
f_{\text {cor }} \approx\left(1-\frac{\Delta \psi_{y}}{2 \pi \nu_{y}}\right)^{2}+\left(\frac{\Delta \psi_{y}}{2 \pi \nu_{y}}\right)^{2}
$$

and $\Delta \psi$ is the phase difference from the correctors to the correction. This function varies between one and $1 / 2$, having a minimum when the correctors are separated from the correction point by half the ring and having a maximum when the correctors are immediately adjacent to the correction.

Applying this result, we sec that at most positions around the ring,

$$
\frac{\left\langle\sigma_{y}^{2}\right\rangle_{\text {local }}}{\beta_{y}}=2 f_{\text {cor }} \sin ^{2} \pi \nu_{y} \frac{\left\langle\sigma_{y}^{2}\right\rangle_{0}}{\beta_{y}} \quad\left\langle\epsilon_{y}\right\rangle=2 f_{\text {cor }} \sin ^{2} \pi \nu_{y}\left\langle\epsilon_{y}\right\rangle_{0}
$$

while at position $s:\left\langle\sigma_{y}^{2}(s)\right\rangle_{\text {local }} / \beta_{y} \sim 0$. Finally, notice that after local correction the emittance is between one and a half to three times larger than after global 
correction, Eq. (2.2.99); in the worst case, local correction actually increases the global contribution if the fractional tune is greater than 0.25 .

\section{Measurement}

Of course, to correct the vertical dispersion, one needs to measure it. If the BPMs are sufficiently accurate, one can measure the vertical dispersion directly by changing the beam energy. Alternately, if the BPMs are not sufficiently accurate, one can observe the effects of the vertical dispersion in the beam size. In this case, the vertical emittance is simply minimized with the correction elements. There are two problems with this approach: first, it is hard to decouple the local effect of the dispersion from the increase in the emittance, and second, the finite resolution of the beam size measurement will limit the convergence of the minimization; this will effectively limit the number of correctors that can be used in the minimization procedure.

\subsubsection{BETATRON COUPLING}

In this section, we will estimate the amount one can reduce the betatron coupling by directly correcting it with sliew quadrupoles or orbit bumps in the sextupoles. In Section 2.2.7, we calculated the reduction in $\overline{\mathcal{H}_{y}}$ after both global and local correction using just two correctors. The situation for the betatron coupling is similar, except we need four independent corrector's to correct both $Q_{+}$and $Q_{-}$.

To perform the calculation correctly, we would need to solve four coupled equations for the skew quadrupole strengths. Instead, we will make use of the analogy, noted in Section 2.2.5.1, between $Q_{ \pm}$and the vertical dispersion. This will allow us to use the results of the previous section. Of course, in treating the sum and difference resonance separately, we will neglect the effect of the cross term in Eq. (2.2.65). This is valid since, as was noted in Section 2.2.5, the cross term will tend to be small because of the rapidly oscillating phase. Furthermore, by treating $Q_{+}$and $Q_{-}$separately we assume that the correctors for $Q_{+}$do not affect $Q_{-}$and vise-versa. Obviously, this is not true of the individual skew quadrupoles, but linear combinations of the four skew quaclupoles can have this property.

\section{Global Correction - Emittance Correction}

To estimate the global corretion one can perform with four skew quadrupoles, we use the global coupling result of Scction 2.2.7. Thus, global correction will 
cancel the resonant denominators, reducing the expected values of the emittance and the beam size by $2 / 3 \sin ^{2} \pi \Delta \nu_{ \pm}$. Specifically, if four skew quadrupoles are used to correct the global coupling contribution, we use Eqs. (2.2.68) and (2.2.69) to find an expected residual due to uncorrelated error's of

$$
\begin{aligned}
& \frac{\left\langle\sigma_{y}^{2}\right\rangle_{\text {local }}}{\beta_{y}}=\frac{\epsilon_{x}}{12}\left[\sum_{\text {quad }}\left(K_{1} L\right)^{2} 4\left\langle\Theta^{2}\right\rangle \beta_{x} \beta_{y}+\sum_{\text {sext }}\left(K_{2} L\right)^{2}\left\langle y_{m}^{2}\right\rangle \beta_{x} \beta_{y}\right] \\
& \left\langle\epsilon_{y}\right\rangle=\frac{\epsilon_{x}}{12} \frac{\mathcal{T}_{x}}{\mathcal{J}_{y}}\left[\sum_{\text {quad }}\left(K_{1} L\right)^{2} 4\left\langle\Theta^{2}\right\rangle \beta_{x} \beta_{y}+\sum_{\text {sext }}\left(K_{2} L\right)^{2}\left\langle y_{m}^{2}\right\rangle \beta_{x} \beta_{y}\right],
\end{aligned}
$$

and we use Eq. (2.2.73) to find the residual due to a corrected closed orbit of

$$
\left\langle\epsilon_{y}\right\rangle=\left.\left.\sum_{\Delta \nu, \psi} \frac{\epsilon_{x}}{48} \frac{\mathcal{J}_{x}}{\mathcal{J}_{y}} \frac{\left\langle y_{c}^{2}\right\rangle}{\beta_{y}} \sum_{n_{c}}^{N_{\text {corr }}} \int_{n_{c}}^{n_{c}+1} \frac{d z_{i}}{\Delta s}\right|_{z_{i}} ^{n_{c}+1} d z K_{2}(z) \beta_{y}(z) \sqrt{\beta_{x}(z)} e^{i \psi}\right|^{2}
$$

where $\Delta s$ is the distance between correctors and the values of $\psi$ are given by Eq. (2.2.74).

\section{Local Correction}

To estimate the effect of local correction at position $s$, we could use the results derived in Section 2.2.7 as we did for the global correction. Alternately, we can observe the effect of local correction by examining Eq. (C.2.4). When the local coupling is corrected at location $s$, the first integral over $q_{ \pm}$is zero; this integral is equal to $Q_{ \pm}(s)$. The remaining term in Eq. (C.2.4) will cause an emittance contribution of

$$
\epsilon_{y}=\frac{\epsilon_{x}}{4} \frac{\mathcal{J}_{x}}{\mathcal{J}_{y}} \int_{s}^{s+C} \frac{d z_{i}}{C} \sum_{ \pm}\left|\int_{z_{1}}^{s+C^{\prime}} d z g \sqrt{\beta_{x} \beta_{y}} e^{i\left(\psi_{x} \pm \psi_{y}\right)}\right|^{2}
$$

where $s$ is the point of correction. Here, we have neglected the cross coupling terms. In addition, we have ignored the contribution from the correctors themselves. This is equivalent to assuming that the correctors are located just after point $s$ and thus they do not contribute to the integral. To include the correctors, we only need

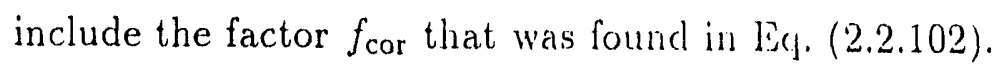


Now, we use Eq. (2.2.107) to find the emittance after correction of the local coupling with four skew quadrupoles. For random errors, we find

$$
\left\langle\epsilon_{y}\right\rangle=f_{\text {cor }} \frac{\epsilon_{x}}{4} \frac{\mathcal{J}_{x}}{\mathcal{J}_{y}}\left[\sum_{q u a d}\left(\Lambda_{1} L\right)^{2} 4\left\langle\Theta^{2}\right\rangle \beta_{x} \beta_{y}+\sum_{\text {sext }}\left(K_{2} L\right)^{2}\left\langle y_{m}^{2}\right\rangle \beta_{x} \beta_{y}\right]
$$

where $f_{\text {cor }}$ is given by $\mathrm{Eq},(2.2 .103)$. Notice that this result is equal to the estimate of local correction found in Eq. (2.2.102), namely, the correction changes the global coupling by $2 f_{\text {cor }} \sin ^{2} \pi \Delta \nu_{ \pm}$. Also notice that this is a factor of three larger than the result after global correction.

Finally, we estimate the effect of a corrected orbit after the local coupling has been corrected. Using Eçs. (2.2.107) and (2.2.73), we find

$$
\left\langle\epsilon_{y}\right\rangle=\left.\left.f_{\text {cor }} \sum_{\Delta \nu_{1} \psi^{\prime}} \frac{\epsilon_{x}}{16} \frac{\mathcal{J}_{x}}{\mathcal{J}_{y}} \frac{\left\langle y_{c}^{2}\right\rangle}{\beta_{y}} \sum_{n_{c}}^{N_{\text {cort }}} \int_{n_{c}}^{n_{c}+1} \frac{d z_{i}}{\Delta s}\right|_{z_{i}} ^{n_{c}+1} d z K_{2}^{\prime}(z) \beta_{y}(z) \sqrt{\beta_{x}(z)} e^{i \psi}\right|^{2},
$$

where $\Delta s$ is the distance between correctors and the values of $\psi$ are given above by $\mathrm{Eq} \cdot(2.2 .74)$.

\section{Measurement}

Finally, to perform these corrections, one needs to measure the coupling. Unfortunately, when operating a ring far from the coupling resonances, one cannot rely upon the standard techniclue of putting the ring on the difference resonance and then adjusting skew quadrupoles to make the two measured tunes equal. First, this technique does not correct the coupling due to the sum resonance. Second, the ring is perturbed when making the measurements and thus even the difference resonance will not be fully corrected when the ring is brought back to its nominal tunes.

Instead, the coupling can be nueasured by analyzing the coherent motion of a kicked beam. ${ }^{[7,15]}$ This measurement is convenient since one can measure the local value of the coupling all around the ring. Finally, as in the measurement of the dispersion, additional information can be obtained from measurements of the beam size at synchrotron light monitors or, in a damping ring, from the extracted beam. 
Table 7. $\epsilon_{y}$ from globally corrected vertical dispersion in the NDR1.

\begin{tabular}{|c|c|c|}
\hline Misalignment & Calc, $\epsilon_{y}[\mathrm{~m}$-radl $]$ & Simulated $\epsilon_{y}[\mathrm{~m}$-rad $]$ \\
\hline Random quad. $\Theta=0.5 \mathrm{mrad}$ & $0.72 \times 10^{-12}$ & $0.80 \pm 0.02 \times 10^{-12}$ \\
\hline Random sext. $y_{m}=150 \mu \mathrm{m}$ & $2.44 \times 10^{-12}$ & $2.35 \pm 0.04 \times 10^{-12}$ \\
\hline $\begin{array}{c}\text { Corrected closed orbit due to random } \\
\text { quad. } y_{m}=150 \mu \mathrm{m} \text { and BPM } y_{m}=150 \mu \mathrm{m}\end{array}$ & $0.50 \times 10^{-12}$ & $0.60 \pm 0.02 \times 10^{-12}$ \\
\hline
\end{tabular}

Table 8. $\epsilon_{y}$ from locally corrected coupling due to misalignments in the NDR1.

\begin{tabular}{|c|c|c|}
\hline Misalignment & Calc. $\epsilon_{y}[\mathrm{~m}$-rad] $]$ & Simulated $\epsilon_{y}[\mathrm{~m}$-rad] \\
\hline Random quad. $\Theta=0.5 \mathrm{mrad}$ & $1.52 \times 10^{-12}$ & $1.69 \pm 0.03 \times 10^{-12}$ \\
\hline Random sext. $y_{m}=150 \mu \mathrm{m}$ & $3.00 \times 10^{-12}$ & $3.36 \pm 0.06 \times 10^{-12}$ \\
\hline $\begin{array}{c}\text { Corrected closed orbit due to l'andom } \\
\text { quad. } y_{m}=150 \mu \mathrm{m} \text { and BPM } y_{m}=150 \mu \mathrm{m}\end{array}$ & $1.01 \times 10^{-12}$ & $1.7 \pm 0.2 \times 10^{-12}$ \\
\hline
\end{tabular}

\subsubsection{Simulations}

Simulations of the correction were performed in the NDR to verify these analytic estimates. First, the effect of correcting the global dispersion was simulated in the NDR1 ring. The correction was performed with two orbit bumps separated by roughly ninety degrees. The bumps were located in regions without sextupoles so there was no contribution to the betatron coupling. The results are listed in Table 7. Again, 1000 simulations were used to calculate the effect of the random misalignments and twenty simulations of a corrected closed orbit. Here, the estimates are found from Eq. (2.2.100) along with liq. (2.2.59) and the equations for random errors Eq. (2.2.45) or the equations for a corrected closed orbit, Eqs. (2.2.46), (2.2.50), and (2.2.55). Notice, by comparing with Table 4 , that the vertical emittance was decreased by roughly a factor of three. This is in excellent agreement with our estimate.

Next, the effect of correcting the local coupling was simulated. Four skew quadrupoles were used to completely uncouple the beam at the extraction point of the damping ring; two skew quads were located immediately adjacent to the extraction point while the other two were locatrd on the opposite side of the ring. The results are listed in Table 8. Again, 1000 simmlations were used to calculate the effect of the 
Table 9. $\epsilon_{y}$ from globally corrected coupling due to misalignments in the NDR1.

\begin{tabular}{|c|c|c|}
\hline Misalignment & Calc. $\epsilon_{y}[\mathrm{~m}$-rad] & Simulated $\epsilon_{y}[\mathrm{~m}$-rad $]$ \\
\hline Random quad. $\Theta=0.5 \mathrm{mrad}$ & $0.68 \times 10^{-12}$ & $0.71 \pm 0.08 \times 10^{-12}$ \\
\hline Random sext. $y_{m}=150 \mu \mathrm{m}$ & $1.33 \times 10^{-12}$ & $1.55 \pm 0.09 \times 10^{-12}$ \\
\hline $\begin{array}{c}\text { Corrected closed orbit due to landom } \\
\text { quad. } y_{m}=150 \mu \mathrm{m} \text { and BPM } y_{\mathrm{m}}=1.50 \mu \mathrm{m}\end{array}$ & $0.44 \times 10^{-12}$ & $0.7 \pm 0.1 \times 10^{-12}$ \\
\hline
\end{tabular}

random misalignments and twenty simulations of a corrected closed orbit. In this case, the calculated values are found using Eqs. (2.2.108) and (2.2.109) with a value $f_{\text {cor }}=0.75$ since two corrector's are adjacent to the point of correction $\left(f_{\text {cor }}=1.0\right)$ and two are halfway around to ring lrom the point of correction $\left(f_{\text {cor }}=0.5\right)$. Notice that the vertical emittance due to the errors is roughly a factor of four smaller than before the correction. Again, the simulated results agree well with the calculated values.

Finally, the effect of correcting the global coupling was simulated. This time the four skew quadrupoles were used to minimize the vertical emittance at the extraction point of the damping ring. The results are listed in Table 9. Here, only 100 simulations were used to calculale the effect of the random misalignments and twenty simulations of a corrected closed orbit; the global correction simulations are computation intensive. In this case, the calculated values are found using Eqs. (2.2.105) and (2.2.106). Notice, by comparing with Table 5, that now the vertical emittance due to the errors is roughly a factor of nine smaller than before the correction and, again, the calculated estimates agree sell with results of the simulations.

\subsubsection{Distributions and Tol'rances}

In Sections $2.2 .4,2.2 .5,2.2,6$, and 2.2 .7 , we have calculated the expected values of the vertical emittance and the beam size. Naively, one could simply invert these equations to solve for aligmment tolerances. But, when specifying tolerances, one should include a "conficlence level" (CL); this is the probability that, given the specified tolerances, any specific machine will be less than the design limit. Typically, one wants to specify a large ( $L$ so that there is a small probability of exceeding the design limit. In this section. we will calculate the location of the 95\% CL as a function of the expected values calculated previously. 


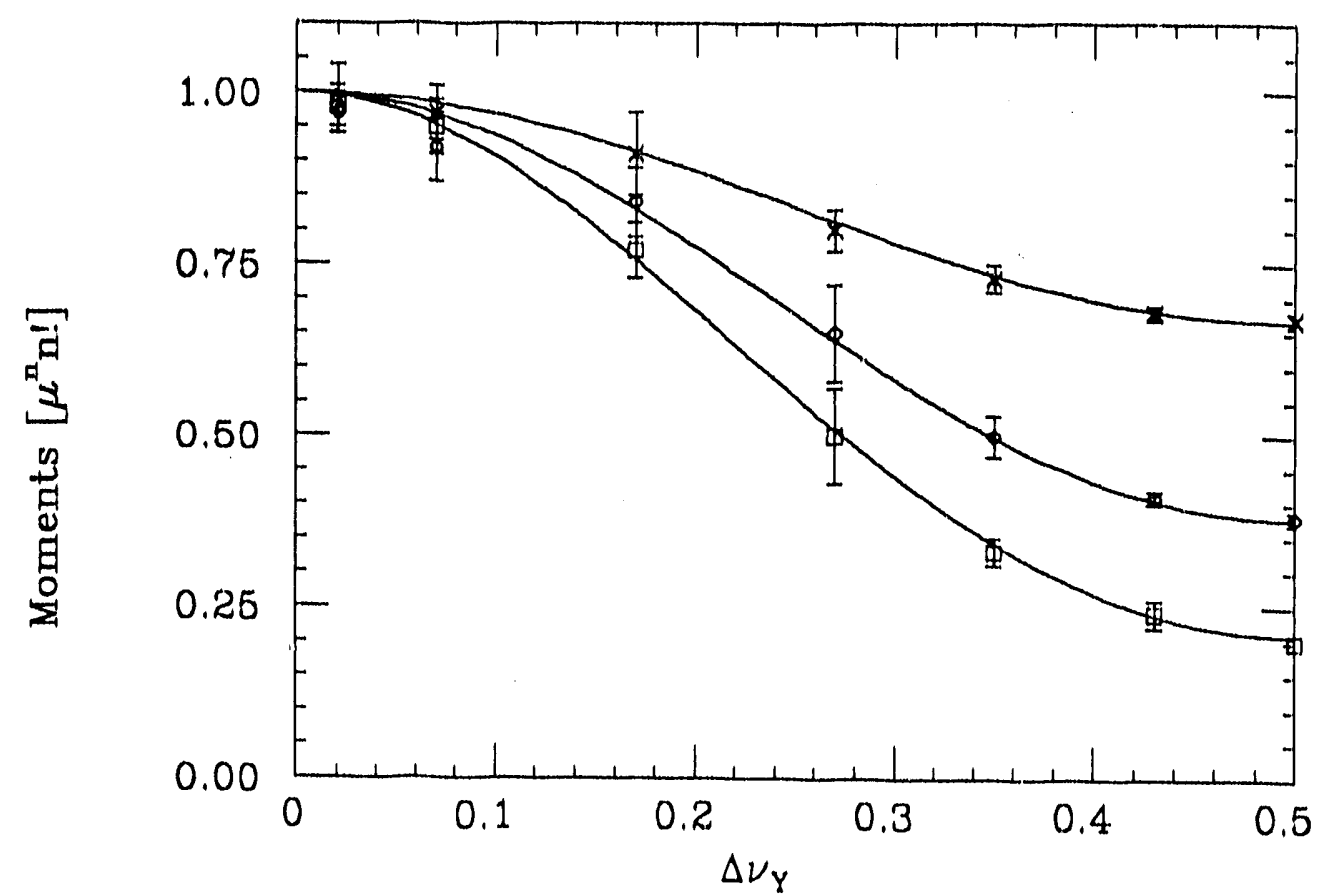

Fig. 8. Second, third, and fourth normalized moments of the distribution for $\epsilon_{y}$ from dispersion due to random error's versus the fractional tune; the second moment is the largest and the fourth moment is the smallest. The data points are found from simulations.

Calculating the CL requires a detailed knowledge of the distribution of the values of the emittance and the bean size in an ensemble of machines. It has been shown that the mean square amplitude of the normalized orbit due to random errors with gaussian distributions should have an exponential distribution function. ${ }^{[7]}$ Since the equations for the closed orbit are similar to those of the dispersion function and the betatron coupling, the same result applies to the amplitudes of $\mathcal{H}_{y}(s)$ and $\left|Q_{ \pm}(s)\right|^{2}$.

Here, we will consider the effect of averaging $\mathcal{H}_{y}(s)$ and $\left|Q_{ \pm}(s)\right|^{2}$ over $s$; the vertical emittance is equal to the average of these functions in the bending magnets. We will first discuss the distribution of the values of the emittance arising from vertical dispersion and betatron coupling due to random errors. Then, we will discuss the distribution of the values of the local contribution to the vertical beam size. Finally, note that, although the discussion is limited to the effect of random misalignment errors, the effect of a corrected closed orbit is similar.

\subsubsection{EMITtTANCE DUE TO VERTICAL DISPERSION}

The distribution function for the values of the vertical emittance due to random 
errors is a very complicated function. Thus, we will derive an approximate form that can be integrated to solve for the location of the $95 \% \mathrm{CL}$. We do this by solving for the moments of the distribution of emiltances. The vertical emittance is given by Eq. (2.2.58), Assuming identical bending magnets, we can express this in the same form as $\mathrm{Eq} .(2.2 .96)$ :

$$
\epsilon_{y}=\frac{\mathcal{T}_{\epsilon} \sigma_{c}^{2}}{4 \sin ^{2} \pi \nu_{y}}\left|\int_{g}^{s+C} \sqrt{\beta_{y}(z) e^{i \psi_{v}(z)} F(z) d z}\right|^{2} .
$$

where $F$ is defined in $\mathrm{Eq},(2.2 .40)$.

Now, we solve for the moments assuming random errors with gaussian distributions. This yields

$$
\begin{aligned}
& \left\langle\epsilon_{y}\right\rangle=\mu \\
& \left\langle\epsilon_{y}^{2}\right\rangle=2 \mu^{2}\left(1-\frac{1}{3} \sin ^{2} \pi \nu_{y}\right) \\
& \left\langle\epsilon_{y}^{3}\right\rangle=6 \mu^{3}\left(1-\frac{2}{3} \sin ^{2} \pi \nu_{y}+\frac{2}{45} \sin ^{4} \pi \nu_{y}\right) \\
& \left\langle\epsilon_{y}^{4}\right\rangle \approx 24 \mu^{4}\left(1-\sin ^{2} \pi \nu_{y}+\frac{1}{3} \sin ^{4} \pi \nu_{y}-\frac{2}{15} \sin ^{6} \pi \nu_{y}\right),
\end{aligned}
$$

where $\mu$ is the expected valuc of the emittance calculated in Section 2.2.4. The first three moments were calculated from Eq,$(2.2 .110)$, while the fourth monent was fit to data from simulations. These are shown in Figure 8 where the second, third, and fourth moments, nomalized by $n ! \mu^{n}$, are plotted.

Notice that the moments only depend upon the first moment $\mu$ and the fractional vertical tune. When the vertical ture is close to an integer, the moments have the form $\mu_{n}=n ! \mu^{n}$. These are Ho moments of an exponential distribution as noted in Ref. 71. As the fractional tune increases, the moments decrease, implying that the probability of large emitianco values is decreased.

We could attempt to construct a distribution directly from these moments, but, instead, we simply notice that these moments are close to those of a modified $\chi$ squared distribution where the number of degrees of freedom is a function of $\sin ^{2} \pi \nu_{y}$. In particular, the distribution density can be approximated by

$$
g\left(c_{y}\right) \approx \frac{n}{2 \mu} \frac{e^{-\epsilon_{y} n / 2 \mu}}{\Gamma\left(\frac{n}{2}\right)}\left(\frac{c_{y} n}{2 \mu}\right)^{\frac{n}{2}-1},
$$

where $\mu$ is the expected value of the enittance and $n$ is the number of degrees of 
freedom which depends upon $\sin ^{2} \pi v_{y}:$

$$
\frac{n}{2}=\frac{1}{1-\frac{2}{3} \sin ^{2} \pi \nu_{y}} .
$$

With these definitions, this distribution has the same first and second moments as the value of the vertical emittance, $\mathrm{Ec}_{1} \cdot(2.2 .111)$. Furthermore, when the tune is integral, Eq. (2.2.112) is correctly equal to the density of an exponential distribution, and, when the fractional tune increases to 0.5 , the third and fourth moments of Eq. (2.2.112) are within $2 \%$ and $8 \%$ of the moments of the value of the vertical emittance.

These distributions are illustrated in Figure 9 where the distribution density of the vertical emittance, arising from random errors, has been plotted for three different tunes. All of the histograms are generated from 1000 simulations of $150 \mu \mathrm{m}$ vertical sextupole misalignments in the NDR. In Figure $9\left(\right.$ a), the tune is $\nu_{y}=3.07$, while in Figures $9(\mathrm{~b})$ and $9(\mathrm{c})$ the tunes aro $\nu_{y}=3.275$ and $\nu_{y}=3.43$. In addition, the approximate distribution density of $\mathrm{Eq} .(2.2 .112)$ is plotted for each of these three cases. One can see that there is fairly good agreement between the simulations and the approximation.

Now, we need to calculate the clistribution after correction of the vertical dispersion. After global correction, the expected value of the emittance is given by Eq. (2.2.100). In addition, the second moment of the distribution can be found from Eq. (2.2.96). It is

$$
\left\langle\epsilon_{y}^{2}\right\rangle=2 \mu^{2}\left(\frac{7}{5}\right)
$$

Notice that this second moment is independent of the tune. Thus, we would expect the $95 \%$ CL to only be weakly dependent upon the fractional tune. To approximate this distribution, we simply choose $n$ to equate the second moment of $\mathrm{Eq},(2.2 .112)$ with this second moment; this occurs when $n=5.0$.

The distribution density of the value of the vertical emittance after global correction is illustrated in Figure 9(d). The data was found from 1000 simulations of random sextupole misalignments in the NDR 1 and the approximate distribution is found from $\mathrm{Eq}$. (2.2.112) where $n=5.0$. Here, our approximation does not accurately reproduce the distribution clensity for emittance values less than $2\left\langle\epsilon_{y}\right\rangle$, but it does describe the tail of the distribution well; this is ultimately what we need to know to calculate the location of the $95 \%$ (IL. 
Chapter 2.2.8.1
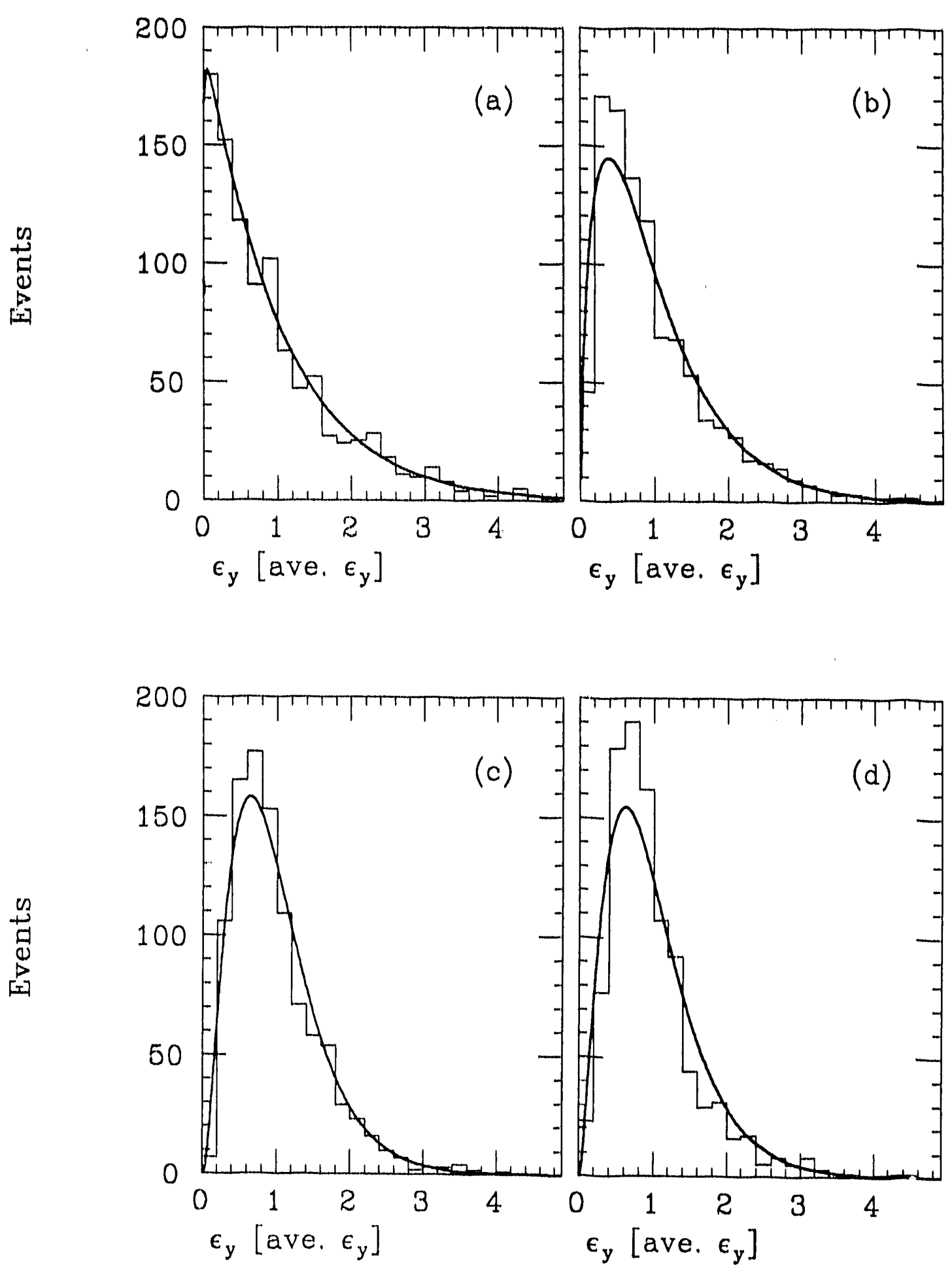

Fig. 9. Events versus cy clue to the vertical dispersion in the NDR1 lattice. Histograms are calculated from 1000 simulations of random vertical sextupole misalignments wit/ ling tunes of: (a) $\nu_{y}=3.07$, (b) $\nu_{y}=3.275$, (c) $\nu_{y}=3.43$, and (d) $\nu_{y}=3.275$ after global correction. The curves are calculated from Eq. (2.2.112). 


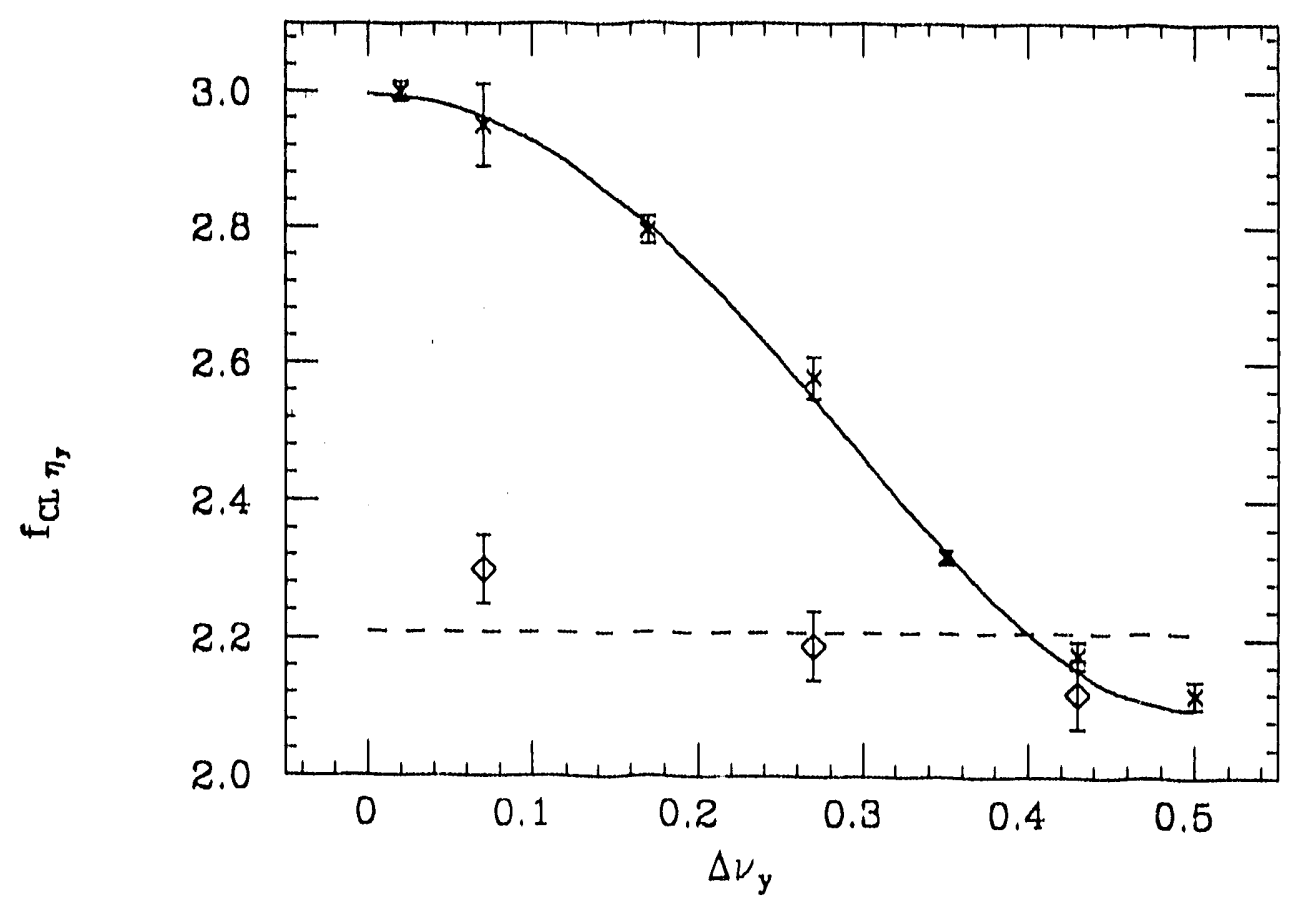

Fig. 10. $95 \%$ confidence level fol $\epsilon_{y}$ due to dispersion versus the fractional tune.

At this point, we can calculate the location of the $95 \%$ CL for the distributions. This found by integrating the distribution density

$$
\int_{0}^{f_{C L}\left\langle\left\langle\left(c_{y}\right)\right.\right.} g\left(c_{y}\right) d c_{y}=0.95,
$$

where $f_{\mathrm{CL}}$ is the location of the $9.5 \% \mathrm{CL}$ in units of the expected vertical emittance. The results are plotted in Figure 10 as a function of the fractional vertical tune $\Delta \nu_{y}$. The solid curve is calculated from E $\mathrm{q}_{1}$. (2.2.112), while the simulation results are plotted as crosses. One can sec that there is very close agreement between the simulation and the approximation results.

In addition, the value of $f_{\mathrm{CL}}$ after global correction of the dispersion has also

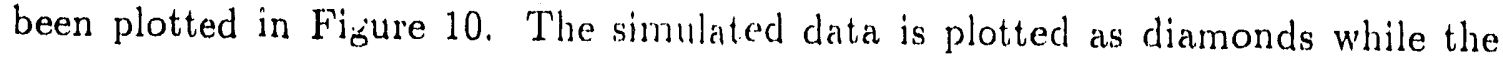
dashed line is our approximation. Although the approximation for the correction does not agree well with the simulated results, as expected, $f_{\mathrm{CL}}$ is only weakly dependent upon the fractional rune and it is usually much less than the $f_{\mathrm{CL}}$ of the uncorrected cases.

Finally, it is important to note the following: first, the curves for $f_{C L}$ are universal. The only dependence comes from the fractional vertical tune. The value of $f_{\mathrm{CL}}$ 
is independent of the type of errors, the lattice type, and the integral portion of the tune. The data in Figure 10 has been compared with simulations run on the ALS: a Triple Bend Achromat lattice with an integral tune of 8 , and a future damping ring design: ${ }^{[80]}$ a FODO lattice with an integral tune of 11 . In both cases, excellent agreement was found with the curve in Figure 10.

Second, our calculations have assumed that the errors are random with gaussian distributions. A more realistic error distribution is a gaussian distribution where the tails are cutoff at $\pm 2 \sigma$; it is cloubtiful that large alignment errors, values that are many $\sigma$, would go undetected. This will reduce $f_{\mathrm{CL}}$ even further, making Figure 10 a ronservative estimate of $f_{\mathrm{CL}}$.

And lastly, notice that there are two advantages of increasing the fractional tune towards a half-integer: the expected value of the emittance decreases, and the probability of large deviations above this expected value also decreases.

\subsubsection{Emittance due to Betatron Coupling}

Now, we an use the results of the previous section to calculate the distribution of the value of the vertical cmittance arising from betatron coupling. Ignoring the cross term in $\mathrm{Eq}$. $(2.2 .65)$, the enittance is the sum of the two quantities $\overline{\left.Q_{ \pm}\right|^{2}}$. As noted earlier, these two values have the same form as $\overline{\mathcal{H}_{y}}$ and thus they should each have approximate distributions given by Eq. (2.2.112). Furthermore, if $\overline{\left|Q_{+}\right|^{2}}$ and $\overline{\left|Q_{-}\right|^{2}}$ are mutually indepenclent, then the distribution of their sum is just the convolution of the two inclividual distributions.

Since we have assumed that the errors have gaussian distributions, $Q_{+}$and $Q_{-}$ will be independent if ${ }^{[133]}$

$$
\begin{aligned}
& \int_{s}^{s+C} d z\left\langle k^{2}(z)\right\rangle \beta_{x} \beta_{y} e^{i 2 \psi^{\prime} x}=0 \\
& \int_{s}^{s+C^{\prime}} d z\left\langle k^{2}(z)\right\rangle \beta_{x} \beta_{y} e^{i 2 \psi_{y}}=0,
\end{aligned}
$$

where $\tilde{k}=K_{2} y-\widetilde{K}_{1}$. Both of these conditions will be (approximately) satisfied if there are many errors in a betatron period, $N \gg \nu_{x, y}$, and if the tunes are large, $\nu_{r, y} \gg 1$ : this is typical of high tume (low emittance) rings. 
Convolving the two individual distributions for $\overline{\left|Q_{+}\right|^{2}}$ and $\overline{\left|Q_{-}\right|^{2}}$, we find an approximate distribution for the value of the vertical emittance:

$$
\begin{aligned}
g\left(\epsilon_{y}\right) \approx & \left(\frac{n_{+}}{2 \mu_{+}}\right)^{\frac{n_{t}}{2}}\left(\frac{n_{-}}{2 \mu_{-}}\right)^{\frac{n_{-}}{2}} \frac{e^{-\epsilon_{y} n_{-} / 2 \mu_{-}}}{\Gamma\left(\frac{n_{+}}{2}\right) \Gamma\left(\frac{n_{-}}{2}\right)} \\
& \times \int_{0}^{\epsilon_{y}} d x e^{-x\left(\frac{n_{+}}{2 \mu_{+}}-\frac{n_{-}}{2 \mu_{-}}\right)} x^{\frac{n_{t}}{2}-1}\left(\epsilon_{y}-x\right)^{\frac{n_{-}-1}{2}},
\end{aligned}
$$

where $n_{+}$and $n_{-}$are

$$
\frac{n_{ \pm}}{2}=\frac{1}{1-\frac{2}{3} \sin ^{2} \pi \Delta \nu_{ \pm}}
$$

and $\mu_{ \pm}$are the expected values of the contributions from the sum and difference resonances; these were found in Section 2.2.5. Although the integral in Eq. (2.2.117) can be expressed in terms of the degenerate hypergeometric function, sometimes called Kummer's function, there is no simple evaluation and is thus left as is.

The distribution of the emittances is illustrated in Figure 11 where the distribution density is plotted for two sets of tunes. In Figure 11(a) the tunes are $\nu_{x}=8.375$ and $\nu_{y}=3.275$ so that $\Delta \nu_{+}=0.35$ and $\Delta \nu_{-}=0.10$, while in Figure 11(b) the tunes are $\nu_{x}=8.425$ and $\nu_{y}=2.925$ so that $\Delta y_{+}=0.35$ and $\Delta \nu_{-}=0.50$. As before the histograms are found from 1000 simulations of random sextupole errors and the curves are calculated from Eq. (2.2.117). Again, there is very good agreement between the simulations and the approximation.

Now, we can calculate the location of the $95 \%$ CL which, in the case of the betatron coupling, is a function of both $\Delta \nu_{+}$and $\Delta \nu_{-}$. This is illustrated in Figure 12 where $f_{\mathrm{CL}}$ is plotted as a function of $\Delta \nu_{-}$, for $\Delta \nu_{+}=0.35$. The crosses are the results of simulations and the solid line is calculated from Eq. (2.2.117). In addition, the $f_{C L}$, found from 100 simulations of global correction, is plotted for three different tunes; this data is plotted as diamonds while the estimated value, found using the approximation of Eq. (2.2.114), is plotted as a dashed line.

One can see that there is very good agreement between the simulated results and the approximation when $\Delta \nu_{-}$is small, but there is a significant discrepancy as

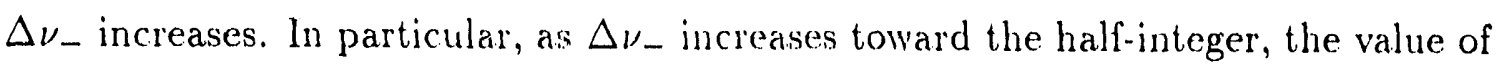
$f_{\mathrm{CL}}$ appears to depend upon the horizontal and vertical tunes in addition to $\Delta \nu_{+}$ and $\Delta \nu_{-}$. For example, when the tunes ale $\nu_{r}=8.575$ and $\nu_{y}=3.075\left(\Delta_{y_{+}}=0.35\right.$ 


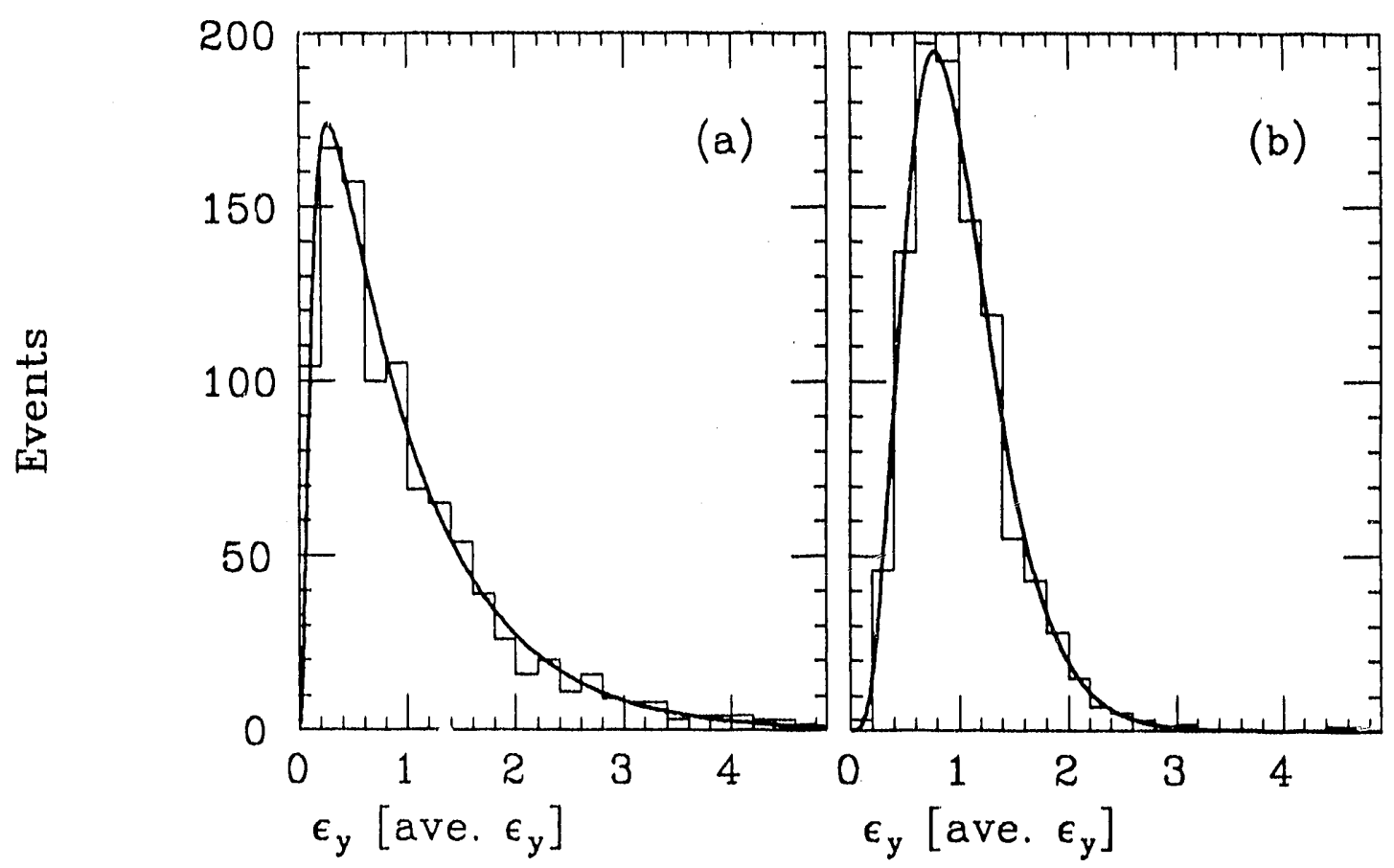

Fig. 11. Events versus $\epsilon_{y}$ due to the linear coupling in the NDR1 lattice. Histograms are calculated from 1000 simulations of random vertical sextupole misalignments for tunes of: (a) $\Delta \nu_{+}=0.35$ and $\Delta \nu_{-}=0.10$, and (b) $\Delta y_{+}=0.35$ and $\Delta y_{-}=0.50$. The curves are calculated with Eq. (2.2.117).

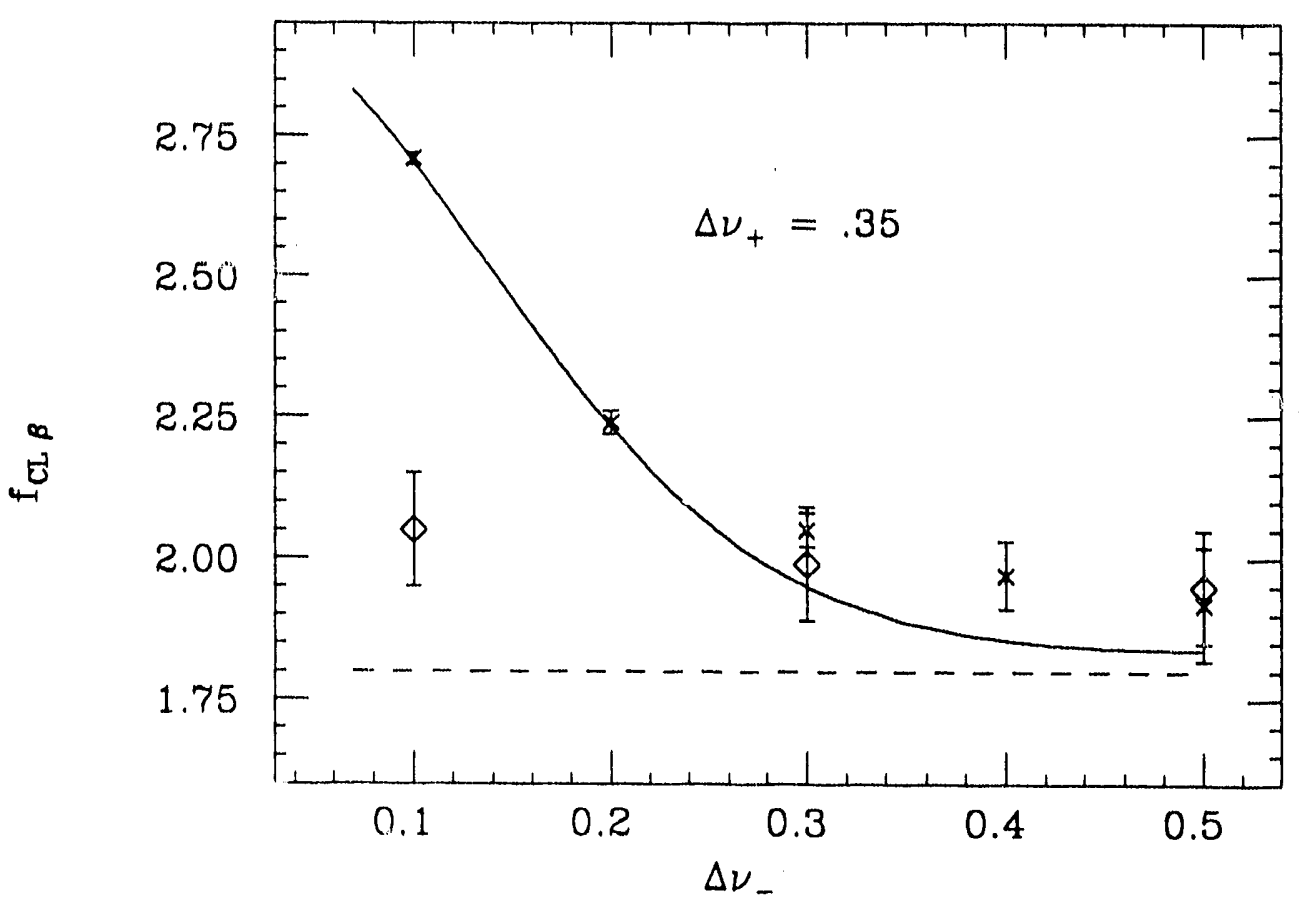

Fig. 12. $95 \%$ conficlence level for $\epsilon_{y}$ due to betatron coupling versus the distance from the difference coupling resonance for $\Delta \nu_{+}=.35$. 
and $\left.\Delta \nu_{-}=0.50\right), f_{C L}$ equals 2.05. In contrast, when the tunes are $\nu_{x}=8.425$ and $\nu_{y}=2.925\left(\Delta \nu_{+}=0.35\right.$ and $\left.\Delta \nu_{-}=0.50\right), f_{C L}$ equals 1.86. Thus, there is a substantial difference in $f_{C L}$ even though $\Delta \nu_{ \pm}$are the same in the two cases. This difference could be explained by the cross term in Eqs. (2.2.65) and (2.2.70) which depends upon $\sin 2 \pi \nu_{x}$ along with $\sin \pi \Delta \nu_{ \pm}$.

Finally, again notice the following: (1) the curves in Figure 12 are universal in that all rings will have similar values of $f_{\mathrm{CL}},(2)$ the values of $f_{\mathrm{CL}}$ in Figure 12 are conservative since the actual disiributions of errors will probably not have large value tails, and (3) there are two advantages of keeping $\Delta \nu_{-}$and $\Delta \nu_{+}$large: the expected value of the emittance decreases and the probability of large deviations above this expected value also decreases.

\subsubsection{LOCAL BEAM SIZE}

Now, we can calculate the distribution of the value of the beam size arising from the local coupling effects. These are simpler than the distributions of the emittances since the contribution depends upon the local value, not the average value, of the coupling. In the case of the dispersion, the beam size $\sigma_{y}^{2} / \beta_{y}$ depends upon $\eta_{y}^{2}(s) / \beta_{y}$. As stated, this will have an exponential distribution similar to the closed orbit. ${ }^{[7]}$ Thus, the value of the projected emittance increase due to dispersion will have an exponential distribution with a $95 \%$ C.L located at $3.00\left\langle\sigma_{y}^{2}\right\rangle / \beta_{y}$; this is equal to the distribution of $\mathrm{Eq} \cdot(2.2 .112)$ where $n=2$ instead of the value specified in $\mathrm{Eq} .(2.2 .113)$.

Similarly, the beam size due to local effect of the betatron coupling depends upon both $\left|Q_{-}^{2}(s)\right|$ and $\left|Q_{+}^{2}(s)\right|$ which also have exponential distributions. Thus, the resulting distribution can be found from Eq. (2.2.117) where $n_{ \pm}=2$ instead of the values specified in $\mathrm{Ec}_{1} .(2.2 .118)$. In this case, we can evaluate the integral in Eq. (2.2.117), finding

$$
g\left(\left\langle\sigma_{y}^{2}\right\rangle / \beta_{y}\right)=\frac{\epsilon^{-\left\langle\sigma_{y}^{2}\right\rangle / \beta_{y} \mu_{+}}-\epsilon^{-\left\langle\sigma_{y}^{2}\right\rangle / \beta_{y} \mu_{-}}}{\mu_{+}-\mu_{-}},
$$

where $\mu_{ \pm}$are the expected contributions for the sum and difference resonances, found in Section 2.2.5. Now, the location of the $95 \% \mathrm{CL}$ can be calculated directly from this "bi-exponential" distribution. It ranges from $f_{\mathrm{CL}}=3.00$, when $\mu_{+} \gg \mu_{-}$ or $\mu_{-} \gg \mu_{+}$, to $f_{\mathrm{CL}}=2.37$, when $\mu_{+}=\mu_{-}$. 


\subsubsection{TOlerances}

Finally, one can use the results of this section to calculate tolerances. We have found that the $95 \%$ CL occurs at a value between roughly two and three times the expected emittance. To calculate alignment tolerances with a $95 \% \mathrm{CL}$, we simply solve for tolerances that yield expected values that are a factor $f_{\mathrm{CL}}$ smaller than the design values.

For example, if we wish to limit the vertical emittance due to sextupole misalignments, we can use Eqs. (2.2.45) and (2.2.69) along with the appropriate values of $f_{\mathrm{CL}}$ to solve for the $95 \%$ CL emittance:

$$
\begin{aligned}
\epsilon_{y 95 \%}= & {\left[\frac{J_{\epsilon} \sigma_{\epsilon}^{2}}{4 \sin ^{2} \pi \nu_{y}}\left(\sum_{s \epsilon x t}\left(\kappa_{2} L\right)^{2} \eta_{x}^{2} \beta_{y}\right) f_{\mathrm{CL} \eta_{y}}\right.} \\
& \left.+\frac{\epsilon_{x}}{4} \frac{\left(1-\cos 2 \pi \nu_{x} \cos 2 \pi \nu_{y}\right)}{\left(\cos 2 \pi \nu_{x}-\cos 2 \pi \nu_{y}\right)^{2}} \frac{\alpha_{x}}{\alpha_{y}}\left(\sum_{s e x t}\left(K_{2} L\right)^{2} \beta_{x} \beta_{y}\right) f_{\mathrm{CL} \beta}\right] y_{m}^{2},
\end{aligned}
$$

where $f_{\mathrm{CL} \eta_{\nu}}$ can be found from Figure 10 and $f_{\mathrm{CL} \beta}$ can be found from Figure 12. It is trivial to invert this to solve for the desired alignment tolerance.

Actually, the factors $f_{\mathrm{CL}}$ were calculated for the dispersive contribution and coupling contribution individually. Strictly, to calculate the $f_{\mathrm{CL}}$ for the sum of the two contributions requires convolving both distributions. Fortunately, one usually finds that either the dispersive or the coupling contribution dominates and thus the separate values $f_{\mathrm{CL}}$ can be used accurately. However, if both contributions are of equal magnitude, this method will result in tolerances that are slightly too severe.

\subsubsection{Summary}

In this chapter, Secion 2.2. we have discussed the dominant low current contributions to the vertical emittance and beam size in $e^{-t} / \epsilon^{-}$storage rings, namely, the vertical dispersion and the betatron coupling. In addition, we have calculated the synchro-betatron coupling and presented a corrected derivation for the emittance contribution from the opening angle of the synchrotron radiation. These are negligible contributions in the current clesigns, although, the later effect does specify a lower bound on the vertical miltance and may be an important linitation in the future. 
The vertical dispersion and the betatron coupling are generated by both magnet alignment errors and a non-zero beam trajectory. We have calculated the expected contributions to the vertical emittance and the vertical beam size due to random misalignments of the magnets and a corrected closed orbit. In addition, we have carefully separated the contributions to the vertical rattifnce and the beam size since local coupling effects can increase the beam size ithout increasing the emittance. This is important since the emittance is the relevaht quantity in some instances while the beam size is in others.

We have also estimated the effectiveness of simple correction techniques in reducing both the vertical emittance and the beam size. In particular, we used one pair of correctors to reduce the vertical dispersion and four skew quadrupoles to reduce the betatron coupling. In general, the correctors reduce the emittance by cancelling the resonant denominators found in the expressions for the emittance due to dispersion or betatron coupling. Of course, two clispersion correctors or four skew quadrupoles cannot be used to zero the respective enittance contributions anymore than two dipole correctors can be used to zero the closed orbit at all locations around a ring.

Finally, we have calculated alignment tolerances to limit the vertical emittance and beam size from the vertical dispersion and the betatron coupling. In particular, we have calculated approximate distribution functions for the values of the emittance and beam size in an ensemble of machines. From these distributions, we found tol rances that limit the vertical enittance and beam size with a $95 \%$ confidence level. In general, these are a factor of $\sqrt{2}$ to $\sqrt{3}$ more severe than tolerances simply calculated from the expected values of the emittance and beam size. It is thought that this analysis could greatly simplify the calculation of alignment tolerances to limit the vertical emittance and beam size, thereby reducing the need for extensive simulation.

\subsubsection{Application to NLC Damping Ring}

At this point, we can apply the results of this section to the NLC damping ring design. Of course, the NLC ring design is not finalized and thus these will be preliminary tolerances. The ring consists of two familics of sextupoles and twelve families of quadrupoles (including the combined function bending magnets). The effect of random sextupole misalignments and quadrupole rotations is listed in Table 10 where we have calculated the normalized emittance in m-rad as a function of the error 
Table 10. Vertical emittance budget for NLC damping ring.

\begin{tabular}{|c|c|c|c|}
\hline & Dispersion $\left\langle\gamma \epsilon_{y}\right\rangle$ & $\beta$ coupling $\left\langle\gamma \epsilon_{y}\right\rangle$ & $\gamma \epsilon_{y} 95 \% \mathrm{CL}$ \\
\hline SF \& SD (bends) & $0.84 y_{m}^{2}$ & $0.52 y_{m}^{2}$ & $2.80 y_{m}^{2}$ \\
\hline SF \& SD (wigglers) & $1.6 y_{m}^{2}$ & $0.13 y_{m}^{2}$ & $3.75 y_{m}^{2}$ \\
\hline QF (bends) & $3.2 \times 10^{-3} \Theta_{q}^{2}$ & $1.6 \times 10^{-3} \Theta_{q}^{2}$ & $9.8 \times 10^{-3} \Theta_{q}^{2}$ \\
\hline QF (wigglers) & $6.2 \times 10^{-3} \Theta_{q}^{2}$ & $0.5 \times 10^{-3} \Theta_{q}^{2}$ & $14.5 \times 10^{-3} \Theta_{q}^{2}$ \\
\hline other quads (bends) & $0.6 \times 10^{-3} \Theta_{q}^{2}$ & $1.6 \times 10^{-3} \Theta_{q}^{2}$ & $4.2 \times 10^{-3} \Theta_{q}^{2}$ \\
\hline other quads (wigglers) & $1.1 \times 10^{-3} \Theta_{q}^{2}$ & $0.5 \times 10^{-3} \Theta_{q}^{2}$ & $3.3 \times 10^{-3} \Theta_{q}^{2}$ \\
\hline
\end{tabular}

Table 11. Tolerances for the NLC damping ring.

\begin{tabular}{|c|c|c|}
\hline & Tolerances & $\gamma \epsilon_{y} 05 \% \mathrm{CL}$ \\
\hline $\mathrm{SF} \& \mathrm{SD}$ & $y_{m}=75 \mu \mathrm{m}$ & $1.7 \times 10^{-8} \mathrm{~m}-\mathrm{rad}$ \\
\hline QF & $\Theta_{y}=0.25 \mathrm{mrad}$ & $0.1 \times 10^{-8} \mathrm{~m}-\mathrm{rad}$ \\
\hline Other Quads & $\Theta_{y}=0.50 \mathrm{mrad}$ & $0.1 \times 10^{-8} \mathrm{~m}-\mathrm{rad}$ \\
\hline BPMs & $y_{m}=100 \mu \mathrm{m}$ & $0.4 \times 10^{-8} \mathrm{~m}-\mathrm{rad}$ \\
\hline$\eta_{y}$ wigglers & $\eta_{y} \mathrm{rms}=1 \mathrm{~mm}$ & $0.2 \times 10^{-8} \mathrm{~m}-\mathrm{rad}$ \\
\hline
\end{tabular}

strength assuming global correction of both the dispersion and the coupling; $y_{m}$ is the rms vertical alignment in meters and $\Theta_{q}$ is the rms rotational alignment in radians. The contributions are divided into dispersive and coupling effects due to both the bending magnets and lhe wigglers. Finally, the table also lists the maximum emittance, with a $95 \%$ confidence lovel, due to both the dispersion and the betatron coupling; we used $f_{C L} \eta_{y}=2.2$ and $f_{C L \beta}=1.8$.

Notice that, after correction, the dispersive contributions are larger than the betatron coupling contributions. Furthermore, the effect of vertical dispersion in the wigglers is severe; the coupling in the wigglers is small because the horizontal dispersion is small. We will estimate tolerances assuming that the dispersion is locally corrected in the wiggler's to $1 \mathrm{~mm} \mathrm{rms}$. In addition, we assume that the rms BPM and quadrupole misalignments are comparable to the sextupole alignment and thus the effects of the closed orbit are small.

The resulting tolerances are listed in Table 11. Obviously, the sextupole align- 
ment tolerances are the most severe at $75 \mu \mathrm{m}$. The sum of the emittance contributions is $\gamma \epsilon_{y} \leq 2.5 \times 10^{-8} \mathrm{~m}-\mathrm{rad}$ with a confidence level of $95 \%$. The expected value of the vertical emittance is roughly a factor of two smaller. 


\section{CHAPTER $\quad 2.3$ \\ COLLECTIVE LIMITATIONS}

In this chapter, we will discuss current dependent phenomena that could limit the vertical emittance. The primary issues are: intrabeam scattering, ion trapping, direct effects of the bearn's space charge field, and the interaction of the space charge field with the environment, namely, the sucuum pipe. This last category will be separated into both static effects and instabilities. Finally, we will also discuss the effect of beam-gas scattering and lifetime limitations.

\subsubsection{Intrabeam Scattering}

Intrabeam scattering is the result of multiple small angle Coulomb collisions between particles in the beam leading to diffusion. In addition, there are relatively infrequent large angle Coulomb collisions where a particle can gain momenta that exceed the machine aperture. This causes particle loss and is referred to as the Touschek effect; it will be discussed in Section 2.3.7.

In $e^{+} / e^{-}$storage rings, intrabeam scattering increases the equilibrium emittances until the additional diffusion is countered by the radiation damping. Detailed theories of intrabeam scattering have been developed in Refs. 17, 72, and 79. These theories are complex and, in general, require numerical evaluation. Here, we will describe the basic physics in $e^{+} / e^{-}$rings using some analytic results from Ref. 79. Then, we discuss the limitations that intrabeam scattering imposes on the vertical emittance and it's effect on the vertical damping rate. Finally, we discuss the scaling of the intrabeam phenomena with the storage ring energy.

\subsubsection{THEORY}

Intrabeam scattering will redistribute the beam momenta in an approach to "thermal" equilibrium. In a reference frame co-moving with the particle beam, the beam usually has an anisotropic momentum distribution. Assuming an ultrarelativistic beam, the beam frame momentum spreads are given by

$$
\left\langle p_{x}^{\prime 2}\right\rangle=\left(\frac{E_{0}}{c}\right)^{2} \epsilon_{x} \gamma_{x} \quad\left\langle p_{y}^{\prime 2}\right\rangle=\left(\frac{E_{0}}{c}\right)^{2} \epsilon_{y} \gamma_{y} \quad\left\langle p_{z}^{\prime 2}\right\rangle=\left(\frac{E_{0}}{c}\right)^{2}\left(\frac{\sigma_{\epsilon}}{\gamma}\right)^{2}
$$

where $\gamma_{x, y}$ are the beam ellipse parameters and the primes are used to denote quantities in the beam frame. The longitudinal momentum tends to be much smaller 
than the horizontal or vertical becallsc of the $1 / \gamma$ factor; for example, in the NLC damping ring, the ratio of the monenta is: $\left\langle p_{x}^{\prime 2}\right\rangle:\left\langle p_{y}^{\prime 2}\right\rangle:\left\langle p_{z}^{\prime 2}\right\rangle=7000: 35: 1$.

Actually, in $e^{+} / e^{-}$storage rings, the opening angle of the synchrotron radiation, see Section 2.2.3, imposes a lower bound on the transverse momenta such that $\epsilon_{x, y} \gamma_{x, y} \gtrsim \sigma_{\epsilon}^{2} / \gamma^{2}$. Thus, the transverse momenta are always greater than or comparable to the longitudinal $\left\langle p_{x, y}^{\prime}{ }^{2}\right\rangle \gtrsim\left\langle p_{c}^{\prime 2}\right\rangle$. Since the longitudinal direction is "cooler" than the transverse, one would expect the longitudinal momentum spread to increase at the expense of the transverse momenta. Unfortunately, this simple picture is complicated by the dispersion, which couples a change in the longitudinal momentum to the transverse planes. Thus, a scattering event that transfers transverse momentum to the longitudinal plane has both a cooling and a heating effect on the transverse phase space. The cooling is due to the direct exchange of momenta and the heating is due to the dispersion; it is important to realize that this heating is completely analogous to the heating clue to synchrotron radiation.

We can see this behavion in an estimate of the diffusion rates. Assuming that the two transver'se momenta are comparable and they are both much greater than the longitudinal, the diffusion rates are apjproximately ${ }^{(8)\}}$

$$
\begin{aligned}
& \frac{1}{\tau_{x, y \mathrm{IBS}}} \approx \frac{\sigma_{\epsilon}^{2}}{\epsilon_{x, y}}\left(\overline{\mathcal{H}_{x, y}}-\frac{1}{2 \gamma^{2}}\right) \frac{1}{\tau_{\epsilon} \mathrm{lBS}} \\
& \frac{1}{\tau_{\epsilon \mathrm{IBS}}} \approx \frac{c r_{0}^{2} N^{\prime}}{32 \gamma^{3} \sigma_{z} \sigma_{\epsilon}^{2} \epsilon_{x} \epsilon_{y}}\left(\frac{\epsilon_{x} \epsilon_{y}}{\overline{\beta_{x} \beta_{y}}}\right)^{1 / 4} \ln \left(\frac{\sigma_{y} \gamma^{2} \epsilon_{x}}{r_{0} \overline{\beta_{x}}}\right),
\end{aligned}
$$

where the diffusion rates are those of the rms values: $1 / \tau_{x, y, \varepsilon} \equiv 1 / \sigma_{x, y, \epsilon} d \sigma_{x, y, \epsilon} / d t$, $\mathcal{H}_{x, y}$ is a function of the dispersion, Eq. (A.5.9), and the values $\overline{\mathcal{H}}$ and $\bar{\beta}$ are averaged around the ring.

In an $e^{+} / e^{-}$storage ring, we can further simplify the expression for the transverse rates by realizing that the emititances are functions of the dispersion. In particular, the emittances can be cxpressed as

$$
\epsilon_{x, y} \sim \overline{\mathcal{H}_{x, y} \text { bends }} \sigma_{\ell}^{2} \frac{\mathcal{J}_{\epsilon}}{\mathcal{J}_{x, y}}
$$

where $\mathcal{J}_{x, y, \epsilon}$ are the damping partitions and $\overline{\mathcal{H}_{\text {bend }}}$ is averaged over the bourling 
magnets. Now, the transverse diflusion rales can be written:

$$
\frac{1}{\left.\tau_{x, y}\right] \mathrm{IBS}} \sim \frac{\overline{\mathcal{H}_{x, y}}}{\overline{\mathcal{H}_{x, y} \text { bends }}} \frac{\mathcal{J}_{\epsilon}}{\mathcal{J}_{x, y}} \frac{1}{\tau_{\epsilon} \text { IBS }}
$$

Typically, the damping partitions are in the range of one to two. Furthermore, in the vertical plane, the ratio $\overline{\mathcal{H}} / \overline{\mathcal{H}_{\text {bends }}}$ will be close to one since the vertical dispersion is due to error's. In contrast, the ratio is usually greater than one in the horizontal plane since the dispersion function is minimized in the bends.

Thus, Eq. (2.3.4) shows that the diffusion rates are comparable in all three planes. We can understand this simply by realizing that when $\left\langle p_{x, y}^{\prime}{ }^{2}\right\rangle \gg\left\langle p_{\mathrm{c}}^{\prime 2}\right\rangle$ the intrabeam scattering heating is analogous to the heating due to synchrotron radiation, except the scattering occurs everywhere around the ring and not just in the bending magnets. Since the low current equilibrium emittances are determined by the synchrotron radialion, the relative increase of the three emittances due to the intrabeam scattering must be comparable, $\Delta \epsilon_{x} / \epsilon_{x}$ SR $\sim \Delta \epsilon_{y} / \epsilon_{y} \mathrm{SR} \sim \Delta \epsilon_{z} / \epsilon_{z} \mathrm{SR}$; this must be true whether the vertical emittance is due to vertical dispersion or due to betatron coupling.

Finally, we should note hat the current theories of intrabeam scattering may over-estimate the real enittance growth. These theories estimate the rms emittance, but, with scattering, the beam distribution becomes non-gaussian and thus the rms emittance does not characterize the beam emittance well. This occurs because the infrequent hard scatterings can heavily bias the rms emittance and yet they only cause a halo of large amplitude particles; the hard scatterings do not affect the core emittance. A similar eflect occur's with beam-gas scattering and is described in greater detail there (Section 2.3.6).

\subsubsection{Small Vertical, Emittances}

Now, we will discuss modifications to this theory as the vertical emittance decreases. As the vertical cmittance gets smaller, the vertical diffusion rate must increase relative to the lorizontal. If the vertical emittance is comparable to the opening angle limit while the horizontal emittance is much larger, then there are two changes to the scattering rates of Ecl (2,3.2): first, since the vertical and longitudinal momenta are then comparable, the vertical plane is not cooled by the longitudinal. Second, since the erertical momentum distribution is much cooler than 


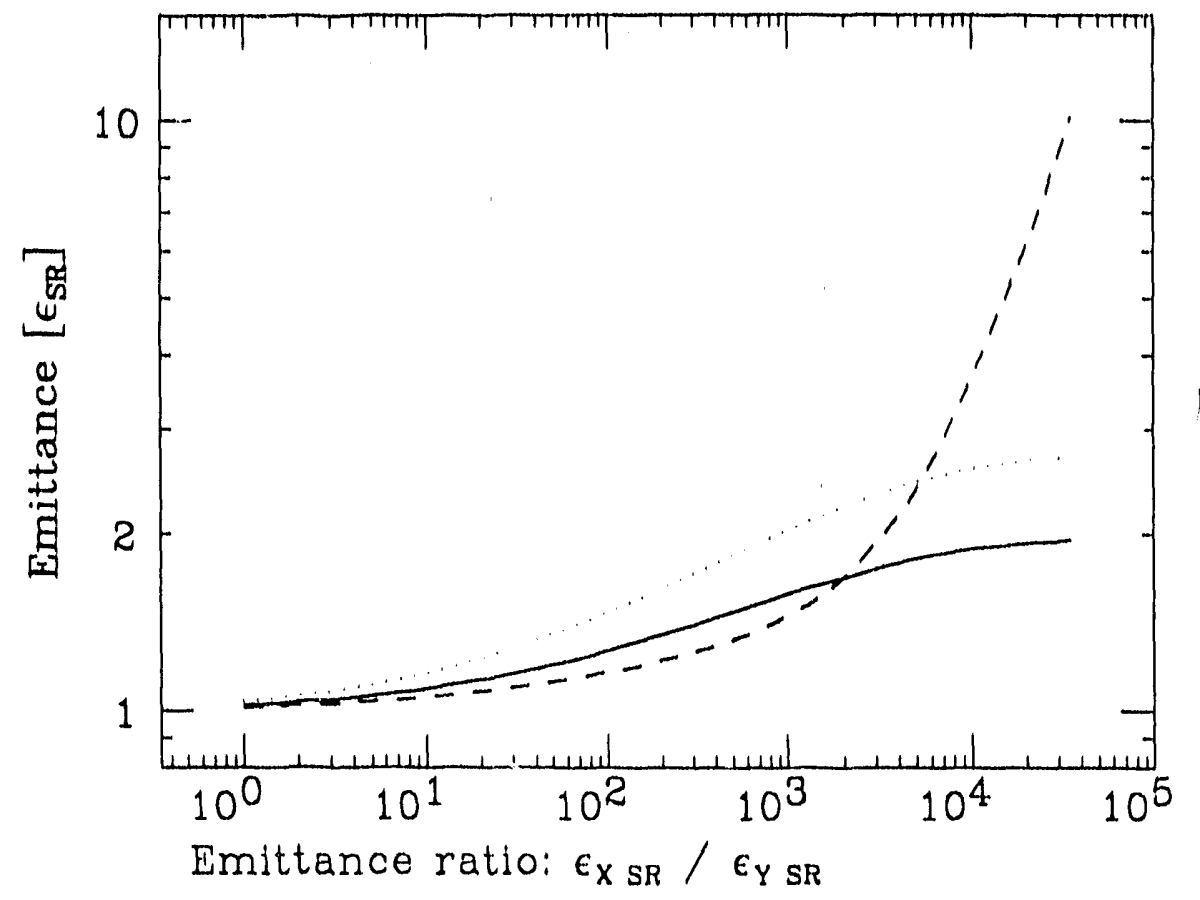

Fig. 13. Emittance increase due to IBS' v's, $\epsilon_{x} / \epsilon_{y}$. The solid, clashed, and dotted lines are the horizontal, vertical, and longitudinal emittances in units of the equilibrium emittance without IBSS.

the horizontal, there is additional heating of the vertical from the horizontal plane. These simple arguments suggest that the diffusion rates can be written:

$$
\begin{aligned}
& \frac{1}{\tau_{x \mathrm{IBS}}} \approx \frac{\sigma_{\epsilon}^{2} \overline{\mathcal{H}_{x}}}{\epsilon_{x}} \frac{1}{\tau_{\epsilon} \mathrm{IBS}} \\
& \frac{1}{\tau_{y} \mathrm{IBS}} \approx \frac{\sigma_{\epsilon}^{2}}{\epsilon_{y}}\left(\overline{\mathcal{H}_{y}}+\frac{1}{\gamma^{2}}\right) \frac{1}{\tau_{\epsilon} \mathrm{IBS}} \\
& \frac{1}{\tau_{\epsilon} \mathrm{IBS}} \approx \frac{c r_{0}^{2} N^{\prime}}{64 \gamma^{3} \sigma_{z} \sigma_{\epsilon}^{2} \epsilon_{x}=y}\left(\frac{\epsilon_{x} \epsilon_{y}}{\overline{\beta_{x} \beta_{y}}}\right)^{1 / 4} \ln \left(\frac{\sigma_{y} \gamma^{2} \epsilon_{x}}{r_{0} \overline{\beta_{x}}}\right),
\end{aligned}
$$

where we have used the fitct that the diffusion from the horizontal to the vertical is equal to the diffusion from the horizontal to the longitudinal when $\epsilon_{y} \gamma_{x, y} \sim \sigma_{\epsilon}^{2} / \gamma^{2}$.

These equations show that as $\epsilon_{y}$ clecreases, the vertical rate should increase relative to the horizontal. At the limit imposed by' the opening angle of the synchrotron radiation, the vertical scattering rate. and therefore, the increase of the vertical emittance, is roughly twice that of thr horizontal. 
This is illustrated in Fig, 13 where we have used the theory of Ref, 17 to calculate the equilibrlum emittances as a function of the aspect ratio $\epsilon_{x} \mathrm{SR} / \epsilon_{y} \mathrm{SR}$. The equilibrium emittance values are plotted in units of the equilibrim emittance due to synchrotron radiation $\epsilon \mathrm{SR}$. The emittance $\epsilon_{\mathrm{SR}}$ only includes contributions from the dispersion; the opening angle contribution has been neglected. Finally, we have used parameters of the NLC damping ring, in particular, $N=2 \times 10^{10}$ particles, $\gamma \epsilon_{x}=3 \times 10^{-6} \mathrm{mrad}$, and $\gamma=3522$.

One can see in Fig. 13 that when the aspect ratio is less than 1000, the relative emittance increase due to intrabeam scattering is comparable in all three planes; it increases slowly from a few percent to roughly $100 \%$. As the aspect ratio increases beyond 1000 , the vertical emittance starts to increase more rapiclly than the horizontal and longitudinal. At the opening angle limit, which occurs at an aspect ratio of 5000 , the vertical emittance has been increased by roughly $120 \%$ while the horizontal has increased only $80 \%$. At aspect ratios greater than 5000 , the vertical emittance rapidly increases while the horizontal and longitudinal emittances remain roughly constant; of course, this regime is not actually accessible since the opening angle limits the vertical emittance.

Thus, in $e^{+} / e^{-}$storage rings, intrabeam scattering is roughly as important in the vertical as in the horizontal. While intrabeam scattering becomes more important as the vertical emittance decreases, the scattering does not become significantly more detrimental in the vertical plane than in the horizontal. This occurs because, in $e^{+} / e^{-}$rings, the vertical emitlance cannot be clecreased beyond the limit due to the opening angle of the synchrotron radiation.

\subsubsection{DAMPING RATES}

Here, we will briefly discuss the effect of intrabeam scattering on the damping. Since intrabeam scattering causes a diffusion that counters the synchrotron radiation clamping, it is reasonalse to assume that it might slow the approach to equilibrium, in addition to increasing the equilibrium value. We start with the differential equation for the enittance which can be written

$$
\frac{d \epsilon(t)}{d t}=-\frac{2 \epsilon(t)}{\tau_{\mathrm{SR}}}+\frac{2 \epsilon(t)}{\tau_{\mathrm{IBS}}\left(\epsilon_{x}, \epsilon_{y}, \epsilon_{z}\right)}+\frac{2 \epsilon_{\mathrm{SR}}}{\tau_{\mathrm{SR}}} .
$$

Here, the first term is clue to the symchrotron radiation damping while second and third terms are due to the intrabean scattering diffusion and the synchrotron radia- 


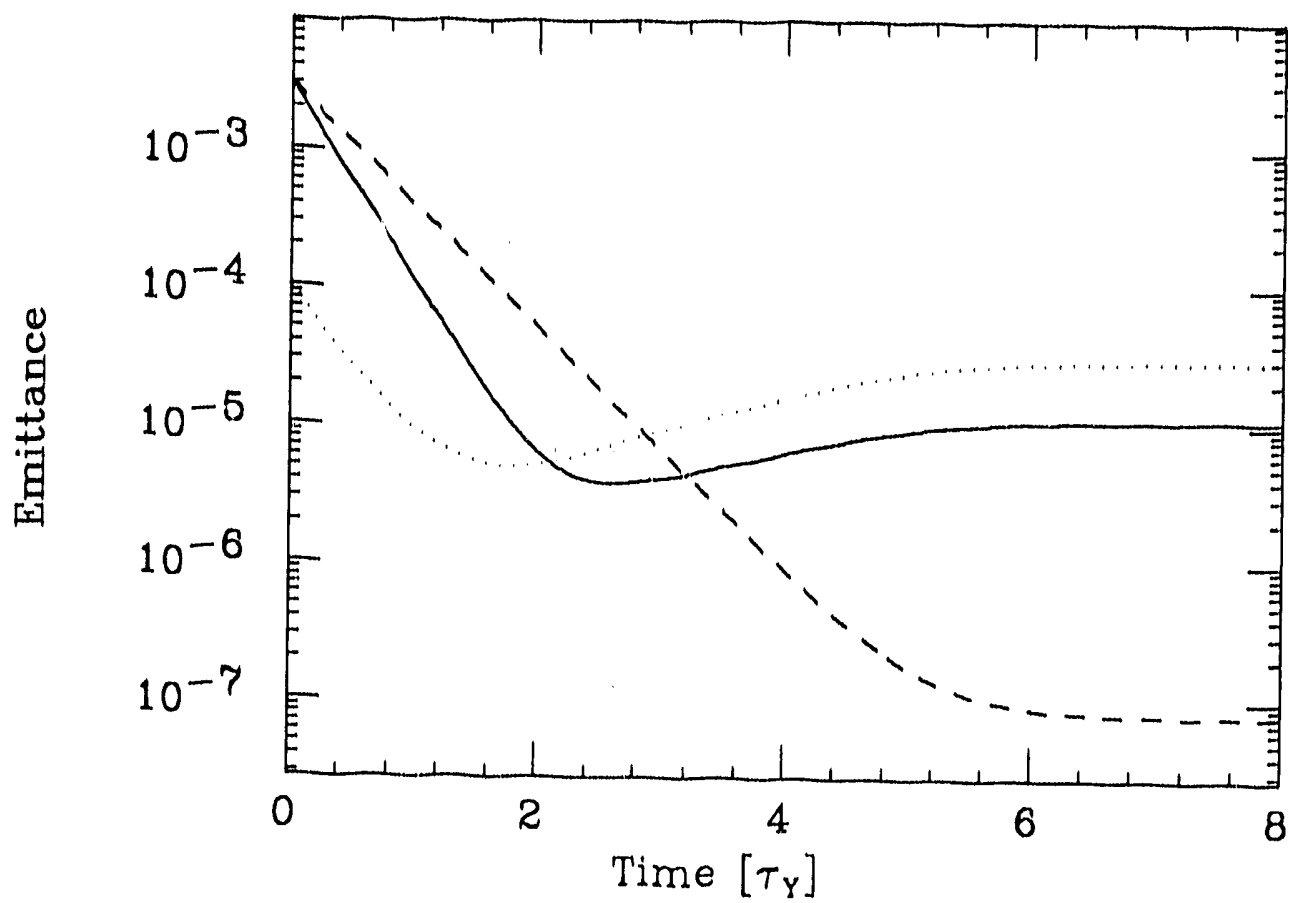

Fig. 14. Emittance vs. storage time in the NLC damping ring with $4 \times 10^{12}$ $e^{+} / e^{-}$. The solid, dashed, and dotted lines are $\gamma \epsilon_{x}, \gamma \epsilon_{y}$, and $\epsilon_{z}$, respectively.

tion (quantum) diffusion; in the last term, $\epsilon \mathrm{SR}$ is a constant equal to the equilibrium emittance due to the synchrotron racliation.

The intrabeam scattering diffusion rate is inversely proportional to the emittances. If we assume that all three emittances approach equilibrium at the same time, then the intrabeam scattering rate increases as $1 / \epsilon^{3}$. Thus, the emittance approaches the equilibrium value faster than it does when intrabeam scattering is negligible; of course, the equilibrium emittance is larger with intrabeam scattering than without intrabeam scattering.

If the horizontal and longitudinal emittance reach equilibrium before the vertical, as is the case in the NLC damping ring, then the horizontal and vertical emittance will be increased as the vertical clamps to its equilibrium. This occurs because the intrabeam scattering becomes more important as the vertical continues to damp. Thus, using the argument above, one would expect the damping rate to be decreased as the vertical emittance approaches equilibrium.

Actually, in calculations using the theory of Ref. 17, we find that the vertical damps faster with intrabeam scattering thian without. An example is illustrated in Fig. 14 where we have plotted $\gamma \epsilon_{x}, \gamma \epsilon_{y}$, and $\sigma_{z} \sigma_{\epsilon}$ as a function of time in the NLC 
damping ring. To exaggerate the effect of the intrabeam scattering, we have used a current of $4 \times 10^{12} e^{+} / e^{-}$per bunch and thus the horizontal and longitudinal emittance increase noticeably as the vertical emittance damps.

In Fig. 14, the vertical emittance damps faster with intrabeam scattering than without because the intrabeam scattering provides additional damping in the vertical plane when the vertical emittance is larger than the horizontal and longitudinal emittances. For example, after five damping times, the vertical emittance is $96 \%$ of the value it would be without the intrabeam scattering. Of course, this is a small effect and at lower currents, intrabeam scattering has no noticeable effect on the damping rates.

\subsubsection{SCALing With ENERGy}

Finally, we will examine the dependence of the intrabeam scattering on the ring energy. The diffusion rates are given by Eqs. (2.3.2) or (2.3.5). Since these expressions depend inversely upon $\gamma^{3}$, it is standard to state that intrabeam scattering becomes insignificant as the beam energy increases. But, in a damping ring, we are interested in the normalized emittances: $\gamma \epsilon_{x, y}$ and $\gamma \sigma_{z} \sigma_{\epsilon}$. Expressing Eq. (2.3.2) in terms of these values, we find

$$
\begin{aligned}
& \frac{1}{\tau_{\epsilon} \text { IBS }} \propto \frac{N}{\sqrt{\gamma} \sigma_{\epsilon}\left(\gamma \sigma_{z} \sigma_{\epsilon}\right)\left(\gamma \epsilon_{x}\right)^{3 / 2}} \ln () \\
& \frac{1}{\tau_{x \mathrm{IBS}}} \propto \frac{N \mathcal{H}_{x}}{\sqrt{\gamma} \sigma_{z}\left(\gamma \epsilon_{x}\right)^{5 / 2}} \ln () .
\end{aligned}
$$

In terms of these normalized quantities, the scattering rates are only weakly dependent on the energy.

Of course, we are actually interested in the effect of intrabeam scattering on the emittances. This depends on the ratios of the intrabeam diffusion rates to the synchrotron radiation clamping rates. If we naively assume a scaling such that the important parameters of the ring, namely the normalized emittances and the effective damping rates, remain constant, see Eqs. (A.6.6) and (A.6.7), we find that $\mathcal{H}_{x}$ also remains constant while the bending radius and the damping times increase with $\gamma^{3}$. This implies that the effect of the intrabeam scattering increases as $\gamma^{3}$ and thus lower energies are better! 
Obviously, one can be far more clever in the scaling, but it is important to realize that, when designing a ring with specified nowmalized emittances, simply increasing the beam energy does not imply that the intrabeam scattering can be neglected. Although, initially this result may seem counter-intuitive, one has to remember that the scattering is inversely dependent on the normalized six-dimensional phase space volume of the beam. If this is held constant, then the effect of the scattering will only depend upon the synchrotron radiation damping rates.

\subsubsection{Ion Trapping}

The particle beam will jonize the residual gas in the vacuum chamber. In the case of an electron beam, these ions can be trapped in the negative potential well created by the circulating electron beam. The trapped jons will then cause large tune shifts in the stored beam, possibly leading to beam loss. In addition, the ion potential is highly nonlinear and thus it can drive high order resonances. Finally, the ions can also drive collective instabilities of the electron beam. In all cases, the net effect is to limit the beam current and increase the beam emittances.

We can estimate the stability of the ions by assuming that the circulating electron bunches act like thin-lense focusing quadrupoles. Then, the problem becomes that of analyzing betatron stability where the focusing is due to the space charge field of the electrons. To obtain an initial estimate, we assume equally spaced bunches with equal charge and dimensions. In this case, one finds that, for stability. the ions must have atomic weights greater illan ${ }^{[c]}$

$$
A=\frac{N r_{p}}{n_{b}} \frac{C}{4 \sigma_{y}\left(\sigma_{x}+\sigma_{y}\right)}
$$

where $n_{b}$ is the number of bunches, $r_{p}$ is the proton classical radius, and $C$ is the ring circumference. Notice that the minimum stable ion mass is inversely proportional to the vertical beam size; this is probably the only detrimental effect that actually eases as the beam size decreases.

Equation (2.3.8) shows that the minimum atomic weight is inversely proportional to the vertical beam size. Thus, we can estimate the worst case for the NLC damping ring by calculating the minimum atomic weight of ions that are trapped when a batch of electrons is injected into the ring. A batch contains ten closely spaced bunches of $2 \times 10^{10}$ electrons; in this simple estimate we will treat this as a 


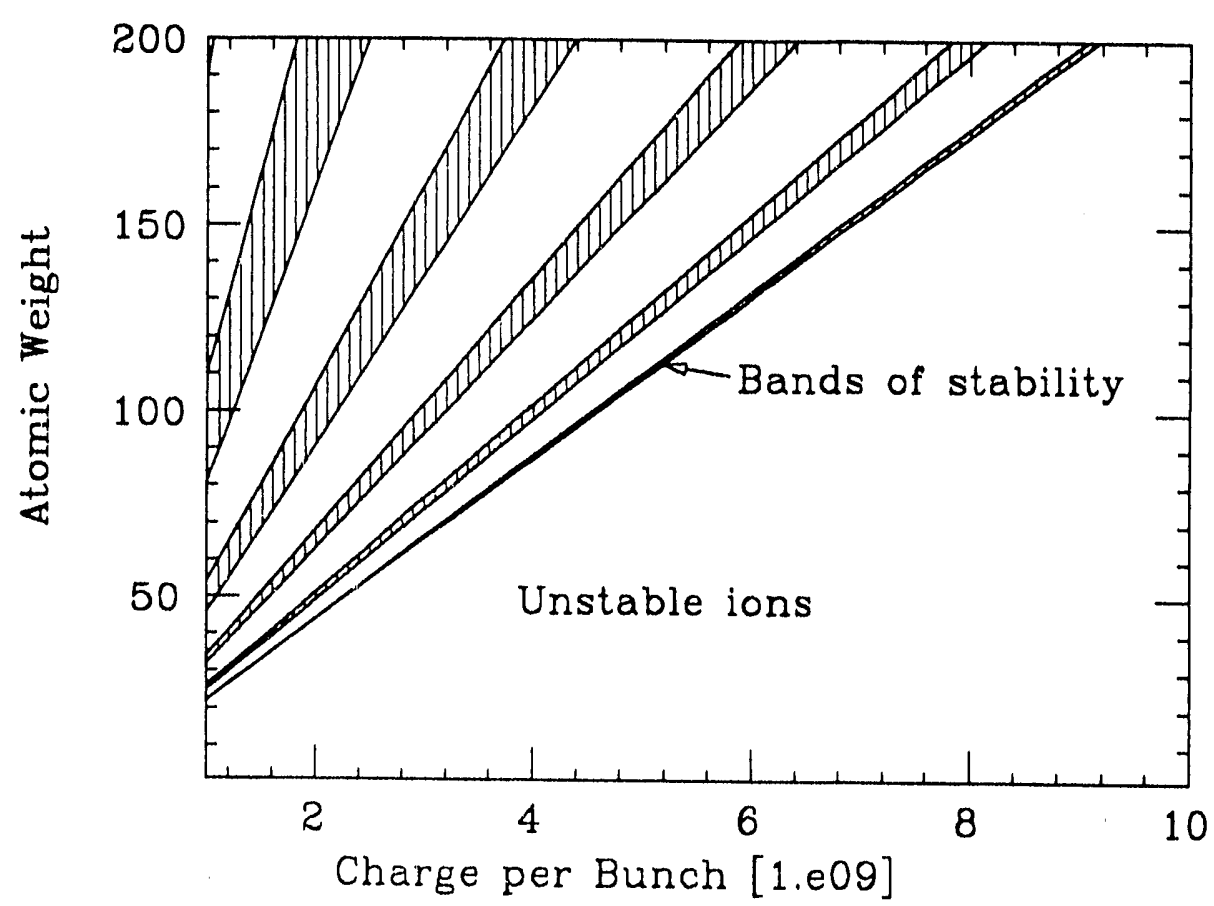

Fig. 15. Bands of stable ion masses in the NLC damping ring as a function of the charge per bunch assuming ten batches of ten bunches.

single bunch of $2 \times 10^{11}$ electrons. With these values, $\mathrm{Eq}$. (2.3.8) specifies a minimum atomic weight of ten. In a typical vacuum system, one finds ions with atomic masses ranging from 1 to 44 . Obviously, this indicates that ions might be a problem before the beam damps.

Of course, in operation the NLC damping ring will contain ten batches of bunches with beam sizes ranging from that of a fully damped beam to that of an injected beam. Equation (2.3.8) camnot be used in this case, but one can calculate the linear stability in the same fashion. Although the theory is simple, the computation is complex and one cannot express the result in a simple formula. Despite this, the scaling of the result is the same as given by Eq. (2.3.8). Thus, lowering the bunch population decreases the threshold for trapping while decreasing the number of batches and decreasing beam sizes increases the threshold for trapping.

Calculating with ten batches of ten bunches of $2 \times 10^{10}$ electrons, we find stable bands of jons with masses greater than 440; obviously, this is not a problem. But, if the bunch population is decreased, as it undoubtedly will when commissioning the ring, the minimum stable ion mass also decreases. In Fig. 15, we have plotted the regions of stability as a function of the current per bunch for the NLC damping 
ring, assuming ten batches of ten bunches. When the current is decreased to $1 \times 10^{9}$ electrons per bunch, one finds stable regions where the ions typically found in storage rings could be trapped. Fortunately, these bands of stability are narrow. Since the bunches in the ring are continuously damping, the regions of stability change. Thus, this linear theory indicates that it is unlikely that significant accumulation could occur, even at very low currents.

Of course, we should note that we have assumed a linear restoring force while the actual space charge force is highly nonlinear, dropping to zero at large distances. Although, the nonlinearity could generate large amplitude stable islands, even when the linear motion is unstable, nonlinear simulations are in extremely good agreement with the linear theory, ${ }^{[18,61,83]}$ indicating that the linear theory is sufficient.

Finally, wigglers in the ring can act like magnetic bottles, thereby increasing the trapping. These effects can be analyzed with detailed computer programs. However, in the case of the NLC damping ring, jons are unlikely to be a problem since, at the design current, the ions are very far from linear stability.

\subsubsection{Space Charge}

In this section, we will discuss incolyerent effects due to the electromagnetic field of the beam. We will treat the beam as if it were in free space, neglecting the modification of the fields by the vacuum chamber; these will be treated in Sections 2.3.4 and 2.3.5. The primary effect of the space charge field is to cause an amplitude dependent tune shift. We will calculate this and estimate its effects. In addition, we will estimate the betalron coupling introduced by the space charge field; this could be relevant for flat beams.

\subsubsection{Space Charge Field}

The space charge field will modify the forces felt by the particles. Like the beambeam force, the force particles experience when two colliding beams pass through each other, the space charge force is highly nonlinear. Unlike the beam-beam force which is discrete, the space charge force is continuous and thus it will not tend to drive high-order resonances.

The space charge field for a gaussian bunch, whose transverse dimensions are small compared to the longitudinal, is given in Ref. 12. This is illustrated in Fig. 16, where we have plotted the vertical electric field versus the vertical position for a 


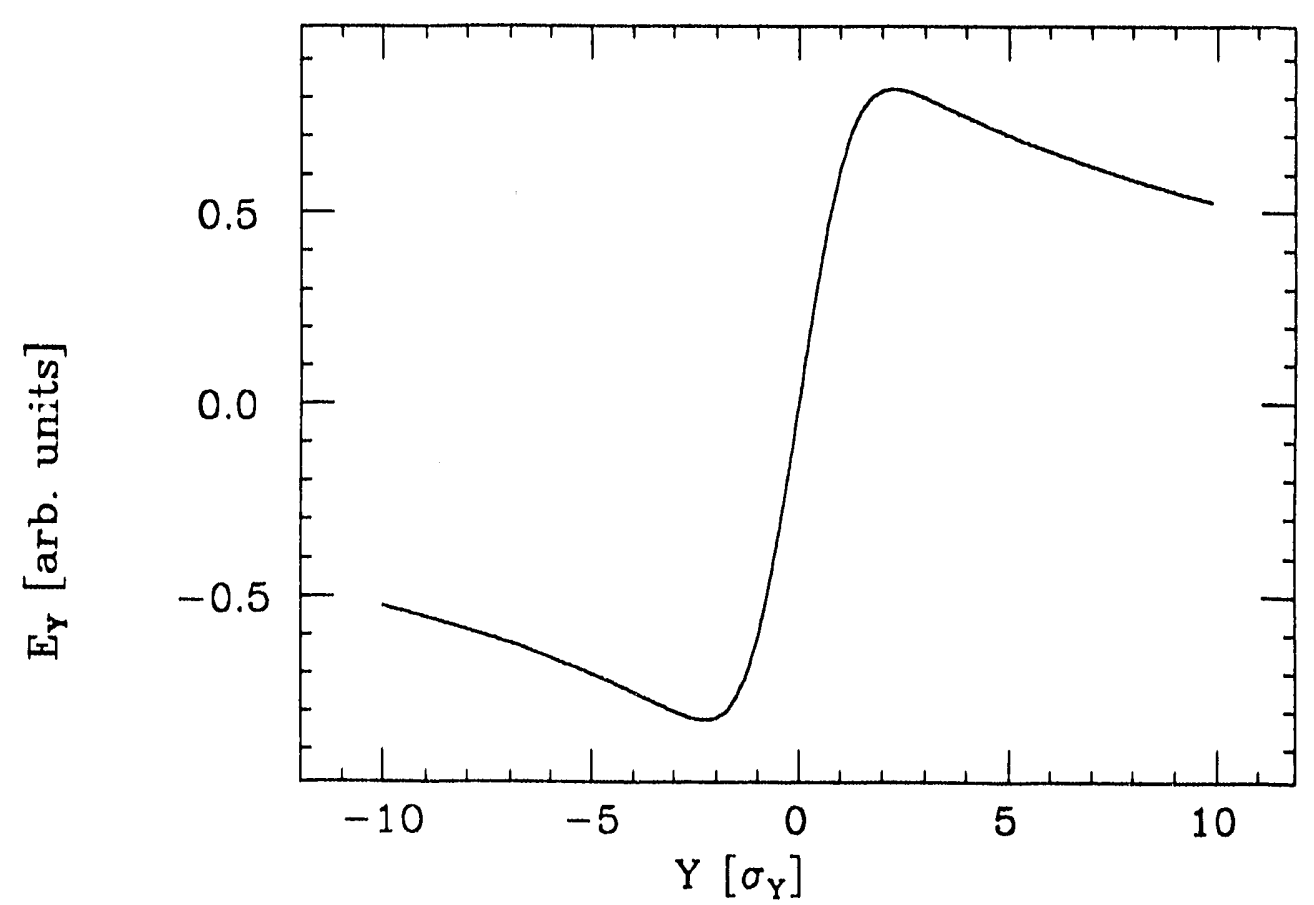

Fig. 16. Space charge field for $\sigma_{x} / \sigma_{y}=10: 1$.

bunch with an aspect ratio $\sigma_{J^{\prime}} / \sigma_{y}$ of $10: 1$. Obviously, the space charge field is highly nonlinear. 'The field clianges rapidly' as one passes through the center of the bunch and then decays slowly at amplitudes greater than roughly $\pm 2 \sigma_{y}$.

We can expand the Lorentz force in a power series in $x$ and $y$. In a gaussian bunch, the vertical force is

$$
F_{y}(z)=\gamma m c^{2} e^{-z^{2} / 2 \sigma_{z}^{2}} \sum_{i, j} F_{y i j} x^{i} y^{j},
$$

where $\gamma m c^{2}$ is the electron energg: $z$ is the longitudinal position within the bunch, and the first couple of cocficicnts $F_{y}$ ij are

$$
\begin{aligned}
& F_{y 01}=\frac{2 N r_{0}}{\sqrt{2 \pi} \sigma_{z} \gamma^{3}} \frac{1}{\sigma_{y}\left(\sigma_{x}+\sigma_{y}\right)} \\
& F_{y 21}=-\frac{N r_{0}}{\sqrt{2 \pi} \sigma_{z} \gamma^{3}} \frac{1}{\sigma_{x} \sigma_{y}\left(\sigma_{x}+\sigma_{y}\right)^{2}} \\
& F_{y 03}=-\frac{N r_{0}}{3 \sqrt{2 \pi} \sigma_{z} \gamma^{3}} \frac{\left(\sigma_{x}+2 \sigma_{y}\right)}{\sigma_{y}^{3}\left(\sigma_{x}+\sigma_{y}\right)^{2}} ;
\end{aligned}
$$

with similar expressions for the horizontal plane. 
These coefficient $F_{y i j}$ are analogous to the magnetic field coefficients $K_{n}$ that are used to specify the magnetic multipole strengths. The first term $F_{y 01}$ causes a linear tune shift similar to a quadrupole magnet while the second and third terms cause an amplitude dependent betatron coupling and an amplitude dependent tune shift having effects similar to those of an octupole magnet.

\subsubsection{INCOHERENT TUNE SHIFT}

We can estimate the magnitude of the space charge force with the linear tune shift; this is usually referred to as the direct incoherent Liaslett tune shift. ${ }^{[6]]}$ Assuming gaussian bunches, the maximum tune shift occurs at the center of the bunch; it is given by ${ }^{[34]}$

$$
\Delta \nu_{y}=-\frac{1}{4 \pi} \int_{0}^{c_{1}} d s \beta_{y} F_{y 01}
$$

with a similar expression for the horizontal. This yields tunes shifts of

$$
\Delta \nu_{x, y}=-\frac{N r_{0} \overline{\beta_{x, y}} R}{\sqrt{2 \pi} \gamma^{3} \sigma_{z}} \frac{1}{\sigma_{x, y}\left(\sigma_{x}+\sigma_{y}\right)},
$$

where $R$ is the average radius of the ring: $R=C / 2 \pi$. Notice that it is inversely proportional to the beam dimensions and thus $\Delta \nu_{y}$ becomes important as the vertical beam size shrinks. In addition, notice that the tune shift is amplitude dependent. It strongly depends upon the amplitude of the longitudinal and horizontal motion and, in the flat beam case, is more weakly dependent on the amplitude of the vertical motion. The transverse amplitude dependence stabilizes the beam to high order resonances while the longitudinal amplitude dependence causes the tune shift to be modulated by the synchrotron motion: this is discussed in Section 2.2.6.

In the NLC damping ring, we find a vertical tune shift of $\Delta \nu_{y}=-0.015$ while the horizontal tune shift is an order of magnitude smaller. Although this is much larger than is typical in electron storage rings, it should not present a problem. Proton rings frequently run with space charge tune shifts that are larger by more than an order of magnitude. The primary effect of this tune shift is to move particles onto resonances, see Section 2.2.6. Thus, provided that the tunes are far from the low-order resonances, the space charge tume shift should be a negligible effect. 
Finally, we should note that the space charge does not affect rigid dipole oscillations of the beam ${ }^{(102)}$ since the beam drags it's self-field with it. Thus, the space charge induced tune spread will not cause a coherent betatron oscillation to decohere. This is important when discussing filamentation and the effect of coherent jitter.

\subsubsection{Betatron Coupling}

The space charge field of any non-uniform bunch has skew components that could drive betatron coupling resonances. Obviously, this could be important when siriving for very dense asymmetric beams. This coupling was first calculated in Ref. 75 with reference to low energy proton beams. There, the smooth approximation was used to calculate the single particle behavior including all components of the space charge potential through fourth order. Unfortunately, this analysis is rather complex and does not lend itself to a simple estimate of when the effect becomes important for flat beams.

Here, we will estimate the magnitude of the space charge coupling in the flat beam limit where the vertical motion does not affect the horizontal. We will use the smooth approximation and consider only the effect of the lowest order coupling resonance; in a gaussian beam this is the $2 \nu_{x} \pm 2 \nu_{y}$ resonance. To estimate the emittance dilution, we assume that the ring is far from the coupling resonances and we use first order perturbation theory, neglecting all de-tuning effects; obviously, such an analysis is only valid when the space charge forces are weak. The other limit, where one is close to the coupling resonance, is analyzed in Section 3.9.1, with reference to transport in linacs.

In a gaussian beam, the vertical space charge force is given by Eq. (2.3.9). In the smooth approximation, this yields a differential equation for $y$ as a function of $\theta$, the azimuthal position around the ring:

$$
\frac{d^{2} y}{d 0^{2}}+\nu_{y}^{2} y=\sum_{i, j} R^{2} F_{y i j} x^{i} y^{j}
$$

where $\nu_{y}$ is the vertical tune and the first couple of coefficients $F_{y i j}$ are given in Eq. (2.3.10); the lowest order coupling resonance is driven by the $F_{y 21}$ term.

Now, we use first order perturbation theory to solve for the increased beam size due to the coupling. The vertical position is $y=y^{(0)}+y^{(1)}$ where $y^{(0)}$ and $x^{(0)}$ 
describe the unperturbed motion. These are

$$
x^{(0)}=\sqrt{2 J_{x} \beta_{x}} \cos \left(\nu_{x} \theta+\phi_{x}\right) \quad \text { and } \quad y^{(0)}=\sqrt{2 J_{y} \beta_{y}} \cos \left(\nu_{y} \theta+\phi_{y}\right)
$$

where $J_{x, y}$ are the single particle actions, $E_{q} .(A .2 .21), \phi_{x, y}$ are the conjugate phases, and, in the smooth approximation, $\beta_{x, y}=R / \nu_{x, y}$. The equation for the lowest order coupling contribution is

$$
\frac{d^{2} y^{(1)}}{d \theta^{2}}+\nu_{y}^{2} y^{(1)}=R^{2} F_{y 21} x^{(0) 2} y^{(0)}
$$

Provided that $\nu_{x}, \nu_{y},\left|\nu_{x} \pm \nu_{y}\right| \gg \Delta \nu$, we can neglect the tune shifts and the inhomogeneous solution is

$$
y^{(1)}=-\frac{R^{2} F_{y 12} J_{x} \beta_{x} \sqrt{2 J_{y} \beta_{y}}}{8 \nu_{x}\left(\nu_{x} \pm \nu_{y}\right)} \cos \left(\left(\nu_{y} \pm 2 \nu_{x}\right) \theta+\phi_{y} \pm 2 \phi_{x}\right)
$$

Notice that the solution depends upon the full tunes, not just the fractional portions. This occurs because the force was assumed to be constant around the ring and thus only the zeroth harmonic is driven.

At this point, we can find the emittance increase by averaging $y^{2} / \beta_{y}$ over the phases $\phi_{x, y}$. Assuming a gaussian beam distribution, this yields

$$
\frac{\Delta \epsilon_{y}}{\epsilon_{y}} \approx 2\left(\frac{\Delta \nu_{y}}{16} \frac{\nu_{y}}{\nu_{x}\left(\nu_{x} \pm \nu_{y}\right)}\right)^{2}
$$

where we have used the linear tune shift, Eq. (2.3.12), to simplify the expression. This clearly shows that this effect is only' significant when the tune difference $\nu_{x}-\nu_{y}$ is comparable to the linear space charge tune shift. In the NLC damping ring, the tune difference is roughly thirteen while the Laslett tune shift is 0.02 ; thus the emittance increase is negligible.

Finally, we can generalize this result to include the variation of the space charge force around the ring. We express the force in a Fourier series

$$
F_{y 21}(0)=\sum_{k=-\infty}^{\infty} f_{k} e^{i k \theta}
$$

where the magnitudes of the coefficients $\left|f_{k}\right|$ must be less than or equal to the d.c. term $\left|f_{0}\right|$, since $F_{y 21}$ does not change sign. This allows us to express the enillance 
increase due to the $k$ th harmonic as

$$
\frac{\Delta \epsilon_{y}}{\epsilon_{y}} \lesssim 2\left(\frac{\Delta \nu_{y} \nu_{y}}{4\left(k \pm 2 \nu_{x}\right)\left(k+2 \nu_{y} \pm 2 \nu_{x}\right)}\right)^{2}
$$

Again, this will be a negligible contribution, although it does suggest against tunes such that $2 \nu_{x} \pm 2 \nu_{y} \approx n p$, where $n$ is an integer and $p$ is the periodicity of the ring.

\subsubsection{Wakefields: Static Effects}

Wakefields and image forces arise from the electromagnetic interaction between the beam and the external environment. They occur when the direct space charge field is modified to meet the boundary conditions imposed by the vacuum chamber. In this section, we will only treat the static effects of the wakefields where the wakefield distorts the equilibrium beam distribution; we discuss the dynamical effects, which can lead to coherent instabilities, in the next section. In both sections, we will only treat a few issues which are pertinent to the vertical emittance. The beam-environment interaction is a very rich topic and we certainly make no attempt to cover it in detail.

As was mentioned in Section 2.2.6, the longitudinal wakefield in the presence of dispersion and the transverse dipole wakefield due to a non-zero closed orbit can cause synchro-betatron coupling in a single bunch; this occurs because the wakefield deflection is a function of the longitudinal position $z$ within the bunch. In addition, the transverse quadrupole waliefield can contain skew quadrupole components that induce transverse betatron coupling. Finally, there are higher order wakefield contributions. However, provided that the orluit offsets are small compared to the vacuum chamber radius, these will be much smaller.

We will estimate these effects after describing the origin of the wakefields in a bit more detail. We will only consider the effects in a single bunch, assumis g that the wake is damped between bunches. Of course, there are multi-bunch analogs to each of these effects. For example, the longitudinal wakefield, in regions of dispersion, or the dipole transverse wakefield can increase the effective emittance of a train of bunches, since each bunch will receive a different deflection and thus the closed orbit of each bunch will differ. 


\subsubsection{WAKEFIELDS}

In free space, the electromagnetic field of an ultra-relativistic particle is Lorentz contracted into a thin disk purpendicular to the particle motion. Similarly, if the particle is propagating inside a perfectly conducting vacuum chamber, the electromagnetic field is still Lorentz contracted into a thin disk, although now, the transverse field is modified to meet the boundary conditions at the chamber wall. These modifications are due to image currents that flow on the inside surface of the vacuum chamber.

This changes if the vacuum chamber has finite conductivity or if the chamber changes cross section. In this case, the electromagnetic field acquires a longitudinal component; it trails the generating charge. This is called a wakefield and is important because it provides a mechanism for a particle to affect both other trailing particles and itself on subsequent revolutio'ss.

The wakefield is typically described in either the time domain with the wake function $W$ or in the frequency domain with the impedance $Z$; the impedance is simply the Fourier transform of the walie function. The wake function is proportional to the Lorentz force experienced by a test charge a distance $\Delta z$ from the point charge generating the field. Obviously, causality requires that the wakefield of an ultra-relativistic particle must be zero when $\Delta z>0$.

We will consider both the wakefield clue to the finite conductivity of the vacuum chamber and the wakefield due to RF cavities. For values of $\Delta z=z^{\prime}-z$ in the range:

$$
\left(\frac{b^{2}}{\sigma Z_{0}}\right)^{1 / 3} \ll \Delta z \ll \sigma b^{2} Z_{0}
$$

the wake force due to the resistive wall of a cylindrical vacuum chamber can be expressed in a simple analytic form ${ }^{[2]}$

$$
\begin{aligned}
& \frac{F_{\| 0}(\Delta z)}{m c^{2}}=\frac{N r_{0}}{\pi b} \sqrt{\frac{\pi}{\sigma Z_{0}}} \frac{\hat{z}}{\Delta z^{3 / 2}} \\
& \frac{F_{\perp 1}(\Delta z)}{m c^{2}}=\frac{4 N^{\prime} r_{0} y^{\prime}}{\pi b^{3}} \sqrt{\frac{\pi}{\sigma Z_{0}}} \frac{\hat{y}}{\sqrt{\Delta z}} \\
& \frac{F_{\perp 2}(\Delta z)}{m c^{2}}=\frac{8 \Lambda^{\prime} r_{0} x^{\prime} y^{\prime}}{\pi b^{5}} \sqrt{\frac{\pi}{\sigma Z_{0}}} \frac{\left(y^{\prime} \hat{x}+x^{\prime} \hat{y}\right)}{\sqrt{\Delta z}} .
\end{aligned}
$$

We have listed the monopole longitudinal, the vertical dipole, and the skew quadru- 
pole wakefield forces scaled by $m c^{2}$. Here, $b$ is the vacuum chamber radius, $\sigma$ is the conductivity, $Z_{0}$ is the impedance of free-space, and the primed coordinates denote the position of the generating charge while the un-primed coordinates denote the position of the test charge.

Unfortunately, one cannot find a similar simple expression for the wakefields due to a cavity. Here, one typically uses a computer program to find the modes of oscillation for the cavity and then the wake function can be written as a sum over these modes. An example is illustrated in Fig. 51 (Appendix D), where we have plotted the longitudinal and the transverse dipole wake functions for the $11.4 \mathrm{GHz}$ NLC accelerator structure. ${ }^{[10]}$

\subsubsection{Longitudinal and Transverse Dipole Wakefields}

As discussed, the transverse dipole wakefield and the longitudinal wakefield in regions of dispersion will cause synchro-betatron coupling. When the synchrotron tune is very small, this coupling simply causes the beam to tilt in the $y-z$ and $y^{\prime}-z$ planes; the particles have different closed orbit depending upon their longitudinal position $z$ in the bunch. Here, we will estimate the effect of the transverse dipole wakefield, due to both the vacum chamber resistance and the RF cavities, using the results of Section 2.2.6.1; these two sources should provide a good (factor of two) estimate of the transverse wakefield. Effects of the longitudinal wakefield can be estimated in a similar manner.

Resistive Wall

To use the results of Section 2.2.6.1, we need to express the deflection due to the wakefields as a function of the longitudinal position in the bunch. This is found by integrating the wakeficld over the bunch distribution:

$$
\frac{d^{\prime} y(z)}{d s^{2}}=\int_{z}^{\infty} d z^{\prime} \rho\left(z^{\prime}\right) \frac{F_{\perp 1}\left(z^{\prime}-z\right)}{\gamma m c^{2}},
$$

where $\rho$ is the particle distribution in $z$. To perform this integration for the resistive wall wakefield, we need to know the wakefield from $z=0$ to $z=\infty$. Fortunately, simply integrating the approxination in Eq. (2.3.21) will yield negligible error ${ }^{[\theta]}$ provided that $\sigma_{z} \gg\left(b^{2} / \sigma Z_{0}\right)^{1 / 3}$. In a copper vacuum chamber with a $1 \mathrm{~cm}$ radius, this requires that $\sigma_{z} \gg 17 \mu m$ : this is certainly satisfied in the NLC damping ring where the rms bunch length is rougluy $5 \mathrm{~mm}$. 
Thus, assuming a gaussian longitudinal distribution, we find a deflecting force:

$$
\frac{d^{2} y(z)}{d s^{2}}=\frac{N r_{0} y^{\prime}}{\gamma \pi b^{3} \sigma_{z}} \sqrt{\frac{8}{\sigma Z_{0}}} \int_{z}^{\infty} \frac{e^{-z^{\prime 2} / 2 \sigma_{z}^{2}}}{\sqrt{z^{\prime}-z}} d z^{\prime}
$$

Now, to obtain the coefficients $g_{n}$ needed in Eq. (2.2.84), we can expand in a Taylor series about $z=0$ :

$$
\begin{aligned}
\frac{d^{2} y(z)}{d s^{2}} & =\sum_{n} g_{n} z^{n} \\
& =\frac{N r_{0} y_{c}}{\gamma \pi b^{3} \sigma_{z}} \sqrt{\frac{8}{\sigma Z_{0}}} \sum_{n} \frac{z^{n}}{n !}\left(\frac{d^{(n)}}{d z^{(n)}} \int_{0}^{\infty} \frac{d u}{\sqrt{u}} e^{-(u+z)^{2} / 2 \sigma_{z}^{2}}\right)_{z=0}
\end{aligned}
$$

where we have replaced $y^{\prime}$ with $y_{c}$, the closed orbit offset. This yields the coefficients:

$$
\begin{aligned}
& g_{1}=-\frac{\Gamma\left(\frac{3}{4}\right)}{\left(2 \sigma_{z}^{2}\right)^{1 / 4}} \frac{N r_{0} y_{c}}{\gamma \pi b^{3} \sigma_{z}} \sqrt{\frac{8}{\sigma Z_{0}}} \\
& g_{2}=-\frac{\pi}{\left(8 \sigma_{z}^{2}\right)^{3 / 4} \Gamma\left(\frac{3}{4}\right)} \frac{N r_{0} y_{c}}{\gamma \pi b^{3} \sigma_{z}} \sqrt{\frac{8}{\sigma Z_{0}}} \\
& g_{3}=\frac{\Gamma\left(\frac{3}{4}\right)}{2\left(2 \sigma_{z}^{2}\right)^{5 / 4}} \frac{N r_{0} y_{c}}{\gamma \pi b^{3} \sigma_{z}} \sqrt{\frac{8}{\sigma Z_{0}}},
\end{aligned}
$$

where $\Gamma$ is the gamma function and $\Gamma\left(\frac{3}{4}\right)=1.22 \cdots$.

Now, to calculate the coupling coefficient $S_{ \pm}\left(\mathrm{Eq} \cdot\left(2.2 .86^{\circ}\right)\right)$, we nifed the closed orbit. We shall approximate this assuming that the orbit is corrected to a random offset at each of the focusing quadrupoles. In this case, the value of $\left|S_{ \pm}\right|^{2}$ is approximately:

$$
\left|S_{ \pm}^{(n)}\right|^{2} \approx \frac{\beta_{y}}{\Lambda_{\mathrm{QF}}} g_{n}^{2}\left(y_{\mathrm{crms}}\right) C^{2}
$$

where $C$ is the ring circumference, $y_{c \mathrm{~m}}$ is the rms of the closed orbit, and $N_{\mathrm{QF}}$ is the number of focusing quadrupoles in the ring.

Finally, we can estimate the projected emittance. We will only estimate the lowest order coefficient since the higher-order contributions tend to be small unless they are on resonance. In the NLC , these resonances are very high-order ( $\sim 20$ th) 
and thus they will be very narrow. The NLC design calls for $2 \times 10^{10}$ particles per bunch with a $5 \mathrm{~mm}$ rms bunch length at $1.8 \mathrm{GeV}$. Assuming a copper vacuum chamber $\left(\sigma=5.8 \times 10^{7} \mathrm{mhos} / \mathrm{m}\right)$ with a $1 \mathrm{~cm}$ radius and a $100 \mu \mathrm{m}$ rms orbit with 75 QFs, we find:

$$
\left|S_{ \pm}^{(1)}\right|^{2} \approx 5 \times 10^{-13} \mathrm{~m}^{-1}
$$

Now, using Eq. (2.2.84), we find an increase in the projected vertical emittance of:

$$
\frac{\left\langle y^{2}\right\rangle}{\beta_{y}} \sim 5 \times 10^{-18} \mathrm{~m}-\mathrm{rad} .
$$

This is six orders of magnitude smaller than the beam emittance and is thus negligible.

\section{KF Cavities}

At this point, we can perform a similar analysis to estimate the effect of wakefields in the RF cavities. The NLC damping ring will need roughly one to two meters of $1.4 \mathrm{GHz} R \mathrm{RF}^{\prime}$ cavity to provicle sufficient longitudinal acceleration and bunching. To estimate the transverse wakefield, we could simply scale the $11.4 \mathrm{GHz}$ NLC linac wakefield, plotted in Fig. 51, to this lower frequency, but this yields a wakefield that is small since the irises in the $11.4 \mathrm{GHz}$ NLC structure are relatively large.

Instead, we will use the transverse wakefield of the RF cavities in the PEP storage ring, scaled to the $1.4 \mathrm{GHz}$ frequency. ${ }^{[15]}$ Over the range of interest, we can approximate this dipole waliefield as a linearly increasing function of $\Delta z$ :

$$
W_{\perp 1}(\Delta z)=\Delta z 0.12 \mathrm{~V} / \mathrm{pC} / \mathrm{cm}^{3}
$$

Now, we follow the procedure outlined in the previous section: we find the deflection by convolving the wake function over the bunch distribution and then we expand in a Taylor series about $z=0$ to find the coefficients $g_{n}$. Assuming a gaussian distribution, this yields

$$
\begin{aligned}
& y_{1}=-\frac{N e y_{c}}{E_{0}[\mathrm{eV}]} \frac{W_{\perp}^{\prime}}{2} \\
& y_{2}=\frac{N \epsilon y_{c}}{E_{0}[\mathrm{eV}]} \frac{W_{\perp}^{\prime}}{2 \sqrt{2 \pi} \sigma_{z}} \\
& y_{3}=0,
\end{aligned}
$$

where $E_{0}$ is the ring energy in $\mathrm{C} V$ and $\mathrm{W}_{\perp}^{\prime}$ is the slope of the wakefield in $\mathrm{V} / \mathrm{C} / \mathrm{m}^{3}$; 
for the scaled PEP cavity: $W_{\perp}^{\prime}=0.12 \times 10^{18} \mathrm{~V} / \mathrm{C} / \mathrm{m}^{3}$.

Finally, we can calculate the increase in the projected vertical emittance using Eqs. (2.2.84) and (2.2.86). For a worst case estimate, we will assume that all of the cavities are lumped together with the same trajectory offset. In this case,

$$
\left|S_{ \pm}^{(n)}\right|^{2}=g_{n}^{2} \beta_{y} L_{\text {cav }}^{2}
$$

where $L_{\text {cav }}$ is the total length of the RF cavities. In the NLC damping ring, with $2 \times 10^{10}$ particle per bunch, a $100 \mu \mathrm{m}$ orbit offset, and 2 meters of cavity, we find:

$$
\frac{\left\langle y^{2}\right\rangle}{\beta_{y}} \sim 6 \times 10^{-15} \mathrm{~m}-\mathrm{rad} .
$$

While this is much larger than the aflect of the resistive wall wakefield, it is still three orders of magnitude smaller than the vertical emittance and is thus negligible.

\subsubsection{Transverse Quadrupole Wakefield}

The wakefields also have quadrupole and skew quadrupole gradients. But, the quadrupole wakefield tends to be much smaller than the dipole wakefield. One can estimate the importance of this waliefield since the effect of the transverse wakefield roughly scales as $(r / b)^{n}$, where $r$ is the of'set, $b$ is the chamber radius, and $n$ is the field harmonic. Thus, provided that the beam is much smaller than the vacuum chamber radius, the higher-order walefields will be much smaller than the dipole wakefield.

\subsubsection{SUMMARY}

In this section, we have calculated the increase in the projected vertical emittance due to transverse waliefields. In the NLC damping ring, the effect of the low-order coupling on a damped beam is small and the effect of the higher-order coupling should be even smaller. We have not estimated the effect of the longitudinal wakefields nor have we considered all sources of the uransverse wakefield; even though our initial estimates indicate that the wakefields will not present a limitation, these calculations should be perforned. In addition, we have neglected all of the multi-bunch analogs of the single bunch dilutions; in the NLC damping ring, these also need to be estimated. 


\subsubsection{Wakefields: Dynamical Effects}

The wakefields can also lead to dynamical instability. These are usually referred to as coherent instabilities. ${ }^{[20]}$ In general, the instability thresholds do not depend upon the magnitude of the vertical emitiance. Instead, the thresholds depend upon the beam current, the impedance of the ring, and the tune spreads. Thus, these effects do not directly limit the vertical emittance.

However, the wakefields do change the beam response function. We will mention two important effects: first, in a ring with positive chromaticity, the rigid dipole oscillations can be damped; this is referred to as the head-tail damping and can be an important source of damping for coherent oscillations. Second, even though the beam may be stable, interference between the normal modes of oscillation can cause a transient growth in a beam or, more importantly, in a train of bunches. ${ }^{[16)}$ This later effect is important because it will amplify the effect of noise that drives coherent oscillations of the beam; we will discuss these effects further in Section 2.4.

\subsubsection{Beam-Gas Scattering}

In this section, we will determine limitations on the vertical emittance due to beam-gas scattering. Beam-gas scattering occurs when particles in the beam scatter, elastically or inelastically, with the residual gas in the vacuum chamber. In an $e^{+} / e^{-}$ storage ring, the background gas is primarily due to gas desorption occurring when the synchrotron photons hit the vacuum chamber wall. Typically, the residual gas is composed of light molecules such as hydrogen, methane, water vapor and carbon monoxide and dioxide. In addition to the effect on the vertical emittance, beam-gas scattering causes particle losses, and it will ionize the gas which can then cause beam instabilities; we discuss these effects in Sections 2.3.7 and 2.3.2.

We can divide the beam-gas scattering into three processes: elastic scattering with the nuclei, elastic scattering with the atomic electrons, and inelastic scattering with both the nuclei and the atomic electrons. Elastic scattering with a nucleus will deflect the incident particle without significantly changing the particle's energy. In contrast, elastic scattering with the atomic electrons both deflects the incident particle and reduces the particle's energy. Finally, the inelastic scattering, namely bremsstrahlung, causes the circulating particle to lose energy; we can neglect the opening angle of the radiation. 
All of these processes are analogous to the quantum excitation due to synchrotron radiation; they cause a discrete change in the particle's betatron oscillation amplitude. The processes differ from the synchrotron radiation effects in that the beam-gas scattering events occur very infrequently, but the expected amplitude change due to a beam-gas scattering event is much greater than that resulting from the emission of a synchrotron photon. Thus, we will find that for reasonable vacuum pressures, the beam-gas scattering does not affect the core emittance; only a few particles are scattered to significant amplitudes. Instead, the beam-gas scattering will cause a halo of large amplitude particles around the core of the beam.

\subsubsection{Elastic - NUClei}

We are interested in scattering with small momentum transfers; large momentum transfers will cause the particle to be lost. In this regime, the screening effects of the atomic electrons are important; the screening will reduce the number of very small angie collisions. The Born approximation with the Fermi-Thomas model for atomic potential yields a differential cross section of ${ }^{[60,65]}$

$$
\frac{d \sigma}{d \Omega} \approx\left(\frac{2 Z_{r_{0}}}{\gamma}\right)^{2} \frac{1}{\left(\theta^{2}+\theta_{\min }^{2}\right)^{2}}
$$

Here, $r_{0}$ is the classical electron radius, $Z$ is the atomic number, and $\theta_{\min }$ is a function of the screening: $\theta_{\min } \approx \hbar / p a$ where $p$ is the incident particle mumentum and $a$ is the atomic radius: $a \approx 1.4 \pi^{2} / m^{2} Z^{1 / 3}$. We can integrate this to find the total cross section

$$
\sigma \approx 4 \pi Z^{4 / 3}\left(192 r_{0}\right)^{2}
$$

At this point, we can solve for the contribution to the rms equilibrium emittance in the same manner that we calculated the emittance contribution from the opening angle of the synchrotron radiation, Section 2.2.3. The normalized equilibrium emittance is

$$
\gamma_{y}=\gamma \frac{\tau_{y}}{4} \overline{\mathcal{N}}\left\langle 0_{y}^{2}\right\rangle \beta_{y}
$$

where $\left\langle\theta^{2}\right\rangle$ is the expected value of $0^{2} . .<$ is the rate of scattering, and the bar 
denotes the average around the ring. In particular,

$$
\mathcal{N}\left\langle\theta_{y}^{2}\right\rangle=c n_{\text {gas }} \int_{0}^{\theta_{\text {max }}} \frac{d \sigma}{d \Omega} \pi \theta^{3} d \theta
$$

where $c$ is the speed of light, $\theta_{\max }$ is the maximum scattering angle, this is usually determined by the aperture of the machine, and $n_{\text {gas }}$ is the atomic density of the gas; numerically, $n_{\text {gas }}$ is equal to: $n_{\text {gas }}=3.21 \times 10^{22} \mathrm{~mol} . /$ Torr $\mathrm{m}^{3}$ at a temperature of $300^{\circ} \mathrm{K}$.

This yields an equilibrium emittance of

$$
\gamma \epsilon_{y} \approx \pi c r_{y} \frac{Z^{2} r_{0}^{2}}{\gamma}\left[\ln \left(\frac{\theta_{\max }}{\theta_{\min }}\right)-\frac{1}{2}\right] \overline{\beta_{y} n_{\mathrm{gas}}} .
$$

In the NLC darnping ring, $E q .(2.3 .37)$ predicts an emittance equal to the design emittance of $\gamma \epsilon_{y}=3 \times 10^{-8} \mathrm{~m}-\mathrm{rad}$ at a pressure of roughly $10^{-7}$ Torr, assuming $Z=7.3$ and two atoms per molecule; this approximates air. For comparison, typical vacuum pressures are rouglıly $10^{-8}$ to $10^{-9}$ Torr.

Next, we need to examine the particle distribution due to this scattering. We will describe the distribution in terms of the single particle action $J$ which is a quadratic function of the transverse position and momentum and, when averaged over the beam, $J$ is equal to the beam emittance. Synchrotron radiation generates an exponential ciistribution

$$
P_{\mathrm{SR}}(J)=\frac{e^{-J / \epsilon_{y}}}{\epsilon_{y}}
$$

This occurs since many photons are radiated within a damping time. Thus, using the Central Limit Theorem, the clistribution should be gaussian in position and momentum and exponential in $J$. In contrast, the expected number of elastic beamgas collisions is typically' very' small. For example, at a pressure of $10^{-8}$ Torr, we expect roughly ten elastic collisions per second; the damping time in the NLC damping ring is $4 \mathrm{~ms}$. Obviously, the central limit theorem does not apply in this case and thus we cannot expect an exponential distribution in $J$.

Instead, the distribution is dominated by single scattering events. Examining Eq. (2.3.33), we would expect the distribution density in $J$ to depend upon $J^{-2}$ at 
large amplitudes. We can approximate this with a distribution density of the form

$$
P(J) \approx \frac{J_{\min }}{\left(J_{\min }+J\right)^{2}} \quad \text { for } \quad J<J_{\max },
$$

where $J_{\max }$, like $\theta_{\max }$, is determined by the machine aperture and $J_{\min }$ is determined from the requirement that the expected value of $J$ equal the equilibrium emittance due to beam gas scattering, Eq. (2.3.37). Thus,

$$
J_{\min } \approx \frac{\epsilon_{y}}{\ln \left(J_{\max } / \epsilon_{y}\right)}
$$

where we have assumed that $J_{\max } \gg J_{\min }, \epsilon_{y}$.

This distribution due to beam gas scattering decreases relatively slowly with amplitude. Thus, the scattering causes a halo of large amplitude particles which will increase the rms emittance even though the core of the beam is relatively unaffected. For example, in the NLC damping ring, a vacuum pressure of $10^{-7}$ Torr will double the rms equilibrium emittance. But, if one neglects the outer $3 \%$ of the beam, one finds that the emittance increase due to the scattering is only $10 \%$. Of course, in a linear collider, these large amplitucle particles must be collimated or they will cause large background signals at the detector.

This is illustrated in Fig. 17. The solid line is the calculated distribution $P\left(J_{y}\right)$, due to both synchrotron radiation and beam-gas scattering, when the background pressure is $10^{-7}$ Torr. In such a case, the expected value $\left\langle J_{y}\right\rangle$ is twice that due to the synchrotron radiation alone. The dashed line is the distribution $P_{S R}\left(J_{y}\right)$. One can see that the two distributions are extremely similar for values $J_{y} \lesssim 6\left\langle J_{S R}\right\rangle$ and, although they differ significantly for larger values of $J_{y}$, there are very few particles in these large amplitude tails.

\subsubsection{Elastic - EleCtrons}

Now, we can consider elastic scattering with the atomic electrons. In contrast to elastic scattering with a nucleus, elastic scattering events with the atomic electrons cause both an angular deflection and an energy transfer. Again, we are only interested in small momentum transfers since hard scatterings will cause particle losses. 


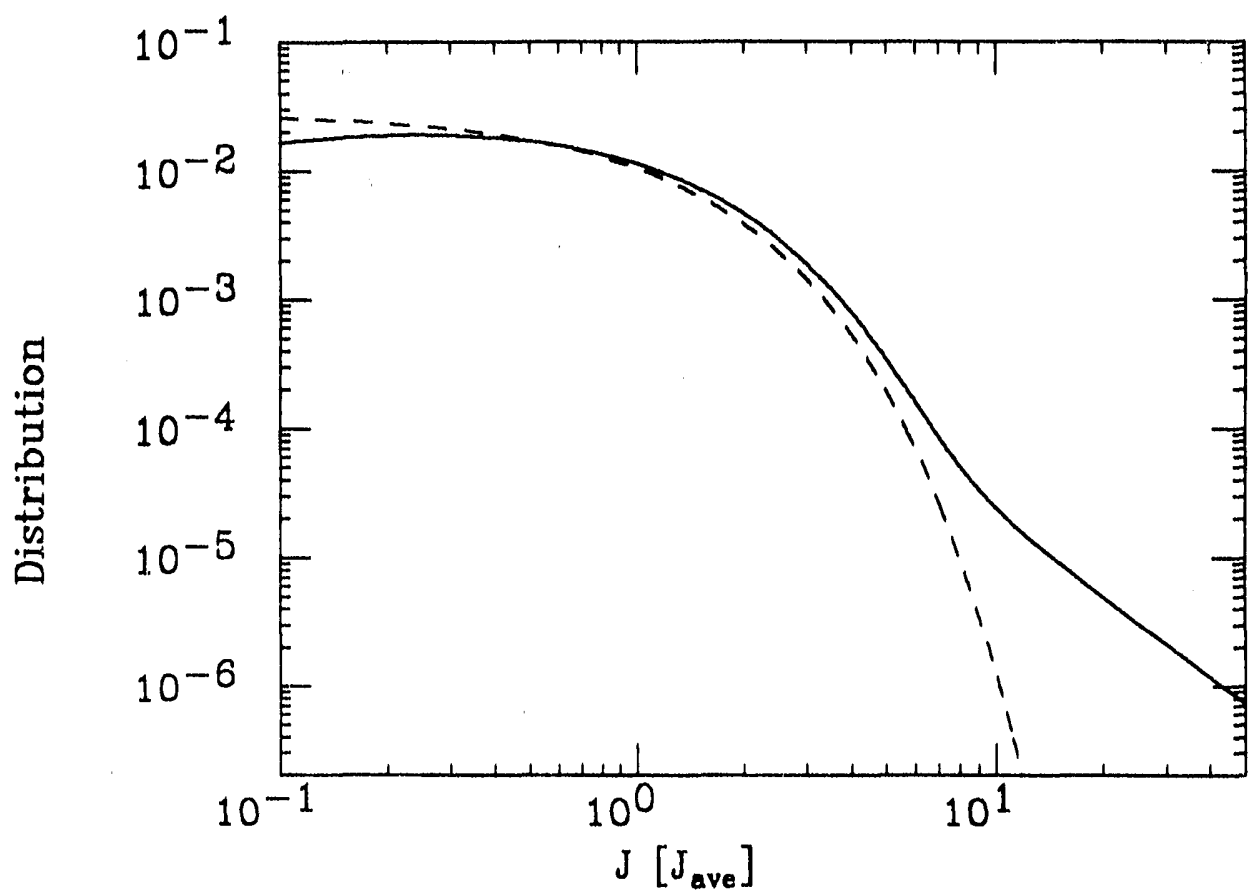

Fig. 17. The effect of beam-gas scattering on the beam distribution in the NLC damping ring with a background pressure of $10^{-7}$ Torr; the solid line is the distribution with beam-gas scattering while the dashed line only includes the effect of the synchrotron radiation.

In this regime, the differential cross section is ${ }^{[16]}$

$$
\frac{d \sigma}{d \delta}=\frac{2 \pi r_{0}^{2}}{\gamma} \frac{1}{\delta^{2}}
$$

where $\delta$ is the relative energy loss: $\delta=\Delta E / E$. Alternately, we could express this in terms of the scattering angle $\theta=\sqrt{2 \delta / \gamma}$. In this case, we would find a cross section equal to Eq. (2.3.33) with the substitution $Z \rightarrow 1$.

Now, we can calculate the resulting equilibrium emittance. The energy loss is coupled to the betatron amplitude via the dispersion function: $\Delta y_{\beta}=\eta_{y} \delta$ and $\Delta y_{\beta}^{\prime}=\eta_{y}^{\prime} \delta$, while the angular kick directly changes $y_{\beta}^{\prime}$. Thus, these two effects are uncorrelated and we can simply add the emittance contributions. Since the angular deflection by the elcctron is similar to that given by a nucleus, the emittance contribution is given by Eq. (2.3.37) with the substitution $Z^{2} \rightarrow Z$. In addition, the amplitude limit $0_{\max }$ is now the lesser of the limit due to the angular deflection or the limit imposed by the energy change. For typical damping ring parameters, these two limits are comparable and thus we will neglect this distinction. Thus, we 
find an emittance contribution due the angular kicks of

$$
\gamma \epsilon_{y} \approx \pi c \tau_{y} \frac{Z r_{0}^{2}}{\gamma}\left[\ln \left(\frac{\theta_{\max }}{\theta_{\min }}\right)-\frac{1}{2}\right] \overline{\beta_{y} n_{\mathrm{gas}}} .
$$

In air this contribution is roughly $15 \%$ of the contribution from the elastic scattering with the nuclei.

Next, calculating the emittance contribution from the energy change is completely analogous to calculating the equilibrium emittance due to synchrotron radiation. Here, the emittance contribution is

$$
\gamma \epsilon_{y}=\gamma \frac{\tau_{y}}{4} \overline{\mathcal{N}\left\langle\delta^{2}\right\rangle \mathcal{H}_{y}}
$$

where $\mathcal{H}_{y}$ is a function of the dispersion and it's derivative, Eq. (A.5.9). The value of $\mathcal{N}\left\langle\delta^{2}\right\rangle$ is found from the cross section Eq. (2.3.41):

$$
\mathcal{N}\left\langle\delta^{2}\right\rangle=c n_{\text {gas }} \int_{0}^{\delta_{\max }} \delta^{2} \frac{d \sigma}{d \delta} d \delta
$$

where $\delta_{\max }$ is determined by the aperture of the storage ring; typically, this is limited to a few percent. We find an emittance contribution of

$$
\gamma \epsilon_{y} \approx 2 \pi c \tau_{y} Z r_{0}^{2} \delta_{\max } \overline{\mathcal{H}_{y} n_{\text {gas }}}
$$

In the NLC damping ring, this contribution is much smaller than the contribution from the angular deflection, Eq. (2.3.42), and thus we will neglect it.

\subsubsection{Inelastic Scattering}

Finally, we consider the inelastic scattering of the beam particles with the residual gas. In this case, bremsstrahlung photons are emitted causing the incident particle to lose energy. We are only interested in small energy changes, less than a few percent of the incident particle energy. In this regime, the differential cross section for scattering with both the nucleus and the atomic electrons is ${ }^{[16]}$

$$
\frac{d \sigma}{d \delta} \approx \frac{16}{3} \frac{Z(Z+1.35)}{137} \frac{r_{0}^{2}}{\delta} \ln \left(\frac{183}{Z^{1 / 3}}\right) .
$$

Now, we can calculate the equilibrium emittance due to these bremsstrahlung 
photons using Eq. (2.3.43). This yields an equilibrium emittance of

$$
\gamma \epsilon_{y} \approx \gamma \frac{2 c \tau_{y}}{3} \frac{Z(Z+1.35)}{137} r_{0}^{2} \delta_{\max }^{2} \ln \left(\frac{183}{Z^{1 / 3}}\right) \overline{\mathcal{H}_{y} n_{\text {gas }}}
$$

In the NLC damping ring with a pressure of $10^{-8}$ Torr, the bremsstrahlung induced emittance is many orders of magnitude less than the elastic scattering contribution, and thus we can ignore it.

\subsubsection{SUMMARY}

In the low energy damping rings, we have found that the most important beam gas contribution comes from the elastic scattering with the gas nuclei. In the NLC damping ring, a pressure of $10^{-8}$ Torr will cause a $10 \%$ vertical rms emittance increase. But, it is important to realize that this emittance increase is dominated by a few large scattering events; the core of the beam is virtually unaffected while population of the tails of the beam distribution is increased slightly. Thus, we conclude that the beam gas scattering is not a significant limitation, even at much higher vacuum pressures.

\subsubsection{Lifetimes}

In a damping ring, it is unlikely that the beam lifetimes could limit the operation of the ring since the beams are stored for a very short time. But, poor beam lifetimes could make commissioning and studying the ring difficult. The primary beam lifetime limitations are excessively small aperture, beam-gas scattering, and the Touschek effect; the later refers to large angle collisions between particles within the beam that lead to particle loss.

With adequate design and tolerances, one should be able to avoid the first limitation. In addition, with reasonable vacuum pressures, the lifetime due to beamgas scattering should not be significant. Formulas for the beam gas lifetimes are listed in Ref. 68. Assuming a pressure of $10^{-8}$ Torr in the NLC damping ring, the lifetime due to beam gas scattcring is roughly four hours; this is more than sufficient.

In contrast, the Touschek lifetime will tend to be more severe since it depends inversely upon the particle density in the beam. In the limit of non-relativistic 


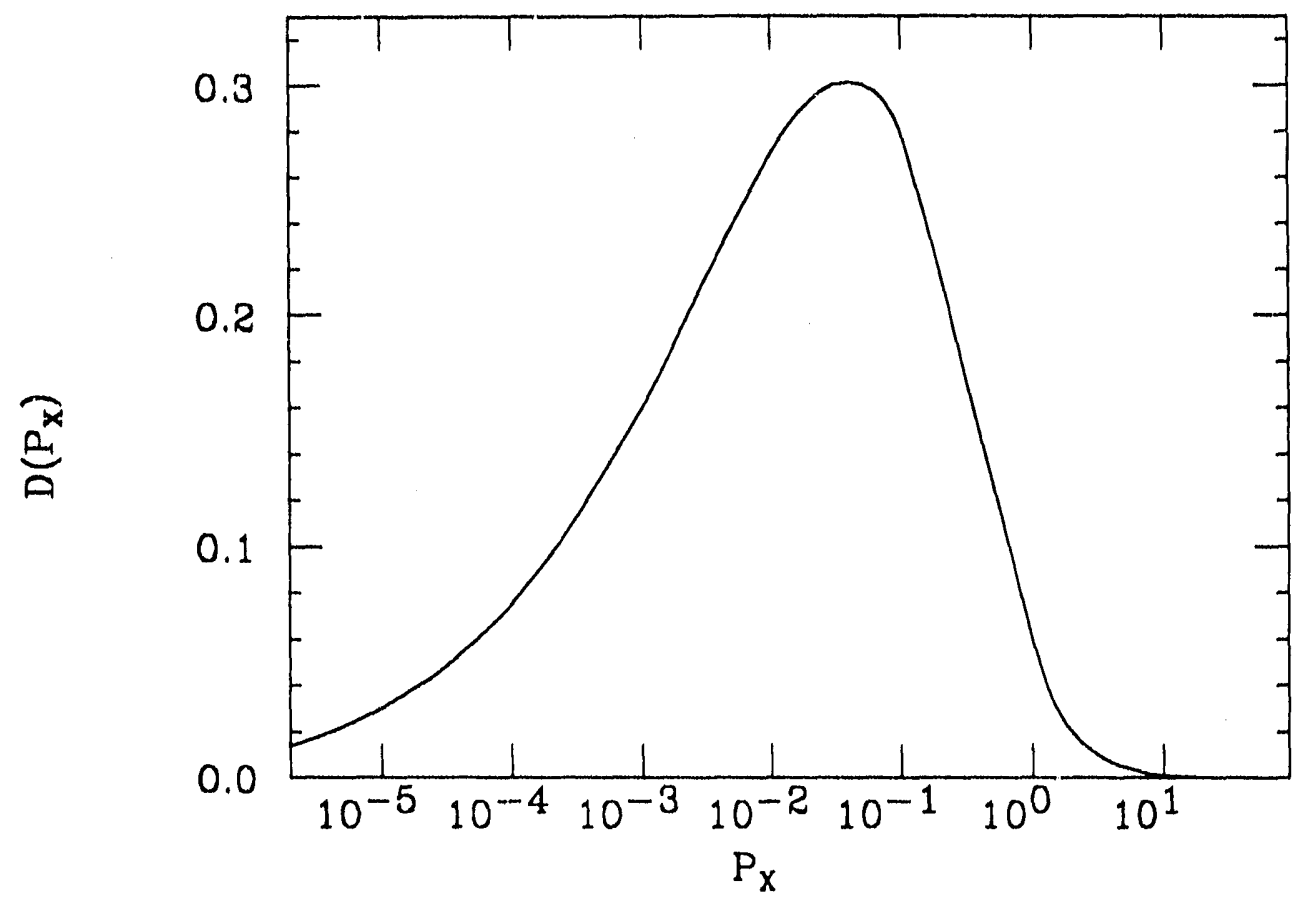

Fig. 18. The function $D\left(P_{x}\right)$.

transverse momenta, the Touschek lifetime is given by ${ }^{[24,4,124]}$

$$
\frac{1}{\tau_{\text {tou }}}=\frac{N r_{\tilde{g}}^{2} c D\left(P_{x}\right)}{8 \pi \sigma_{x} \sigma_{y} \sigma_{z} \gamma^{2} \delta_{\max }^{3}}
$$

where $P_{x}=\delta_{\max }^{2} \beta_{x} / 2 \gamma^{2} \epsilon_{x}$ and $D\left(P_{x}\right)$ is plotted in Fig. 18 ; for typical parameters $D\left(P_{x}\right)$ is between 0.1 and 0.3 . In the NLC damping ring, the Touschek lifetinue is roughly two minutes. While this is long compared to the operating beam storage time of $28 \mathrm{~ms}$, it may be too short to study the properties of a stored beam. In this case, we can increase the vertical beam size or clecrease the number of particles per bunch, thereby decreasing the beam density and increasing the lifetime. 


\section{CHAPTER 2.4 \\ JITTER AND NOISE EFFECTS}

In this chapter, we will discuss the effect of slow and fast jitter processes that can increase the effective emittance of the beam. The sources can be divided into low frequency contributions, such as ground motion and power supply fluctuations, where the jitter adiabatically changes the ring orbit and high frequency effects which drive coherent oscillations. We will estimate the magnitude of these effects and then briefly mention some of the feedback techniques that will almost certainly be needed for compensation.

\subsubsection{Ground Motion}

The ground has many frequencies of movement with periods ranging from years to fractions of a second. Here, we will discuss the "fast" motion where the ground vibrates at the micron level with frequencies between $0.1 \mathrm{~Hz} \lesssim f \lesssim 100 \mathrm{~Hz}^{[42]}$ the high frequency end of this spectrum is primarily due to cultural noise, i.e., manmade noise, while the low frequency vibrations are primarily nature.

This ground motion moves the quadrupole magnets which then deflect the closed orbit. In general, the vibration has a small effect, unless the wavelength is smaller than or comparable to the betatron wavelength in the ring. ${ }^{[4,04]}$ Thus, the ground motion tends to be less of a problem in small strong focusing rings than in the large rings such as LEP, HERA, and the SSC.

At the SLAC and DESY sites, the measured ground motion near the surface has a phase velocity of $250 \sim 300 \mathrm{~m} / \mathrm{s}$ and, during the day, the rms amplitude is roughly $0.2 \mu \mathrm{m} ;{ }^{[12,85]}$ during the night, the amplitude is much less. Finally, the noise has a power spectrum that tends to decay as $1 / f^{2}$. Note that the phase velocity of these high frequency ground waves is roughly an order of magnitude slower than that of the long vavelength motion measured during seismic events. The longer wavelengths sample deeper and denser material and thus have faster velocities. ${ }^{[3]}$

In the NLC damping ring, the average vertical betatron wavelength is roughly $C / \nu_{y}=14 \mathrm{~m}$. Using the results of Refs. 4 and 94 , the first resonant frequency occurs at approximately $20 \mathrm{~Hz}$. Although this is at the high frequency end of the power spectrum, it still suggests that the ground motion might be a problem in the NLC ring; a more detailed analysis, including the actual ring optics and the response of the magnet supports, is needed to determine the real magnitude of the problem. 
If indeed the ground motion is a problem, there are three solutions: (1) choosing a site with reduced noise, much of the high frequency noise is caused by motor vehicle traffic, (2) use isolation supports to damp the noise, and (3) use low frequency feedback systems to stabilize the closed orbit; this will be discussed later.

\subsubsection{Power Supply Fluctuations}

In general, the magnet fluctuations due to power supply fluctuations are low frequency $f \lessgtr 1 \mathrm{KHz}$ in comparison to the beam revolution frequency; higher frequency fluctuations are attenuated by the vacuum chamber. Thus, the fluctuations cause adiabatic changes. Fluctuations of the correctors change the closed orbit and fluctuations of the quadrupoles change both the betation tunes and the closed orbit. In addition, fluctuations of the bending magnets cause the ring energy to change; this occurs because, in an $e^{+} / e^{-}$storage ring, the RF fixes the revolution period.

\section{Correctors}

The dipole correctors usually have independent power supplies and we assume that the fluctuations are independent. Thus, using Eq. (2.2.13), we find a tolerance

$$
\Delta 0_{y \text { rms }} \lesssim \frac{\sigma_{y} 2 \sqrt{2}\left|\sin \pi \nu_{y}\right|}{\sqrt{\Lambda_{\text {corr }}} \beta_{y}},
$$

where $N_{\text {corr }}$ is the number of corrector's and $\beta_{y}$ is the vertical beta function at the correctors.

In the NLC damping ring design, this yields a tolerance of $\Delta \theta_{y} \mathrm{rms} \lesssim 0.08 \mu \mathrm{rad}$ to limit the jitter to one quarter of the vertical beam size. We can estimate the maximum required corrector strength from the alignment tolerances and the magnet strengths; a maximum deflection of $1 \mathrm{mrad}$ should be more than sufficient. This

yields a relative tolerance on the corrector power supplies of $\Delta I / I_{\text {peak }} \lesssim 8 \times 10^{-5}$. This is a fairly tight tolerance, but it could be eased by reducing the maximum corrector strengths.

\section{Main Bending Magnets}

We will assume that all of the bending dipoles are powered by the same supply. In this case, slow fluctuations of the dipoles will change the beam energy; as mentioned, this occurs because the revolution period is fixed by the RF in an $e^{+} / e^{-}$ ring. In terms of the closed orbit and the betatron tunes, slowly changing the main 
bends is equivalent to changing the correctors and the quadrupoles in the opposite direction. Thus, in the vertical plane, the closed orbit change due to the fluctuation can be written

$$
\Delta y_{c}=\eta_{y}(s) \frac{\Delta B}{B}
$$

This can be further simplified since, in Section 2.2.4, we saw that $\sigma_{y} \sim 2 \eta_{y} \sigma_{c}$. Thus, to limit the orbit variation to one quarter of the vertical beam size, we find a tolerance

$$
\frac{\Delta B}{B} \lesssim \frac{\sigma_{\epsilon}}{2}
$$

In the NLC damping ring this tolerance is roughly $5 \times 10^{-4}$.

In addition to the closed orbit, the betatron tunes will vary as the bends change. As mentioned, changing the bends is equivalent to changing the quadrupoles in the opposite direction. Thus, the tune shift is

$$
\Delta \nu=\xi_{\text {uncor }} \frac{\Delta B}{B}
$$

where $\xi_{\text {uncor }}$ is the uncorrctcd clromaticity. Notice that the sextupoles do not compensate this "chromatic" tune fluctuation. This occurs because the horizontal orbit change is not proportional to the horizontal dispersion since the revolution period is fixed. In the NLC damping ring, the uncorrected chromaticity is roughly -25 . Thus, to limit the tune fluctuation to 0.01 , we find a iolerance of

$$
\frac{\Delta B}{B} \lesssim 4 \times 11^{-4}
$$

This is comparable to the tolerance on the closed orbit fluctuations and should not be difficult to achieve; commercial power supplies are available with regulation of $10^{-4} \sim 10^{-5}$.

Quadrupoles

In this case, we will assume that all of the focusing quadrupole magnets are powered by a single supply, while all of the defocusing quadrupoles are powered by another supply. First, we can solve for the vertical orbit change with Eq. (2.2.13). Here, the deflection of the closed orbit depends upon the quadrupole field and the closed orbit offset. Although the strength variation is correlated between magnets, 
the orbit offsets of a corrected orbit are not. Thus, we find a result analogous to Eq. (2.4.1):

$$
\Delta \Pi_{1} \lesssim \frac{\sigma_{y} 2 \sqrt{2}\left|\sin \pi \nu_{y}\right|}{L_{\text {quad }} y_{c \text { rms }} \sqrt{N_{\text {quad }}} \beta_{y}},
$$

where $L_{\text {quad }}$ is the length of the quadrupole, $y_{c}$ is the rms magnitude of the closed orbit, and $\Delta K_{1}$ is the rms error in the normalized quadrupole gradient.

Finally, we can estimate the tune variation due to the quadrupole fluctuations. If we wish to limit this to $\Delta \nu \lesssim 0.01$, we find a to'erance

$$
\frac{\Delta K_{1}}{\Gamma_{1}} \lesssim \frac{0.01}{\xi_{u n c o r}}
$$

where $\xi_{\text {uncor }}$ is the uncorrected chromaticity.

In the NLC damping ring design, the closed orbit constraint imposes power supply tolerances of $\Delta I / I \sim 2 \times 10^{-4}$ to limit the jitter to one quarter of the beam size. Here, we have assumed 100 quadrupoles with $K=20 \mathrm{~m}^{-2}, L=0.2 \mathrm{~m}$, and $y_{c}=100 \mu \mathrm{m}$; these values should overestimate the severity of the tolerance. Similarly, the tune variation imposes a comparable tolerance of $\Delta I / I \lesssim 4 \times 10^{-4}$. In both cases, commercially available supplies should be able to meet these tolerances.

\subsubsection{High Frequency Jitter}

The primary sources of high frequency noise are the RF system, the high frequency feedback systems that would likely be needed to damp coherent oscillations, and the injection/extraction kickers. The noise will drive coherent oscillations of the beam. Longitudinal noise, such as that from the RF system, will drive coherent synchrotron oscillations that, in the presence of synchro-betatron coupling, will cause coherent betatron oscillations. Transverse noise will directly drive coherent transverse oscillations.

A coherent betatron oscillation can be damped by the head-tail damping process, synchrotron radiation damping, and feedback systems. In addition, if there is a large spread in the oscillation tune, the coherent oscillation can filament before it is damped coherently; the oscillation decoheres and the beam size increases. After this filamentation, the fast head-tail process and feedback cannot damp the beam. Thus, the filamented beam damps at the (slower) synchrotron radiation damping rate. 
We can estimate a tolerance on the RF noise in the same manner that we calculate the equilibrium emittance. Assume that the noise causes a discrete transverse deflection $\theta$ that is uncorrelated between revolutions. Then, the change per turn of the single particle invariant that describes the coherent oscillation is $2 \Delta J=\beta \theta^{2}$. By equating this with the damping rate, we find the expected value of $\langle J\rangle$ :

$$
\langle J\rangle=\frac{\beta\left\langle\theta^{2}\right\rangle}{4} \frac{\tau}{T_{0}},
$$

where $\tau$ is the damping time and $T_{0}$ is the period of revolution.

To prevent dilution of the effective emittance, we require $\langle J\rangle\langle\epsilon$. For a worst case estimate, we can assume that the beam damps at the synchrotron radiation damping rate. Thus, in the $\mathrm{NLC}$ damping ring design, we find a tolerance on the vertical deflecting voltage

$$
V_{y \text { rnis }} \lesssim 20 \text { Volts }
$$

to limit the jitter to one fourth the beam size. This can be translated into a tolerance on the phase stability of the main accelerating RF:

$$
\epsilon_{y} Z\left\langle\phi^{2}\right\rangle \mathcal{H}_{y}\left(\frac{V_{\mathrm{RF}}}{E_{0}}\right)^{2} \frac{\tau}{4 T_{0}}
$$

In the NLC damping ring design. assuming a 1 MV RF system with $1 \mathrm{~mm}$ of vertical dispersion in the cavities. this implies a phase stability of $\phi_{\mathrm{rms}} \sim 2^{\circ}$ which is a relatively loose tolerance.

Finally, we should note that a more severe tolerance on the RF phase is due to the phase stability required of the extracted beam. Phase jitter of the extracted beam is translated into energy jitter at the beginning of the bunch compressor; a correlated energy spread is added to the bunch at the beginning of the bunch compressor using an acceleration section, phased to give the nominal center of the bunch zero energy gain. 'This energy jitter is then translated back into phase jitter during the compression, since low and high energy particles have different path lengths. Finally, the phase jitter is translated into an energy jitter in the linac. This effect has been observed in the SLC' but we will not calculate tolerances here since it requires detailed knowledgre of the bunch compressors. 


\subsubsection{Feedback}

Stabilization of the closed orbit is very important in synchrotron light sources. Currently, many of the synchrotron light sources stabilize the closed orbit at the level of a few microns. ${ }^{(34,63,76,126)}$ The primary limitation of these feedback systems is the measurement of the beam position. This is a problem that needs to be resolved throughout the linear collider complex; one needs to accurately measure the beam position at the level of the beam size. But, assuming that the required measurement accuracy can be achieved, stabilization of the closed orbit should be straight-forward.

In addition, to slow feedback for the closed orbit, fast feedback can be used to damp coherent oscillations of the beam. Wide band bunch-to-bunch feedback systems are used to stabilize the beam in PETRA and HERA ${ }^{(41,52)}$ and are planned for in the LBL-LLNL-SLAC B-Factory design. ${ }^{[21,132]}$ In the NLC damping ring, one may not be able to feedback on each bunch, this requires a 1-2 GHz feedback system, but it should be relatively simple to feedback on the batches of ten bunches; this only requires a $20 \mathrm{MHz}$ system which is comparable to the PETRA feedback system. Of course, again, any feedback system will be limited by the resolution of the beam measurement. 


\section{CHAPTER $\quad 2.5$ \\ INJECTION MATCHING}

In a damping ring, it is important to match the injected beam to the ring. In the transverse case, the beam must be injected onto the closed orbit, and the beta functions and the dispersion must be matched correctly. If there is a mismatch, the beam will filament; the beam is large at injection and thus nonlinear fields cause a significant tune spread. The filamentation of the mismatch will increase the beam size and thereby increase the storage time needed to damp the beam to the equilibrium value.

The emittance dilution due to filamentation of a beta mismatch can be written: ${ }^{[35]}$

$$
\frac{\Delta \epsilon}{\epsilon}=\frac{1}{2}\left(\frac{\beta}{\beta^{\star}}+\frac{\beta^{\star}}{\beta}\right)+\frac{1}{2}\left(\alpha^{\star} \sqrt{\frac{\beta}{\beta^{\star}}}-\alpha \sqrt{\frac{\beta^{\star}}{\beta}}\right)^{2}-1
$$

where $\alpha$ and $\beta$ are the machine parameters and $\alpha^{\star}$ and $\beta^{\star}$ are the beam parameters. Similarly, the dilution due to a filamented dispersion mismatch is ${ }^{[73]}$

$$
\Delta \epsilon=\frac{\mathcal{H} \sigma_{\epsilon}^{2}}{2}
$$

where $\mathcal{H}$ is the dispersion function defined in Eq. (A.5.9). Finally, the dilution due to a orbit mismatch is ${ }^{(110)}$

$$
\Delta \epsilon_{y}=\frac{1}{2}\left(\gamma_{y} y_{0}^{2}+2 \alpha_{y} y y_{0}^{\prime}+\beta_{y} y_{0}^{\prime 2}\right)
$$

where $y_{0}$ and $y_{0}^{\prime}$ are the difference between the closed orbit and the injected trajectory.

In addition to the transverse matching, the beam must be matched longitudinally to prevent coherent synchrotron oscillations. The synchro-betatron coupling could couple the synchrotron oscillations to the transverse planes as discussed in section 2.2.6. This increases the beam size when the oscillations filament. The nonlinear synchro-betatron coupling is more important at injection since the beam occupies a large portion of the accelerating RF and thus experiences the sinusoidal variation of the fields. Furthermore, since the bunch is usually longer, the transverse wakefields, which are an important source of coupling, are much stronger. These effects can be estimated from the results of Sections 2.2 .6 and 2.3 .4 , although a detailed analysis has not yet been performed for the NLC damping ring. 
Actually, in the NLC damping ring design, the tolerances on the injection mismatch are very loose. This occurs because the beam is over-damped to ease tolerances on the vertical equilibrium emittance. The horizontal and longitudinal emittances are damped to their equilibrium values after roughly half the damping cycle and thus injection errors will have negligible effect on the extracted emittances. Even the vertical emittance, which requires the most damping, is dominated by the equilibrium value at the end of the damping cycle. The injected emittance of $\gamma \epsilon_{y}=3 \times 10^{-3} \mathrm{~m}$-rad contributes roughly $10 \%$ of the extracted emittance of $\gamma \epsilon_{y}=3 \times 10^{-8} \mathrm{~m}$-rad. Thus, even if the injected beam filaments to twice its size, the extracted emittance will only increase by $10 \%$. 


\section{CHAPTER 2.6 GENERATION SUMMARY}

In this chapter, we have discussed effects that can limit the vertical emittance in future damping rings. In particular, we have calculated limitations due to single particle effects, collective effects, and pulse-to-pulse jitter sources. We then applied these results to the current design of the NLC damping ring which calls for a very low vertical emittance of $\gamma \epsilon_{y}=3 \times 10^{-8} \mathrm{~m}$-rad with an aspect ratio $\left(\epsilon_{x} / \epsilon_{y}\right)$ of 100 .

The single particle limitations are primarily due to vertical dispersion and betatron coupling; the more fundamental limitation, due to the opening angle of the synchrotron radiation, is a factor of 45 smaller than the design vertical emittance. The vertical dispersion and the betatron coupling impose alignment tolerances on the ring components. In the NLC damping ring, we have calculated that vertical alignment tolerances of roughly $100 \mu \mathrm{m}$ will limit the vertical emittance to $\gamma \epsilon_{y}<2.5 \times 10^{-8} \mathrm{~m}$-rad with a $95 \%$ confidence level; these results are summarize in Table 11 .

Next, the most important collective effects are intrabeam scattering and the space charge tune shift. The space charge tune shift reduces the working area in turie space. In the current design, the space charge tune shift is $\Delta \nu_{y}=-0.015$. While this is not thought to pose a problem, a larger value might cause the induced synchrotron sidebands to overlap a strong resonance, leading to emittance growth. Of course, for given normalized emittances, the space charge tune shift depends inversely upon $\gamma^{2}$ and thus it decreases rapidly as the ring energy is increased.

In contrast, for given normalized emittances, intrabeam scattering does not necessarily decrease rapidly with the ring energy. But, in the current NLC design, intrabeam scattering only increases the rms vertical emittance by $20 \%$; this only increases to a $120 \%$ dilution as the vertical emittance is decreased to the limit imposed by the opening angle of the synchrotron radiation (a factor of 45). Thus, the intrabeam scattering is "annoying", but it does not present a severe limitation.

Furthermore, the current theories of intrabeam scattering may over-estimate the real emittance growth. These theories estimate the rms emittance, but, with scattering, the distribution becomes non-gaussian and thus the rms emittance does not characterize the beam emittance well. The emittance growth due to beamgas scattering is an example of this. In the NLC damping ring, a background gas pressure of $10^{-7}$ Torr will cause the rms vertical emittance to double. But, the 
actual effect on the beam is small; the scattering generates a halo of large amplitude particles without significantly diluting the core of the beam.

Finally, we have also estimated tolerances on the sources of pulse-to-pulse jitter. In the current design, the tolerances on the power supplies, which limit the beam jitter to $25 \%$ of the rms beam size, are reasonable $\left(\Delta I / I \$ 10^{-4}\right)$. But, tolerances on the ground motion may be more severe; the ring will be resonant to ground motion with frequencies above approximately $20 \mathrm{~Hz}$. Fortunately, in a storage ring, it is relatively simple to stabilize the beam with feedback, easing both the power supply tolerances and the ground motion tolerances.

Thus, to conclude, there does not appear to be any significant limitation in achieving the design vertical emittance of $\gamma \epsilon_{y}=3 \times 10^{-8} \mathrm{~m}-\mathrm{rad}$ in the NLC damping ring. In fact, it seems quite possible that one might be able to reduce this an order of magnitude to $3 \times 10^{-9} \mathrm{~m}$-lad. This would require reducing the alignment tolerances by roughly a factor of four, and, perhaps, increasing the damping ring energy to reduce the space charge tune shift and the opening angle contribution to the emittance. Furthermore, one would need beam position monitors with submicron accuracy for the feedback systems.

Of course, we must qualify these statements by noting that we have neglected two important effects: first, the effect, on the emittance, of multiple closely spaced bunches needs to be considered. The intra-bunch wakefields could cause the bunches in a train to have different trajcctories, increasing the effective emittance of the bunch train. Second, effects that occur at injection, before the beam damps should also be considered. Here, the bunch length and the energy spread are much larger and the nonlinear synchrobetatron resonances might enlarge the initial injected beam; fortunately, as mentioned, this would not be extremely significant in the NLC damping rings since the vertical emittance is over-damped. 
Chapter 3.1

\section{CHAPTER 3 \\ ACCELERATION OF BEAMS WITH SMALL VERTICAL EMITTANCES}

After the beams are generated in the damping rings, they need to be accelerated to the desired final energy while preserving the small beam emittances. In many current designs, as schematically illustrated in Fig. 1, the beams are extracted from the rings into a bunch compression and matching region. Then, the beams are accelerated, in a preliminary linac, to an intermediate energy where they undergo a second bunch compression. Finally, the beams are accelerated in the main linac to the desired energy.

In this chapter, we will discuss effects that can dilute the vertical emittance during the acceleration. We will limit the discussion to the linacs and neglect dilutions that occur in the bunch compressors and other sections of the linear collider. Throughout, we will calculate with parameters of the NLC designs described in Appendix $\mathrm{D}$; note that the linac parameters are still in a state of flux, but those listed in Appendix D provide a representative set.

There are three forms of emittance dilution that can increase the effective vertical emittance in the linacs: conservative dilutions where the six-dimensional emittance is conserved but the projected emittance is increased, pulse-to-pulse jitter where the beam emittance is not necessarily increased, but the effective beam size is c nlarged, and finally, non-conservative dilutions, such as scattering and radiation, that directly increase the beam emittance.

The conservative dilutions are similar to those that lead to a local beam size increase in the damping ring; this was discussed in Section 2.2. These dilutions only increase the projected emittance beam, and, in theory, the effects can be removed. Unfortunately, in a linac, the dilutions "filament" because the beam has a finite energy spread and the phase advance is energy dependent. Thus, after propagating through the linac for a short distance, the dilutions become very difficult to remove and effectively become an emittance increase.

We discuss the equations of motion in a linac and the filamentation process in Sections 3.2 and 3.3. Then, in Section 3.4, we calculate the primary conservative dilutions which are due to clispersive errors, transverse wakefields, and RF deflections; these are analogous to the local dispersive and synchro-betatron coupling in the damping ring. In Sections 3.5 and 3.6, we discuss methods of easing some of 
the tolerances that these dilutions impose, and finally, in Section 3.7, we calculate the effect of magnetic field errors which cause betatron mismatches and transverse betatron coupling.

Next, in Section 3.8, we discuss the effect and sources of pulse-to-pulse jitter; these effects are important in future linear colliders because the beam sizes are very small. Finally, in Section 3.9, we discuss a few additional dilutions, including the beam space charge and the non-conservative dilutions. The non-conservative dilutions are similar to those that determine the beam emittance in the damping rings, but in a linac, these effects are very small.

Throughout this chapter, we will calculate tolerances to limit the vertical emittance dilution to roughly $6 \%$; this causes a $3 \%$ reduction in the luminosity. We choose to limit the individual dilutions to this small value because there are many independent sources of dilution, all of which add to the final result. 
Chapter 3.2 .1

\section{CHAPTER 3.2 \\ EQUATIONS OF MO'TION AND PROJECTED EMITTANCES}

\subsubsection{Equations of Motion}

In a high energy linear accelerator, the longitudinal motion is effectively independent of the transverse motion. There are sources of coupling between the longitudinal and transverse planes, but the longitudinal emittance is much greater than the transverse emittance (between four and six orders of magnitude greater in the NLC linacs) and thus we can neglect the perturbations to the longitudinal plane from the transverse motion.

Furthermore, in a high energy linear accelerator, the longitudinal position is essentially fixed; as discussed in Appendix A, it is independent of the energy deviation as well as the transverse motion. For example, in the NLC main linac, particles within $3 \sigma$ of the design energy and beam divergence shift longitudinal position by much less that $1 \mu \mathrm{m}$; in contrast, the bunch length is roughly $100 \mu \mathrm{m}$.

Since the transverse motion does not affect the longitudinal, we can parameterize the transverse motion with the longitudinal coordinates. The dominant effects in a linear accelerator are due to the focusing and deflecting magnetic fields and the wakefields. Thus, the transverse equations of motion can be written:

$$
\begin{aligned}
& \frac{1}{\gamma(s)} \frac{d}{d s} \gamma(s) \frac{d}{d s} y(s ; z, \delta)-(1-\delta) K_{1}\left[y(s ; z, \delta)-y_{q}\right]=(1-\delta) G_{y} \\
& \quad+\frac{(1-\delta)}{\gamma(s)} N r_{0} \int_{z}^{\infty} d z^{\prime} \int_{-\infty}^{\infty} d \delta^{\prime} \rho\left(z^{\prime}, \delta^{\prime}\right) W_{\perp 1}\left(s ; z^{\prime}-z\right)\left[y\left(s ; z^{\prime}, \delta^{\prime}\right)-y_{a}\right]
\end{aligned}
$$

where the only difference between this equation and that for the motion in a storage ring is the dependence on $\gamma(s)$ which leads to the adiabatic damping.

Specifically, $s$ and $z$ are the longitudinal position in the accelerator and in the bunch, and $\delta$ is the relative energy deviation which is also a function of $s$ and $z$. Next, $K_{1}^{\prime}$ and $G$ are the normalized focusing and bending functions, and $W_{\perp 1}$ is the transverse dipole wakefield which depends upon the dipole moment of the bunch; wakefields are introduced in Section 2.3.4 and the wakefelds in the NLC are plotted in Appendix D. In addition, $N$ and $r_{0}$ are the number of particles and the classical electron radius, $\rho$ is the longitudinal distribution function for the particle 
bunch, and, $y_{q}$ and $y_{a}$ are the misalignments of the quadrupoles and the accelerator structures. Finally, we have neglected skew quadrupole and higher-order multipole fields and the higher-order wakefields; these are discussed in Sections 3.7 and 3.4.2, respectively.

Now, we need the longitudinal equations of motion. As mentioned, the longitudinal position $z$ is fixed; this leaves the relative energy deviation $\delta$. The energy spread consists of two components: an uncorrelated energy spread which comes from the finite longitudinal emittance and all energy spread that is correlated with $z$; this correlated energy spread is due to incomplete bunch compression, the longitudinal wakefields, and the accelerating RF voltage. Neglecting any initial correlation, we can express this as

$$
\delta(s)=\delta_{u n} \frac{\gamma_{0}}{\gamma(s)}+\int_{0}^{s} d s^{\prime} \epsilon \Delta E_{R F}\left(s^{\prime} ; z\right)+\frac{N r_{0}}{\gamma(s)} \int_{0}^{s} d s^{\prime} \int_{z}^{\infty} d z^{\prime} W_{\| 0}\left(s^{\prime} ; z^{\prime}-z\right)
$$

where $\gamma_{0}$ is the initial energy, $\delta_{u n}$ is the initial uncorrelated energy spread, and $W_{\| 0}$ is the lowest order longitudinal wakefield. Assuming a sinusoidal RF accelerating field,

$$
\Delta E_{\mathrm{RF}}=E_{\mathrm{RF}}(s)\left[\sin \left(z k_{\mathrm{RF}}+\phi_{s}\right)-\sin \left(\phi_{s}\right)\right]
$$

Here, $k_{R F}$ is the wavenumber of the $R F$ and $\phi_{s}$ is the RF phase which is $90^{\circ}$ for maximum acceleration of electrons.

Finally, we will calculate the motion of the bunch centroid. This is important because the centroid, and thus the transport matrices for the central trajectory, depends upon the bunch intensity and the correlated energy spread. ${ }^{[1]}$ The equation for the centroid is found by integrating Eq. (3.2.1) over the particle distribution in $\delta$ and $z$. In general, this is complicated, but if we assume that the transverse trajectory offsets are large compared to the dilutions and we assume that the bunch has a gaussian distribution in $z$, we find

$$
\begin{aligned}
\frac{1}{\gamma(s)} \frac{d}{d s} \gamma(s) \frac{d}{d s} y_{c}(s) & -\left(1-\delta_{\mathrm{av}}\right) \Lambda_{1}\left[y_{c}(s)-y_{q}\right]=\left(1-\delta_{\mathrm{av}}\right) G_{y} \\
& +\left(1-\delta_{\mathrm{av}}\right) \frac{N r_{0}}{\gamma(s)} \frac{W_{\perp 1}^{\prime}(s) \sigma_{z}}{\sqrt{\pi}}\left[y_{c}(s)-y_{a}\right]
\end{aligned}
$$

where $\delta_{a v}$ is the average beam energy and we have approximated the transverse wakefield as $W_{\perp 1}\left(s ; z^{\prime}-z\right)=\left(z^{\prime}-z\right) \|_{\perp 1}^{\prime \prime}(s)$. 
Notice that the dipole wakefield acts like a defocusing term in that it increases the wavelength of a coherent oscillation; of course, the dipole wakefield does not actually have a gradient and thus it does not change the focusing seen by particles within the bunch, i.e., the beta functions are unchanged.

Strictly, we need to include this effect whenever we calculate the bunch trajectory, but, in the NLC main linacs, the wakefield effects are relatively weak. The relative decrease in the phase advance is roughly $\delta \nu / \nu \approx-0.002$ and thus we will neglect the effect. However, in the SLC linac, this effect is much more important.

\subsubsection{Projected Emittance}

In a conservative system, which a linear accelerator approximates, the normalized six-dimensional emittance is conserved. Furthermore, in a conservative system with linear forces, the rms ix-dimensional emittance is conserved. But, in a linear collider, the luminosity is strongly $y^{\prime}$ dependent upon the projection of this emittance into the transverse planes.

As discussed in Appendix A, the six-dimensional rms emittance can be calculated from the beam matrix $\sigma$ which consists of the second moments of the beam distribution:

$$
\epsilon_{6}^{2}=\operatorname{det} \sigma \quad \text { where } \quad \sigma_{i, j}=\left\langle x_{i} x_{j}\right\rangle
$$

the angle brackets denote the average over all beam particles, and $x_{i}$ are the components of the vector: $\left(x_{\beta}, x_{\beta}^{\prime}, y_{\beta}, y_{\beta}^{\prime}, z, \delta\right)$. Similarly, the two-dimensional projected emittances are just calculated from the $2 \times 2$ sub-matrices along the diagonal of the full beam matrix. Specifically,

$$
\epsilon_{y}^{2}=\operatorname{det} \sigma_{y} \quad \text { where } \quad \sigma_{y}=\left(\begin{array}{cc}
\left\langle y^{2}\right\rangle & \left\langle y y^{\prime}\right\rangle \\
\left\langle y y^{\prime}\right\rangle & \left\langle y^{\prime 2}\right\rangle
\end{array}\right) \text {. }
$$

At this point, we should note a very useful method of visualizing the beam. The transverse projected emittance is not conserved because it is coupled to the longitudinal plane. But, since the longitudinal position is fixed, we can divide the beam longitudinally into slices of constant $z$. Each slice has a specified correlated energy deviation which is determined from the bunch compression, the longitudinal waliefieds, and the RF acceleration. Finally, we further subdivide each slice into slices of constant $\delta_{n n}$, the uncurelinted energy deviation. 
Now, as the beam travels down the linkc, the bunch is deformed by the wakefields and the chromatic and dispersive effects. The wakefields depend upon the longitudinal position $z$ while the chromatic and dispersive effects depend upon the energy deviation. Since $z$ and $\delta$ are parameters in the equations of motion, the area of any given slice remains constant, but the centroids of the slices are shifted by dipole wakefields and dispersive errors while the shapes of the slices are changed by chromatic errors and quadrupole wakefields.

So we visualize the beam as being cornposed of all these slices which have different shapes and centroids. The projected enittance is found by summing over all of the slices. Specifically,

$$
\epsilon_{y}^{2}=\operatorname{det}\left[\int d z \int d \delta_{\mathrm{un}} \rho\left(z, \delta_{\mathrm{un}}\right) \sigma_{y}\left(z, \delta_{\mathrm{un}}\right)\right]
$$

where we have neglected the possibility of transverse coupling. Now, using Schwarz's inequality and the positive definite nature of the beam matrix, it is easy to show that any source of correlation with $z$ or $\delta$ increases this projected emittance:

$$
\operatorname{det}\left[\int d z \int d \delta \rho(z, \delta) \sigma(z, \delta)\right] \geq\left(\int d z \int d \delta \rho(z, \delta) \sqrt{\operatorname{det}[\sigma(z, \delta)]}\right)^{2}
$$

where the equality occurs if and only' if the beam matrix does not depend upon either $z$ or $\delta$.

Inequality (3.2.8) is a specific case of a more general result which states that, assuming the beams are initially uncoupled, any source of coupling always increases the smaller of the emittances. In the case of inequality $\mathrm{Eq}$. (3.2.8), the longitudinal emittance has been implicitly assumed to be much much greater than the transverse since the transverse motion does not affect the longitudinal, but a similar result holds for the transverse coupling in the NLC, where the vertical emittance is much smaller than the horizontal; any betatron coupling leads to an increase in the projected vertical emittance. 


\section{CHAPTER 3.3 \\ FILAMENTATION AND TRANSVERSE MATCHING}

In this chapter, we will discuss filamentation and the transverse matching of the beam. The beam extracted from the damping rings has a well defined beam ellipse. This needs to be matched to the machine ellipses of the linacs and the bunch compressors to prevent dilution of the projected transverse emittances. We will first describe the reasons for this requirement and then estimate the effect of mismatches. Most of these effects have been studied by people working on the SLC at SLAC and many of the results are described in Refs. 70, 2, 35, and 73.

\subsubsection{Filamentation and Natural $\beta$ Functions}

Filamentation occurs when particles in the beam have different frequencies of oscillation. The frequency dependence can be due to the energy dependence of the phase advance, i.e., the chromaticity, or nonlinear fields which cause the phase advance to be amplitude dependent. The effect of the filamentation is to cause a phase mixing which makes it difficult to correct dilutions of the projected emittance; once a dilution filaments, it is, for practical purposes, unrecoverable.

Filamentation is important when describing the transient response of the beam, i.e., in linacs or transport lines, or at injection in a storage ring. It is not important when considering the periodic behavior of a beam in a storage ring. Thus, the filamentation is not important when correcting the local (projected) beam size in the damping rings (see Section 2.2) provided that the dilution is corrected in the ring or promptly after the beam is extracted.

The reason for this distinction between the transient and periodic behaviors can be understood by examining the behavior of two harmonic oscillators that have slightly different resonant frequencies; this models the transverse motion of two particles with slightly different energies. If the oscillators are driven off-resonance by the same periodic force, the motion of the oscillators will be similar in both amplitude and phase. In contrast, if both oscillators receive a transient deflection, the amplitude of the resulting oscillations will be similar, but they will have a steadily increasing difference in phase.

We will divide the energy dependent filamentation into two catagories: dispersive and chromatic filamentation, the former applying to filamentation of an oscillation and the later referring to the filamentation of the beam ellipse. Dispersive 


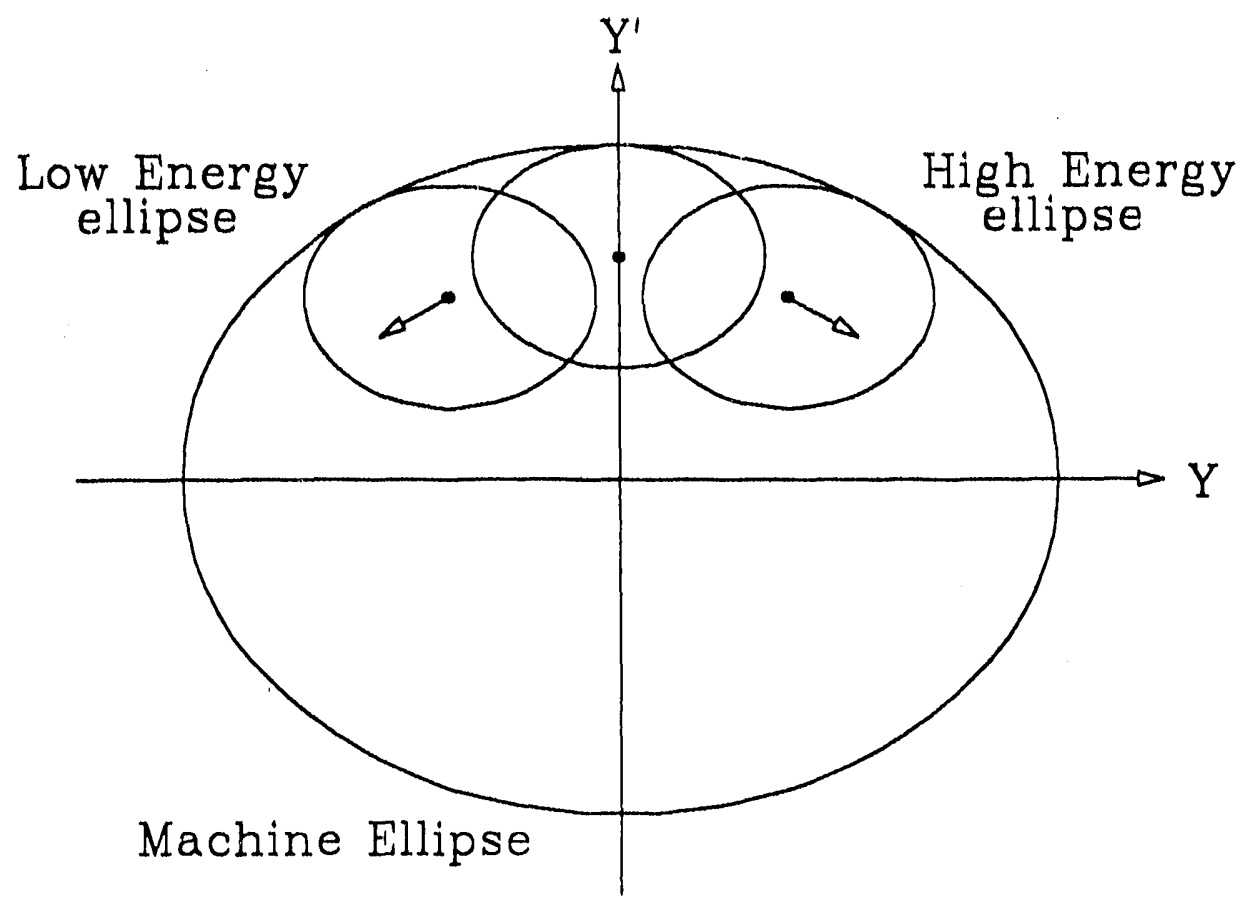

Fig. 19. Dispersive filamentation of a colerent betatron oscillation.

filamentation occurs when the beam performs a coherent oscillation. Here, the centroid of the lower energy particles oscillates at a higher frequency than that of the higher energy particles. The situation in $y-y^{\prime}$ phase space is illustrated schematically in Fig. 19. As the beam filaments, it will fill the annular region in the machine ellipse and the amplitude of the initial colierent oscillation decays.

Chromatic filamentation occur's when the beam is not matched to the focusing structure of the machine; this is illustrated in Fig. 20. Here, the beam ellipse of the low energy particles rotates within the machine ellipse more quickly than that of the higher energy particles. In this case, the beam will filament to fill the machine ellipse at twice the rate of the dispersive filamentation.

In both cases, the projected emittance increases as the beam filaments to occupy the machine ellipse. The machine cllipse is clefined by the beta functions which, as discussed in Appendix A, are a characteristic of the focusing structure, not of the beam. In a storage ring, the beta functions are chosen to be periodic, but, in a transport line, the choice is not as obvious; one needs to determine the boundary conditions. In this section, we will show that there are "natural" beta functions which are determined by the perioclicity of the system. This is well known in storage rings and is intuitively obvious in a long transport line, but it is worth demonstrating explicitly. 
Chapter 3.3.1

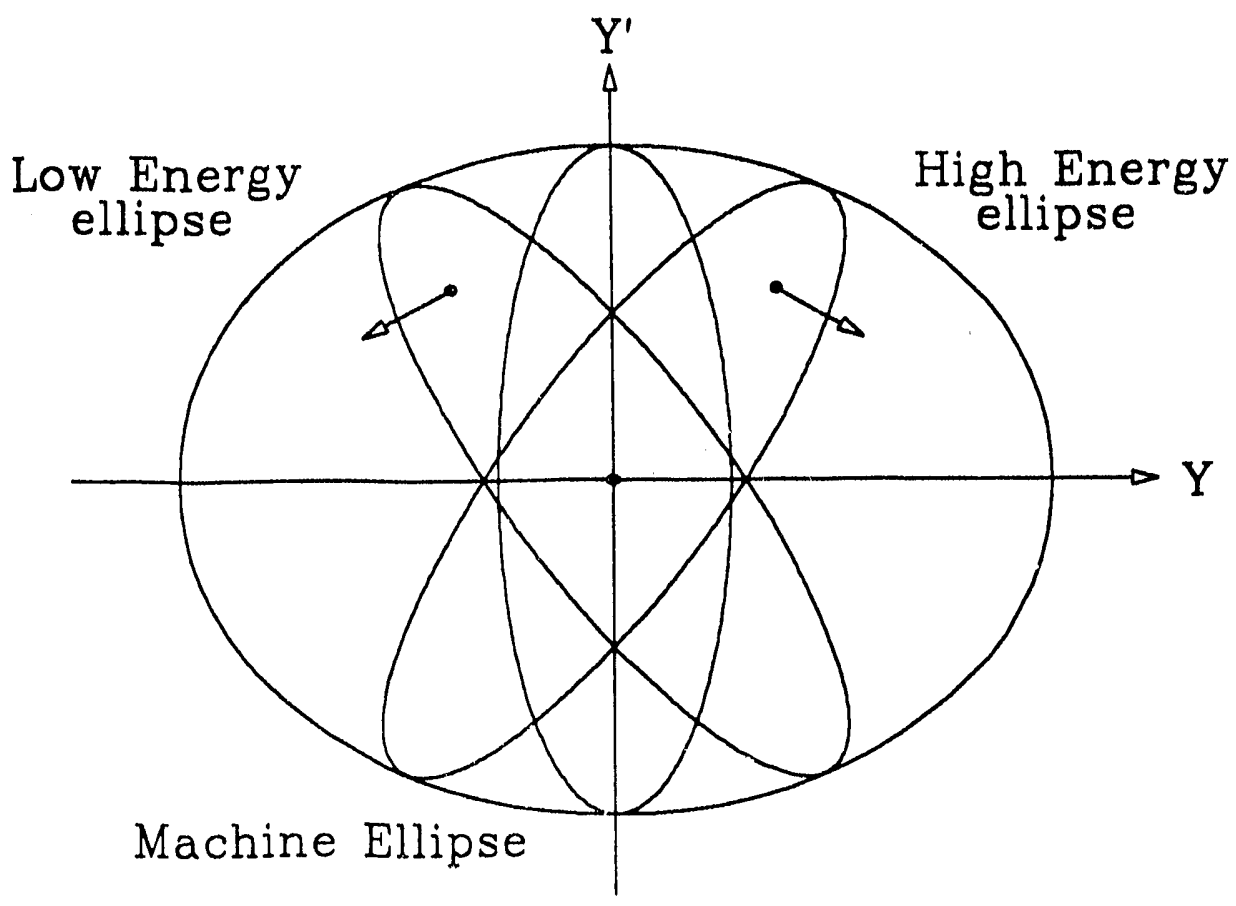

Fig. 20. Chromatic filamentation of a mismatched beam.

To examine the effect of the filamentation, we will consider the transport of the beam matrix $\sigma$ through a periodic lattice. The iwo-dimensional beam matrix equals

$$
\sigma=\left(\begin{array}{cc}
\left\langle x^{2}\right\rangle & \left\langle x x^{\prime}\right\rangle \\
\left\langle x x^{\prime}\right\rangle & \left\langle x^{\prime 2}\right\rangle
\end{array}\right)=\epsilon\left(\begin{array}{cc}
\beta^{\star} & -\alpha^{\star} \\
-\alpha^{\star} & \gamma^{\star}
\end{array}\right)
$$

where we have used the beam parameters introduced in Eq. (A.2.20) in Appendix A.

The transport of a monochromatic beam can be described with the linear transport matrix $\mathrm{R}$.

$$
\boldsymbol{\sigma}(\delta ; s)=\mathbf{R}(\delta ; 0, s) \boldsymbol{\sigma}(\delta ; 0) \widetilde{\mathbf{R}}(\delta ; 0, s)
$$

where $\delta$ is the relative energy deviation of the beam and the transport matrix $\mathbf{R}$ is also introduced in Appendix A. Specifically, the transport matrix for $n$ periodic cells can be written

$$
\mathbf{R}(\delta)=\left(\begin{array}{cc}
\cos n \psi^{\prime} 0(\delta) & \beta_{0}(\delta) \sin n \psi_{0}(\delta) \\
-\frac{1}{\beta_{0}(\delta)} \sin n \psi_{0}(\delta) & \cos n \psi^{\prime}{ }_{0}(\delta)
\end{array}\right)
$$

where the subscript 0 is used to denote the periodic lattice parameters which are functions of the energy deviation and we have assumed that we are starting from 
a symmetry point so $\alpha_{0}=0$. Notice that the determinant of the transport matrix is unity; the transverse rms emittance of a monochromatic beam is not changed by transport through a linear lattice.

Now, we are in a position to demonstrate the existence of the natural lattice functions. Assume that the initial beam is independent of $\delta$ but it has a finite energy spread and an initial emittance $\epsilon$. The inequality Eq. (3.2.8) shows that the projected emittance will increase if $\sigma$ depends upon $\delta$. Assuming that the energy spread is small, we only need consider the first few derivatives of the beam matrix Eq. (3.3.2):

$$
\begin{aligned}
& \frac{d \sigma_{11}}{d \delta}=-\epsilon n \psi_{0}^{\prime}\left(2 \alpha^{\star} \beta_{0} \cos \left(2 n \psi_{0}\right)+\left(\beta^{\star}-\gamma^{\star} \beta_{0}^{2}\right) \sin \left(2 n \psi_{0}\right)\right) \\
& \frac{d \sigma_{12}}{d \delta}=\epsilon \frac{n \psi_{0}^{\prime}}{\beta_{0}}\left(2 \alpha^{\star} \beta_{0} \sin \left(2 n \psi_{0}^{\prime}\right)-\left(\beta^{\star}-\gamma^{\star} \beta_{0}^{2}\right) \cos \left(2 n \psi_{0}\right)\right) \\
& \frac{d^{2} \sigma_{11}}{d \delta^{2}}=\epsilon 2 n^{2} \psi_{0}^{\prime 2}\left(2 \alpha^{\star} \beta_{0} \sin \left(2 n \psi_{0}\right)-\left(\beta^{\star}-\gamma^{\star} \beta_{0}^{2}\right) \cos \left(2 n \psi_{0}^{\prime}\right)\right) \\
& \frac{d^{2} \sigma_{12}}{d \delta^{2}}=\epsilon \frac{2 n^{2} \psi_{0}^{\prime 2}}{\beta_{0}}\left(2 \alpha^{\star} \beta_{0} \cos \left(2 n \psi_{0}\right)+\left(\beta^{\star}-\gamma^{\star} \beta_{0}^{2}\right) \sin \left(2 n \psi_{0}\right)\right),
\end{aligned}
$$

where $\psi^{\prime}=d \psi / d \delta$ and we have assumed that $n \gg 1$; there are also terms that depend upon $d \beta / d s$ and $d \alpha / d s$ but we have only kept terms of highest order in $n$. The derivatives of the other elements of the beam matrix are linear combinations of these.

To prevent dilution of the projected emittance, these derivatives should be zero. One can immediately see that both the first and second derivatives will be zero provided that

$$
\begin{array}{rll}
\alpha^{\star}=0 & \Rightarrow & \alpha^{\star}=\alpha_{0} \\
\beta^{\star}-\gamma^{\star} \beta_{0}^{2}=0 & \Rightarrow & \beta^{\star}=\beta_{0},
\end{array}
$$

i.e., the beam should be matched to the periodic lattice parameters. A mismatched beam will filament, with corresponding emittance growth, until it is matched to these natural lattice functions. This is illustrated in Figs. 21 and 22 which are generated by tracking the beam ellipses of forty macro-particles distributed between $\delta=+3 \sigma_{\epsilon}$ and $\delta=-3 \sigma_{\varepsilon}$. In Fig. 21, we have plotted the projected emittance versus initial beta mismatch for a beam, with a $1 \%$ rms energy spread, that has traversed sixty $90^{\circ}$ FODO cells. The dilution of the projected enitunce has a minimum when the beam is matched to the periodic lattice lunctions. 


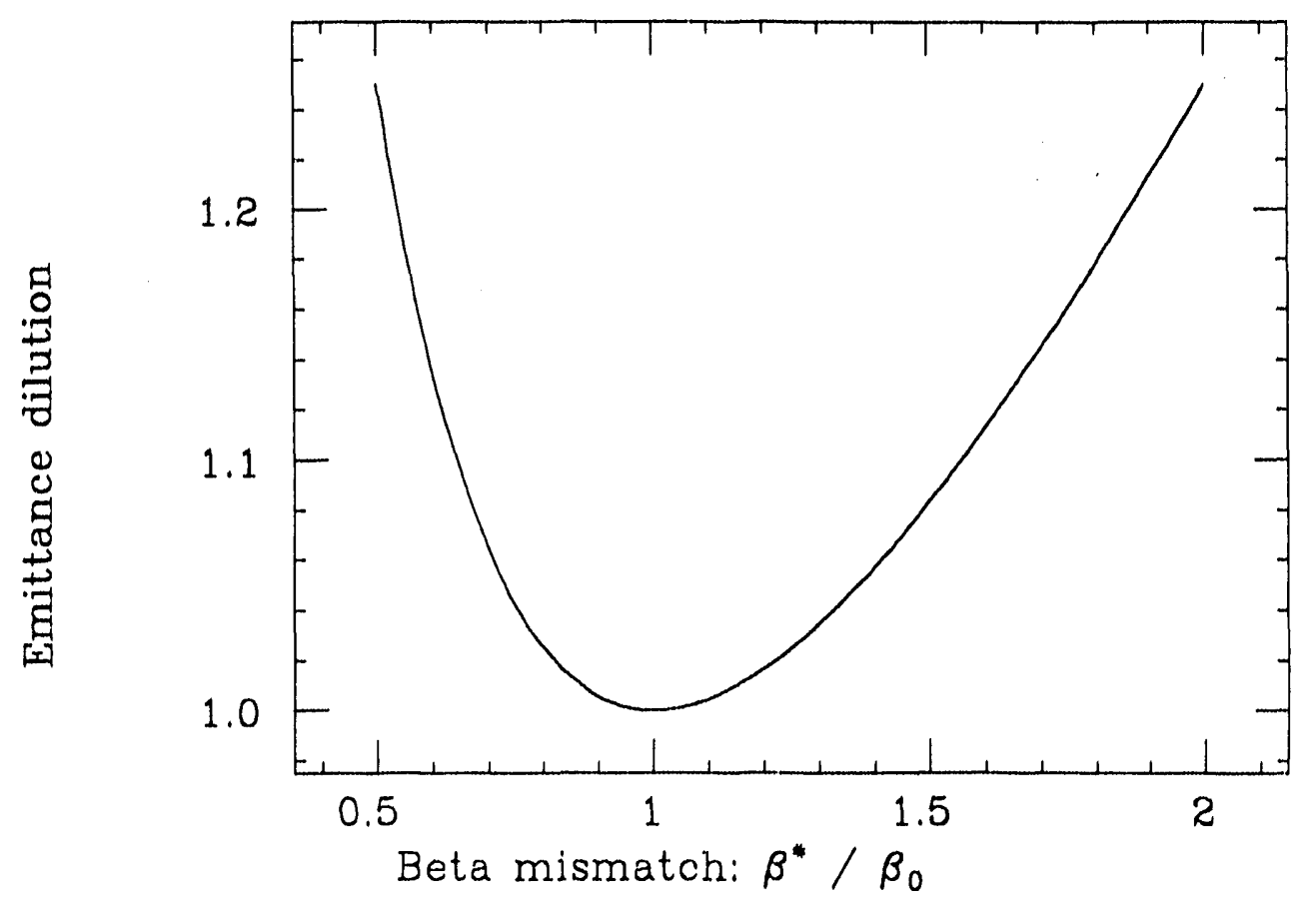

Fig. 21. Simulation of chromatic filamentation in a FODO lattice with $90^{\circ}$ cells and a $1 \%$ rms energy spread vs. the magnitude of the beta mismatch: $\beta^{\star} / \beta_{0}$.

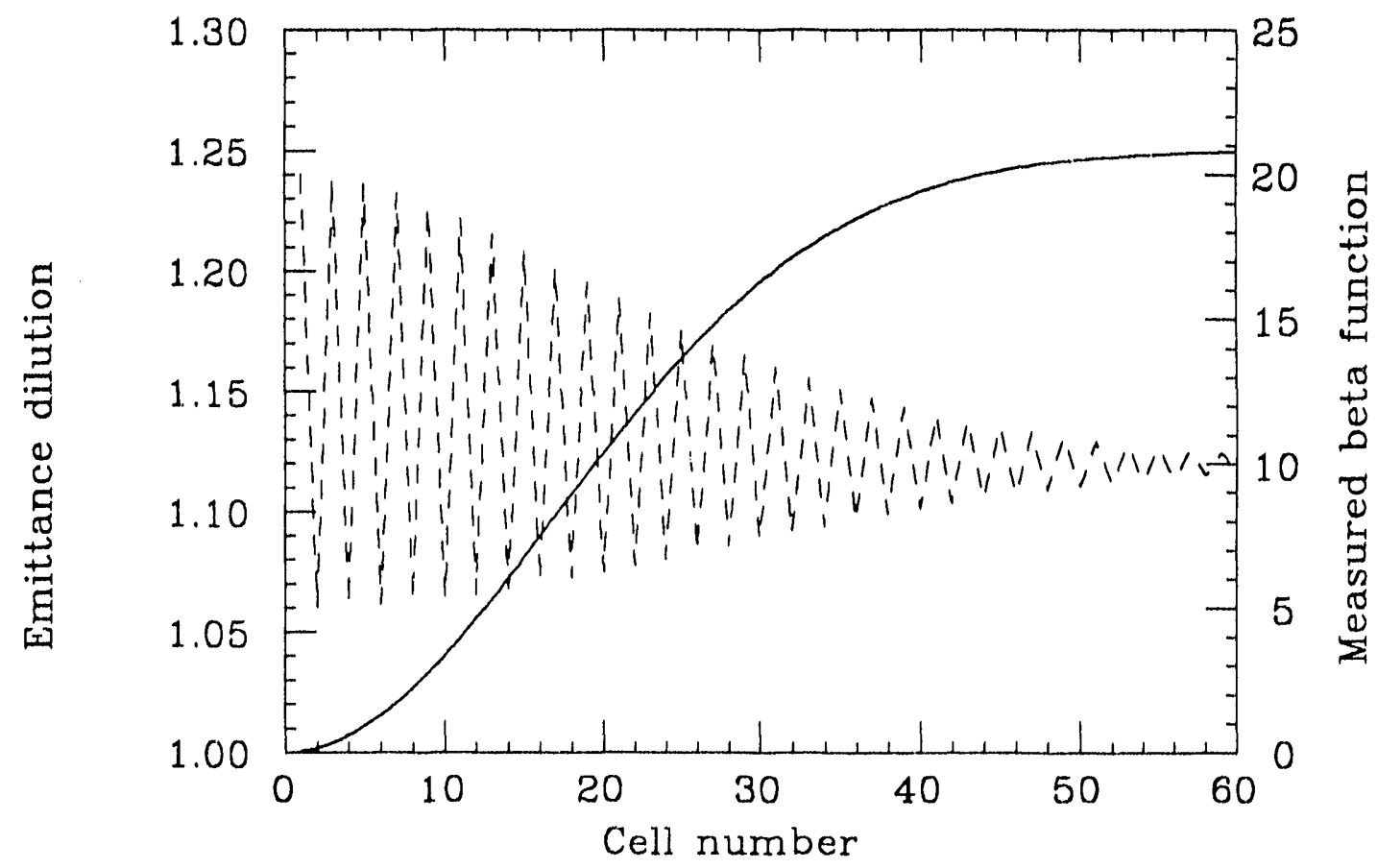

Fig. 22. Simulation of chromatic filamentation in a FODO lattice with $90^{\circ}$ cells and a $1 \%$ rms energy spread; the solid line is the emittance in units of the initial emittance and the dashed line is the measured beta function $\beta^{\star}$. 
In Fig. 22, we have plotted the rms emittance and the measured beta function of the same beam versus cell number. Here, the initial beta mismatch is $\beta^{\star} / \beta_{0}=$ 0.5 . The rms emittance increases towards an asymptotic dilution of $25 \%$ while the measured beta function oscillates about the natural beta function, decaying to the natural value as the beam filaments and the projected emittance grows.

Finally, we need to discuss the degree of filamentation in a linac. This can be estimated from the chromatic phase advance for particles with the rms energy deviation:

$$
\delta \nu=\frac{1}{4 \pi} \int_{0}^{L} \delta K_{1} \beta d s
$$

We will evaluate this using the NLC scaling for a FODO lattice described in Appendix D. Here, the normalized guadrupole strength decreases inversely with the beam energy while the beta functions and the quadrupole and drift lengths increase with the square root of the beam energy: Assuming that the energy spread decreases inversely with the beam energy, we find

$$
\delta \nu=\frac{1}{4 \pi}\left(K_{1} L_{q}\right)_{0}\left(\hat{\beta}_{0}-\ddot{\beta}_{0}\right) \delta_{0} \sum_{n=0}^{N_{\text {cell }}-1} \frac{\gamma_{0}}{\gamma_{n}},
$$

where $\left(K_{1} L_{q}\right)_{0}$ is the initial integrated cuadrupole strength, $\hat{\beta}_{0}$ and $\ddot{\beta}_{0}$ are the initial maximum and minimum beta functions given by Eq. (D.1.1), and $\delta_{0}$ is the initial energy spread. Now, using the relation Eq. (D.1.7) for $\gamma_{n}$, we find

$$
\delta \nu \approx \frac{\delta_{0} \tan \psi_{c}^{\prime} / 2}{\pi} N_{\text {cell }} \sqrt{\frac{\gamma_{0}}{\gamma_{n}}},
$$

where $\psi_{c}$ is the phase advance per cell and we have simplified the expression with Eq. (D.1.3). In the $500 \mathrm{GeV} \mathrm{NLC} \mathrm{linac,} \mathrm{we} \mathrm{find} \delta \nu \approx 0.22$ and thus we conclude that the NLC linac is in the partial filamentation regime; an initial mismatch filaments, but most subsequent dilutions do not. 


\subsubsection{Matching Tolerances}

At this point, we can discuss the required tolerances on matching into the linac. We will consider the effect of beta mismatches, dispersion mismatches, and higherorder contributions, In all cases, we will assume that the mismatch fully filaments. This will over-estimate the severity of the tolerances in the NLC linacs where the beam does not fully filament. Finally, trajectory mismatches, which lead to dispersive filamentation, are discussed in Section 3.4.1.

\section{Lattice Functions}

The emittance dilution due to filamentation of a beta mismatch can be written: ${ }^{(35)}$

$$
\frac{\Delta \epsilon}{\epsilon}=\frac{1}{2}\left(\frac{\beta}{\beta^{\star}}+\frac{\beta^{\star}}{\beta}\right)+\frac{1}{2}\left(\alpha \sqrt{\frac{\beta}{\beta^{\star}}}-\alpha \sqrt{\frac{\beta^{\star}}{\beta}}\right)^{2}-1,
$$

where $\alpha$ and $\beta$ are the machine parameters and $\alpha^{\star}$ and $\beta^{\star}$ are the beam parameters. At a symmetry point where $\alpha^{\star}$ is matched, i.e., $\alpha^{\star}=\alpha=0$, this yields the emittance dilution seen in Fig. 21. Notice that the effect of the beta mismatch is multiplicative. Thus, the tolerances do not decrease as the emittance decreases and the tolerances in the NLC for $6 \%$ emitiance dilution are similar to those in the SLC.

Specifically, to limit the vertical emittance dilution to less than $6 \%$, we need to match the beam parameters and the lattice functions to roughly

$$
\left|\alpha_{y}^{\star}\right| \lesssim 0.20 \quad 0.80 \lesssim\left|\frac{\beta_{y}^{\star}}{\beta_{y}}\right| \lesssim 1.25
$$

where we have assumed that we ale matching to a symmetry point in the lattice. Beta matching at this level is frequently achieved in the SLC despite the non-optimal matching arrangement. ${ }^{(35)}$

Dispersion

Unlike the beta matching, a dispersion mismatch is an additive increase to the emittance. Thus, the tolerance on the vertical dispersion will be much tighter than in the SLC. Assuming full filamentation, the emittance dilution due to residual dispersion at the beginning of the linac is: ${ }^{|73|}$

$$
\Delta \epsilon=\frac{\mathcal{H}_{y} \sigma_{t}^{2}}{2}
$$

where $\mathcal{H}_{y}$ is the dispersion function defined in E( $(A .5 .9)$ and in the NLC linacs, 
this implies a tolerance of

$$
\mathcal{H}_{y} \lesssim \frac{\left(35 \sigma_{y}\right)^{2}}{\beta_{y}} \quad \Rightarrow \quad \hat{\eta_{y}} \lesssim 70 \mu \mathrm{m}
$$

at injection to limit the dilution to $6 \%$.

\section{Nonlinearity}

In the SLC, it has been found that it is extremely important to match the higher-order dispersion and chromaticities at the beginning of the linac. ${ }^{(2)}$ These higher-order terms are supposed to cancel in the bunch compressors, but errors in the lattice reduce the cancellations. Furthermore, these effects were not easy' to correct because the lattice had few independent "knobs" with which one could modify the optics; the problem has been eased with the addition of independent power supplies. We could list tolerances on these higher-order contributions, but, instead, we simply note that the bunch compressors must be designed to facilitate correction of the inevitable error's. 


\section{CHAPTER 3.4 PRIMARY SOURCES OF DILUTION}

In this chapter, we will discuss the primary sources of coupling that increase the projected emittance. These are dispersive errors, transverse wakefields, and RF deflections. In the first two cases, we will consider the effect of a coherent betatron oscillation and a trajectory which is corrected to zero the Beam Position Monitors (BPMs) measurements. In the case of the RF errors, we will only estimate the effect of random errors. Finally, in analogy with Section 2.2.8, we will comment on the distribution of the emittance from these errors and the additional tolerance needed to limit the emittance with a $95 \%$ confidence level. In general, the tolerances found in this section are severe; we will discuss techniques of easing them in Section 3.5.

To estimate these dilutions, we will use two particle models. Here, we represent the beam with two macro-particles separated in $\delta$ or $z$ and then estimate the emittance dilution assuming that the coupling is a linear function of the separation. Instead, one could calculate the dilution as a function of $\delta$ or $z$ and then integrate over the distribution to find the dilution; this approach is taken in Ref. 31. We choose the two particle model since it provides a fairly accurate estimate of the dilution while also providing a simple picture for understanding the correction techniques discussed in Section 3.5.

Finally, throughout this section, we will compare our estimates with the results of computer simulations. These involved tracking the centroids and beam ellipses of 55 macro-particles distributed in the longitudinal phase space in the NLC linac. The simulations included the effect of wakefields and/or dispersive errors as noted in the text.

\subsubsection{Dispersive Errors}

We differentiate between two types of energy dependent errors: dispersive and chrornatic. Dispersive errors arise from a correlation between the centroid of the constant energy slices of the beam and the energy deviation while the chromatic errors are due to a correlation between the second moments of the slices and the energy deviation, i.e., chromatic errors distort the shape of the beam ellipses. The chromatic error was discussed in Section 3.3 as a source of filamentation. If the beam is matched to the lattice, the chromatic error is small. 
The energy deviation includes both an uncorrelated contribution and a contribution correlated with the longitudinal position $z$. The dispersive error due to the correlated energy deviation is used to (partially) cancel the effect of the dipole wakefields; this is BNS damping ${ }^{(8)}$ and will be discussed in the next section. Thus, we categorize this correlated dispersive error with the wakefields and, in these calculations, only consider the dispersive errors due to the uncorrelated energy deviation.

To estimate the effect of the dispersive error, we use a two particle model where one macro-particle has the design energy and one has an energy deviation $\delta_{\mathrm{un}}$. Then, the difference between these two macro-particles $\Delta y_{d}=y_{c}-y_{\delta_{u n}}$ is found from the equation of motion Eq. (3.2.1). This yields

$$
\begin{aligned}
& \frac{1}{\gamma(s)} \frac{d}{d s} \gamma(s) \frac{d}{d s} \Delta y_{d}-\left(1-\delta_{\mathrm{un}}\right) K_{1}(\bar{\delta}) \Delta y_{d}= \\
& \delta_{\mathrm{un}} G_{y}(\bar{\delta})-\delta_{\mathrm{un}} K_{1}(\bar{\delta}) y_{q}+\delta_{\mathrm{un}} K_{1}^{\prime}(\bar{\delta}) y_{c}
\end{aligned}
$$

where $\bar{\delta}$ is the correlated energy spread and $K_{1}(\bar{\delta})=(1-\bar{\delta}) K_{1}$. In addition, we have neglected the dispersive effects of the wakefields, this is valid if the wakefield is weak compared to the external focusing as it is in the NLC.

The solution to this equation can be expressed in terms of the $R_{12}$ transport matrix element. The $R_{12}$ transport element (see Appendix A) describes the position offset resulting from a deflection; it can be thought of as the Greens function for the accelerator lattice. This yields

$$
\begin{aligned}
\Delta y_{d}(s)= & \int_{0}^{s} d s^{\prime} \delta_{u n}\left(s^{\prime}\right)\left(C_{y}^{\prime}-K_{1} y_{q}\right) R_{12}\left(\delta ; s^{\prime}, s\right) \\
& +\int_{0}^{s} d s^{\prime} \delta_{\text {un }}\left(s^{\prime}\right) K_{1} y_{c} R_{12}\left(\delta ; s^{\prime}, s\right)
\end{aligned}
$$

where $\delta=\delta_{\text {un }}+\bar{\delta}$

Note that we have separated the solution into two contributions. If we neglect the slow variation of $\delta_{\text {un }}$, the first contribution is simply proportional to the trajectory at $s$ and can be neglected. The second contribution depends upon the trajectory offsets in all of the chadrupoles along the linac. It is this term that can grow to become a significant emillance clilution. 
At this point, we need to calculate the effect of the dispersion on the projected emittance. If the dispersion is linear in the energy deviation, valid for small $\delta_{u n}$, then the resulting projected emittance can be expressed as ${ }^{\text {(tu) }}$

$$
\epsilon_{y}=\epsilon_{0} \sqrt{1+2 \mathcal{F} / \epsilon_{0}}
$$

where $\epsilon_{0}$ is the undiluted emitance and $\mathcal{F}$ is analogous to the single particle invariant:

$$
2 \mathcal{F}=\gamma_{y} \Delta y^{2}+2 \alpha_{y} \Delta y \Delta y^{\prime}+\beta_{y} \Delta y^{\prime 2}
$$

To limit the luminosity reduction to $3 \%$, we want to limit the emittance dilution to $6 \%$. In this case, the tolerance on $\mathcal{F}$ is $\mathcal{F} \lesssim 0.06 c_{y}$ and tolerance on the expected value of $\Delta y^{2}$ can be expressed as

$$
\frac{\left\langle\Delta y^{2}\right\rangle}{\beta} \lesssim 0.06 \epsilon_{y} \quad \text { or } \quad \Delta y_{\mathrm{rms}} \lesssim 0.25 \sigma_{y}
$$

We will consider the dilution due to two types of trajectories: a coherent betatron oscillation and a trajectory corrected to zero the Beam Position Monitors (BPMs).

\subsubsection{Coherent' Betatron Oscillation}

If the beam performs a betatron oscillation down the linac, it will begin to filament because of the chromatic phase advance. This was referred to as dispersive filamentation in Section 3.3 and is schematically illustrated in Fig. 19 where we have plotted a low-energy, an on-energy, and a high-energy beam ellipse of a beam starting to filament. If the beam fully filaments, the emittance dilution is found from $\mathrm{Eq} .(\mathrm{A} .5 .10)$ to be

$$
\Delta \epsilon_{y}=\frac{1}{2}\left(\gamma_{y} y_{0}^{2}+2 \alpha_{y} y_{0} y_{0}^{\prime}+\beta_{y} y_{0}^{\prime 2}\right)
$$

where $y_{0}$ and $y_{0}^{\prime}$ are the initial amplitudes of the betatron oscillation. For $6 \%$ emittance dilution, this sets a tolerance on the maximum injection jitter of

$$
y_{0} \lesssim 0.35 \sigma_{y}
$$

which corresponds to a tolerance of roughly $0.7 \mu \mathrm{m}$ in the NLC main linacs. 
Equation $(3,4,0)$ specifies the maximum dilution due to beam jitter, but if the beam does not filament significantly, the dilution is smaller and thus the tolerance is looser. To calculate this, we use Eq. (3.4.2) and an expression for a coherent betatron oscillation. Using the $R_{11}$ matrix element, a betatron oscillation can be written:

$$
y_{c}(s)=y_{0} \sqrt{\frac{\beta(s)}{\beta_{0}}} \sqrt{\frac{\gamma_{0}}{\gamma(s)}} \cos \left(\psi(s)-\psi_{0}\right)
$$

where we have assumed the oscillates starts from a symmetry point where $\alpha_{y}=0$.

Now, we use the NLC scaling described in Appendix D. In particular, $K_{1}$ is assumed to decreases inversely with the beam energy while the beta functions and the lengths increase with the square root of the beam energy. This yields a sum over quadrupoles that depends upon the beam energy at the magnets. Using Eq. (D.1.7) and expressing the sum as an integral, we find

$$
\frac{\Delta y_{d}^{2}}{\beta_{f}} \approx \frac{y_{0}^{2}}{\beta_{0}} \delta_{0}^{2} N_{\mathrm{cc} \| l}^{2} 2 \tan ^{2} \frac{\psi_{c}}{2}\left(\frac{\gamma_{0}}{\gamma_{f}}\right)^{2}
$$

where $N_{\text {cell }}$ is the number of FODO colls in the linac, $\delta_{0}$ is the initial uncorrelated energy spread, $\psi_{c}$ is the phase advance per cell, and the expression was simplified with Eq. (D.1.3).

In the $500 \mathrm{GeV}$ NLC linac, Fig. (3.4.9) incorrectly yields a tolerance that is tighter than that of Eq. (3.4.6). This occurs because, as described in Section 3.3, the beam partially filaments in the NLC linacs. The dispersive emittance dilution due to a coherent betatron oscillation in the NLC $500 \mathrm{GeV}$ main linac is plotted in Fig. 23; the dilution due to the walkeficlds is not included. The maximum amplitude of the oscillation is equal to the beam size and thus Eq. (3.4.6) predicts a maximum emittance dilution of $50 \%$. In Fig. 23, the dilution approaches this maximum, reaching $44 \%$ at the end of the linac.

\subsubsection{Conreoted Trajectory}

We will consider the dispersion due to a corrected trajectory essentially following the procedure outlined in Ref. 99. Wo assume that the trajectory is corrected to zero the BPM measurements as is ustinlly the case. Unfortunately, the BPMAs are misaligned, both mechanically and allectronically, and thus, the actual onbit is deflected from side-to-side following the misalignments. 


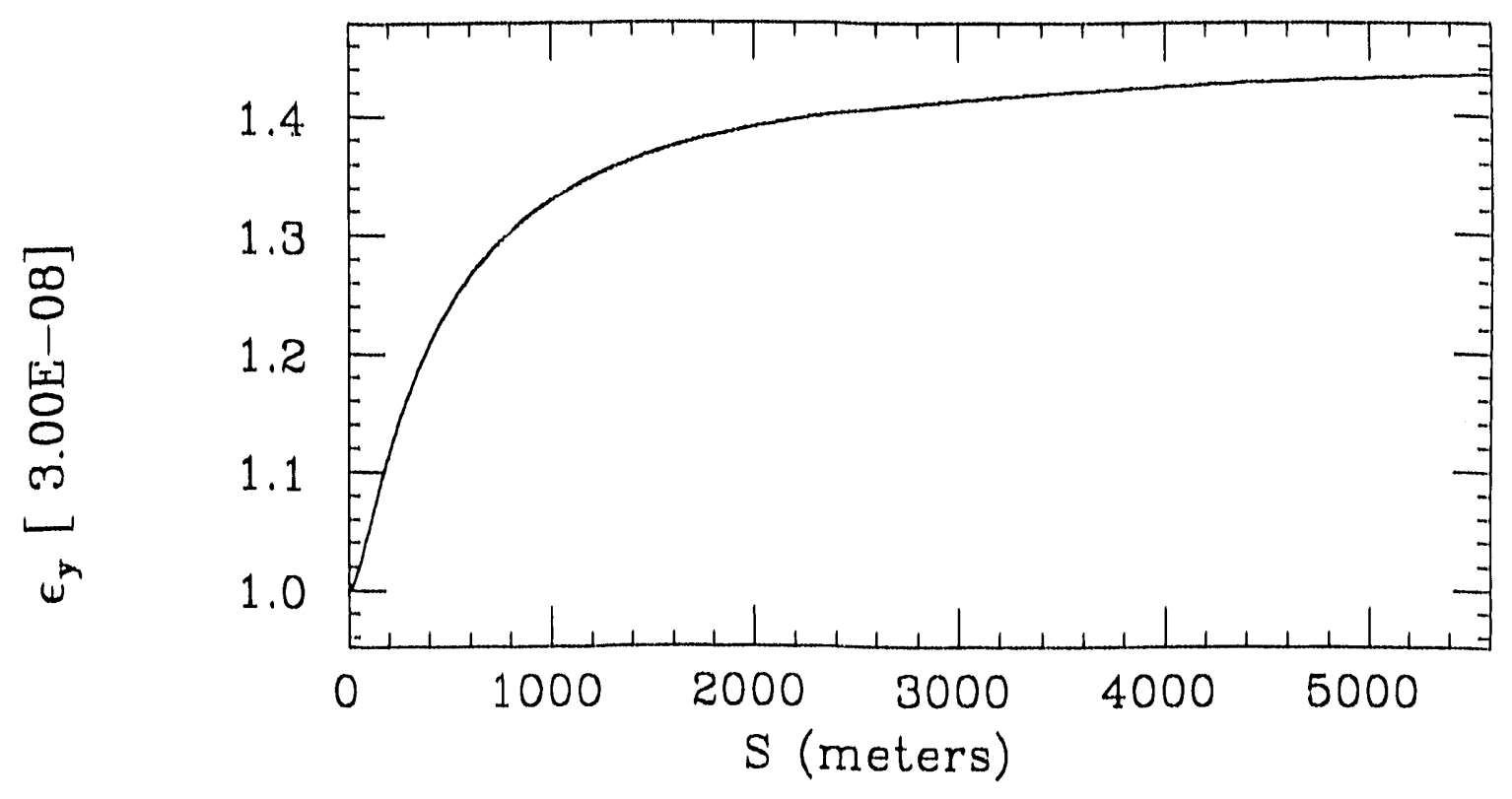

Fig. 23. Simulation of clispersive filamentation of a betatron oscillation in the $500 \mathrm{GeV}$ NLC main linac; the initial oscillation amplitude is equal to the initial beam size.

We will calculate the dispersive error assuming that the BPM misalignments are random and that the trajectory correction only uses correctors and BPMs located at the focusing quadrupoles; we refer to this as "one-to-one" trajectory correction.

To begin, we need to demonstrate that we can neglect the first term of Eq. (3.4.2); then, we can calculate the expected dispersive error. Consider a single misaligned BPM as is illustrated in Fig. 24. Using Eq. (3.4.2), the resulting dispersive error is

$$
\begin{array}{r}
\Delta y_{d}(s)=\left(\delta_{1} \theta_{1} \sqrt{\hat{\beta}_{1}}-\delta_{3} \theta_{3} \sqrt{\hat{\beta}_{3}}\right) \sqrt{\beta(s)} \sin \left(\psi(s)-\psi_{3}\right) \\
+\delta_{2}\left(\kappa_{1} L_{\prime q}\right) \hat{y} \sqrt{\hat{\beta}_{2} \beta(s)} \cos \left(\psi^{\prime}(s)-\psi_{3}\right)
\end{array}
$$

where, for simplicity, we have assumed $90^{\circ}$ phase advance per cell and we have neglected the defocusing quadrupoles. Since the final trajectory is zero, the deflections must cancel: $\theta_{1} \sqrt{\hat{\beta}_{1}}=0_{3} \sqrt{\hat{\beta}_{3}}=\hat{y} / \sqrt{\hat{\beta}_{2}}$. We can neglect the first term if it's magnitude is much less than that of the second, i.e., if

$$
\left(\delta_{1}-\delta_{3}\right) \frac{\hat{y}}{\beta} \ll \delta_{2}\left(K_{1} L_{q}\right) \hat{y}
$$




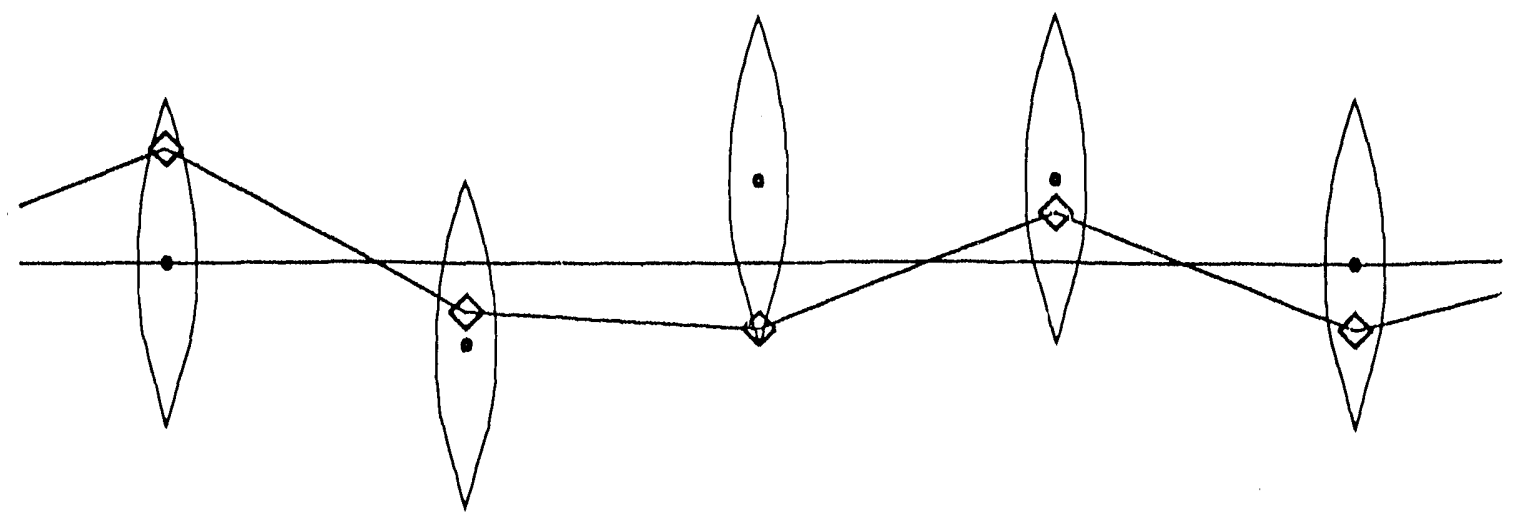

Fig, 24. Schematic of one-to-one trajectory correction; the trajectory is corrected to the BPMs (diamonds) which are misaligned in the quadrupoles which are also misaligned relative to the centerline.

Using the NLC scaling, Eq. (D.1.7), and expressing the integrated quadrupole strength in terms of the beta functions, this condition can be expressed as

$$
\frac{2}{N_{\text {cell }}} \sqrt{\frac{\gamma f}{\gamma_{0}}}=2 \frac{\Delta \gamma}{\gamma} \ll 1
$$

where $\Delta \gamma$ is the energy gain over one cell. This is certainly satisfied in the NLC linacs and thus we will neglect the first term of Eq. $(3.4,2)$.

Now, from the second torm of $E(1,(3.4 .2)$, we find an expected error

$$
\frac{\left\langle\Delta y_{l}^{2}\right\rangle}{\beta} \approx \delta_{0}^{2}\left\langle y_{c}^{2}\right\rangle\left(K_{1} L_{q}\right)_{0}^{2} \frac{\hat{\beta}_{j}}{2}\left(\frac{\gamma 0}{\gamma_{f}}\right)^{3 / 2} \sum_{n=0}^{N_{\text {cell }}-1}\left(\frac{\gamma_{0}}{\gamma_{n}}\right)^{3 / 2}
$$

where we have used the NLC! scaling described in Appendix D. In addition, to simplify the result, we have neglected the defocusing quadrupoles and thus our result will slightly overestimate the erron; He contribution from the defocusing quadrupoles will reduce this resull by a factor of $B / \beta$ which is alosut $15 \%$ in a lattice with a $900^{\circ}$ phase advance per cell. 
Finally, using Eq. (D.1.7) for $\gamma_{n}$ and expressing the sum as an integral, we find

$$
\frac{\left\langle\Delta y_{i}^{2}\right\rangle}{\beta} \approx \delta_{0}^{2}\left\langle y_{c}^{2}\right\rangle \frac{4 \tan ^{2} \psi_{c} / 2}{\hat{\beta}_{f}}\left(\frac{\gamma_{0}}{\gamma_{f}}\right) N_{\text {cell }}
$$

where we have used Eq. (D.1.3) to express the result in terms of the phase advance per cell.

In the $500 \mathrm{GeV} \mathrm{NLC} \mathrm{linac,} \mathrm{this} \mathrm{yields} \mathrm{a} \mathrm{tolerance} \mathrm{on} \mathrm{the} \mathrm{actual} \mathrm{trajectory} \mathrm{of}$

$$
y_{c \mathrm{rms}} \lesssim 4 \mu \mathrm{m}
$$

for $6 \%$ emittance dilution. This is a tolerance on the alignment of the BPMs and it is severe. To verify this result, we have simulated $10 \mu \mathrm{m}$ BPM errors in the NLC 500 $\mathrm{GeV}$ linac. The final dilution, found by averaging the results of 20 different random error distributions, is $\Delta \epsilon_{y}=37 \%$; this agrees well with the prediction Eq. (3.4.14).

Of course, this tolerance assumes that the trajectory follows the random BPM alignment errors. In Section 3.5 we will discuss alternate techniques of correcting the trajectory that could ease this tolerance by over an order of magnitude.

\subsubsection{Transverse Wakefields}

As described in Section 2.3.4, waliefields arise from the electromagnetic interaction between the beam and the external environment. They cause forces that are functions of the longitudinal position with the bunch $z$, and thus, the wakes will dilute the transverse projected emittance. In this section, we will discuss the effect of the wakefields in the linacs. We will only consider a single bunch of particles, neglecting the multi-bunch effects; these are discussed in Ref. 117.

The wakefield force can be separated into multipole components much like the magnetic fields. In a cylindrically symmetric structure ${ }^{[20]}$

$$
F_{y}(z)=N e^{2}\left(\langle y\rangle W_{\perp 1}(z)-2\left\langle x^{2}-y^{2}\right\rangle W_{\perp 2}(z) y+4\langle x y\rangle W_{\perp 2}(z) x+\cdots\right)
$$

where the angle brackets denote an average over the beam particles and $W_{\perp 1}$ and $W_{\perp 2}$ are the dipole and quadrupole wakefields. In general, the magnitude of the higher-order walefield multipoles decleases inversely with the square of the iris radius: $W_{\perp_{n}} \times 1 / a^{2 n}$. Thus, provided that the beam size and the trajectory offsets are small compared to the iris radius, we only need consider the first few multipoles. 
Here, we will first consider the effect of the dipole wakefield due to a coherent betatron oscillation, a corrected trajectory, and random misalignments of the accelerator sections. Then, we will briefly discuss the quadrupole wakefield; it has a small effect. To calculate the effect of the dipole wakefield, we start from the two particle model where one particle is located at the head of the bunch $z=+\sigma_{z}$ and the other particle is at the tail $z=-\sigma_{z}$. Now, half of the difference between these two particles $2 \Delta y_{w}=y\left(z=+\sigma_{z}\right)-y\left(z=-\sigma_{z}\right)$ is found from the equation of motion Eq. (3.2.1). Specifically,

$$
\begin{aligned}
& \frac{1}{\gamma(s)} \frac{d}{d s} \gamma(s) \frac{d}{d s} \Delta y_{w}-(1-2 \bar{\delta}) K_{1} \Delta y_{w}=\bar{\delta}\left(G_{y}-K_{1} y_{q}\right) \\
& +\left(\bar{\delta} K_{1}^{\prime}-\frac{N r_{0}}{4 \gamma} W_{\perp 1}\left(2 \sigma_{z}\right)\right) y_{c}+\frac{N r_{0}}{4 \gamma} W_{\perp 1}\left(2 \sigma_{z}\right) y_{a}
\end{aligned}
$$

where $W_{\perp 1}\left(2 \sigma_{z}\right)$ is the transverse wakefield at $-2 \sigma_{z}$ and $\bar{\delta}$ is half of the coherent energy difference between $z=+\sigma_{z}$ and $z=-\sigma_{z}: 2 \bar{\delta}=\delta\left(z=-\sigma_{z}\right)-\delta\left(z=+\sigma_{z}\right)$.

Again, the solution for $\Delta y_{w}$ can be written in terms of the $R_{12}$ transport matrix elements in a form similar to Eq. (3.4.2). In addition, the resulting dilution can be found from Eq. (3.4.3), where $\mathcal{F}$ is now a function of $\Delta y_{u}$. To limit the luminosity reduction to $3 \%$, we need to limit $\Delta y_{w \prime}$ to $\Delta y_{u \prime} \lesssim 0.25 \sigma_{y}$ or $\Delta y_{w}^{2} / \beta_{y} \lesssim 0.06 \epsilon_{y}$.

\subsubsection{Coheren't Oscillations an'd BNS Damping}

A betatron oscillation can be written in the form of $\mathrm{Eq}$. (3.4.8) and the solution can be found from Eq. (3.4.17) using the $R_{12}$ matrix elements. If the correlated energy spread is zero $\bar{\delta}=0$, the tail particle is driven on resonance by the wakefield of the head particle. Using the NLC scaling and noting that the energy increases linearly with position down a linac, we can express the solution as

$$
\frac{\Delta y_{u 1}^{2}}{\beta_{f}} \approx \frac{y_{0}^{2}}{\beta_{0}}\left(\frac{N^{\prime} \gamma_{0} W_{\perp 1}\left(2 \sigma_{z}\right)}{4}\right)^{2} \frac{\gamma_{0}}{2 \gamma_{f}}\left(\frac{\overline{\beta_{f}}}{\mathcal{G}}\right)^{2}
$$

where $\mathcal{G}$ is the acceleration gradient in $\Delta_{\gamma} p(r)$ meter and $\beta$ is calculated in $A_{p}$ pendix D.

In most cases, this will result in severe tolerances. In the NLC 500 GeV linac, 
this yields a tolerance on the initial amplitude of the coherent oscillation of

$$
y_{0} \lesssim 0.4 \mu \mathrm{m}
$$

for $3 \%$ luminosity reduction and, in the lower gradient $250 \mathrm{GeV}$ linac, this tolerance is a factor of two more severe.

To ease this tolerance, we can add a norrelated energy spread $\bar{\delta}^{[8]}$ This correlated energy spread can have three effects: first, the head and the tail particle have different betatron frequencies because of the chromatic phase advance and thus the resonant growth is reduced; the dilution "beats" rather than growing linearly. Second, the dispersive factor $\bar{\delta} K_{1}^{\prime}$ can be used to directly cancel the wakefield driving term and thereby the growth and, third, the energy spread can cause an apparent damping of an oscillation in the transverse phase space. These techniques are all referred to as BNS damping ${ }^{[6]}$ but we will denote the first two cases as the "beating" and "autophasing" regimes of weak BNS damping and the third as strong BNS damping. Since the NLC is in the weak BNS damping regime, we will only discuss the first two effects.

Weak BNS Damping: Beating

In this regime, the cancellation is a global correction. It depends upon the chromatic phase advance and thus the correction is averaged over many cells. In this case, we can calculate the dilution using a smooth approximation. Explicitly, we assume that the tail macro-particle has a phase advance of $\psi+4 \pi \xi \bar{\delta}$ where $\xi$ is the chromaticity; in a FODO cell the chromaticity per cell is approximately ${ }^{120]}$

$$
\xi_{c}=-\frac{\tan \psi_{c} / 2}{\pi}
$$

Now, if we calculate the effect of a coherent betatron oscillation, neglecting the dispersive contribution, we find

$$
\frac{\Delta y_{w}^{2}}{\beta_{f}} \approx \frac{y_{0}^{2}}{\beta_{0}}\left(\frac{N r_{0} W_{\perp 1}\left(2 \sigma_{z}\right)}{4}\right)^{2} \frac{\gamma_{0}}{2 \gamma_{f}}\left(\frac{\overline{\beta_{f}}}{N_{\text {cell }} \mathcal{G}}\right)^{2}\left(\frac{\sin N_{\text {cell }} 2 \pi \xi_{c} \bar{\delta}}{2 \pi \xi_{c} \bar{\delta}}\right)^{2}
$$

This shows that the dilution beats as the beam propagates down the linac, increasing and decreasing depending upon the relative phase between the head and the tail. 
Ideally, one can minimize the dilution by choosing

$$
\bar{\delta}_{\mathrm{BNS}} \approx \frac{n}{2 N_{\text {cell }} \xi_{c}}
$$

where $n= \pm 1, \pm 2, \cdots$. Of course, when the dispersive effects of the $\bar{\delta} K_{1}$ term are included, one finds that a regative $\bar{\delta}$ is much more effective than a positive $\bar{\delta}$. In the $500 \mathrm{GeV}$ NLC linac, Eq. (3.4.22) yields a minimum energy spread of

$$
\bar{\delta}_{\mathrm{BNS}}=-4 \times 10^{-3}
$$

\section{Weak BNS Damping: Autophasing}

Next, in the autophasing regime, the dispersive term $\bar{\delta} K_{1}$ is used to cancel the wakefield. In the smooth approximation, where $K_{1}$ and $W_{\perp 1}$ are smooth functions of $s$, one can solve for a $\bar{\delta}$ such that the cincellation always occurs. Unfortunately, this local cancellation is not possible in the alternating-gradient focusing structures used in high-energy machines. While the wakefield $W_{\perp 1}$ has a constant sign, an alternating-gradient focusing structure usually contains a periodic array of discrete focusing magnets with both positive and negative $K_{1}$ values. Since the energy spread $\bar{\delta}$ cannot be changed rapidly with $s$, at best one can adjust correlated energy deviation to cancel the integral of the wakefield over a cell of the focusing structure.

To calculate the BNS damping condition, we start with the betatron oscillation of Eq. (3.4.8) and use the $R_{12}$ matrix element to solve Eq. (3.4.17). Since the autophasing cancellation is quasi-local, we can neglect the chromatic phase advance which is only significant after many' cells. 'Thus, we find the expression:

$$
\begin{aligned}
& \frac{\Delta y_{w}}{\sqrt{\beta_{f}}}=\frac{y_{0}}{\sqrt{\beta_{0}}} \sqrt{\frac{\gamma_{0}}{\gamma_{f}}} \sum_{n=0}^{N_{c \in l 1}^{\prime}-1}\left[\frac { \overline { \delta } ( K _ { 1 } L _ { q } ) _ { n } } { 2 } \left((\dot{\beta}-\check{\beta}) \sin \psi_{f}-\right.\right. \\
& \left.\left(\hat{\beta} \cos \psi_{c}-\dot{\bar{\beta}}\right) \sin \left((2 n+1) \psi_{c}-\psi_{f}\right)\right) \\
& \left.-\frac{N r_{0} W_{\perp 1}\left(2 \sigma_{z}\right)}{4 \gamma_{n}}\left(L_{a c c}\right)_{n} \overline{\beta_{n}}\left(\sin \psi_{f}-\sin \left((2 n+1) \psi_{c}-\psi_{f}\right) \cos \psi_{c} / 2\right)\right],
\end{aligned}
$$

where $L_{\text {arc }}$ is the length of arcelerator section between two quadrupoles, i.e. nearly the half of the cell length in sur model. 


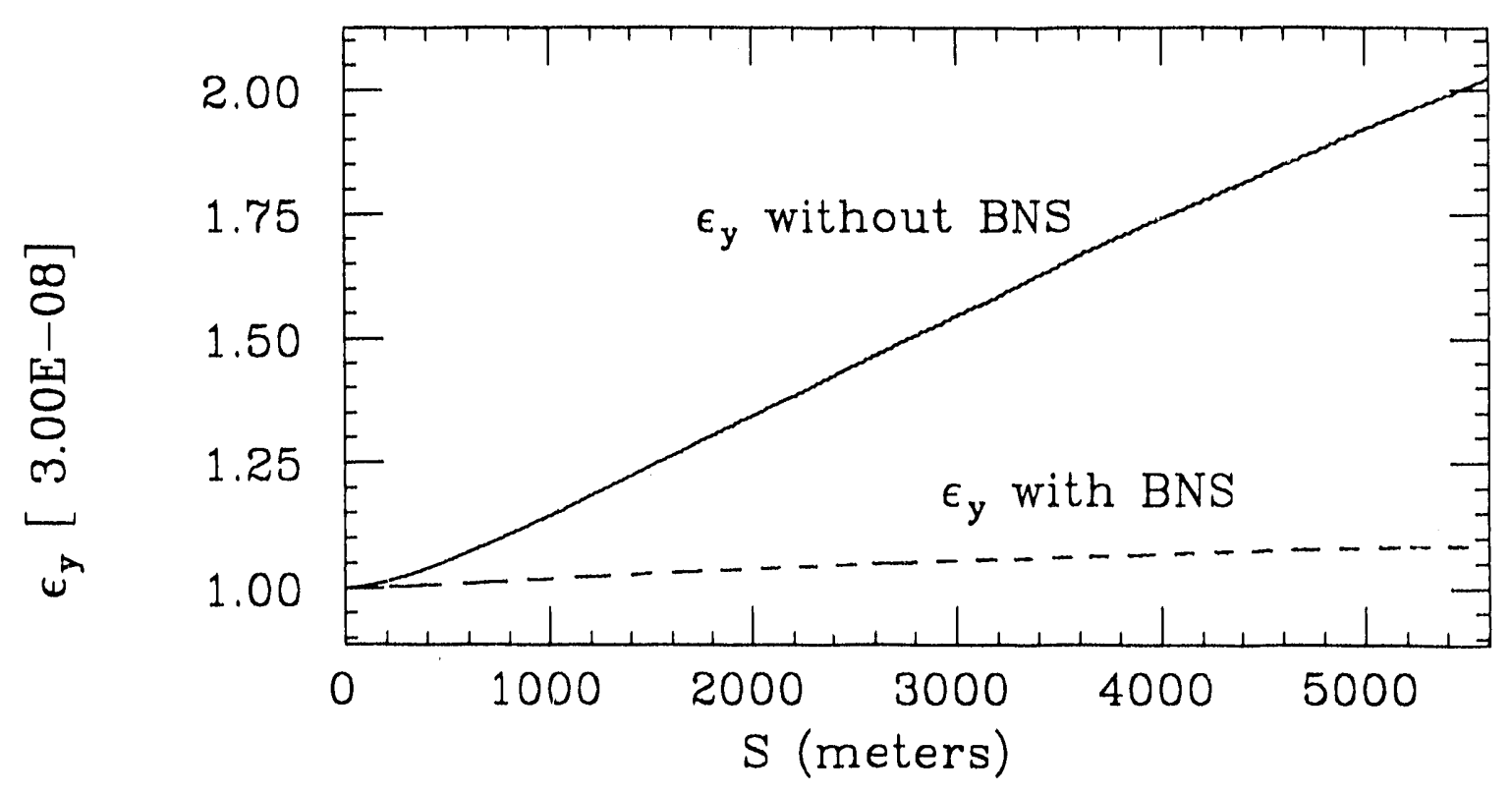

Fig. 25. Simulated emittance dilution due to wakefields with and without BNS damping in the $500 \mathrm{GeV} \mathrm{NLC} \mathrm{linac;} \mathrm{initial} \mathrm{amplitude} \mathrm{of} \mathrm{the} \mathrm{betatron}$ oscillation equals the beam size: $2 \mu \mathrm{m}$.

If we neglect the quickly varying terms, which will average to zero, we find the autophasing condition:

$$
\bar{\delta} K_{1} L_{q}(\hat{\beta}-\check{\beta})=4 \bar{\delta} \tan \psi_{c} / 2 \approx \frac{N r_{0} W_{\perp 1}\left(2 \sigma_{z}\right)}{2 \gamma} L_{a c c} \bar{\beta}
$$

Here, the NLC scaling is convenient since the required $\bar{\delta}$ is independent of $\gamma$. In the $500 \mathrm{GeV}$ NLC linac, we find

$$
\bar{\delta}_{\mathrm{BNS}}=-1.7 \times 10^{-3} .
$$

In the NLC, the correlated energy spread for autophasing is less than that required for the beating BNS damping. In the SLC, the situation is reversed; the energy spread for autophasing is much greater than that for the beating BNS damping.

Of course, in practice it is difficult to achieve the BNS damping conditions for the entire bunch. Thus, even when close to the BNS damping conditions, there is still a small growth of the projected emittance. In Fig. 25 we have plotted the emittance dilution due to wakefields of a beam with and without the optimal autophasing energy spread in the $500 \mathrm{GeV} \mathrm{NLC}$ linac; the initial amplitucle of the betatron 
oscillation is equal to the beam size: $y_{0}=2 \mu \mathrm{m}$. Without BNS damping, the emittance doubles, while with BNS damping, the dilution is only $8 \%$; this implies a tolerance on an oscillation of roughly $2 \mu \mathrm{m}$ with BNS damping and thus the tolerance on the amplitude of the oscillation is determined by the dispersive dilution and not the wakefields.

\subsubsection{Corrected Trajectory}

At this point, we need to consider the effect of the wakefields due to a corrected trajectory where we assume that the trajectory is corrected to zero the BPMs at the focusing quadrupoles. Thus, this dilution will be very similar to the dispersive error due to a corrected trajectory.

As discussed in Section 3.4.1.2, we can neglect the first term: $\left(G_{y}-K_{1} y_{q}\right)$, in Eq. (3.4.17) which gives rise to the non-zero trajectory. This leaves the term proportional to the trajectory. In this estimate, we will assume that the autophasing condition is exactly satisfied. Unfortunately, this does not cancel the wakefields due to a corrected orbit. In particular, the cancellation depends upon the position $x$ in the quadrupoles and the accelerator sections and thus exact cancellation is only possible if $x(s)$ is correlated from point-to-point. This is the case for a coherent betatron oscillation, but it is not true if the particle is steered or deflected by random errors as is the case for a corrected trajectory. Thus, while the BNS technique can cancel the wakefield effects due to a coherent betatron oscillation, it may reduce, but cannot cancel, the effects of waliefields due to a corrected trajectory.

We can estimate the residual dilution using the model illustrated in Fig. 26. We assume that the trajectory is corrected to the (misaligned) BPMs at the focusing quadrupoles and we ignore any misalignments of the defocusing quadrupoles. In this case, the trajectory at the defocusing quadrupoles can be written in terms of the trajectory at the two adjacent focusing quadrupoles:

$$
\begin{aligned}
& y_{-d}=\left(y_{0}+y_{-1}\right) \sqrt{\frac{\hat{\beta}}{\grave{\beta}}} \frac{\sin \psi_{c} / 2}{\sin \psi_{c}} \\
& y_{+d}=\left(y_{0}+y_{+1}\right) \sqrt{\frac{\hat{\beta}}{\check{\beta}}} \frac{\sin \psi_{c} / 2}{\sin \psi_{c}}
\end{aligned}
$$

where we have used the notation of Fig. 26 for the positions at the quadrupoles. Furthermore, the position at the centers of the accelerator sections is just the average of the position in the adjacent focusing and defocusing quadrupoles. 


\section{Vertical trajectory at elements:}

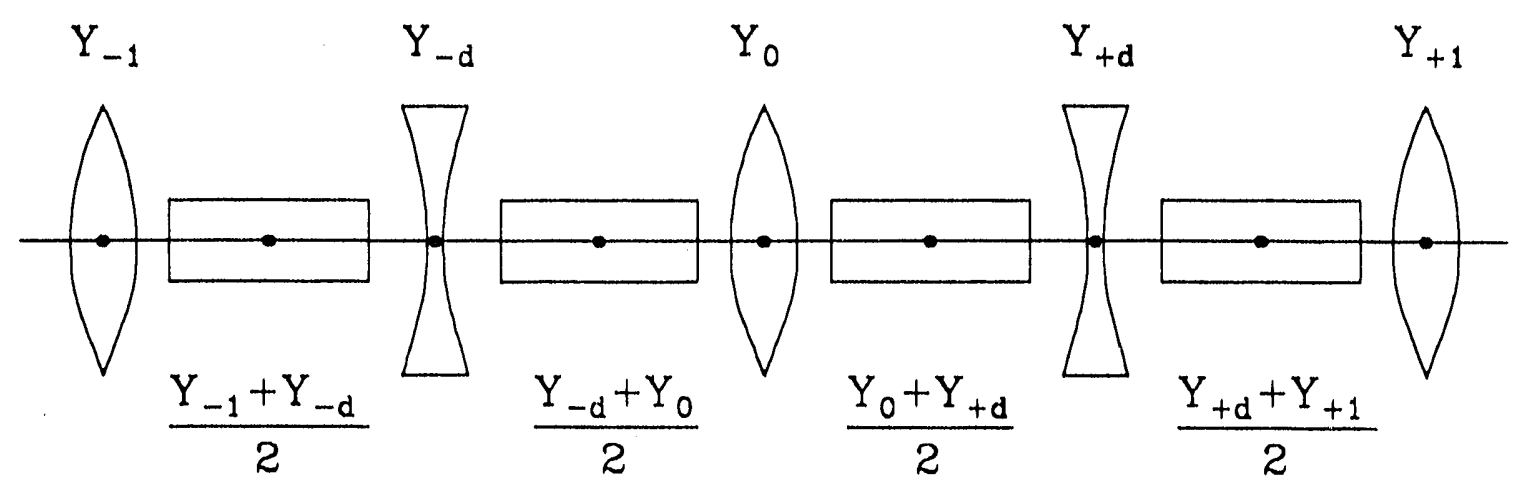

Fig. 26. Schematic of the model used to calculate the wakefield dilution of a corrected orbit; notation denotes the trajectory at the center of the elements.

Now, the dilution can be found using Eq. (3.4.17). Assuming that the trajectory offsets, i.e., the BPM misalignments, are uncorrelated, we find

$$
\begin{aligned}
\frac{\Delta y_{w}^{2}}{\beta_{f}}=\frac{\left\langle y_{\mathrm{BPM}}^{2}\right\rangle}{2} & \sum_{n=0}^{N_{\text {cell }}-1}\left(\bar{\delta}_{\mathrm{BNS}}\left(K_{1} L_{q}\right)_{n}\right)^{2}\left[\sqrt{\hat{\beta}_{n}}\left(1-\frac{\check{\beta}}{\hat{\beta}}\right)\right. \\
& \left.-\frac{\left(\hat{\beta}_{n}-\check{\beta}_{n}\right)}{2 \bar{\beta}_{n}} \sqrt{\overline{\bar{\beta}_{n}}} \cos \bar{\psi}\left(1+\sqrt{\frac{\hat{\beta}_{n}}{\check{\beta}_{n}}}\right)\right]^{2}\left(\frac{\gamma_{n}}{\gamma_{f}}\right),
\end{aligned}
$$

where $\cos \bar{\psi}$ is calculated in Appendix $\mathrm{D}$, we have expressed the wakefield in terms of the BNS damping condition Eq. (3.4.25).

Finally, using the NLC scaling and Eqs. (D.1.7) and (D.1.3), we find

$$
\frac{\Delta y_{u \prime}^{2}}{\beta_{f}} \approx 4\left\langle y_{\mathrm{B} P M}^{2}\right\rangle \frac{\bar{\delta}_{\mathrm{BNS}}^{2} \tan ^{2} \psi_{c} / 2}{\hat{\beta}_{f}}\left[1-\frac{\sin \psi_{c} / 2}{2}\right]^{2} N_{\text {cell }} .
$$

In the $500 \mathrm{GeV}$ NLC main linac, this yjelds a tolerance on the BPM alignment of

$$
y_{\mathrm{BP} M} \lesssim 8 \mu \mathrm{m}
$$

to limit the emittance dilution to $6 \%$. 


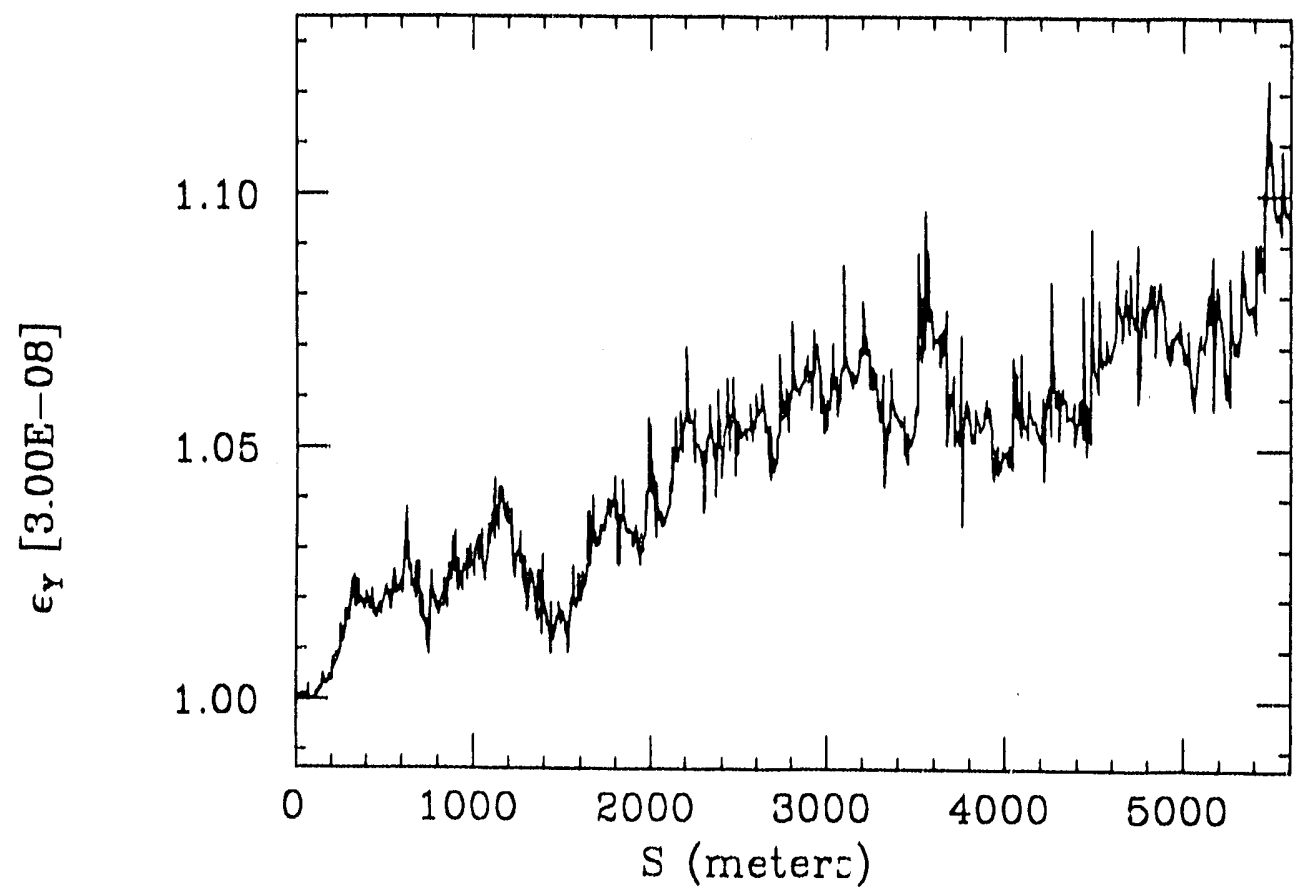

Fig. 27. Simulated watefield dilution in the $500 \mathrm{GeV} \mathrm{NLC} \mathrm{linac} \mathrm{due} \mathrm{to} \mathrm{a}$ corrected trajectory with $10 \mu \mathrm{m}$ BPM random alignment errors; the optimal BNS damping is included.

To verify this result, we have simulated the effect of $10 \mu \mathrm{m}$ BPM alignment errors in the $500 \mathrm{GeV}$ NLC linac; the emittance dilution for a ty'pical case is illustrated in Fig. 27. The final dilution, found by averaging the results of 20 different error distributions, is $\Delta \epsilon_{y}=14 \%$; this implies a tolerance of roughly $6 \mu \mathrm{m}$, slightly tighter that the $8 \mu \mathrm{m}$ calculated above. Again, this is a severe alignment tolerance, but it can be eased with special correction techniques discussed in Section 3.5.

Finally, it is useful to compare the dispersive error due to a corrected trajectory with the wakefield dilution. In general, the dispersive dilution decreases as the focusing decreases while the wakefield dilution increases; $\bar{\delta}_{\mathrm{BNS}}$, in Eq. (3.4.25), decreases with increasing phase advance per cell. This is illustrated in Fig. 28, where we have plotted the dilution arising from a trajectory corrected to BPMs with $10 \mu \mathrm{m} \mathrm{rms}$ alignment errors versus the phase advance per cell in the $500 \mathrm{GeV}$ NLC main linac. The solid line is the total dilution while the dashed and dotted lines are the dispersive and wakefield contributions, respectively; the total dilution is not necessarily the sum of the two individual contributions since cancellations and additive effects occur. Notice that, in the NLC linac, the minimum dilution occurs at roughly $70^{\circ}$ per cell. Although one could conclucle that this lower phase advance per coll would 


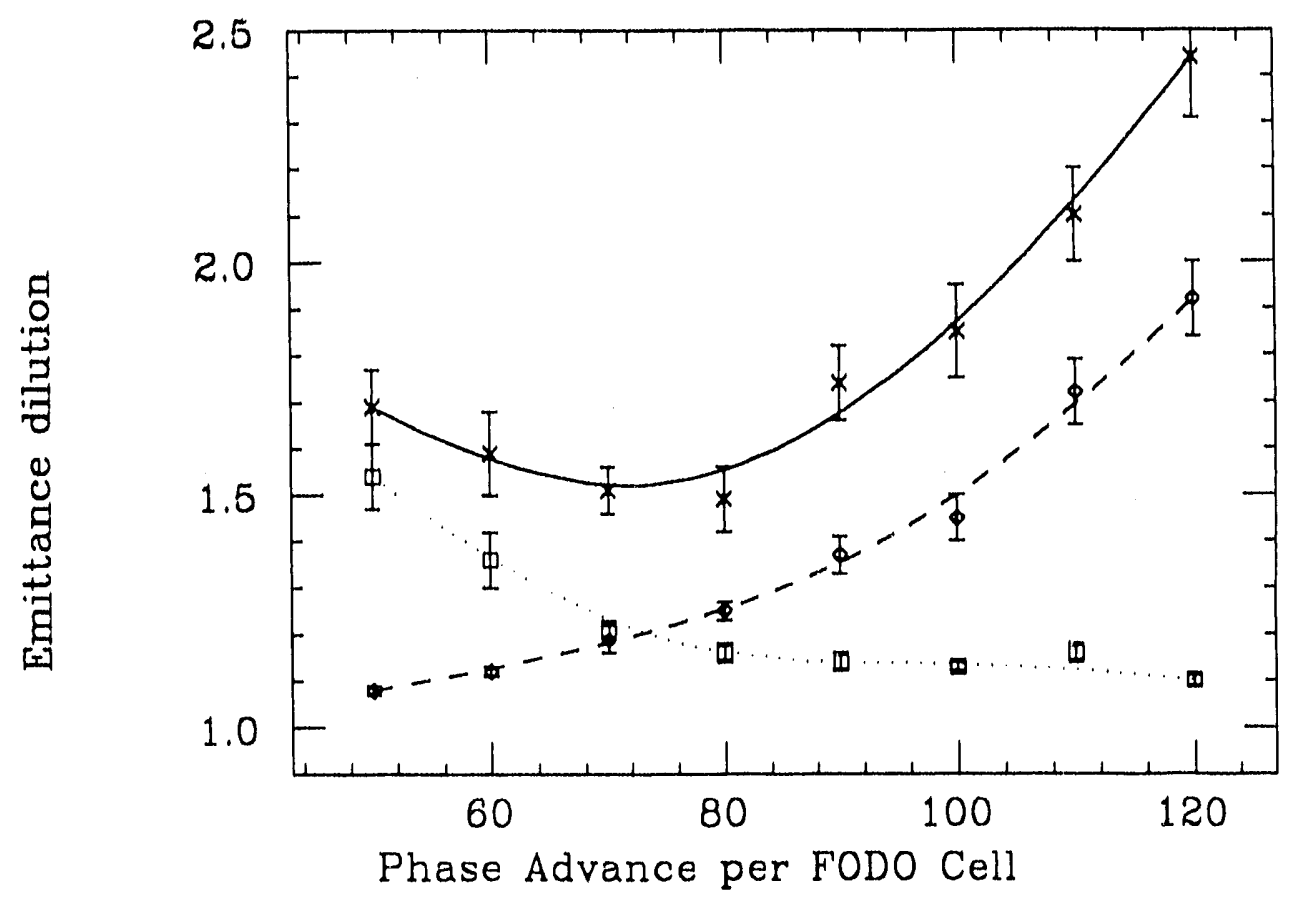

Fig. 28. Simulated emittance dilution in the $500 \mathrm{GeV}$ NLC linac due to a corrected trajectory with $10 \mu \mathrm{m}$ BPM random alignment errors and BNS damping; the solid line is the total dilution while the dashed and dotted lines are the dispersive and wakefield contributions.

be better than the $90^{\circ}$ per cell currently suggested, we need to remember that the effect of the accelerator section misalignments has not been included; these are eased with stronger focusing.

\subsubsection{Accelerator Section Misalignments}

Finally, we can calculate the effect of random accelerator section misalignments. In this case, the BNS damping does not reduce the dilution. Starting from Eq. (3.4.17) and using the NL(? scaling, we find

$$
\frac{\left\langle\Delta y_{w}^{2}\right\rangle}{\beta_{f}} \approx\left\langle y_{a}^{2}\right\rangle\left(\frac{N r_{0} W_{\perp 1}\left(2 \sigma_{z}\right)}{4}\right)^{2} \frac{\overline{\beta_{f}}}{2 N_{\text {cell }}} \frac{1}{\mathcal{G}^{2}}
$$

where $\mathcal{G}$ is the accelerating gradient in $\Delta \gamma /$ meter.

In the $500 \mathrm{GeV} \mathrm{NLC} \mathrm{linac,} \mathrm{this} \mathrm{yields} \mathrm{a} \mathrm{tolerance} \mathrm{on} \mathrm{the} \mathrm{alignment} \mathrm{of} \mathrm{the}$ accelerator sections of

$$
y_{a \mathrm{rms}} \lesssim 4.2 \mu \mathrm{m} \quad,
$$

to limit the emittance dilution to fi\%. We will discuss techniques of easing this 
tolerance in Section 3.6.

\subsubsection{QUADRUPOLE WAKEFIELD}

As expressed in Eq. (3.4.16), the quadrupole wakefield is proportional to the second moments $\left\langle x^{2}-y^{2}\right\rangle$ and $\langle x y\rangle$ of the beam. It generates a quadrupole or skew quadrupole force that varies along the length of the beam. Thus, the wakefield causes a $z$ dependent betatron mismatch or a $z$ dependent betatron coupling; both effects increase the vertical emittance of a flat beam. Furthermore, in an alternating gradient lattice, the second moments of the beam are usually non-zero, even if the trajectory is corrected perfectly. Thus, the wakefield causes a "fundamental" dilution of the vertical emittance of an intense beam; these effects were first analyzed in Ref. 30.

In the NLC linacs, this fundamental clilution is insignificant because the beam size is tiny compared to the iris radius of the accelerating structures. Instead, the quadrupole wakefield may prove important because it can couple large horizontal trajectory offsets into the vertical plane. We can estimate the effect of the quadrupole wakefield using the two particle model for $\Delta y_{w}$ described earlier. Using the force in $\mathrm{Eq} .(3.4 .16)$, we find a solution for $\Delta y_{w}$ similar to $\mathrm{Eq} .(3.4 .2)$ :

$$
\Delta y_{w}=-\int_{0}^{s} d s^{\prime} \frac{N r_{0}}{\gamma} W_{\perp 2}\left(2 \sigma_{z}\right) x_{c}^{2} y_{c} R_{12}\left(2 \bar{\delta}_{;} s^{\prime}, s\right)
$$

where $x_{c}$ is the horizontal beam trajectory'

We consider the effect of coherent betatron oscillations in both planes; the oscillations are given by $\mathrm{Eq}$. (3.4.8). In this case, we use Eq. (3.4.33) to find

$$
\frac{\Delta y_{w}^{2}}{\beta_{f}} \approx \frac{1}{2}\left(\frac{N r_{0} W_{\perp 2}}{4 \mathcal{G}}\right)^{2} \frac{x_{0}^{4} y_{0}^{2}}{\beta_{x 0}^{2} \beta_{y 0}}{\overline{\beta_{x 0}}}^{2}{\overline{\beta_{y 0}}}^{2} \frac{\gamma_{0}}{\gamma_{f}} \ln ^{2} \frac{\gamma_{f}}{\gamma_{0}}
$$

where $x_{0}$ and $y_{0}$ are the initial amplitudes of the betatron oscillations and $\mathcal{G}$ is the acceleration gradient. Here, we have assumed that the horizontal and vertical phase advance per cell are separated; the result should be doubled for the case of equal phase advances per cell.

To use this equation, we need the quadrupole wakefield at $2 \sigma_{z}$. We will estimate this by simply scaling the dipole wakefield. In general, the quadrupole wakefield is 
a factor of $(1 / a)^{2}$ smaller that the dipole wakefield; ${ }^{|20|} a$ is the accelerator structure iris radius which is $a \approx 4 \mathrm{~mm}$ in the NLC main linacs $(11.4 \mathrm{GHz})$ and $a \approx 1.5 \mathrm{~cm}$ in the preliminary linacs $(2.8 \mathrm{GHz})$.

Thus, in the $500 \mathrm{GeV}$ NLC linac, we find $W_{\perp 2}\left(2 \sigma_{z}\right) \sim 10^{11} \mathrm{~m}^{-5}$ for a bunch length of $100 \mu \mathrm{m}$. Now, $\mathrm{Ec}$. (3.4.34) predicts an emittance dilution of less than $10^{-4}$ assuming a $100 \mu \mathrm{m}$ horizontal betation oscillation and a $10 \mu \mathrm{m}$ vertical oscillation. Thus, this is an insigniflcant effect even though we have assumed extremely large amplitudes for the initial betatron oscillations.

\subsubsection{RF Deflections}

RF deflections occur if the accelerating field is not oriented in the direction of the beam propagation. This can be due to misalignments of the RF structures, errors with the RF structures such as tilted irises, or an angular trajectory through the structure.

Assuming a sinusoidal RF field, the deflection can be written ${ }^{(108)}$

$$
\Delta y^{\prime}=g \frac{\Delta \gamma_{\mathrm{RF}}}{\gamma} \sin \left(z k_{\mathrm{RF}}+\phi_{0}\right)
$$

where $\Delta \gamma_{\mathrm{RF}}$ is the acceleration from the structure, $k_{\mathrm{RF}}$ is the RF wavenumber, and $\phi_{0}$ is the phase of the deflection relative to the bunch; this is not necessarily equal to the phase of the accelerating field. Finally, $g$ is the longitudinal-transverse coupling. This can be due to misaligned section, a non-zero trajectory through the section, input/output coupler asymetries, and construction error's in the sections. In these case, $g$ is:

$$
g= \begin{cases}\Theta_{\mathrm{acc}} / 2 & \text { for misaligned sections } \\
y^{\prime} / 2 & \text { for trajectory error } \\
\lesssim \Theta_{\mathrm{iris}} & \text { for systematic tilted irises }\end{cases}
$$

The factor of $\frac{1}{2}$ appearing in the first two expressions arises from the radial focusing at the ends of the structure while the effect of tilted irises is much more complex since the cavity fields are perturbed; using simple analysis, one estimates $g$ to be between $\Theta_{\text {iris }}$ and $\Theta_{\text {iris }} / 2$.

The deflections have two effects: they deflect the beam centroid and they cause a deflection that is correlated with the longitudinal position within the bunch $z$. The 
former effect is simply corrected with dipole correctors, but the later effect dilutes the projected emittance in a manner similar to the dilution due to the transverse wakefields.

We can estimate the dilution in the same manner that we calculated the effect of the wakefelds. Assuming uncorrelated errors with random phases $\phi_{0}$ and using the NLC scaling, we find

$$
\frac{\left\langle\Delta y_{\mathrm{RF}}^{2}\right\rangle}{\beta_{f}}=\left(\sigma_{z} k_{R \mathrm{~F}}\right)^{2}\left\langle g^{2}\right\rangle \frac{\overline{\beta_{f}}}{4}\left(\frac{\Delta \gamma_{\mathrm{llF}}}{\gamma_{f}}\right)^{2} \sum_{n=0}^{N_{\mathrm{aca}}-1}\left(\frac{\gamma_{f}}{\gamma_{n}}\right)^{1 / 2}
$$

where $2 \Delta y_{\mathrm{RF}}=y\left(z=+\sigma_{z}\right) \cdot y\left(z=-\sigma_{z}\right)$ and $N_{a c c}$ is the number of accelerator sections. Finally, we express the sum as an integral and note that $\gamma$ increases linearly with the number of accelerator sections, finding

$$
\frac{\left\langle\Delta y_{\mathrm{RF}}^{2}\right\rangle}{\beta_{f}} \approx\left(\sigma_{z} \operatorname{liRF}^{2}\right)^{2}\left\langle g^{2}\right\rangle \frac{\overline{\beta_{f}}}{2}\left(\frac{\Delta_{\gamma_{\mathrm{RF}}}}{\gamma_{f}}\right) .
$$

In the $500 \mathrm{Gev} N L C$ linac, $\mathrm{Eq} \cdot\left(3.4 .38^{\circ}\right)$ yields a tolerance of

$$
g_{r m s} \lesssim 31 \mu 1^{\prime} a d
$$

for $6 \%$ emittance clilution. This is not a severe tolerance on the trajectory or the alignment; at the beginning of the linac, it corresponds to a $120 \mu \mathrm{m}$ orbit tolerance in the focusing quadrupoles and a $80 \mu \mathrm{m}$ tolerance on aligning the ends of each structure. But, it is a severe construction tolerance on the systematic alignment of the irises in the accelerator sections. Furthermore, despite the lower gradient, the tolerance is comparable in the preliminary linac since it is much lower energy. Specifically, in the weak focusing preliminary linac, we find

$$
g_{\mathrm{r} m \mathrm{~m}} \lesssim 50 \mu \mathrm{rad}
$$

for $6 \%$ emittance dilution; the stronger focusing version has a looser tolerance. For comparison, the measured value in the SLC accelerator sections is $g_{\text {rms }} \sim$ $200 \mu \mathrm{rad}^{[107)}$

Finally, we note two points: first, these effects can also be very severe in the bunch compressors since the bunch is long in what is usually a very high gradient structure. ${ }^{[08]}$ Second, since the effoct of these RF kicks is similar to that of the transverse dipole wakefield, we can correct the effect of the RF deflections with the wakefields. This will be discussed further in Section 3.6 . 


\subsubsection{Distributions}

Throughout this section, we have calculated the eapected dilution from random errors. Of course, the dilution has a distribution and when specifying a tolerance, we need to include the confidence that, given the tolerance, the dilution will be less than the desired limit. This issue has been discussed in Section 2.2 .8 with respect to emittance dilutions in storage rings.

In a linac, we can quickly conclude that the dilution function $\mathcal{F}$ will have an exponential distribution if the alignment errors have gaussian distributions. Thus, small emittance dilutions should also have exponential distributions; using Eq. (3.4.3), the emittance dilution can be written

$$
\frac{\Delta \epsilon}{\epsilon_{0}} \approx \frac{\mathcal{F}}{\epsilon_{0}}
$$

if the dilution is small.

Unfortunately, as noted in Section 2.2.8, an exponential distribution has a $95 \%$ confidence level at three times the expected value. Thus, if one wants to specify tolerances that will limit the emillance dilution for $95 \%$ of the possible arrangements of errors, the tolerances given in this section need to be divided by a factor of $\sqrt{3}$. Fortunately, unlike a storage ring, in a linac we are specifying many unrelated tolerances that all limit the respective dilutions to $6 \%$. Thus, we do not need to specify each individual tolerance with a $95 \%$ CL to limit the sum of all of the independent effects with a $95 \%$ confidence level. The final distribution is found by convolving all of the individual exponential distributions and will have the form of a $\chi$-squared distribution with $2 N$ degrees of freedom, where $N$ is the number of individual tolerances. 'This distribution becomes narrowly' centered about the expected value as $N$ increases and thus the value of $f_{\mathrm{CL}}$ needed to scale from the expected value to the $95 \%$ conficlence value will be much less than three. 


\section{CHAPTER $\quad 3.5$ \\ TRAJECTORY CORRECTION}

\subsubsection{Introduction}

As discussed in Sections 3.4.1.2 and 3.4.2.2, standard trajectory correction techniques steer tha beam to zero the BPM measurements; we generically refer to these techniques as "one-to-one" correction since typically a single upstream corrector is used to zero a single downstream BPM. The problem with this approach is that the BPMs are typically misaligned, both electronically and mechanically. Thus, the corrected trajectory is kicked from side to side, following the BPM misalignments. In such a case, we found that the dilutions due to the dispersive errors and the wakefields tend to grow with the square root of the number of BPMs. In the $500 \mathrm{GeV}$ NLC linac, we found tolerances on the BPM alignment of roughly $\sigma_{\mathrm{BPM}} \lesssim 4 \mu \mathrm{m}$ for $6 \%$ emittance dilution. This is over one order of magnitude better alignment than can be achieved with techniques now in practice.

To avoid this situation, we need to either find and correct the BPM misalignments or develop an algorithm which does not depend upon the BPM alignment errors. A method of using the beam to do the former has been developed for the SLC linac. ${ }^{[3,07]}$ This "beam-based" alignment technique uses trajectory information from two or more different focusing configurations to solve for the individual misalignments of the quadrupoles and the BPMs; the focusing configuration of the machine is varied by tuning the quadrupole magnets which focus the beam throughout the linac. This method has been used very successfully to find most of the large alignment errors in the SLC linac with an accuracy of roughly $100 \mu \mathrm{m}^{(3)}$

In this section, we discuss the other approach, namely, the use of correction algorithms that are less dependent on the BPM alignment errors. Specifically, we will discuss two methods referred to as "Dispersion-Free" (DF) correction ${ }^{[85,86]}$ and "Wake-Free" (WF) correction; ${ }^{[87,88 \mid}$ the DF technique reduces the dispersive dilution due to a corrected trajectory while the WF method reduces both the dispersive and the wakefield dilution arising from a corrected beam trajectory.

Our basic approach is similar to that of the beam-based alignment of Refs. 3 and 67 in that we use information from two or more different focusing configurations. Specifically, we measure trajectories where the focusing structure is changed between measurements. By subtracting these trajectories, the resulting difference orbits are 
independent of the BPM alignment errors. In theory, the quadrupole misalignments could now be found. Unfortunately' the difference orbits still have errors due to beam jitter, finite BPM precision, and additional unknown deflections. Rather than trying to solve for the individual misalignments, we simply correct the trajectory to minimize these difference orbits; this will then minimize the dispersive error and/or the wakefleld dilution.

In the next sections, we will first describe the DF correction algorithm. We present the basic algorithm and then perform a detailed error analysis to determine the limitations of the technique. Finally, we verify the performance of the technique with simulations on the NLC linac. Next, we introduce the WF technique. This is a simple extension of the DF algorithm. Again, we present the results of simulations illustrating the effectiveness of the correction algorithm. Finally, we describe some extensions to these techniques and we discuss the relative merits of these trajectory correction algorithms and the beam-based alignment technique. Much of the material in these sections is taken directly from Refs. 85 and 87.

\subsubsection{DF Correction}

The DF correction technique is quite intuitive. To correct the dispersive emittance dilution, we simply correct the energy dependence of the beam trajectory. This can done by varying the effective beam energy and then correcting the difference between the resulting orbit and the original trajectory; in a linac, the effective beam energy can be varied by either changing the actual beam energy or, equivalently, changing the magnet strengths. Physically, it is easiest to understand this algorithm in terms of the dispersive error expressed in Eqs. (3.4.1) and (3.4.2) of Section 3.4.1. In essence, the DF algorithm finds a trajectory $y_{c}(s)$ such that over any short region of the accelerator the integrals in expression Eq. (3.4.2) are small.

We should note that the technique is very similar to the dispersion correction used in storage rings. Actually, the process is simpler in a linac than in a storage ring since one can make larger effective energy changes, increasing the measurement precision, and there are no nonlinear sextupole magnets which can confuse the measurement.

To correct the difference orbit resulting from changing the effective beam energy, we need to measure the beam trajectories. If we only consider transverse deflections 
due to quadrupole misalignments and dipole correctors, the ith BPM will measure

$$
m_{i}=\lambda_{i}^{\prime}\left(t_{1}\right)-b_{i}+x_{0} R_{11}\left(s_{0}, s_{i}\right)+x_{0}^{\prime} R_{12}\left(s_{0}, s_{i}\right)+\sum_{j} \theta_{j} R_{12}\left(s_{j}, s_{i}\right)
$$

where $\chi$ is a stochastic variable representing the BPM precision error, i.e, the reading-to-reading jitter of the BPM measurement, $\theta$ is the integrated deflection of the dipole correctors and the quadrupole misalignments, and $b$ is the BPM misalignment relative to the linac centerline. Finally, the matrix coefficients $R_{11}$ and $R_{12}$ describe the motion of the beam centroid and thus they implicitly include the effect of the wakefields and filamentation.

In a similar manner, we can calculate the measured trajectory after the effective beam energy is changed by $\delta(s)$. The difference orbit that we will correct is found by subtracting this new trajectory from the original measured trajectory:

$$
\begin{aligned}
\Delta m_{i}=\left(\chi_{i}\left(t_{1}\right)-\chi_{i}\left(t_{2}\right)\right) & +x_{0} \overline{R_{11}}\left(\delta_{i} s_{0}, s_{i}\right)+x_{0}^{\prime} \overline{R_{12}}\left(\delta ; s_{0}, s_{i}\right) \\
& +\sum_{j=1} \theta_{j}\left(R_{12}\left(s_{j}, s_{i}\right)-\frac{R_{12}\left(\delta ; s_{j}, s_{i}\right)}{1+\delta}\right) .
\end{aligned}
$$

Here, $\bar{R}(\delta) \equiv R-R(\delta)$ and $R(\delta)$ is the coefficient $R$ calculated for the modified optics due to the change in energy. It is important to notice that the difference orbit is independent of the BPM misalignments. We should also note that we have not included additional unknown errors such as RF deflections or magnetic strength errors; these and other errors will be discussed in Section 3.5.3.

In principle, using $\left(N_{q}+2\right)$ BPMs, we could solve for the $N_{q}$ quadrupole misalignments and the initial conditions exactly', provided that the BPM precision errors and any unknown deflections are negligible. In such a case, we could fix the injection and the quadrupole errors; the trajectory would then follow the linac centerline and the dispersive error would be zero.

Obviously, this is not realistic. When the additional errors are included, the difference orbit is not a function of just $\left(N_{y}+2\right)$ unknowns. Thus, we cannot calculate the individual quadrupole mi.aligmments and the initial conditions exactly. The error in each calculated value will increase with the number of misalignments being estimated since each calculation depends upon the accuracy of the preceding calcuiations. Of course, while the error of each individual calculation may be large, the global solution could be used to reduce the difference orbit to the level of the $\mathrm{BPM}$ precision errors. 
Unfortunately, if we use this global solution to correct the difference orbit, and thereby the dispersive error, we would find that the trajectory diverges from the linac centerline. This occurs because the difference orbit is not referenced to the linac centerline and small errors add. Thus, we need to include some information about the real trajectory while correcting the difference orbit.

We have found that the best approach is to perform a least squares solution for the unknowns, using both the original trajectory and the difference orbit weighted with the absolute accuracy with which these trajectories are known. Thus, we solve for the dipole corrector strengths which minimize the sum

$$
\sum_{j \in\{B P M I\}} \frac{\left(m_{j}+X_{j}\right)^{2}}{\sigma_{\text {prec }}^{2}+\sigma_{\text {BPM }}^{2}}+\frac{\left(\Delta m_{j}+\Delta X_{j}\right)^{2}}{2 \sigma_{\text {prec }}^{2}}
$$

where $\sigma_{\text {prec }}$ is the rms of the BPM precision errors and $\sigma_{\mathrm{BPM}}$ is the rms of the $\mathrm{BPM}$ misalignments relative to the linac centerline. In addition, $X_{j}$ is the predicted trajectory at the $j$ th $\mathrm{BPM}$ as a function of the dipole corrector strengths and $\Delta X_{j}$ is the predicted difference orbit. We will subsequently refer to this algorithm as Dispersion-Free (DF) correction.

\subsubsection{DF Error Analysis}

In this section, we analyze the effect of various errors on the performance of the DF correction algorithm. In a linac, there are many additional errors that were not included in the initial formulation of the DF algorithm. Thus, to understand the utility of the algorithm, we have to determine its sensitivity to these additional errors.

The DF algorithm corrects the dispersive error by correcting a measured difference orbit which is created by changing the effective beam energy. Thus, the algorithm relies upon the resemblance between this measured difference orbit and the actual dispersive error. We can divide any errors into two catagories: errors which cause the measured difference orbit to differ from the actual difference orbit, (measurement errors) and errors which cause the difference orbit to differ from the dispersive error of a particle within the beam.

Errors in neither category will not degrade the correction of the chromatic dilution and thus can be ignored. This is an important aspect of the algorithm. The algorithm does not attempt to extract specific information from noisy data; it 
minimizes a measured quantity. Thus, if errors reduce the convergence of the minimization, one can simply iterate the correction procedure. Examples of such errors are BPM and corrector calibration errors, absolute beam energy errors, and errors in the transport coefficients $R_{12}$ used to calculate the corrections. These errors will only slow the convergence of the algorithms. Rather than requiring one iteration, perhaps two or even three iterations will be required to achieve a good solution.

In contrast, errors in the first two catagories will cause the algorithm to converge to an incorrect solution. BPM precision and beam jitter errors are examples of errors from the first category. Magnet scaling errors, RF deflections, and effects due to the nonlinearity of the dispersion are examples of errors in the second category. We will first discuss effects due to the nonlinearity of the dispersive error and then we will discuss each of the other effects in turn. We proceed in this order since the nonlinearity is important for determining the full effect of the BPM precision and beam jitter errors.

\subsubsection{NONLINEARITY}

The goal of our correction algorithm is to improve the transverse emittance dilution due to the dispersive error; we want to correct the dispersive error arising from the energy variation within the particle bunch. The dispersion is a nonlinear function of the energy deviation and thus, ideally, we would like to measure the difference orbit by making an effective energy change comparable to the bunch energy spread. Unfortunately, this tends to be small, the order of $1 \%$ or less, and we will see that a small effective energy' change can lead to large errors in the corrected solution. Therefore, we need to determine the effect of correcting a difference orbit created by an energy change that is substantially different from the beam energy spread.

The equation for the difference orbit $\Delta y_{\delta}=y_{c}-y_{\delta}$, due to an energy difference of. $\delta$, is equal to the equation for the dispersive error $\mathrm{Eq}$. (3.4.1), found in Section 3.4.1. Neglecting the adiabatic damping, this can be written:

$$
\Delta y_{\delta}^{\prime \prime}+K_{1} \Delta y_{\delta}=\delta\left(G-K_{1} y_{q}+K_{1} y_{c}+K_{1} \Delta y_{\delta}\right)
$$

where $y_{q}$ and $y_{c}$ are the quadrupole misalignment and the on-energy trajectory and, here, we have used the notation $\Delta y \delta$ instead of $\Delta y_{d}$; the subscript $\delta$ refers to the magnitude of the energy change which created the difference orbit. W'e solve 
this equation perturbatively, treating all elements as infinitely thin in length, i.e., delta-functions in $s$. To second order, this yields

$$
\Delta y_{\hat{\delta}}(s)=\Delta y_{\hat{\delta}}^{(1)}(s)+\sum_{\boldsymbol{i}} \delta_{\boldsymbol{i}}\left(K_{1} L_{q}\right)_{i} \Delta y_{\delta}^{(1)}\left(s_{\boldsymbol{i}}\right) R_{12}\left(s, s_{\boldsymbol{i}}\right)
$$

where the first order term is

$$
\Delta y_{\delta}^{(1)}(s)=\sum_{i} \delta_{i}\left(\left(G^{\prime} L\right)_{i}-\left(y_{c i}-y_{q i}\right)\left(K_{1} L_{q}\right)_{i}\right) R_{12}\left(s, s_{i}\right)
$$

Here, $y_{c i}$ and $y_{q i}$ are the on-energy trajectory and misalignment at the $i$ th quadrupole, and $G L$ and $K_{1} L_{q}$ are the integrated deflection due to a dipole field and the integrated quadrupole strength, respectively.

Equation (3.5.5) illustrates a potential problem of using an energy change $\delta$ that is substantially larger than the beam energy spread $\sigma(s)$. Because the dispersive error is a nonlinear function of the energy deviation, if $\delta$ is relatively large, it is possible to correct the resulting difference orbit $\Delta y_{\delta}$ to a small value while having a large first order contribution $\Delta y_{\delta}^{(1)}$; the second order contribution can be used to cancel the first order term. Unfortunately, this cancellation does not work within the beam since the beam energy spread is small. Thus, one can correct $\Delta y_{6}$ while actually increasing the dispersive dilution of the beam. In the subsequent sections, we will use Eqs. (3.5.5) and (3.5.6) to determine the effect of errors on the DF correction algorithm.

\subsubsection{BPM PRECISION}

The BPM precision errors will limit the accuracy with which we can correct the difference orbit. Assuming that the BPMs have random precision errors with an rms of $\sigma_{\text {prec }}$, the measured difference orbit will differ from the actual difference orbit by an rms error of $\sqrt{2} \sigma_{\text {prec }}$. We can estimate the residual dispersive error, after correcting this measured difference orbit, by considering a simplified example where one zeros the measured difference orbit at each of the BPMs. After correction, the actual difference orbit $\Delta y / \delta$ would be equal to the negative of the BPM precision errors.

Given this residual difference orbit $\Delta y_{\delta}$, we want to solve for the difference orbit $\Delta y_{\sigma}$ created by an energy change of $\sigma(s)$, where $\sigma$ is the rms energy spread. This 
will allow us to determine the emittance dilution due to the BPM precision errors using Eqs. (3.4.3) and (3.4.4). First, we use Eq. (3.5.5), with the change $\delta \rightarrow \sigma$, to express $\Delta y_{\sigma}$,

$$
\Delta y_{\sigma}=\Delta y_{\sigma}^{(1)}
$$

where we have only included the first order term since $\sigma$ is assumed small. Next, we invert Eq. (3.5.5) to solve for $\Delta y_{\delta}^{(1)}$ from the residual difference orbit $\Delta y_{\delta}$

$$
\Delta y_{\delta}^{(1)}(s)=\Delta y_{\delta}(s)-\sum_{i} \delta_{i}\left(K_{1} L_{q}\right)_{i} \Delta y_{\delta}\left(s_{i}\right) R_{12}\left(s, s_{i}\right)
$$

Now, we need to relate $\Delta y_{\sigma}^{(1)}$ to $\Delta y_{\delta}^{(1)}$. The difference orbit $\Delta y_{\delta}$ is equal to the negative of the random BPM precision errors; it is constructed from sums of "deflections" and coefficients $R_{12}$ which oscillate, Eqs. (3.5.5) and (3.5.6). Thus, there are three length scales we need to consider: the length between "deflections" $L_{D}$, the betatron oscillation period $L_{\beta}$, and the length over which $\sigma(s)$ changes $L_{\sigma}$. Provided that $L_{D}, L_{\beta} \ll L_{\sigma}$, we can treat $\sigma$ and $\delta$ as constant over the correlated "deflections". This allows us, using Eq. (3.5.6) and performing ensemble averages over the random deflections, to find the relation:

$$
\left\langle\Delta y_{\sigma}^{(1)^{2}}\right\rangle=\frac{\overline{\sigma^{2}}}{\delta^{2}}\left\langle\Delta y_{\delta}^{(1)^{2}}\right\rangle
$$

where $\overline{\sigma^{2}}$ is the average of $\sigma^{2}(s)$ over $s$. Now, using this result and Eqs. (3.5.7) and (3.5.8), we find that after DF correction the BPM precision errors cause an rms residual dispersive error of

$$
\left\langle\Delta y_{\sigma \mathrm{B} P M}^{2}\left(s_{f}\right)\right\rangle=2 \sigma_{\text {prec }}^{2} \frac{\overline{\sigma^{2}}}{\delta^{2}}+2 \sigma_{\text {prec }}^{2} \overline{\sigma^{2}} \sum_{i}\left(K_{1} L_{q}\right)_{i}^{?} R_{12}^{2}\left(s_{f}, s_{i}\right) .
$$

To evaluate the sum in the second term in $\mathrm{Eq}_{q}$. (3.5.10), we need to assurne a model linac. We use the NLC: model described Appendix D, where the phase advance per cell is constant while the beta functions and the cell lengths increase with the square root of the beam energy. In adclition. Wo assume that the moncorelated energy spread of the beam decreases inversely with the bean encrgy. Using this model, we 
can evaluate the sum in Eq. (3.5.10) by approximating it with an integral. In this manner, we find an expected dispersive error due to the BPM precision errors of

$$
\left\langle\Delta y_{\sigma_{\mathrm{BPM}}^{2}}^{2}\left(s_{f}\right)\right\rangle \approx 2 \sigma_{\mathrm{prec}}^{2} \frac{\sigma_{0}^{2}}{\delta^{2}} \frac{\gamma_{0}}{\gamma_{f}}+\frac{1}{2} \sigma_{\mathrm{prec}}^{2} N_{\mathrm{BPM}}{\overline{\beta_{0}}}^{2}\left(K_{1} L_{q}\right)_{0}^{2} \sigma_{0}^{2} \frac{\gamma_{0}}{\gamma_{f}}
$$

Here, $\overline{\beta_{0}},\left(K_{1} L_{q}\right)_{0}$, and $\sigma_{0}$ are the average beta function, the integrated quadrupole strength, and the energy spread at the beginning of the linac.

Finally, we can use Eqs. (3.4.3) and (3.4.4) to solve for the dispersive emittance dilution due to this error. We will not perform the explicit substitutions here, but we will instead discuss a few implications of Eq. (3.5.11). First, Eq. (3.5.11) indicates that there will be a minimum dispersive error, due to the BPM precision errors, for some value of $\delta$. The first term of Eq. (3.5.11) decreases rapidly with $\delta$ while the second term remains constant; if we had included higher orders of $\delta$, we would find terms that increase with $\delta$. Secondly, notice that both terms in Eq. (3.5.11) can be reduced by decreasing $\sigma_{\text {prec }}$, i.e., the measurement error; the rms of the BPM precision errors will tend to decrease with the square root of the number of measurements when the separate measurements are averaged together. We will see this behavior in the results of simulations discussed in Section 3.5.4.

\subsubsection{BEAM JiTTER}

In a linac there are many sources of jitter which cause the beam position to fluctuate. For example, injection jitter, ground motion, and power supply fluctuations all have this effect. To prevent these from degrading the performance of a linear collider, this beam jitter must be constrained to be much less than the beam size; the beam jitter will directly increase the time average of the emittance since the beam changes position with time, thereby, increasing the effective phase-space volume occupied. In this section, we will estimate a secondary effect of the beam jitter, namely, its effect on the performance of the DF correction algorithm.

The beam is most sensitive to dispersive errors when the beam energy spread is large and thus we will only' consicler jitter of the injected beam; the beam energy spread is typically largest at injection. Of course, if desired, our result could easily be generalized by summing over all the sources of the jitter. In fact, this will be done when analyzing the effect of magnet scaling errors which are treated in an analogous manner to the beam jitter errors. 
In the case of injection jitter, the measured difference orbit will differ from the actual difference orbit by a betatron oscillation caused by the jitter in initial conditions. After correcting the measured difference orbit to zero, the actual difference orbit $\Delta y_{\delta}$ will be the negative of this betatron oscillation. To estimate the magnitude of $\Delta y_{\sigma}$, we use Eqs. (3.5.7) and (3.5.8) derived in the previous section on the BPM precision errors. This case differs from the case of the BPM precision errors in that the deflections that create the difference orbit, i.e., the summand of Eq. (3.5.6), are only non-zero near the beginning of the linac. Thus, instead of Eq. (3.5.9), we have the relation

$$
\Delta y_{\sigma}^{(1)}=\frac{\sigma_{0}}{\delta} \Delta y_{\delta}^{(1)}
$$

where $\sigma_{0}$ is the rms energy spread at the beginning of the linac. Using this and Eqs. (3.5.7) and (3.5.8), we find an expected dispersive error due to the beam jitter of

$$
\begin{array}{r}
\left\langle\Delta y_{\sigma \mathrm{jit}}^{2}\left(s_{f}\right)\right\rangle=2 \sigma_{\mathrm{jit}}^{2} \frac{\sigma_{0}^{2}}{\delta^{2}} \sqrt{\frac{\gamma_{0}}{\gamma_{f}}}+\left\langle\left(\sum _ { i } \sigma _ { 0 } ( \Lambda _ { 1 } L _ { q } ) _ { i } R _ { 1 2 } ( s _ { f } , s _ { i } ) \left[ y_{0} R_{11}\left(s_{i}, s_{0}\right)\right.\right.\right. \\
\left.\left.\left.+y_{\delta} R_{11}\left(\delta ; s_{i}, s_{0}\right)\right]\right)^{2}\right\rangle
\end{array}
$$

where $\sigma_{\mathrm{jit}}$ is the rms jitter measured at the beginning of the linac and the factor of two appears in the first term since two trajectories must be measured to calculate a difference orbit. The angle brackets denote an ensemble average over the jitter. This is represented by $y_{0}$ for jitter of the on-energy trajectory and $y_{\delta}$ for jitter of the trajectory after making the energy change of $\delta$; note that for simplicity we have only included position jitter in these terms.

The expression in Eq. (3.5.13) differs from the expression found for the BPM precision errors, Eq. (3.5.10). The BPM precision errors are uncorrelated and thus the error $\left\langle\Delta y_{\sigma}{ }^{2}\right\rangle$ depends upon $N_{\text {BPM. }}$. In contrast, beam jitter leads to a difference orbit that oscillates like a betatron oscillation. Thus, the error due to the nonlinearity, the second term of Eq. (3.5.13), will be correlated with the betatron phase advance and $\left\langle\Delta y_{\sigma}{ }^{2}\right\rangle$ will depend upon the square of $N_{q}^{\prime}$.

When performing the sums in $\mathrm{E}(\mathrm{y}$. (3.5.13), we treat jitter of the on-energy trajectory and the off-energy trajectory separately because the induced betatron oscillations will have different phase advances; the phase advance is energy dependent. In particular, after changing the effective beam energy, the different phase advance 
will cause the correlation between the injection error and the dispersive error to breakdown. Thus, we would find that jitter of the off-energy trajectory contributes far less than jitter of the on-energy trajectory; for this reason, we will only estimate the on-energy jitter.

Using the NLC model linac, we can evaluate the sums in Eq. (3.5.13) by approximating them with integrals. After DF correction, we find an expected dispersive error due to beam jitter of

$$
\left\langle\Delta y_{\sigma_{\mathrm{jit}}^{2}}^{2}\left(s_{f}\right)\right\rangle \approx 2 \sigma_{\mathrm{jit}}^{2} \frac{\sigma_{0}^{2}}{\delta^{2}}+4 N_{\mathrm{cell}}^{2} \sigma_{\mathrm{jit}}^{2} \sigma_{0}^{2} \tan ^{2} \psi_{c} / 2 \sqrt{\frac{\gamma_{0}}{\gamma_{f}}}
$$

where we have simplified the expression with Eq. (D.1.3).

Again, one can solve for the dispersive emittance dilution due to this error using Eqs. (3.4.3) and (3.4.4). As in the discussion of the BPM precision errors, we will not perform the explicit substitutions here, but instead we will discuss Eq. (3.5.14). Similar to the error due to the BPM precision errors, the first term of Eq. (3.5.14) decreases rapidly with $\delta$ while the second does not, indicating that the residual error due to the beam jitter will have a minimum as a function of $\delta$. Also notice that, like the error due to the BPM precision errors, this contribution depends upon the measurement errors, and thus it can be reduced by averaging multiple measurements of the trajectory. We will estimate the effects of the beam jitter at the end of Section 3.5.4, after discussing results from the simulations.

\subsubsection{Magnetic Scaling Errors}

Magnetic scaling errors occur when one changes the effective beam energy by scaling the magnetic fields. The errors arise because different magnets will scale slightly differently with the power supplies and because the power supplies have finite precision. Thus, one cannot reduce all of the magnetic fields by exactly the same percentage. Typically, it is possible to specify the magnetic field strength with an accuracy of roughly $10^{-3}$.

The effect of these scaling errors is analogous to the effect of beam jitter. When measuring a difference orbit created by scaling the magnetic fields, one changes the fields, measures the off-energy trajectory, resets the magnets and measures the onenergy trajectory. We treat the final magnet values as the reference values and thus the scaling errors only cause error's when measuring the off-energy trajectory. We 
should emphasize that the order in which one measures the trajectories is important. If we measure the on-energy trajectory before the off-energy trajectory, the scaling errors would cause errors in both measurements.

If a magnet deflects the central trajectory by an angle $\theta$ and its magnetic field can be specified with an rms accuracy of $\Delta$, the off-energy trajectory will have an rms deflection error of $(1-\delta) \Delta \theta$. These deflections will drive betatron oscillations which will add to the measured difference orbit just as beam jitter does. After correction with the DF algorithm, the actual difference orbit will be corrected to the negative of this (off-energy) betatron oscillation. Thus,

$$
\Delta y_{\delta}(s)=-\sum_{i} \Delta_{i} \theta_{i} R_{12}\left(\delta ; s, s_{i}\right)
$$

where we have neglected the factor $(1-\delta)$. Now, since the deflections $\Delta \theta$ are random, we can use Eqs. (3.5.7), (3.5.8), and (3.5.9) to find $\Delta y_{\sigma}$. Using the NLC model linac, we find a residual dispersive error of

$$
\begin{aligned}
\left\langle\Delta y_{\sigma_{\text {magnet }}}^{2}\left(s_{f}\right)\right\rangle & \approx N \frac{\left\langle\Delta^{2}\right\rangle\left\langle 0_{0}^{2}\right\rangle}{\delta^{2}} \frac{\sigma_{0}^{2} \beta_{0}^{2}}{3} \frac{\gamma_{0}}{\gamma_{f}} \\
+ & \left\langle\left(\sum_{i} \sigma_{i}\left(K_{1} L_{q}\right)_{i} R_{12}\left(s_{f}, s_{i}\right) \sum_{j}^{i} \Delta_{j} \theta_{j} R_{12}\left(\delta ; s_{i}, s_{j}\right)\right)^{2}\right\rangle .
\end{aligned}
$$

Here, $N$ is the number of magnets and $O_{0}$ is the deflection due to the magnets at the beginning of the linac. In addition, we have assumed that the deflection errors $\theta$ are random and that they decrease in strength inversely with the square root of the beam energy; this models quaclrupole scaling errors in our example linac. In the case of quadrupole errors, $\theta$ is proportional to the integrated strength of the quadrupole and the distance of the trajectory from the magnetic center. Thus,

$$
\left\langle\theta_{0}^{2}\right\rangle=\left(\Lambda_{1}^{\prime} L_{q}\right)_{0}^{2}\left(\left\langle y_{m}^{2}\right\rangle+\left\langle y_{c}^{2}\right\rangle\right)
$$

where $y_{m}$ and $y_{c}$ are the rms values of the quadrupole misalignments and the boam trajectory. Alternately, for dipole correctors, 0 can be estimated by assuming a uniform distribution of kicks. Thus, $\left\langle 0_{0}^{2}\right\rangle$ is equal to one-third of the initial peak deflection. 
It is important to note that as described the magnet scaling errors only contribute to the error of the off-energy trajectory. The nonlinear term, i.e., the second term of $\mathrm{Eq}$. (3.5.16), will be small because the off-energy phase advance differs from the on-energy phase advance and thus we can neglect it. A similar situation occurs in the case of beam jitter errors as discussed in the previous section. Of course, if the difference orbit is not measured using the procedure described at the beginning of this section, the magnet scaling errors could also affect the on-energy trajectory. In this case, Eq. (3.5.16) would have a term proportional to $N \times N_{\text {quad }}^{2}$; obviously this should be avoided.

Finally, we should note that the effect of the magnetic scaling errors does not depend upon measurement errors which can be reduced. Thus, these errors could limit the performance of our correction algorithm. In Section 3.5.4, we will use Eq. (3.5.16) along with Eqs. (3.4.3) and (3.4.4) to estimate the magnitude of the errors; fortunately, they cause only a small error.

\subsubsection{RF DEFLECTIONS}

The RF is provided to accelerate the particles longitudinally. As discussed in Section 3.4.3, there is typically a small coupling between the accelerating field and the transverse planes. The RF deflections present a problem for our algorithm because, unlike magnetic deflections, the RF deflections remain constant as the effective beam energy is changed. Thus, they cause the measured diffcrence orbit to differ from the actual dispersive error.

As expressed in Section 3.4.3, the RF deflections are a sinusoidal function of the RF phase, typically offset in phase relative to the longitudinal acceleration:

$$
\theta_{\mathrm{RF}}=g \frac{\gamma_{\mathrm{RF}}}{\gamma^{\prime}(s)} \sin \left(z k_{\mathrm{RF}}+\phi_{0}\right)
$$

Here, $g$ is the longitudinal-transverse coupling of the accelerating structure, $\gamma_{R F}$ is the energy gain from the structure, and $\gamma(s)$ is the beam encrgy. In addition, $\phi_{0}$ is the phase of the deflection relative to the bunch.

There are two methods of changing the effective beam energy when measuring the difference orbit: one can either change thr magnet strengths or the actual beam energy. For either method to work properly, all of the deflections should scale with the change in effective energy. Infortunately, if one changes the magnet strengths, 
the RF deflections are not affected. Likewise, if one scales the RF to change the beam energy, the RF deflections also scale and the effect on the beam does not change.

Since the RF deflections do not change when varying the effective beam energy, the measured difference orbit will be independent of the RF deflectlons. The dispersive error in the beam resulting from the RF deflections and the beam energy spread can be directly calculated from $\mathrm{Eq}$. (3.5.5) with the substitution $\delta \rightarrow \sigma(s)$. Assuming that the RF errors are random and using the same model linac described earlier, we find a residual dispersive el'ror of

$$
\left\langle\Delta y_{\sigma \mathrm{RF}}^{2}\left(s_{f}\right)\right\rangle \approx \frac{N_{\mathrm{RF}}}{3}\left\langle g^{2} \cos ^{2}\left(\phi_{0}-\phi_{d}\right)\right\rangle \sigma_{0}^{2} \beta_{0}^{2} \frac{E_{\mathrm{RF}}^{2}}{E_{f} E_{0}} \sqrt{\frac{E_{0}}{E_{f}}} .
$$

Here, $N_{\mathrm{RF}}$ is the number of acceleratol sections.

Notice that the RF errors do not depend upon the effective energy change $\delta$ used to create the difference orbit. Furthermore, this effect cannot be reduced by making multiple measurements of the trajectory. Thus, the RF errors could provide a serious limitation on the performance of the correction algorithm. We will use $\mathrm{Eq}$. (3.5.19) along with Eqs. (3.4.3) and (3.1.4) to estimate the importance of these errors in both the SLC and NLC linacs aften describing the results of the simulations in Section 3.5.4.

\subsubsection{DF Simulations}

A computer program was written to test the DF correction technique against the one-to-one correction algorithm. The program simulates random transverse misalignments of the quadrupoles and BPMs, random quadrupole strength errors, and BPM precision errors. The effects of RF deflections and beam jitter have not been directly included; these are discussed at the end of this section using the results of Section 3.5.3. Finally, the effect of the wakefields are neglected; these are discussed in the section describing the W'F technique.

All of the simulations were performed on the 250 GeV NLC linac described in Appendix D. To simulate correcting the orbit in the NLC, we use twenty different sets of random errors. The errors are found from gaussian distributions which had been cutofr at two sigma. The guadrupoles are misaligned $70 \mu \mathrm{m}$ rms relative to 
the linac centerline and the BPMs are misallgned $70 \mu \mathrm{m}$ rms relative to the quadrupoles; thus, the BPMs are misaligned with an $\mathrm{m}$ (ms of roughly $100 \mu \mathrm{m}$ relative to the linac centerline. Furthermore, BPM precision errors of $2 \mu \mathrm{m}$ are assumed since a measurement precision the order of the beam size will need to be achieved in the NLC. Finally, 0.1\% rms quadrupole strength errors are also included, simulating calibration errors; the errors do not change from measurement to measurement as power supply fluctuations would.

In the NLC, the trajectory is assumed to be corrected by moving the quadrupoles horizontally or vertically to achieve the desired deflection. Both the focusing and defocusing quadrupoles are used for correction. When correcting with the DF algorithm, the linac is divided into eleven sections, each containing twenty cells, The algorithm is then used to correct each section instead of correcting the entire linac at once. While correcting the linac in sections will not minimize the dispersion as well as correcting the entire linac at once, this procedure reduces the sensitivity to discrepancies between the machine and the model one uses for correction. In all cases, the solutions are calculated with a single iteration of the DF algorithm.

Table 12. Results of DF simulations in the $250 \mathrm{GeV}$ NLC linac.

\begin{tabular}{|c|c|c|}
\hline & $1-$ to- 1 & DF \\
\hline Orbit rms & $89 \pm 1 \mu \mathrm{m}$ & $54 \pm 1 \mu \mathrm{m}$ \\
\hline BPM rms & $3 \pm 0.1 \mu \mathrm{m}$ & $80 \pm 1 \mu \mathrm{m}$ \\
\hline Dilution $\Delta \epsilon_{y}$ & $0.20 \pm 0.8 \epsilon_{y 0}$ & $0.02 \pm 0.01 \epsilon_{y 0}$ \\
\hline
\end{tabular}

Results from correcting the twenty sets of errors with the two correction schemes are listed in Table 12; the error on the data is equal to one standard error. The Orbit rms data is the rms of the trajectory relative to the linac centerline, while the BPM rms data is the rms of the BPM measurements. Notice that the one-to-one algorithm zeros the BPM readings (within the BPM precision) while the actual trajectory is relatively large. In contrast, our method corrects both the actual trajectory and the measured BPM readings. In fact, the DF correction algorithm does better correcting the actual trajectory than does the one-to-one method.

Of course, we are interested in more than just correction of the trajectory. The dilution of the vertical emittance due to the dispersive errors is listed in the bottom row of Table 12; the dilution of lle horizontal emittance will be much smaller than 

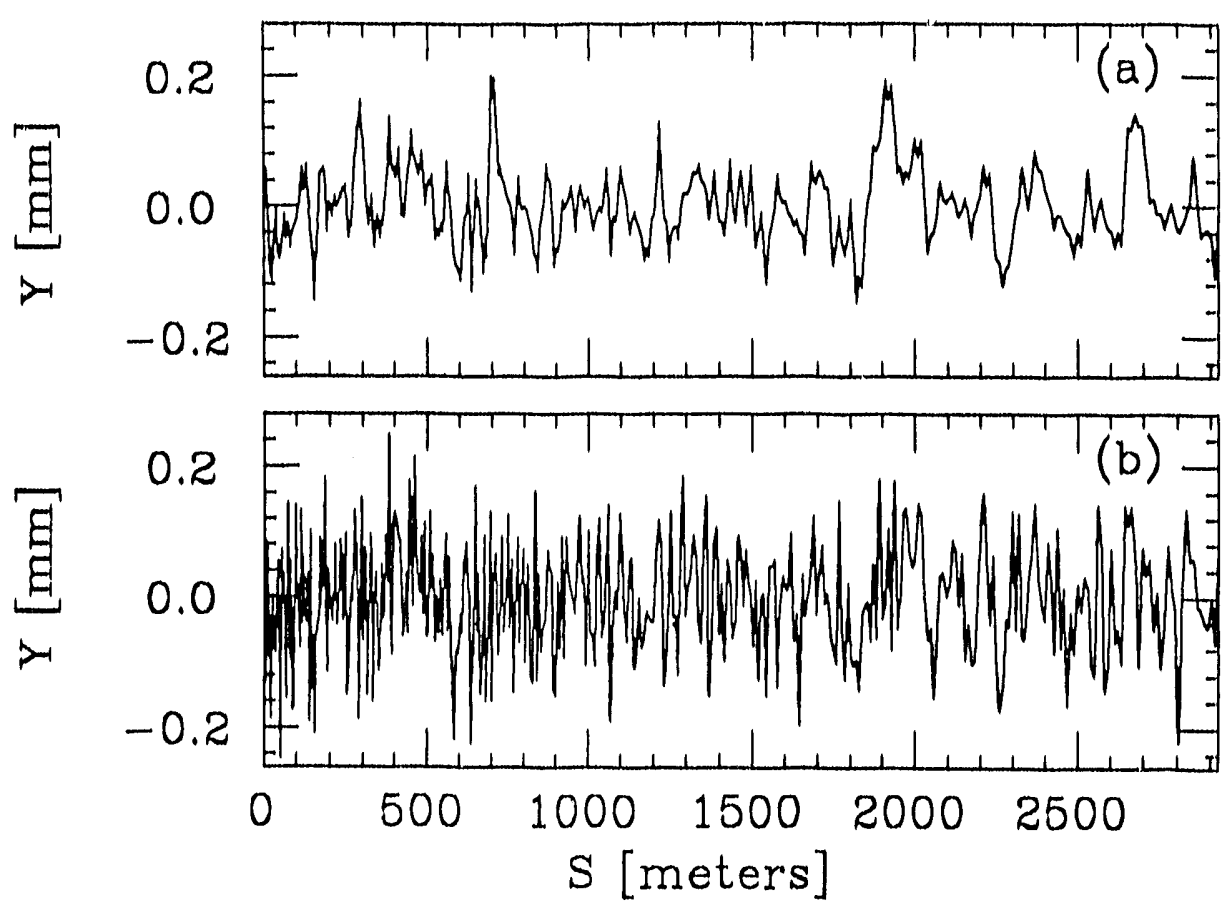

Fig. 29 Actual trajectory in the NLC after (a) DF correction and (b) 1-to-1 correction.
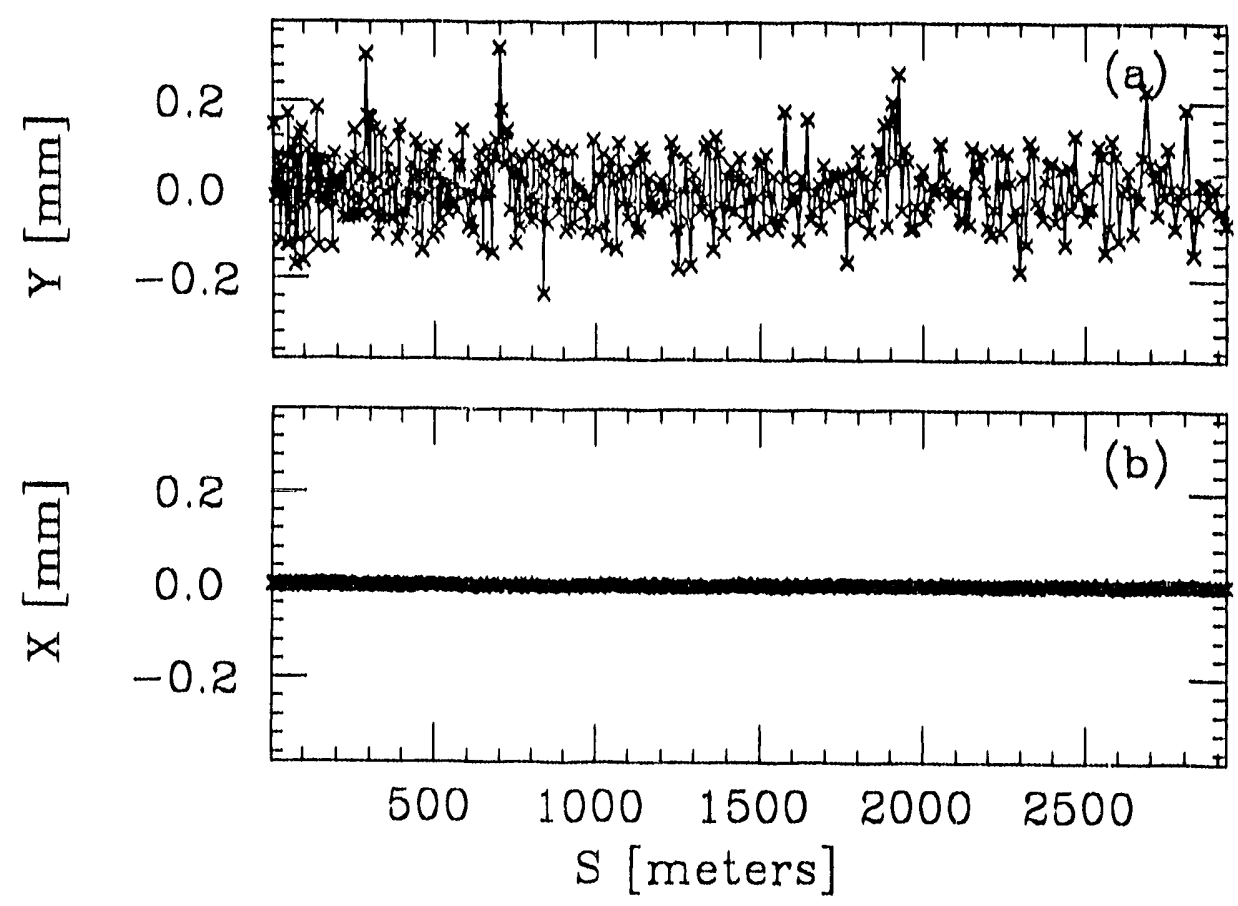

Fig. 30 BPM measurements in the NLC after (a) DF correction and (b) 1-to-1 correction. 

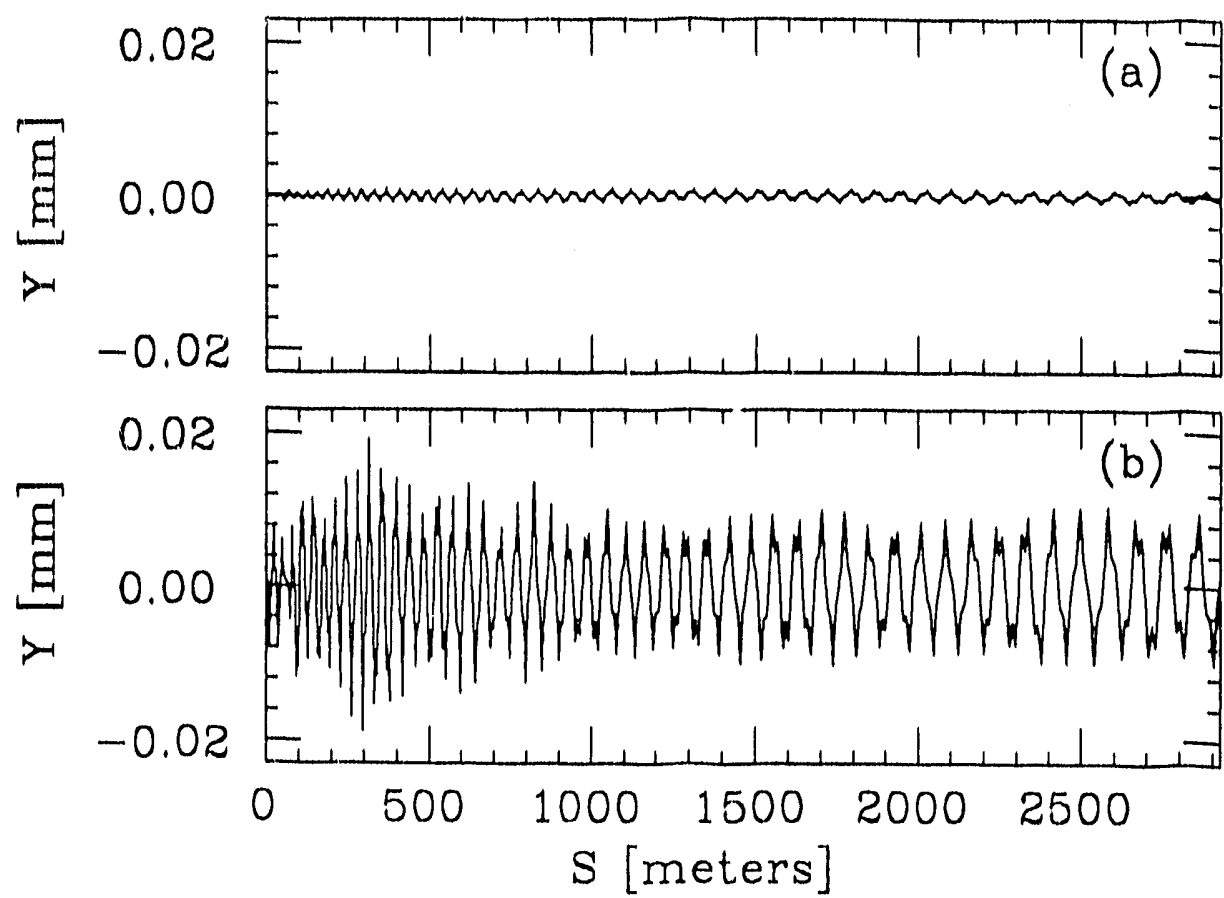

Fig. 31 Difference orbit in the NLC after (a) DF correction and (b) 1-to-1 correction.

the vertical since the initial emittance is one hundred times larger. Obviously, the one-to-one correction technique leads to a large (factor of six) increase in the vertical emittance. Furthermore, this emittance will continue to filament and thus the effective emittance growth may be much larger. In contrast, the new technique performs very well, virtually eliminating the dispersive dilution.

The difference in correction techniques is illustrated in Figs. 29-33. Figure 29 compares the trajectory after DF correction (upper plot) with the result of one-toone correction (lower plot) and Fig. 30 compares the BPM readings in the same manner. One can see that the one-to-one method zeroes the BPMs, but does not correct the actual trajectory as well as the DF method. Finally, Fig. 31 shows the difference between the trajectory of an on-energy particle and a particle whose energy differs from the design, the energy difference being equal to the rms uncorrelated energy spread which is $1 \%$ at injection and decays inversely with energy. Obviously, the dispersive error, i.e., this difference orbit, and therefore the dispersive dilution, is much smaller in the case of the DF correction.

Figure 32 is a plot of the $y-y^{\prime}$ phase-space at the end of the NLC linac after correction with the one-to-one algorithm. The curve plots the endpoints of particle trajectories having energies between $+\sigma_{t}$ and $-\sigma_{t}$. Also, for reference, the RMS 


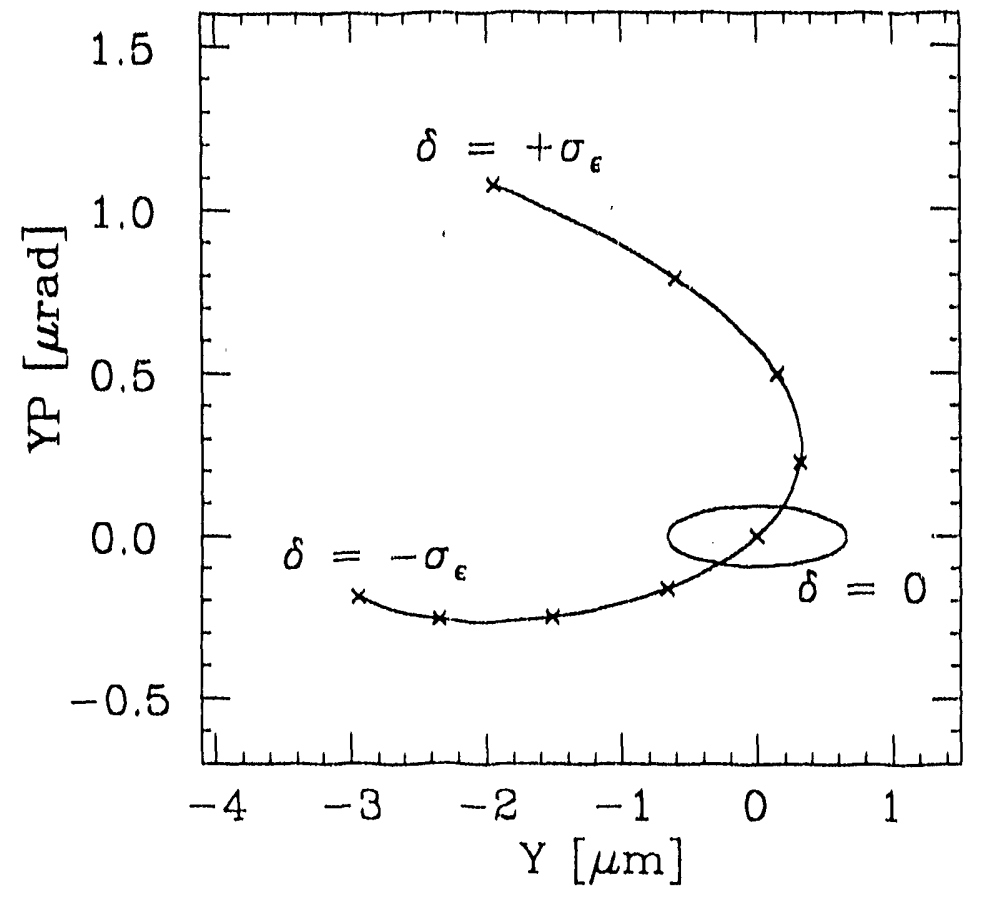

Fig. $32 y-y^{\prime}$ phase-space at the end of the 250 GeV NLC linac after one-toone correction; the points are the centroids of the beam slices with $\delta=+\sigma_{\epsilon}$ to $-\sigma_{\epsilon}$ and the beam ellipse for $\delta=0$ is plotted for reference.

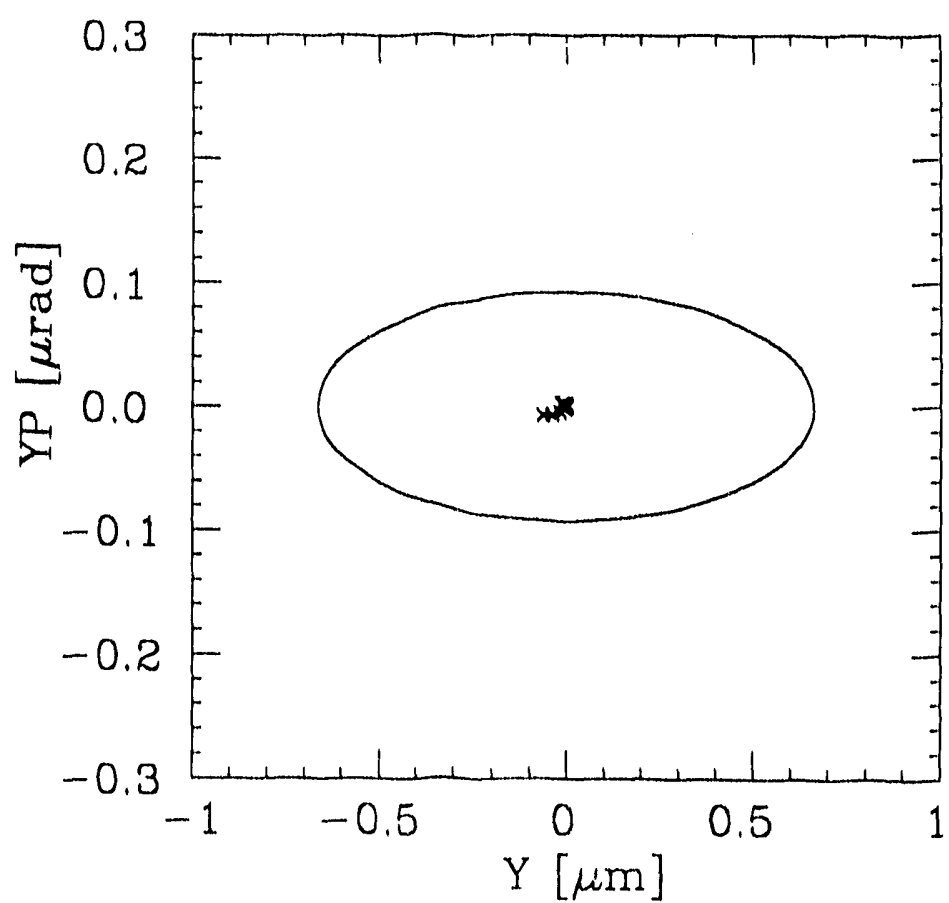

Fig. $33 y-p_{y}$ phase-space at the ond of the 250 GeV NLC linac after DF correction; the points are the centroids of the bean slices with $\delta=+\sigma_{t}$ to $-\sigma_{\epsilon}$. 
beam size, excluding the chromatic errors, is plotted about the design energy trajectory. Obviously, there is a large dispersive dilution in Fig. 32; the emittance dilution is roughly eight times the initial emittance. For comparison, we plot, in Fig. 33, the $y-y^{\prime}$ phase-space at the end of the NLC after DF correction. This is the same phase-space, although with different scales, as Fig. 32. After DF correction, the emittance dilution is quite small, roughly $1 \%$ in this case.

It is evident from Table 12 and Figs. 29-33 that the DF correction technique per. forms substantially better that the one-to-one method. In all of the data shown, the effective energy change used by the DF algorithm was $\Delta E / E=+10 \%$. Changing the beam energy, or equivalently changing the magnet strengths, is not necessarily easy and can in itself introduce error's. For this reason, we wish to limit the energy change used by the correction algorithm. Unfortunately, as the energy difference is decreased, the measurement of the difference orbit, used by the DF algorithm, will be lost in the noise of the BPM precision errors. Thus the correction technique will not perform as well.

In Fig. 34, we plot results of the DF correction technique, again found from the correction of twenty sets of random errors, versus the change in effective energy $\Delta .2 !$. There are three curves: the dotted is the emittance magnification which has a scale on the right, the solid is the rms of the actual trajectory, and the dashed curve is the rms of the BPM measurement of the trajectory. Notice that both the emittance magnification and the ms of the trajectory have broad minimums. The increase which occurs as $\Delta E / E$ increases is due to the nonlinearity of the dispersion. In contrast, as $\triangle E / E$ decreases, the $\mathrm{BPM}$ precision errors reduce the effectiveness of the algorithm. Our estimate Eq. (3.5.11) is in good agreement with both the behavior and the magnitude of this residual error. Finally, notice that the minimum in the residual emittance will shift towards larger $\Delta E / E$ when one includes other errors since the measurement resolution increases as $\Delta E / E$ increases.

Finally, in Fig. 35, we compare the result of the DF correction technique versus the magnitude of the BPM precision errors; the errors are varied from $2 \mu \mathrm{m}$ to $40 \mu \mathrm{m}$. As before, the data was found from the correction of twenty sets of random errors. As in Fig. 34, the three curves: solid, dashed, and dotted, are the actual trajectory rms, the measured trajectory rms, and the vertical emittance dilution; the first two curves have scales on the left and the emittance dilution has a scale on the right side of the plot. Notice that the emittance dilution is still less than $25 \%$ when the BPM precision errors have been increased to $8 \mu m$, which is roughly eight times the 


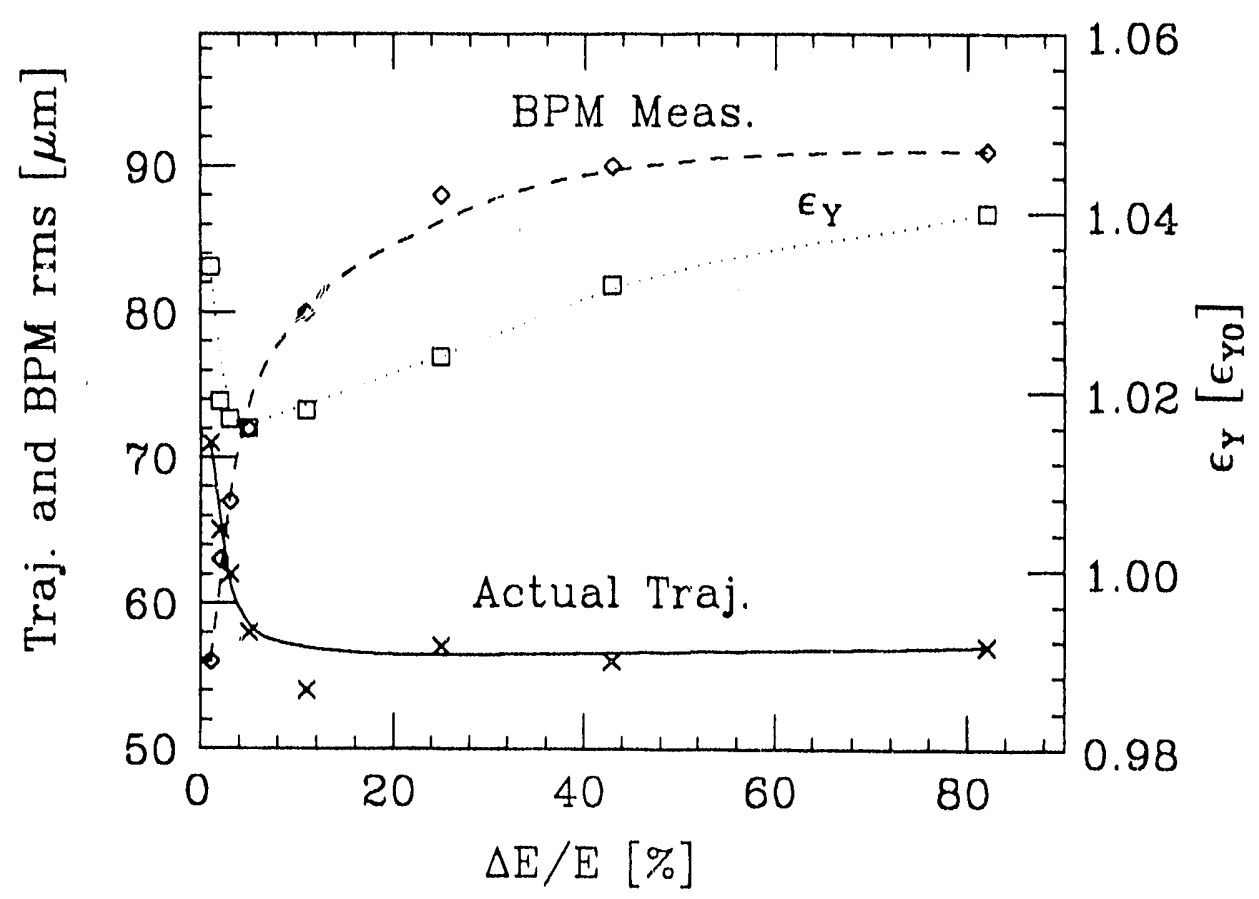

Fig. 34 DF correction versus the energy change $\Delta E / E$; the solid, dashed, and dotted lines are the actual trajectory, the measured trajectory, and the emittance after correction.

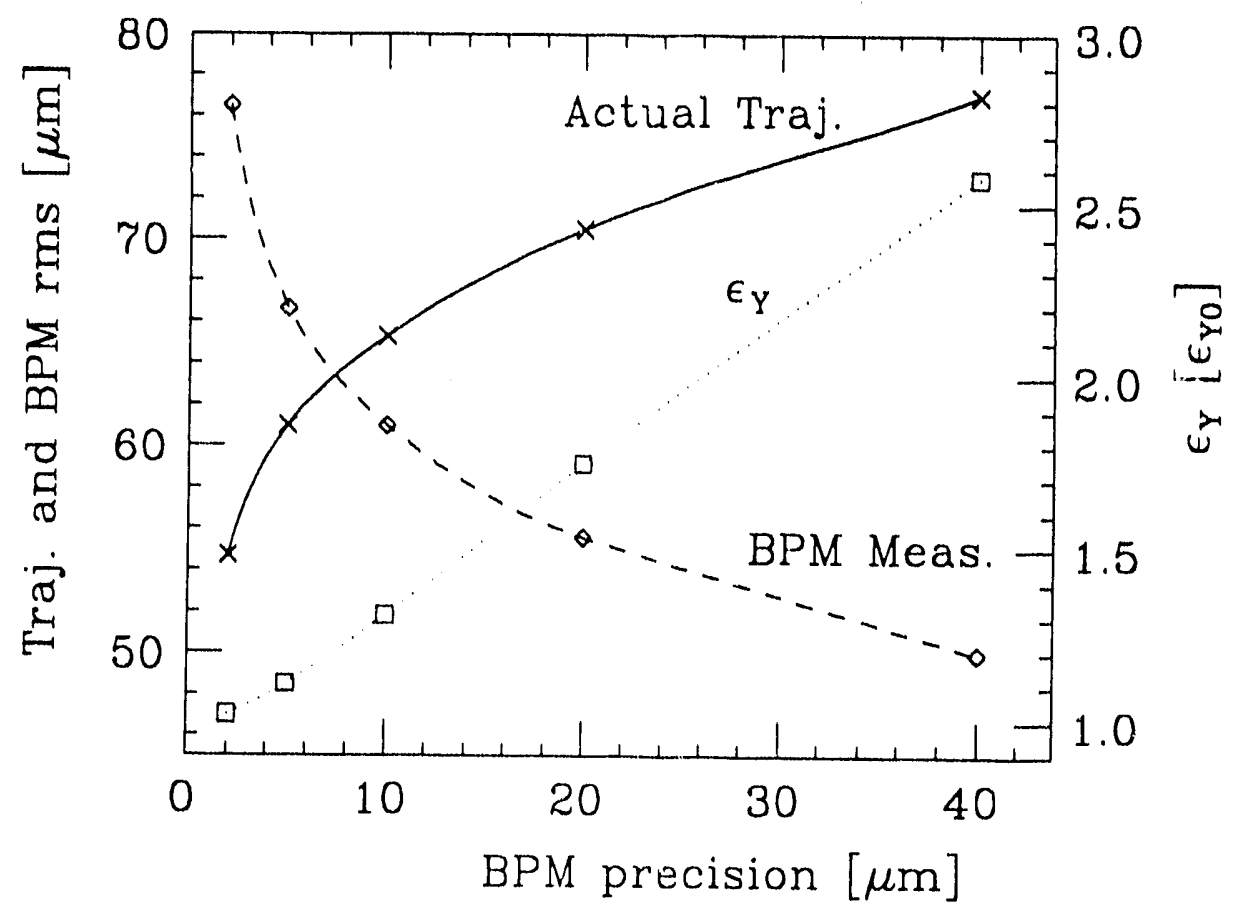

Fig. 35 DF correction versus the BPM precision errors; the solid, dashed, and dotted lines are the actual trajectory, the measured trajectory, and the emittance after correction. 
vertical beam size. Also notice that the rms of actual trajectory decreases rapidly as the magnitude of the BPM precision errors is decreased. In fact, the decrease in the trajectory rms, which is important for controlling wakefield effects, may be the most significant gain from decreasing the BPM precision errors.

\subsubsection{ERRORS}

In this section we estimate the effects of errors not included in the simulations. Specifically, we discuss the effect of beam jitter, RF deflections and magnetic scaling errors. In the NLC, we estimate the effect of the RF deflections using the same transverse-longitudinal coupling $g$ that was measured in the SLC: ${ }^{[108]} g=4 \times 10^{-4}$. We further assume that the accelerator sections in the NLC are one meter in length and each gives an exergy gain of $100 \mathrm{MeV}$. In this case, Eq. (3.5.19) yields an estimate of

$$
\Delta y_{\sigma \mathrm{RF}} \approx 0.3 \mu \mathrm{m} \quad(\mathrm{NLC})
$$

This residual dispersive error is comparable to the NLC vertical beam size. Using Eqs. (3.4.3) and (3.4.4), we find that it would lead to an emittance increase of roughly $5 \%$. Of course, as discussed in Section 3.4.3, the actual tolerances on the $\mathrm{RF}$ accelerator sections in the NLC need to be much tighter than those in the SLC. If the tolerance on the transverse-longitudinal coupling were reduced from $g=4 \times 10^{-4}$, this error should not be significant; the residual dilution scales with the square of $g$.

We also evtimate the effect of the magnetic scaling errors using random $1 \%$ absolute errors of the magnotic field strengths after scaling the magnets by $10 \%$. Using Eq. (3.5.16), we find

$$
\Delta y_{\sigma_{\text {Inagnet }}} \approx 0.5 \mu \mathrm{m}
$$

This causes a $13 \%$ emittance dilution. Of course, again, we believe that we have overestimated the error. In principle, one could reduce the absolute scaling error to $0.1 \%$ by cycling the magnets on a specified path through their hysteresis curves. In this case the dispersive dilution would be negligible.

Next, we consider the effect of beam jitter. Here, we estimate the effect of 
injection jitter that is $25 \%$ of the vertical beam size. Using Eq. (3.5.14), we find

$$
\Delta y_{\sigma_{\mathrm{jitter}}} \approx 0.3 \mu \mathrm{mn} \quad(\mathrm{NLC})
$$

when using an effeciive energy change of $10 \%$. This error causes an emittance dilution of $4 \%$.

Finally, to verify our estimates, we simulated both random magnetic scaling errors and jitter of the injected beam. Random $1 \%$ absolute errors were added to the magnetic fields strengths when scaling the magnets by $10 \%$. We found an emittance dilution of $10.0 \pm 2.7 \%$ due to the scaling errors; this is in fairly good agreement with our estimate of $13 \%$.

The injection jitter was simulated ly addling a random initial position offset to the trajectories used to measure the difference orbit; the position offsets have an rms equal to $25 \%$ of the initial beam size. In this case, we found an emittance dilution of $3.1 \pm 0.6 \%$ when making an effective energy change of $10 \%$. Again, this is in good agreement with our estimate of $4 \%$.

\subsubsection{WF Correction and Simulations}

Given the performance of the DF algorithm, we have ex ended its use to also correct wakefield dilutions that result from the corrected trajectory. In this section, we will describe the WF algorithm and then present the results of simulations. Because the WF technique is a simple extension of the DF method, this discussion will be brief. In particular, we will not reiterate the error analysis of Section 3.5.3; the analysis for the WF algorithm is very similar.

\subsubsection{WF ALGORITHM}

The goal is to find a new trajectory along which both the wakeficld and the dispersive effects cancel. The wakefields are caused by trajectory offsets in the accelerator sections whicn are due to both misalignments of the accelerator sections and a non-zero trajectory. If we ignore the accelerator misalignments, the effective offset in a section is just the average of the position in the two adjacent quadrupoles. By varying the quadrupole strengths in a specified manner, one can measure a difference orbit where the orbit in the quadrupoles will mimic the effects of the wakefields due to the trajectory. 
We can understand this by examining Eq. (3.4.17), derived in Section 3.4.2. Both the quadrupoles and the dipole wakefields cause a deflection that is proportional to the trajectory offset in the respective elements. But, the wakefield always deflects away from the structure centerline while the quadrupole deflection depends upon the sign of $K_{1}$. Assuming periodic FODO cells with accelerator sections centered between the quadrupoles, we find that, to mimic the wakefield effect, the quadrupole strengths must be varied as

$$
\frac{\delta K_{1}}{K_{1}} \propto \frac{L_{a c c}(s)}{\gamma(s) K_{1} L_{q}(s)} \sqrt{\frac{\bar{\beta}}{\beta_{q}}} \cos \Delta \psi
$$

where $\bar{\beta}$ and $\beta_{q}$ are the beta functions at the middle of the accelerator sections and the adjacent quadrupoles and $\Delta \psi$ is the betatron phase advance between the two. In addition, $L_{\mathrm{acc}}$ and $L_{y}$ are the lengths of the accelerator sections and the quadrupoles. Finally, note that because the correction is local, this condition can fluctuate slowly with $s$.

Condition (3.5.23) specifies that the quadrupole strength variation $\delta$ has opposite signs at focusing (QFs) and defocusing quadrupoles (QDs). In contrast, when creating the difference orbit to measure the dispersive error, $\delta$ has the same sign at both the QFs and the QDs. To correct both the wakefields and the dispersive errors one minimizes both of these difference orbits along with the actual trajectory. Unfortunately, it is not necessarily possible to increase some magnets while decreasing others since the quadrupoles are usually run close to their maximum strength. Thus, one can use an equivalent procedure where one minimizes a difference orbit created by varying only the QFs and a difference orbit created by varying only the QD magnets. In addition to being feasible, this later procedure also benefits from being simpler.

Strictly, by examining Eq. (3.4.17), we can see that minimizing these two difference orbits will reduce the wakefields if the accelerator sections are aligned to the centers of the quadrupoles, not the machine centerline. This can be remedied by varying the dipole correctors when varying the quadrupoles. Thus, the dipole correctors (partially) cancel the effect of the quadrupole misalignments; they must or the trajectory would tend to grow. In practice, the correction technique works best when the accelerator structures are aligned to the quadrupoles, but, as will be demonstrated, it still works very well when the accelerator sections are aligned to the ideal centerline. 
Table 13. Results of WF simulations in the $250 \mathrm{GeV}$ NLC linac.

\begin{tabular}{|c|c|c|}
\hline Method & $\epsilon_{y}$ & Trajectory rms \\
\hline 1-to-1 & $22.9 \pm 4.8 \epsilon_{y 0}$ & $72 \pm 1 \mu \mathrm{m}$ \\
\hline DF & $9.3 \pm 1.6 \epsilon_{y 0}$ & $55 \pm 1 \mu \mathrm{m}$ \\
\hline WF & $1.09 \pm 0.0 j$ & $44 \pm 1 \mu \mathrm{m}$ \\
\hline
\end{tabular}

To recapitulate, the correction algorithm is: (1) measure a difference orbit $\Delta x_{Q F}(s)$ created by varying the QFs and the associated dipole correctors, (2) measure the difference orbit $\Delta x_{Q D}(s)$ created by varying the QDs and the associated dipole correctors, (3) measure the actual trajectory $x(s)$, and finally, (4) one minimizes all three of these orbits. When developing the DF algorithm, it was found that a weighted least-squares is the best minimization procedure. Thus, in this variation, one minimizes the sum:

$$
\sum_{j \in\{B P M\}} \frac{\left(\Delta x_{Q F}\right)_{j}^{2}}{2 \sigma_{\text {prec }}^{2}}+\frac{\left(\Delta x_{Q D}\right)_{j}^{2}}{2 \sigma_{\text {prec }}^{2}}+\frac{x_{j}^{2}}{\sigma_{\mathrm{BPM}}^{2}+\sigma_{\text {prec }}^{2}},
$$

where each term is weighted by the accuracy of the respective measurement: $\sigma_{\mathrm{BPM}}$ is the estimated rms of the BPM misalignments and $\sigma_{\text {prec }}$ is the rms precision (reading-to-reading jitter) of the BPM measurements. Although it does not correct the wakefields due to the accelerator section misalignments, this technique will be referred to as Wake-Free (WF) correction because the corrected trajectory does not cause wakefield or dispersive dilutions.

\subsubsection{Simulations}

In Table 13, the performance of the DF and WF techniques is compared against the one-to-one algorithm in the $250 \mathrm{GeV} \mathrm{NLC} \mathrm{linac.} \mathrm{The} \mathrm{one-to-one} \mathrm{algorithm}$ adjusts the trajectory to zero the BPM measurements using only the BPMs and correctors located near the focusing quadrupoles. The results in Table 13 are the average of correcting 20 sets of random error distributions and the errors are the standard error on the results. In all cases, the trajectory is corrected at low intensity and then the intensity is increased to the clesign value.

The misalignment error distributions are the same as those used in the DF simulations described in Section 3.5.4, namely, $70 \mu \mathrm{m}$ rms vertical quadrupole and 
BPM misalignments, and $2 \mu \mathrm{m}$ rms BPM precision errors; the accelerator sections were aligned to the ideal machine centerline. In addition, the wakefields have been included and the optimal BNS energy spread has been added to the beam in all three cases. Finally, the initial conditions $\left(y_{0}, y_{0}^{\prime}\right)$ were optimized $^{[31]}$ after one-to-one correction to further reduce the dilution; while this procedure reduces the dilution from nearly $50 \epsilon_{y 0}$ when using the one-to-one algorithm, it yields little improvement when using DF or WF correction. The WF technique performs extremely well; it virtually eliminates all of the dispersive and wakefield emittance dilution and it does a better job correcting the actual trajectory than the other two methods.

Figures 36 and 37 illustrate the differences between the one-to-one correction technique and the WF method for one of the twenty cases used to calculate Table 13. Figure 36 compares the trajectory after. WF correction (upper plot) with the trajectory after one-to-one correction (lower plot). Likewise, Fig. 37 compares the emittance dilution after WF correction (upper plot) with the dilution after one-toone correction (lower plot). One can see that, after WF, the trajectory has a smaller rms and is "smoother" than after one-to-one correction and, more importantly, the emittance dilution after WF is much smaller than after one-to-one correction.

In the DF/WF techniques, the emittance dilution is "measured" by varying the quadrupole magnets. This measurement is then corrected to the level of the BPM precision. Thus, provided that the measurement resembles the emittance dilution, the dilution is constrained to the level of the BPM precision errors and does not increase along the length of the linac. This is illustrated in Fig. 37; the large spikes that occur in the emittance dilution arise because linac is corrected in sections and there is insufficient resolution at the beginning of each section to adequately correct the dilution.

Finally, Fig. 38 shows plots of the beam distribution after (a) one-to-one, (b) DF, and (c) WF correction for one of the twenty cases in Table 13. The scatterplots on the left are the projections of the beam distributions in the $y$ - $y^{\prime}$ phase space while the right-hand plots are projections onto the $y$ - $z$ plane; in both cases, we have represented the beam with 1000 macro-particles. One can immediately see that the beam emittance is seriously diluted after one-to-one correction; this is primarily due to dispersive errors. Next, after DF correction, the dispersive errors are corrected, but the distribution displays the tails characteristic of transverse wakefields; these arise from the random trajectory. Finally, after WF correction, one can see that the dilution due to both the dispersive errors and the wakefields is negligible. 


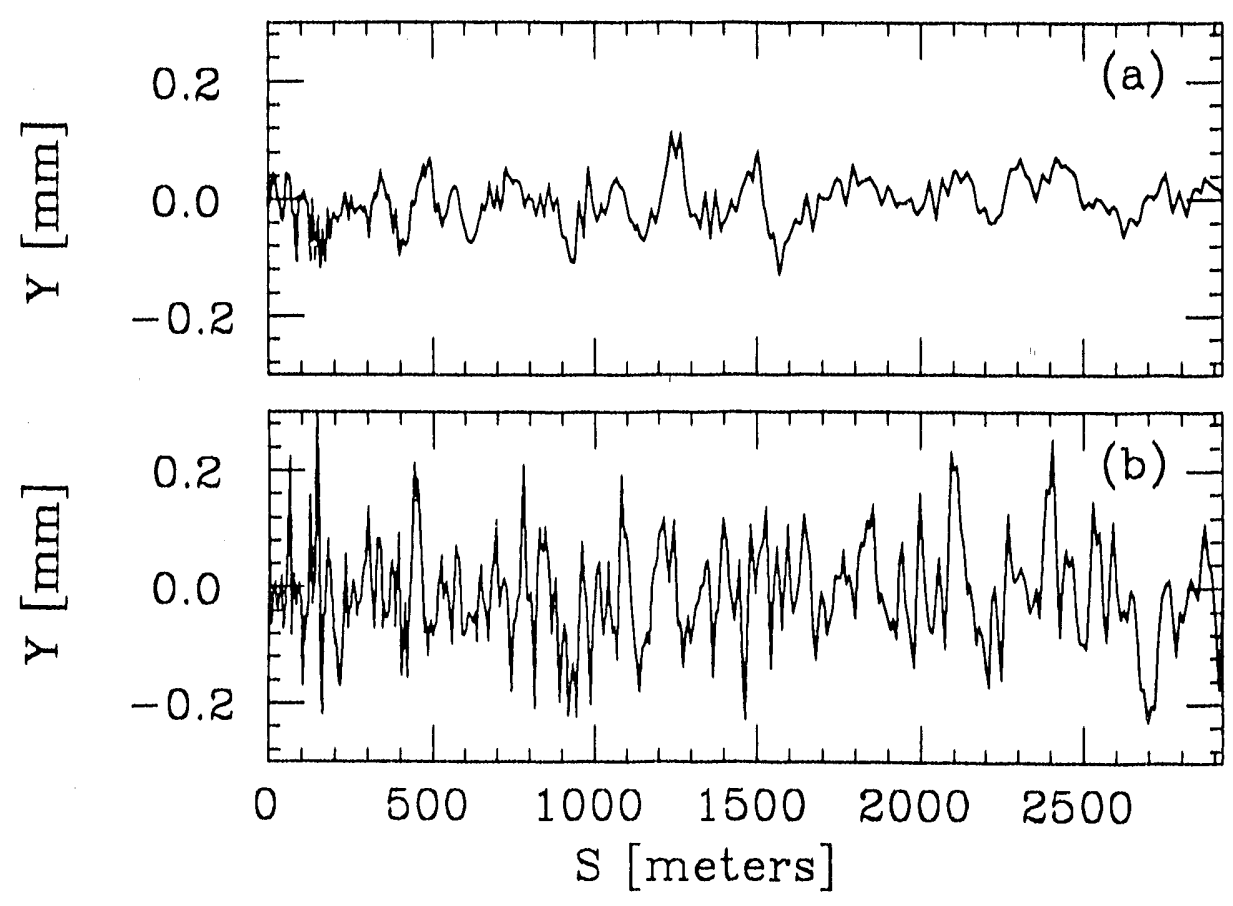

Fig. 36 The trajectory in the $250 \mathrm{GeV}$ NLC linac after (a) WF and (b) 1-to-1 correction.
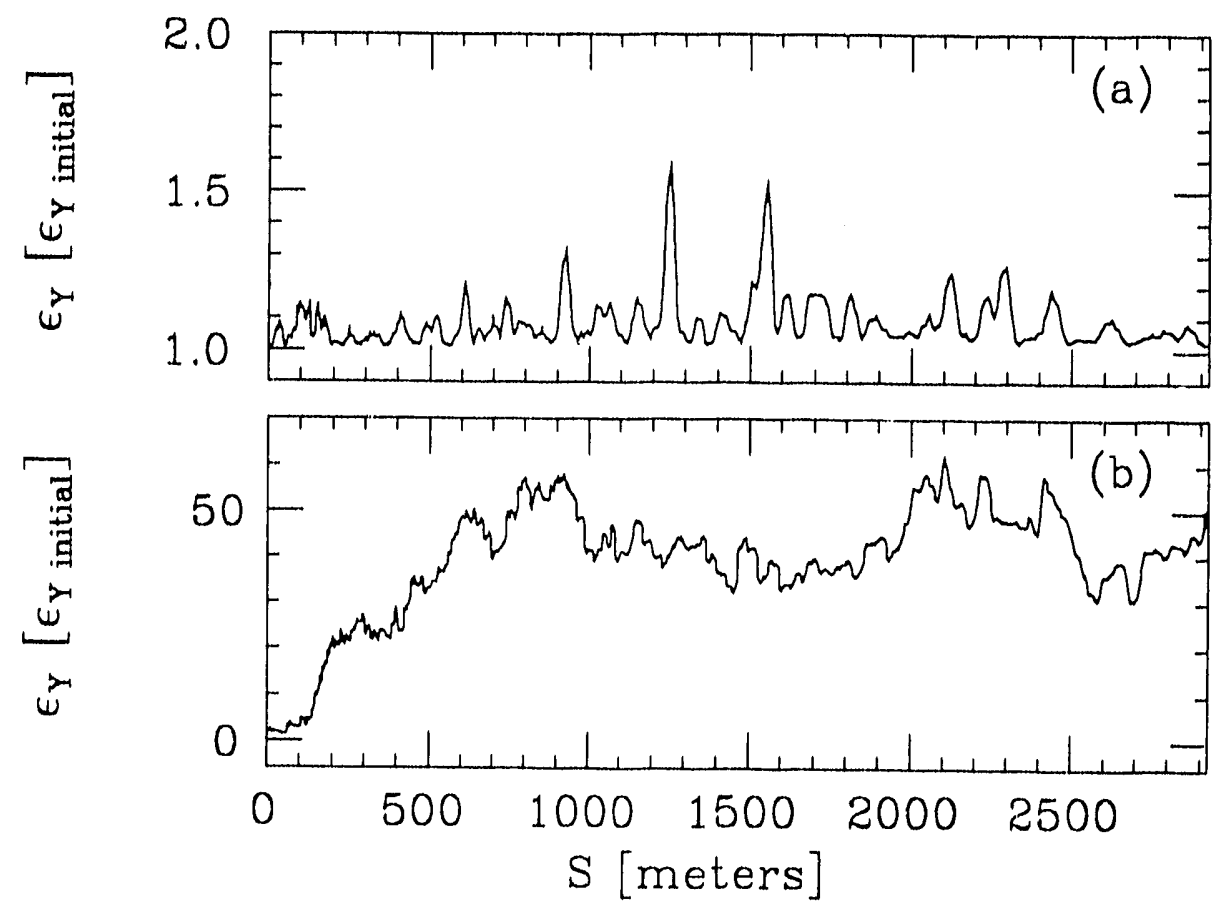

Fig. 37 The projected emittance dilution, in units of the injected emittance, in the $250 \mathrm{GeV}$ NLC linac after (a) W'F and (b) 1-to-1 correction. 


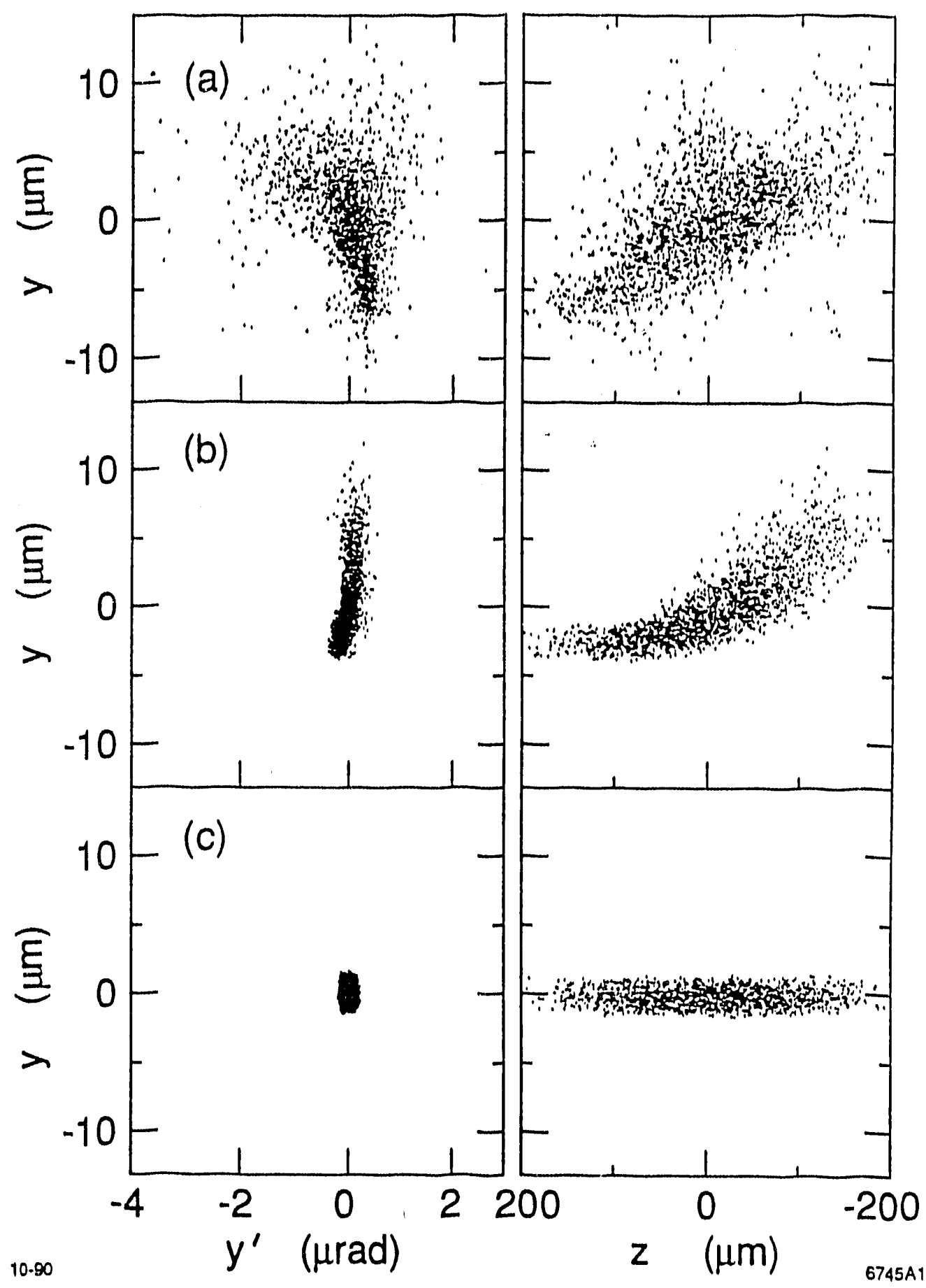

Fig. 38 The beam distribution after (a) 1-to-1, (b) DF, and (c) WF correction at the end of the $250 \mathrm{GeV} \mathrm{NLC} \mathrm{linac;} \mathrm{the} \mathrm{left-hand} \mathrm{plots} \mathrm{are} \mathrm{the}$ $y-y^{\prime}$ phase space while the right-hand plots are the beam in $y$ - $z$ space; from Ref. 87. 
Obviously, the WF technique reduces the emittance dilution due to misaligned BPMs, quadrupoles, and a non-zero trajectory extremely well. Since the technique is very similar to the DF method, we know that it is a robust algorithm and it is not sensitive to effects such as jitter and calibration errors.

\subsubsection{Further Extensions}

The WF correction technique does not correct the effect of misaligned accelerator sections. In fact, the WF method never actually measures the wakefields; it simply adjusts the trajectory such that any wakefield effects cancel. But, as noted in Eq. (3.5.23), the wakefields will shift the bunch centroid and thus it should be possible to measure the effects on the trajectory directly.

In theory, this could be performed by' measuring a difference orbit where the bunch length, bunch intensity, or beam energy and magnets are changed. Unfortunately, this becomes complicated because the beam loading changes the beam energy and energy spread, and thus, it is difficult to unravel the wakefield effects from the dispersive effects. Furthermore, the effect of the wakefields on the bunch centroid is much smaller than the effect on the beam tail where most of the emittance dilution occurs. Thus, it is difficult to make significant measurements.

We have performed some initial simulations of this technique ${ }^{[87]}$ more work is still needed to determine the feasibility of the approach.

\subsubsection{Tolerances with $\mathrm{DF} / \mathrm{WF}$}

The scaling of the emittance dilution with the misalignments can be found from the emittance dilution Eqs. (3.4.3) and (3.4.4). Furthermore, in Sections 3.4.1 and 3.4.2, we found that, when correcting the trajectory with the one-to-one technique, the dispersive and wakefield errors: $\Delta y_{d}$ and $\Delta y_{u}$, depend linearly upon the magnitude of the misalignments. Thus, the emittance dilution depends quadratically upon the magnitude of the misalignments when the dilution is small, less than $100 \%$, and the emittance dilution a linear function of the misalignment magnitude as the dilution increases.

In contrast, the dilution when correcting with the DF/WF techniques is ronghly independent of the misalignment magnitude. We have removed this dependence by scaling the trajectory measurements in Ec|s. (3.5.3) and (3.5.24) by the estimated rms of the misalignments. Thus, the cmittance dilution should only depend upon 


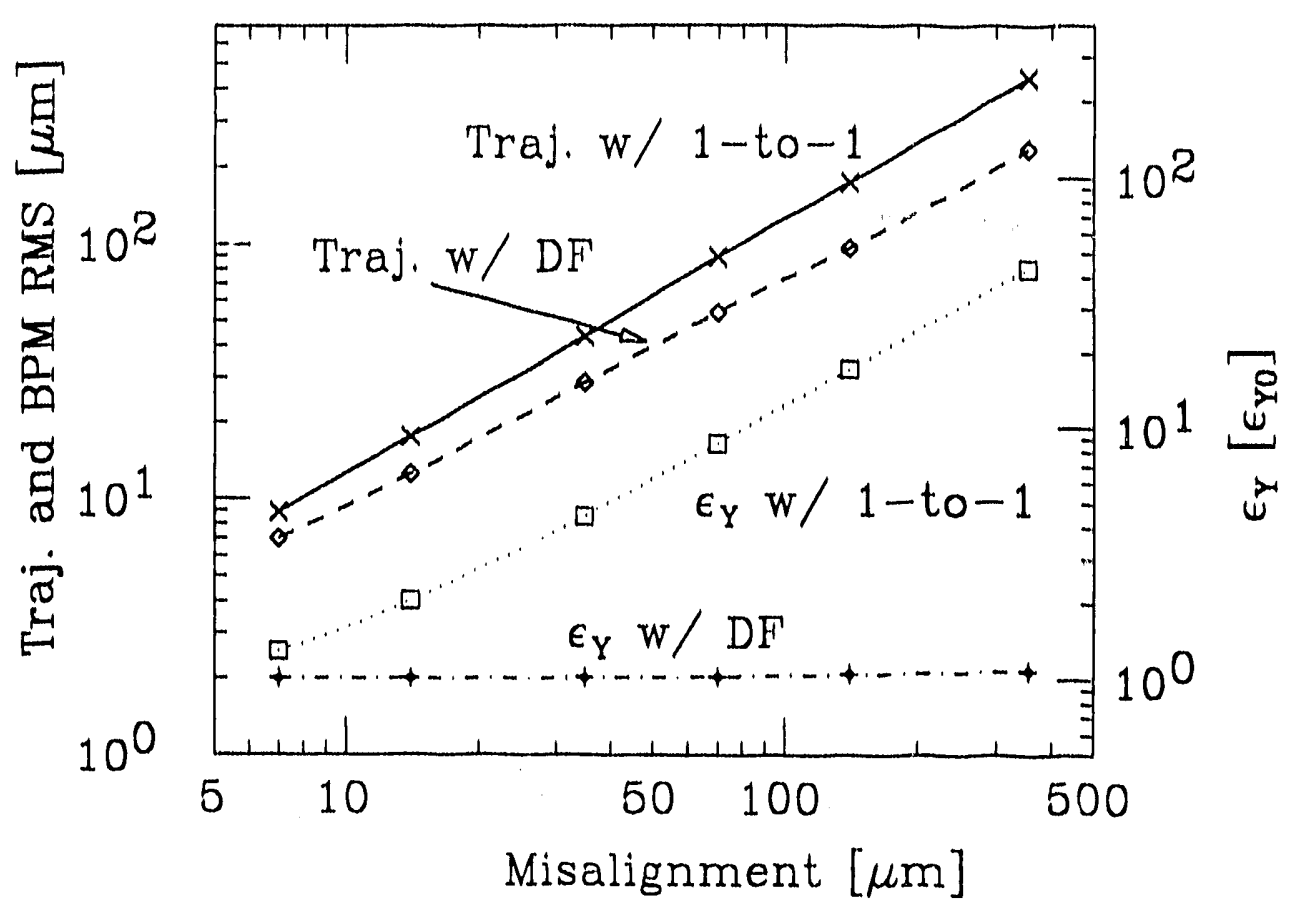

Fig. 39 One-to-one and DF correction versus the rms misalignment magnitude.

the other errors, namely, BPM precision, beam jitter, magnet scaling errors, and the RF deflections; these effects were discussed in Section 3.5.3.

The dependence of the trajectory correction techniques on the misalignment amplitude is illustrated in Fig. 39. Here, we have varied the rms magnitude of the vertical BPM and quadrupole misalignments from $7 \mu \mathrm{m}$ to $350 \mu \mathrm{m}$; note that all of the axes in Fig. 39 have logarithmic scales. The points plotted were found from the average of correcting twenty sets of random errors. The solid and dashed lines, at the top of the plot, are the rms values of the actual trajectory after correction with the one-to-one and DF techniques, respectively; these curves have scales on the left side of the figure. The DF technique is slightly better at correcting the actual trajectory, but the two curves are similar; in both cases the rms of the trajectory is roughly proportional to the rms of the misalignments.

The two other curves, the dotted and the dot-dash lines, are the emittances after correction with the one-to-one and DF techniques. The dilution after oneto-one correction is strongly dependent upon the misalignment magnitude. Here, the dilution varies from roughly $25 \%$ to over $3400 \%$ as the misalignments increase. In contrast, the dilution after DF correction is only weakly dependent upon the misalignment magnitude; it increases slowly from roughly $1 \%$ to $6 \%$ of the initial 
emittance as the misalignments become larger.

Thus, when using the DF/WI correction techniques, the emittance dilution is effectively uncoupled from the magnitucle of the transverse magnet misalignments. Instead, the DF/WF correction techniques impose relatively straight-forward toler" ances on the BPM precision, beam jitter, RF deflections, and magnet scaling.

\subsubsection{Discussion}

In this section, we have described two trajectory correction algorithms that can significantly reduce the emittance dilution due to a corrected trajectory. To fully determine the utility of these correction techniques, they need to be compared against the various alignment techniques. We will not perform such an analysis since it requires detailed knowledge of the sources of error and is machine specific. Instead, we will describe the difference between the DF/WF approach and that of the beam-based alignmert of Refs. 3 and 67 .

The DF/WF technique is similar to the beam-based alignment of Refs. 3 and 67 in that the alignment errors are detected by varying the effective beam energy, but, the approaches differ in the analysis of the data. The beam-based alignment technique uses the data to determine the individual BPM and quadrupole misalignments. Because the data has errors, these misalignments can only be determined with finite precision. Thus, large effective energy changes are used to increase the sensitivity to the individual misalignment errors. But, because the effective energy change is large, the beam-based technique is not especially sensitive to errors at the betatron frequency; this is the component that drives the wakefield and dispersive dilutions.

In contrast, the DF/WF technique finds a global solution to constrain the emittance dilution. Small effective energy changes are used so that the measured difference orbit resembles the actual emittance dilution. Thus, although the DF/WF is less sensitive to the individual misalignments, it is very sensitive to errors at the betatron frequency which cause the emittance dilution. This is illustrated in Fig. 40 where we have plotted the frequency spectrum of the trajectory after (a) WF correction and (b) one-to-one correction; the respective trajectory' rms's are $y_{c \mathrm{rms}}=51 \mu \mathrm{m}$ and $y_{c \mathrm{rms}}=94 \mu \mathrm{m}$. Notice that, although the WF technique only reduced the trajectory rms by a factor of two. the component at the betation frequency is reduced by roughly a factor of ten. Of course, using a smaller effective energy change may 

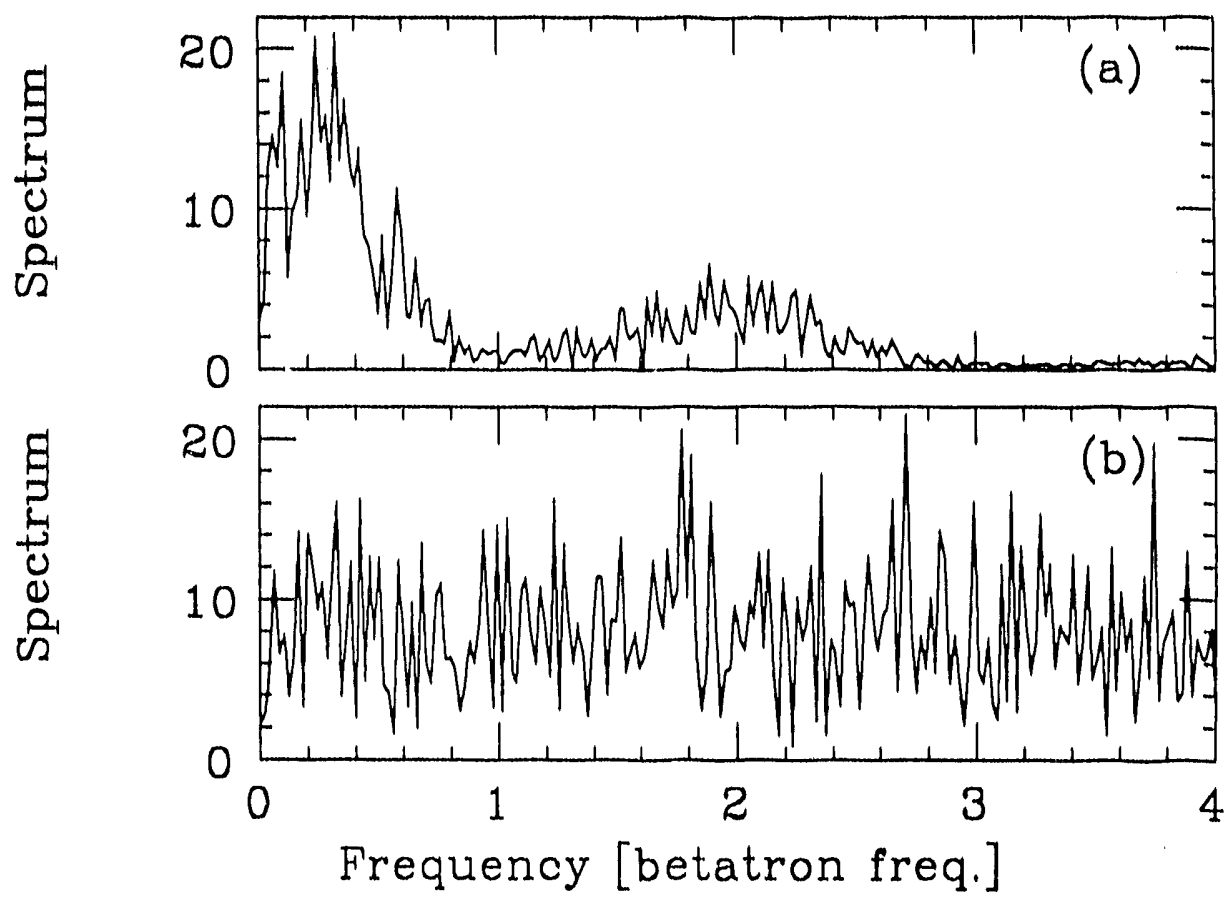

Fig. 40 The frequency spectrum of the trajectory after (a) WF and (b) one-to-one correction versus frequency in units of the betatron oscillation frequency; the trajectory $\mathrm{rms}$ 's are $y_{c \mathrm{rms}}=51 \mu \mathrm{m}$ and $y_{\mathrm{crms}}=94 \mu \mathrm{m}$ after WF and 1-to-1 correction, respectively.

make the DF/WF algorithm more sensitive to additional sources of error. Thus, the utility of the technique needs to be verified experimentally. Ultimately, it is likely to be found that the two techniques, the $\mathrm{DF} / \mathrm{WF}$ trajectory correction and the beam-based alignment, are complementary. 


\section{CHAPTER 3.6}

\section{ADDITIONAL CORRECTION METHODS}

In this section, we will discuss two additional techniques of correcting the emittance dilution due to transverse wakefields and RF deflections. Specifically, we will describe the use of RF deflecting cavities and then we will describe the use of trajectory bumps.

\subsubsection{RF Deflecting Cavities}

We can use an RF deflecting cavity to correct the emittance dilutions due to both the RF deflections from accelerator sections and the transverse wakefielcis; an $\mathrm{RF}$ deflecting cavity, like the transverse wakefield or an RF deflection, provides a transverse deflection that is a function of $z$, the longitudinal coordinate in the bunch. To crirect the dilutions, we use the $\mathrm{RF}$ cavities to remove any correlation between the transverse planes and $z$. This is simple, when correcting the RF deflections, since the time structure of the correcting fields can be adjusted to be similar to that of the RF deflections, but, it becomes more complicated when correcting the transverse wakefields; the wakefield defecting forces are highly nonlinear functions of $z$. Thus, to fully correct these dilutions, the deflecting cavity fields need to be shaped appropriately.

In the NLC, the wakefields appear to be a more serious limitation than the RF deflections, and thus, we will not discuss this technique further except to note that the correction cavities need to be distributed along the linac. The correction needs to be applied quasi-locally or the dilution will start to filament and thus becomes much harder to remove; this issue is illustrated when discussing the trajectory bumps in the next section.

\subsubsection{Trajectory Bumps}

The idea of using the beam trajectory to correct the wakeficld dilutions was first suggested in Ref. 31. There, the authors calculate the effect of inducing a coherent betatron oscillation to reduce the emittance dilution due to misalignments; the wakeficlds due to the betatron oscillation are used to cancel, on average, the wakefields due to the misalignments. 
Unfortunately, this approach does not work well in the NLC linacs since (1) BNS damping reduces the wakefields generated by the coherent oscillation and (2) the dilution partially filaments and thus needs to be corrected quasi-locally. The solution is to use multiple "non-dispersive" (ND) bumps to cancel the dilutions due to wakefields and RF deflections. In addition, dispersive bumps, i.e., closed betatron oscillations, can be used to cancel the dispersive emittance dilutions if desired.

The ND bumps can be created by deflecting the beam so that there are large offsets in the defocusing quadrupoles and smaller offsets in the focusing quadrupoles while the period of oscillation is equal to that of a betatron oscillation. In this way, the dispersive and BNS effects are cancelled while the wakefield deflections add. We can find an equation for the detailed trajectory using the equations for the dispersive error, Eqs. (3.4.1) and (3.4.2). Assuming that the energy spread is small so that we can neglect the chromatic phase advance, we have the condition

$$
0=\int_{0}^{s} d s^{\prime} \delta\left(G_{y}-K_{1} L_{4}\right) R_{12}\left(s^{\prime}, s\right)+\int_{0}^{s} d s^{\prime} \delta K_{1}^{\prime} y_{c} R_{12}\left(s^{\prime}, s\right)
$$

where $s$ is usually chosen to be equal to the betatron wave length.

An example of an ND bump in the $250 \mathrm{GeV}$ NLC linac is shown in Fig. 41. The bump consists of five oscillations so that it will have a strong effect on the wakefield dilutions. Although the bump is not perfectly closed, the dispersive emittance dilntion is less than $1 \%$ while the waliefield effects are large; they increase the emittance of an undiluted beam by a factor of 3.6. In Fig. 42, a single oscillation of this bump is compared with a betatron oscillation; the ND bump is the solid line while the betatron oscillation is plotted as a dashed line. Although, the period of oscillation is the same, the trajectories in the quadrupoles is quite different; the ND bump is created with dipole correctors at both the focusing and defocusing quadrupoles.

We have simulated the use of $N D$ bumps in reducing the emittance dilution due to misaligned accelerator sections in the $250 \mathrm{GeV}$ NLC linac. The averages of twenty simulations with $70 \mu \mathrm{m}$ rms accelerator section misalignments are listed in Table 14; the errors listed are the standard error. Without any correction, the emittance is increased by over a factor of 11 . Cptimizing the launch conditions $\left(y_{0}, y_{0}^{\prime}\right)$, to induce a coherent betatron oscillation, yields little improvement. As discussed, this occurs because the BNS damping reduces the wakefield dilution due to the oscillation but not the dispersive dilution. 


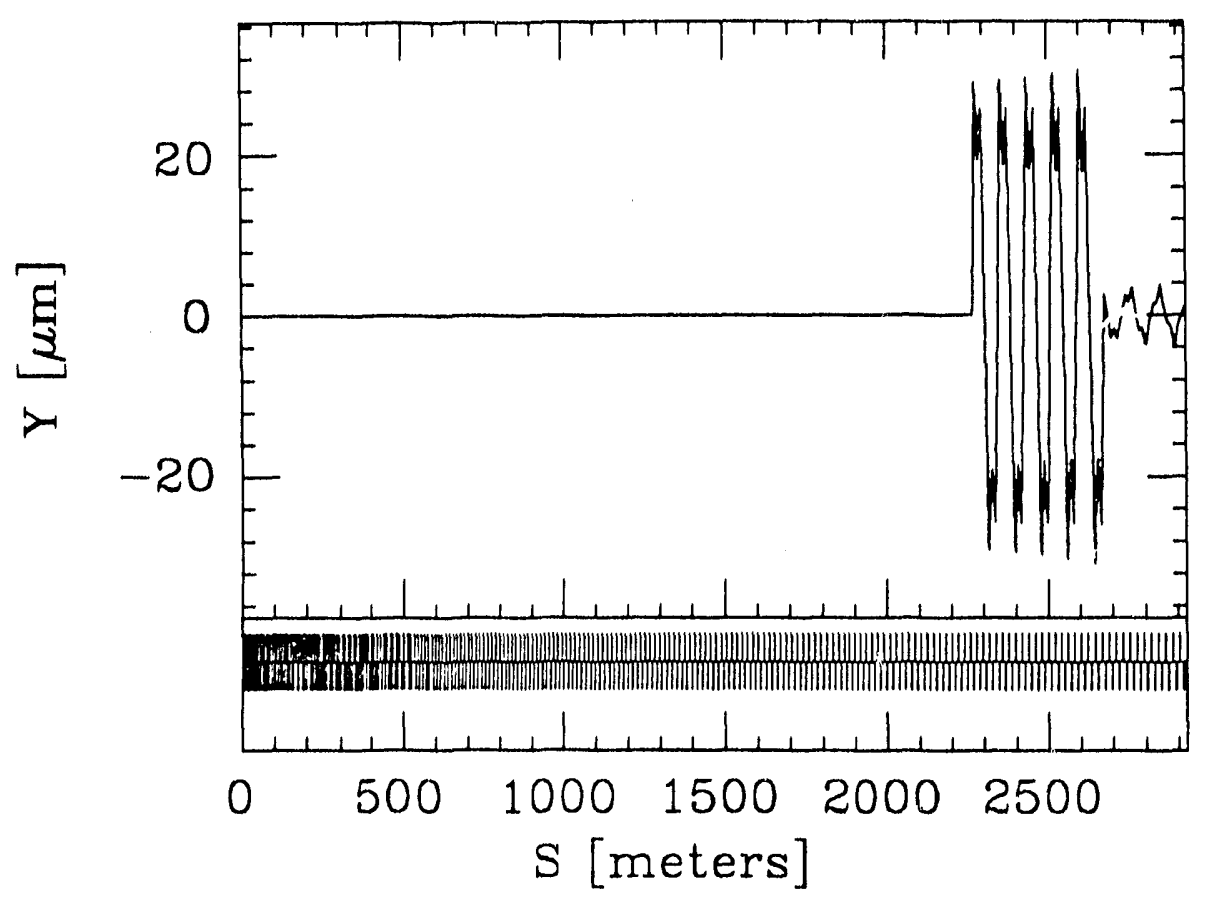

Fig. 41. Trajectory of a "non-dispersive" bump in the $250 \mathrm{GeV}$ NLC linac; the bump is slightly mismatched and does close perfectly. The lower window shows the locations of the quad."upoles.

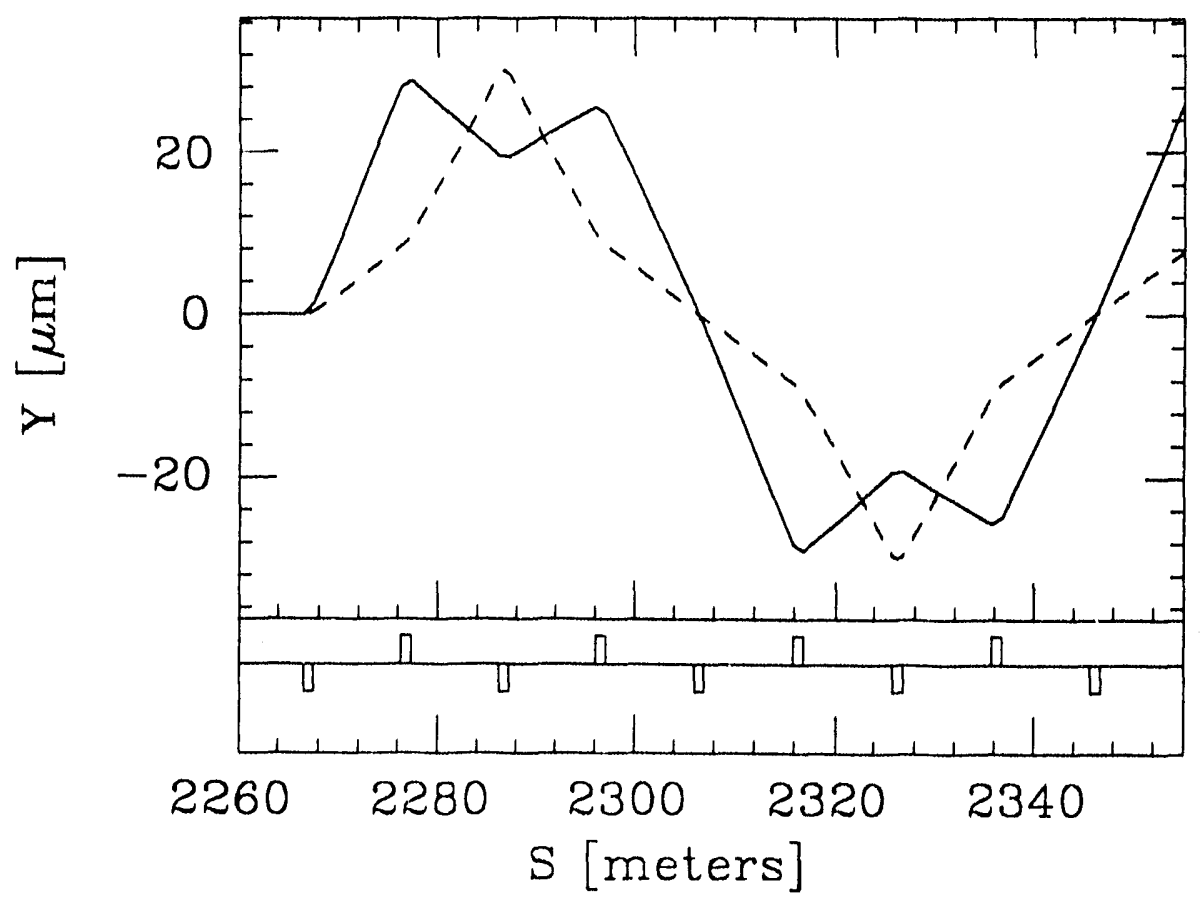

Fig. 42. Blow-up of Fig. 41 comparing the ND bump (solid line) with a betatron oscillation (dashed line); the vertically focusing magnets are plotted in the lower window as negative bars while the defocusing magnets are positive bars. 
Table 14. Simulations of ND bumps in the NLC linac.

\begin{tabular}{|c|c|}
\hline Uncorrected dilution & $11.7 \pm 2.1 \epsilon_{y 0}$ \\
\hline Initial conditions $\left(y_{0}, y_{0}^{\prime}\right)$ & $9.8 \pm 1.2 \epsilon_{y 0}$ \\
\hline $2 \mathrm{ND}$ bumps & $2.6 \pm 0.22 \epsilon_{y 0}$ \\
\hline $4 \mathrm{ND}$ bumps & $1.40 \pm 0.07 \epsilon_{y 0}$ \\
\hline $6 \mathrm{ND}$ bumps & $1.12 \pm 0.02 \epsilon_{y 0}$ \\
\hline
\end{tabular}

In contrast, the ND bumps are very effective at cancelling the dilution. Just two bumps, located near the beginning of the linac where the wakenelds are the most severe, reduces the emittance to $2.6 \epsilon_{y 0}$ from $11.7 \epsilon_{y 0}$; the two bumps are separated $90^{\circ}$ in phase to provide orthogonal controls. Of course, because of the filamentation, bumps near the front of the linac are not very effective at correcting the dilution originating at the end of the linac. Thus, we find improvement when we add another pair slightly further back, and even more improvement when we add a final pair at the end of the linac.

Finally, we need to discuss the algorithm used to optimize the emittance. In the simulations, we calculate the ND bump corrections by optimizing the emittance at the end of the linac with the bumps. This is a time intensive procedure, and, in a real linac, it would likely be impossible. Instead, one should break the linac into sections and use two orthogonal bumps to optimize each section. Although this requires multiple emittance measurements along the length of the linac, the final solution will be found much more quickly and accurately.

Thus, to summarize, these ND bumps can be used to effectively correct the wakefield dilutions; these dilutions may be due to misaligned accelerator sections or a corrected trajectory. Furthermore, the ND bumps can also be used to partially correct the dilutions due to RF deflections. Finally, although multiple bumps are needed to correct the dilutions before they begin to filament, these ND bumps would likely be easier (and cheaper) to implement than having independent movers on all of the accelerator sections as suggested in Ref. 106. 


\section{CHAPTER 3.7 MAGNETIC FIELD TOLERANCES}

In this section, we will calculate the effect of static magnetic field errors in the linacs. We assume that only the quadrupole magnets have field errors. These errors have three effects: (1) there are betatron mismatches due to the quadrupole errors, (2) there is transverse ccupling due to the skew quadrupole fields, and (3) there is rms emittance dilution due to strong nonlinear fields.

The equation for the vertical motion for generalized transverse magnetic fields can be written:

$$
y_{\beta}^{\prime \prime}-\sum_{n=1}^{\infty} K_{n} \operatorname{Im}(x+i y)^{n}+\sum_{n=1}^{\infty} \tilde{K}_{n} \operatorname{Re}(x+i y)^{n}=0
$$

where $K_{n}$ and $\widetilde{K}_{n}$ are the normal and skew field components analogous to $K_{1}$ and $\widetilde{K}_{1}$, and $x$ and $y$ include both the betation motion, $x_{\beta}$ and $y_{\beta}$, and the central trajectory. In the NLC, where the closed orbit is much greater than the betatron amplitude, the quadrupole field, found by expanding the higher order multipole fields about the central trajectory, usually has a more significant effect than the actual nonlinear fields; in essence, the bcams are so small that the fields always appear linear. This is one of the problems in attempting nonlinear collimation; ${ }^{[74}$ the nonlinear fields must be extremely large to have any nonlinear effect. For this reason, we will only consider the effect of the quadrupole and skew quadrupole field errors.

Finally, we should note that in making our estimates, we will neglect filamentation. Filamentation will make these effects worse and thereby force tighter tolerances. The reason for this is that once the beam filaments, the dilution is effectively not recoverable. Fortunately, the NLC linacs are in the partial filamentation regime and thus our estimates should be accurate. 


\subsubsection{Quadrupole Tolerances}

Quadrupole errors introduce beta mismatch errors. These are given by: ${ }^{[34]}$

$$
\frac{\Delta \beta(s)}{\beta(s)}=\int_{0}^{s} d s^{\prime} \Delta K^{\prime} \beta \sin 2\left(\psi(s)-\psi\left(s^{\prime}\right)\right)
$$

where $\Delta K$ is the quadrupole error: $\Delta K=\Delta K_{1}+2 K_{2} x_{c}+2 \widetilde{K}_{2} y_{c}+\cdots$. In addition, there are higher-order contributions, ${ }^{[123]}$ but these will be negligible in a linac where the design dispersion functions are zero.

\section{$\underline{\text { Random Frrors }}$}

Random quadrupole errors will cause a beta mismatch of:

$$
\left\langle\left(\frac{\Delta \beta(s)}{\beta}\right)^{2}\right\rangle=\int_{0}^{s} d s^{\prime}\left\langle\Delta K^{2}\right\rangle \beta^{2} \sin ^{2} 2\left(\psi(s)-\psi\left(s^{\prime}\right)\right)
$$

where in a FODO cell the minimum and maximum beta functions are given by Eq. (D.1.1). Now, the integral can be expressed as a sum over the FODO cells. In the NLC linac, $K_{1} L_{q} \beta$ is constant; the integrated quadrupole strength decreases as $\gamma^{-\frac{1}{2}}$ while the beta function increases with the square root of the energy. Furthermore, with this scaling, the phase advance per cell remains constant. Thus, the sum becomes

$$
\begin{aligned}
\left\langle\left(\frac{\Delta \beta(s)}{\beta}\right)^{2}\right\rangle=\left\langle\left(\frac{\Delta K}{K_{1}}\right)^{2}\right\rangle & \frac{4}{\cos ^{2} \psi_{c} / 2} \sum_{n=0}^{N_{\text {cell }}-1}\left[\left(1+\sin \psi_{c} / 2\right)^{2} \sin ^{2} 2\left(\psi(s)-n \psi_{c}\right)\right. \\
& \left.+\left(1-\sin \psi_{c} / 2\right)^{2} \sin ^{2} 2\left(\psi(s)-n \psi_{c}-\psi_{c} / 2\right)\right] .
\end{aligned}
$$

For reasonable phase advances, i.e, between $60^{\circ}$ and $120^{\circ}$ per cell, we can express this as

$$
\left\langle\left(\frac{\Delta \beta}{\beta}\right)^{2}\right\rangle \approx 4 \Lambda_{\text {cell }}\left\langle\left(\frac{\Delta K^{\prime}}{K_{1}}\right)^{2}\right\rangle\left(\frac{1+\sin \psi_{c} / 2}{\cos \psi_{c} / 2}\right)^{2}
$$

For the NLC main linac, a $1 \%$ tolerance on the quadrupole field yields an rms beta error of roughly $10 \%$; this is a relatively loose tolerance. 


\section{Systematic Errors}

The systematic quadrupole errors have a small effect unless the phase advance per cell approaches $180^{\circ}$. For cells with a phase advance of $90^{\circ}$, the systematic tolerance is roughly a factor of $\sqrt{N_{\text {cell }}}$ looser than the random tolerance and thus we will neglect it.

\subsubsection{Skew Quadrupole Tolerances}

In the case of skew quadrupole errors, we solve the equation for the vertical motion using the $R_{12}$ matrix element and the horizontal betatron motion. This yields

$$
\begin{aligned}
y_{\beta}(s)=- & \sqrt{\frac{\beta_{y}(s)}{\gamma(s)}} \sqrt{2 J_{x: 0} \gamma_{0}} \int_{0}^{s} d s^{\prime} \widetilde{K} \sqrt{\beta_{x} \beta_{y}} \\
& \times \sin \left(\psi_{x}\left(s^{\prime}\right)-\phi_{x 0}\right) \sin \left(\psi_{y}(s)-\psi_{y}^{\prime}\left(s^{\prime}\right)\right),
\end{aligned}
$$

where $\gamma_{0}$ is the initial relativistic factor, $J_{x 0}$ is the initial horizontal single particle invariant, $\phi_{x 0}$ is the initial horizontal phase, and $\widetilde{K}=\widetilde{K}_{1}-2 K_{2} y_{c}+\cdots$.

\section{$\underline{\text { Random Errors }}$}

To calculate the effect of random errors, we square Eq. (3.7.6) and calculate the expected skew quadrupole strengths. Thus, the double integral condenses to a single sum. Next, we perform an ensemble average over the beam particles, yielding

$$
\gamma(s) \frac{\left\langle y^{2}(s)\right\rangle}{\beta_{y}}=\gamma \epsilon_{x} \sum_{n=0}^{N_{q}-1}\left\langle\left(\widetilde{L^{\prime}} L_{q}\right)^{2}\right\rangle \beta_{x} \beta_{y} \sin ^{2}\left(\psi_{y}(s)-\psi_{y}\left(s_{n}\right)\right)
$$

At this point, we use the expression for the beta functions in a FODO lattice, Eq. (D.1.1); note that the horizontal and vertical beta functions are $180^{\circ}$ out of phase, when one is maximum the other is minimum. Then, we calculate the sum, finding

$$
\Delta \epsilon_{y} \approx 4 \epsilon_{x} N_{c e l l}^{\prime}\left\langle\left(\frac{\tilde{L^{\prime}}}{K_{1}^{\prime}}\right)^{2}\right\rangle
$$

Finally, if the skew quadrupole component is due to small rotations of the quadrupoles, where $\widetilde{K}=2 \Theta_{q} K_{1}$, we fincl a tolerance to limit the vertical emittance 
increase to $6 \%$ in the NLC main linac of

$$
\Theta_{q \mathrm{rms}} \lesssim 0.3 \mathrm{mrad} ;
$$

this yields a $3 \%$ luminosity reduction and is a fairly loose tolerance. Furthermore, notice that we have expressed the beam size increase in terms of an emittance increase. Actually, the coupling does not cause a real emittance increase; it increases the projected emittance. Thus, if the beam does not filament, the coupling can be corrected with four independent skew quadrupoles.

\section{Systematic Errors}

To estimate the effect of systematic errors, we start from Eq. (3.7.6) again, but now, we calculate the integral over the errors before squaring the expression. Assuming that the horizontal and vertical phase advances are similar and are not close to $180^{\circ}$, we find

$$
y_{\beta}(s)=-\sqrt{\frac{\beta_{y}(s)}{\gamma(s)}} \frac{\sqrt{2 J_{x 0} \gamma_{0}}}{2} \sum_{n=0}^{N_{q}-1} \tilde{\Lambda} \sqrt{\beta_{x} \beta_{y}} \cos \left(\psi_{x}\left(s_{n}\right)-\psi_{y}\left(s_{n}\right)+\phi_{x 0}+\psi_{y}(s)\right) .
$$

Now, we calculate the sum and average the square over the beam distribution. This yields an emittance increase

$$
\Delta \epsilon_{y} \approx \epsilon_{x} \frac{\left(\tilde{K}_{\mathrm{QF}}+\tilde{K}_{\mathrm{QD}}\right)^{2}}{\Pi_{1}^{2}} \frac{\sin ^{2} N_{\mathrm{cell}} \Delta \psi_{c} / 2}{\sin ^{2} \Delta \psi_{c} / 2}
$$

where $\Delta \psi_{c}=\psi_{x c}-\psi_{y c}$ and $\widetilde{K}_{\mathrm{QD}}$ and $\widetilde{K}_{\mathrm{QF}}$ are the skew quadrupole fields at the focusing and defocusing quadrupoles.

Notice that this expression depends upon the sum of the skew quadrupole fields at the focusing and the defocusing quadrupoles. Thus, if the quadrupoles have the same error, i.e., the same rotation or the same pole error, the emittance dilution will be small because the errors cancel. But, also notice that this expression goes as $N_{\text {cell }}^{2}$ when $\Delta \psi_{c} \lesssim 2 / N_{\text {cell }}$. Thus, it is desirable to separate the horizontal and vertical phase advances. In the NIC linacs, we are separating the phase advances by roughly six degrees per cell and thus this systematic tolerance is smaller than the random tolerance. 


\section{CHAPTER 3.8 PULSE-TO-PULSE JITTER}

In this section, we will calculate the pulse-to-pulse jitter which could be a severe limitation for future linear colliders since the vertical beam sizes are very small. This discussion will be similar to discussions found in Refs. 53, 97, 98, and 99. We have improved the accuracy of the estimates by using the correct scaling as is discussed in Appendix D; in general, this leads to tolerances that are less than a factor of $\sqrt{2}$ tighter. In addition, we have improved the estimate of the jitter due to ground motion finding that it is almost as severe as the effect of purely random jitter.

When discussing the jitter, we need to consider two regimes: rapid filamentation and no filamentation. In the case of rapid filamentation, the beam rapidly loses it's "memory" of the deflection and thus the kicks from different sources of jitter add linearly. In the other case, that without filamentation, the random sources of jitter cause the beam to perform a random walk and thus they add in quadrature, i.e., as the square root of the number of sources. The beam will only partially filament in the NLC linacs; and thus we neglect the filamentation when calculating tolerances.

In the case of weak filamentation, the jitter shifts the beams centroids and thus the colliding beams do not fully overlap at the IP. In the limit of small beam currents, the luminosity reduction is given by

$$
\mathcal{L}=e^{-\Delta^{2} / 4 \sigma^{2}} \mathcal{L}_{0}
$$

where $\Delta$ is the vertical offset between the two beams and $\mathcal{L}_{0}$ is the full luminosity. As the beam charge increases, the luminosity reduction decreases; this occurs because the two oppositely charged beams attract each other. ${ }^{[32,103]}$ For our purposes in the NLC, we will neglect the beam disruption and use Eq. (3.8.1) to estimate the reduction. Specifically, we will calculate tolerances to limit the jitter of each beam to less than $0.25 \sigma_{y}$; this corresponds to $3 \%$ luminosity reduction which is consistent with our other tolerances.

There are three primary sources of jitter: injection jitter, where the injected beam varies from pulse-to-pulse, trajectory changes due to movement of the quadrupole magnets, and trajectory changes due to power supply fluctuations. In addition, there are also effects that can arise from movement of the accelerator sections or variations of the RF power. 
We will start by estimating tolerances on the injection jitter. Then, we calculate the effect of random movement of the quadrupoles and the accelerator sections. This will lead to extremely tight tolerances. Fortunately, most sources of vibration lead to correlated motion of the magnets and accelerator sections and thus we discuss these effects next; unfortunately; these tolerances are similar. Finally, we calculate the effect of power supply fluctuations and then we discuss some of the feedback methods that may be used in the linac. Throughout, we only discuss the direct effect of the jitter, namely, the centroid shift.

\subsubsection{Injection Jitter}

If the beam does not filament, injection jitter directly causes position jitter at the IP. Since the jitter is demagnified along with the beam, the injection jitter tolerance, in units of the beam size, is the same as the IP jitter tolerance:

$$
y_{\mathrm{jit}} \lesssim 0.25 \sqrt{2} \sigma_{y}
$$

where, since we do not know the phase of the jitter relative to the IP, we included a factor of $\sqrt{2}$. This imposes stability tolerances on the damping rings and the bunch compressors. In the NLC main linac, we find a tolerance on the initial jitter of $y_{\mathrm{jit}} \lesssim 0.7 \mu \mathrm{m}$. Notice that this is the same as the tolerance due to the dispersive filamentation.

\subsubsection{Random Jitter}

\section{$\underline{\text { Magnets }}$}

Here, we will consider the effects of uncorrelated motion of the quadrupoles and the accelerator sections. Transverse movement of the quadrupoles will deflect the beam trajectory. Assuming that there is negligible filamentation, these deflections simply add:

$$
y_{c}=\sum_{n=0}^{N_{q}-1} K_{1} L_{q} y_{\mathrm{jit}} R_{12}\left(s_{n}, s_{f}\right)
$$

where $y_{\mathrm{jit}}(n)$ is the movement of the $n$th quadrupole, $L_{q}$ is the length of the magnet, and $N_{q}$ is the number of cuadrupole magnets. 
Assuming random jitter and using the NLC scaling, where the quadrupole strength decreases inversely with the beam energy while the length of the quadrupoles and the beta functions increase with the square root of the beam energy, we find

$$
\gamma_{f} \frac{\left\langle y_{c}^{2}\right\rangle}{\beta_{f}}=\frac{\left(\hat{\beta}_{0}+\dot{\beta}_{0}\right)}{4}\left(K_{1} L_{q}\right)_{0}^{2} \sqrt{\gamma_{0}} \sum_{n=0}^{N_{q}-1}\left\langle y_{j i t}^{2}\right\rangle \sqrt{\gamma_{n}}
$$

where the subscripts 0 and $f$ are used to denote the value at the beginning and end of the linac, and $\hat{\beta}_{0}$ and $\check{\beta}_{0}$ are the initial vertical beta functions at the focusing and defocusing quadrupoles.

Finally, using the relation between $\gamma$ and the quadrupole number, Eq. (D.1.7), we can evaluate the sum, finding

$$
\left\langle y_{c}^{2}\right\rangle \approx \frac{N_{y}\left\langle y_{\text {jit }}^{2}\right\rangle}{\cos ^{2} \psi_{c}^{\prime} / 2}
$$

where $\psi_{c}$ is the phase advance per cell and we have used Eq. (D.1.3) to simplify the result.

Using parameters for the NLC $500 \mathrm{GeV}$ linac, we find that a random magnet jitter of $5 \mathrm{~nm}$ leads to a trajectory jitter equal to one quarter the beam size; this is a tight tolerance. Of course, only a small portion of the magnet jitter is actually uncorrelated; most of the jitter is due to ground motion which we discuss next. But, turbulence in the niagnet cooling water or other local sources of noise can cause uncorrelated vibrations and thus these effects need to be examined in detail.

\section{Accelerator Sections}

If the jitter moves the ends of the accelerator sections so that the section is tilted, the beam will be deflected. We can estimate a tolerance following the procedure of the previous section. To limit the jitter to a quarter of the rms beam size, we find a tolerance of $250 \mathrm{~nm}$ on the random jitter of the ends of the sections, assuming a gradient of $100 \mathrm{MeV} / \mathrm{m}$ in the $500 \mathrm{GeV} \mathrm{NLC}$ linac; this is negligible. 


\subsubsection{Ground Motion}

As discussed in Section 2.4.1, the ground moves at the micron level at frequencies between roughly $0.1 \mathrm{~Hz}$ and $100 \mathrm{~Hz}^{|42|}$ the high frequency components of the motion are primarily due to cultural noise. Motion of the ground will cause correlated motion of the quadrupoles magnets which then deflect the beam trajectory. The effect of this ground motion has been estimated using the smooth approximation. ${ }^{\text {[8] }}$ Unfortunately, the smooth approximation can only estimate the lowest frequency resonance; it neglects the effect of the higher frequency resonances which are actually stronger. In general, resonances occur whenever ${ }^{(4,84)}$

$$
k_{\mathrm{gr}}=\psi_{c} / L_{c},\left(2 \pi \pm \psi_{c}\right) / L_{c},\left(4 \pi \pm \psi_{c}\right) / L_{c}, \cdots
$$

where $k_{\mathrm{gr}}$ is the wavenumber of the ground motion, $\psi_{c}$ is the phase advance per cell, and $L_{c}$ is the length of the cell.

We neglected the higher frequency resonances in the damping ring since they are far too high to be relevant; the power spectrum of the ground motion decays as $1 / f^{2}$. But, in the NLC linacs, the betatron wavelength varies from $20 \mathrm{~m}$ to roughly $120 \mathrm{~m}$. Assuming a phase velocity of $300 \mathrm{~m} / \mathrm{s}$, which is similar to the values measured at the SLAC and DESY sites, ${ }^{[1,95]}$ the lowest resonance occurs at frequencies above $2.5 \mathrm{~Hz}$ while the next two resonances occur at frequencies above roughly $8 \mathrm{~Hz}$ and $14 \mathrm{~Hz}$, assuming a $90^{\circ}$ phase advance per cell; these are still low enough to be important.

The motion of the quadrupoles in the three lowest frequency resonances is shown schematically in Fig. 43 where the lattice has a phase advance of $90^{\circ}$ per cell. Since the strengths of the focusing and defocusing quadrupoles are of opposite signs, it is apparent that the higher frequency lesonances have a greater effect than the lowest resonance; in the higher frecuency resonances the kicks from the focusing and defocusing quadrupoles add while in the lowest resonance the kicks subtract.

We will estimate the effect of the ground motion by considering the response to a plane wave traveling at an angle to the linac

$$
y_{\mathrm{gr}}(s)=y_{\mathrm{gr}} \cos \left(k_{\mathrm{gr}} s \cos \Phi+\phi\right)
$$

where $s$ is the distance along the accelerator, $\Phi$ is the angle between the ground wave and the linac, and $\phi$ is the ware phase. 


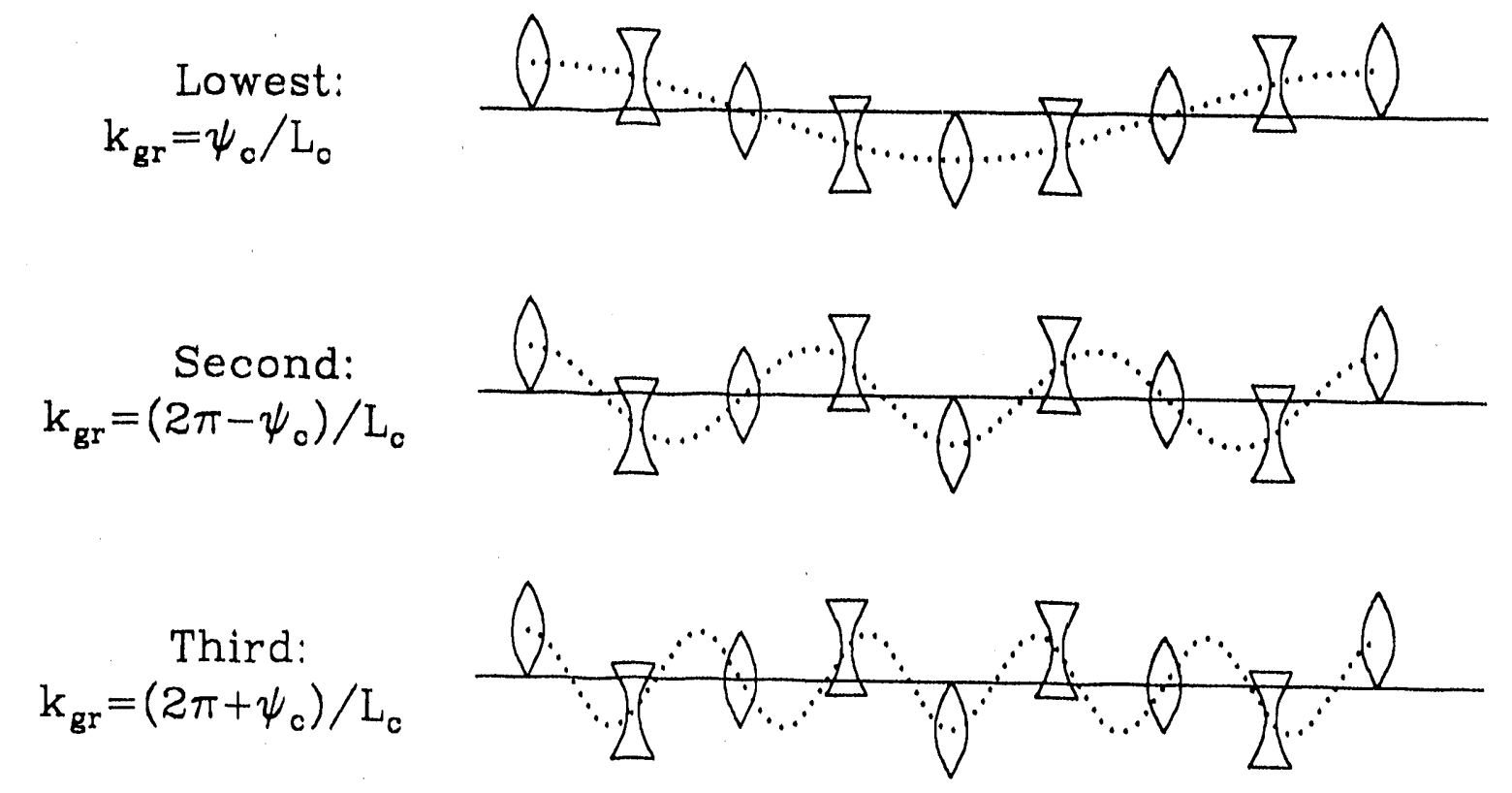

Fig. 43. The quadrupole motion in the three lowest frequency ground motion resonances where the phase advance per cell is $90^{\circ}$.

The response of the central trajectory is found from

$$
y_{c}(L)=\int_{0}^{L} d s K_{1}(s) y_{\mathrm{gr}}(s) R_{12}(s, L)
$$

Assuming the NLC scaling, where $K_{1}$ decreases inversely with the beam energy while $\beta$ and the length of the drifts and magnets increase with the square root the energy, this can be written

$$
\begin{array}{r}
y_{c}=\left(K_{1} L_{q}\right)_{0} y_{\mathrm{gr}} \sqrt{\frac{\gamma_{0}}{\gamma_{f}} \sqrt{\beta_{f}} \sum_{n=0}^{N_{q}-1}}\left(\frac{\gamma_{n}}{\gamma_{0}}\right)^{1 / 4} \cos \left(k_{\mathrm{gr}} s_{n} \cos \Phi+\phi\right) \\
\times \sqrt{\beta_{0}} \sin \left(\psi_{f}-n \psi_{c} / 2\right) \frac{K_{1}}{\left|K_{1}\right|},
\end{array}
$$

where $s_{n}$ and $\gamma_{n}$ are the position and energy at the $n$th quadrupole and $K_{1} /\left|K_{1}\right|$ changes sign between focusing and defocusing quadrupoles. Using simple trigono- 
metric relations, we can express the time averaged value of $y_{c}^{2}$ as

$$
\begin{aligned}
y_{c}^{2}= & \left(K_{1} L_{q}\right)_{0}^{2} \frac{y_{\mathrm{gr}}^{2}}{8} \frac{\gamma_{0}}{\gamma_{f}} \beta_{f} \mid \sum_{ \pm} \sum_{n=0}^{N_{\text {cell }}-1}\left(\frac{\gamma_{n}}{\gamma_{0}}\right)^{1 / 4} \\
& \times\left. e^{i\left(k_{\mathrm{gr}} \cos \Phi s_{n} \pm u \psi_{c}\right)}\left(\sqrt{\hat{\beta}_{0}}-\sqrt{\check{\beta}_{0}} e^{i\left(k_{\mathrm{gr}} \cos \Phi L_{n} / 2 \pm \psi_{\mathrm{c}} / 2\right)}\right)\right|^{2},
\end{aligned}
$$

where $\hat{\beta}_{0}$ and $\ddot{\beta}_{0}$ are the initial maximum and minimum beta functions, $N_{\text {cell }}$ is the number of cells, $L_{n}$ is the length of the $n$th cell, and the sum over \pm is a sum over both the sum phase and the difference phase.

In general, a sum of this form will exhibit resonances when $k_{\mathrm{gr}} \cos \Phi L_{c} \pm \psi_{c}=$ $2 \pi p$, where $p$ is an integer. In the NLC, the cell length scales with the square root of the beam energy and thus the resonance only occurs over a short distance. One can express the exact solution to this problem in terms of the Jacobi Theta functions; unfortunately, these are no simpler to evaluate. Thus, to evaluate the sum, we will use the method of stationary phase. In general, the method of stationary phase can be applied to an integral of the form ${ }^{[14]}$

$$
I=\int d s f(s) e^{i g(s)}
$$

where $f(s)$ is "slowly" varying and $|g(s)|$ is large. The method approximates the integral assuming that the only significant contribution comes when $g(s)$ is stationary and yields a result

$$
I \approx f\left(s_{0}\right) e^{i g\left(s_{0}\right)} \sqrt{\frac{i \pi}{2 g^{\prime \prime}\left(s_{0}\right)}}
$$

where $s_{0}$ is the point at which the first derivative of $g$ goes to zero and we have assumed that $g^{\prime \prime}\left(s_{0}\right) \neq 0$.

To apply this method in our case, we simply note that the phase is stationary whenever

$$
k_{\mathrm{gr}} L_{\mathrm{c}} \pm \psi_{\mathrm{c}}=2 \pi p \quad(p=0,1,2, \cdots)
$$

Now, using the NLC scaling, Eq. (D.1.7), and treating all of the resonances sepa- 
rately, we find a tolerance

$$
\begin{aligned}
\left\langle y_{c}^{2}\right\rangle \approx \frac{\pi N_{q} \tan \psi_{c} / 2}{2 L_{0}^{3}} \frac{\gamma_{0}}{\gamma_{f}}\left(\frac{y_{\mathrm{gr}}}{k_{\mathrm{gr}} \cos \Phi}\right)^{2} \\
\quad \times \sum_{\text {res }}\left(2 \pi p \pm \psi_{c}\right)\left(\sqrt{\hat{\beta}_{0}}-(-1)^{p} \sqrt{\dot{\beta}_{0}}\right)^{2}
\end{aligned}
$$

where $L_{0}$ is the initial cell length and the sum only includes the resonances encountered for a given wavelength.

Equation (3.8.14) shows that the long wavelength ground motion, which is resonant at the high energy end of the linac, is the most severe; the effect of any single resonance decays inversely with the frequency. There are two reasons for this: first, the adiabatic damping reduces the effect at the beginning of the linac, and second, when the resonance occurs at the end of the linac, the resonance condition includes more cells than when resonance occurs at the front of the linac.

At this point, we need to examine the scaling of our result. Eq. (3.8.14) explicitly depends inversely upon the cell length $L_{0}^{3}$, implying that longer cells greatly reduce the response to the ground motion. This is misleading. Using the relations

$$
N_{q} \propto \frac{\sqrt{\gamma_{f}}}{L_{0} \mathcal{G}} \quad, \quad k_{\mathrm{gr}} \propto \frac{1}{L_{0} \sqrt{\gamma_{0} \gamma_{f}}} \quad, \quad \beta_{0} \propto \frac{L_{0}}{\sin \psi_{c}}
$$

we find that the response scales as

$$
\left\langle y_{c}^{2}\right\rangle \propto \frac{y_{\mathrm{g} r}^{2}}{\cos ^{2} \psi_{c}^{\prime} / 2} \frac{\sqrt{\gamma_{f}}}{L_{0} \mathcal{G}} .
$$

Although, the effect of the ground motion is still eased by using longer cells and weaker focusing (larger beta functions), the dependance is seen to be much weaker. Furthermore, the effect depends inversely upon the accelerating gradient.

Now, we can use Eq. (3.8.14) to calculate tolerances. Assuming a worst case situation where the waves are parallel to the linac, i.e., $\cos \Phi=1$, the first resonance in the $500 \mathrm{GeV}$ NLC linac occurs at rouglily $2.5 \mathrm{~Hz}$. To limit the closed orbit jitter to $25 \%$ of the beam size, we find a tolerance on this component of the ground motion of $6 \mathrm{~nm}$. This is comparable to the effect of random jitter.

This tolerance will decay inversely with frequency until we encounter the next resonance at $8 \mathrm{~Hz}$. At this point, the first lattice resonance must be included and 


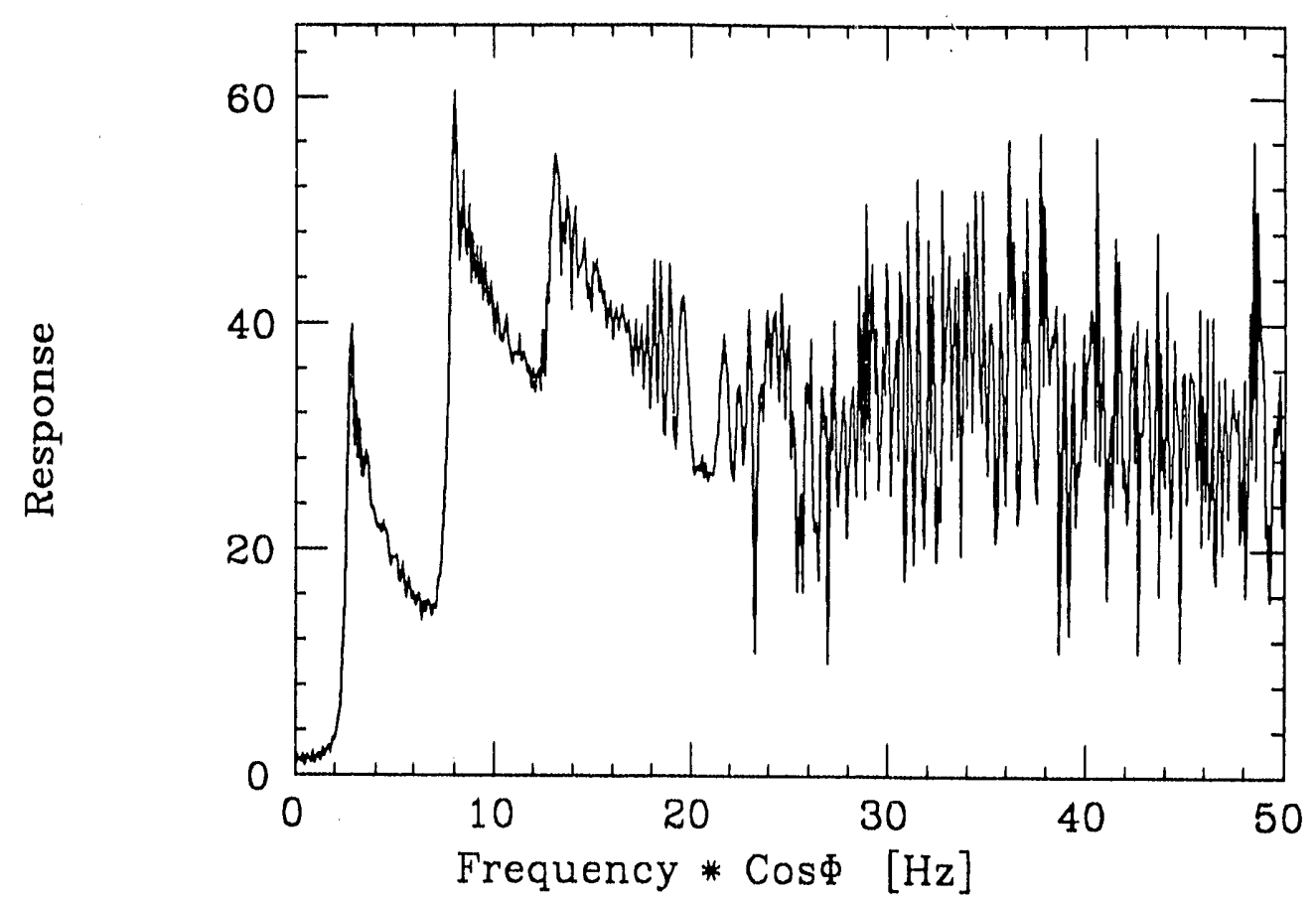

Fig. 44. Response function $R=\left|y_{\mathrm{jit}} / y_{\mathrm{gr}}\right|$ for ground motion in the $500 \mathrm{GeV}$ NLC linac.

we find a tolerance for jitter of $7 \mathrm{~nm}$ at $8 \mathrm{~Hz}$. Finally, this will also decay until we find the next resonance at roughly $14 \mathrm{~Hz}$. Here, we find a tolerance of $5 \mathrm{~nm}$ for jitter equal to $25 \%$ of the beam size. These tolerances are roughly a factor of twenty tighter than the estimates found in Refs. 97, 98, and 99.

To verify these results, we have simulated ground motion in the $500 \mathrm{GeV}$ NLC linac. The response of the trajectory to the ground motion is plotted in Fig. 44, where the response is defined as $R=\left|y_{c \mathrm{rms}} / y_{\mathrm{gr}}\right|$. Notice that, as predicted, there is an obvious resonance near $2 \mathrm{~Hz}$, another at $8 \mathrm{~Hz}$ and another at $14 \mathrm{~Hz}$. Above roughly $20 \mathrm{~Hz}$, many resonances interfere and the higher frequency resonances are not distinct. In the NLC linac, the final beam size is roughly $0.8 \mu \mathrm{m}$ and thus the tolerances found from Fig. 44 are very close to our estimates from Eq. (3.8.14).

To calculate a tolerance on the ground motion, we need to multiply the response function with the power spectrum for the ground motion. Instead, we simply note that the response is roughly 40 for frequencies above $8 \mathrm{~Hz}$. This implies a tolerance of $5 \mathrm{~nm}$ for all ground motion with frequency above $8 \mathrm{~Hz}$ and suggests that the ground motion will be a severe limitation in the future linear colliders.

Of course, we should note that our model for the magnet motion is rather simplistic: we have neglected the response of the magnet supports and we have 
treated the ground as an ideal material, without attenuation or inhomogeneities. In addition, we have assumed the worst case, where the ground motion is collinear with the linac. These effects should be included in future calculations; some will ease the tolerances and some will make them tighter.

Finally, there are a few methods of reducing the tolerances. One is to use feedback; we will discuss this in Section 3.8.5. In addition, one obviously wants to strive for a low noise site; most of the high frequency ground motion is cultural (man-made), and thus, it is highly site dependent. Furthermore, one wants to choose a site where the phase velocity of the ground waves is large; although this does not decrease the response, it shifts the resonances to higher frequencies where the amplitude of the ground motion is smaller. Lastly, if the ground motion has a distinct source, one can build the linacs perpendicular to the ground waves so that $\cos \Phi \approx 0$.

\subsubsection{Power Supply Fluctuations}

$\underline{\text { Magnets }}$

We will estimate three effects of power supply fluctuations: randcm variation of individual quadrupole power supplies, variation of a string of quadrupoles, assuming multiple quadrupoles per power supply, and finally, random variation of the dipole corrector power supplies. In all cases, we will only be concerned with the orbit offset caused by the fluctuation and we neglect the effect of the filamentation.

In the first case, the equation for the orbit offset is

$$
y_{c}=\sum_{n=0}^{N_{q}-1}\left(\frac{\Delta K_{1}^{\prime}}{K_{1}}\right) K_{1} L_{q} y_{\text {offset }} R_{12}\left(s_{n}, s_{f}\right)
$$

where $y_{\text {offset }}$ is the orbit offset in the quadrupole. This equation is virtually identical to Eq. (3.8.3). Thus, for the NLC scaling, we find

$$
\left\langle y_{c}^{2}\right\rangle \approx \frac{N_{q}}{\cos ^{2} \psi_{c} / 2}\left\langle\frac{\Delta K_{1}^{2}}{K_{1}^{2}} y_{\text {offset }}^{2}\right\rangle .
$$

In the $500 \mathrm{GeV} \mathrm{NLC} \mathrm{linac,} \mathrm{an} \mathrm{rms} \mathrm{power} \mathrm{supply} \mathrm{fluctuation} \mathrm{of} \Delta K_{1} / K_{1}^{\prime}=10^{-4}$ leads to an orbit jitter of one quarter the vertical beam size, assuming $50 \mu \mathrm{m}$ orbit offsets and individual power supplies for each quadrupole. 
This tolerance can be eased by connecting multiple quadrupoles to a power supply. In this case, we start from an equation similar to $\mathrm{Eq} \cdot(3.8 .17)$ except that $\Delta K_{1}$ does not vary between quadrupoles. If the trajectory were purely random, this tolerance would be identical to the previous one. But, as discussed in Section 2.2.4, a purely random trajectory in the quadrupoles will create a huge dispersion. Fortunately, the orbit correction that reduces the dispersion will also reduce the effect of the power supply fluctuations.

Finally, we need to consider the effect of fluctuations in the dipole corrector power supplies. As mentioned in Appendix D, there are two possible configurations for the dipole correctors: we could physically move the quadrupole magnets or we could use clectromagnets. Although, moving the magnets sounds more complex, there are disadvantages to the electromagnetic correctors; the power supply tolerances are one of them.

We will estimate a tolerance assuming that every quadrupole has an associated vertical dipole corrector which is irdividually powered. In this case, the tolerance is

$$
\left\langle y_{c}^{2}\right\rangle \approx \frac{N_{q}}{\cos ^{2} \psi_{c} / 2}\left\langle\frac{\Delta \theta^{2}}{\theta^{2}}\right\rangle \text {. }
$$

where we have assumed that the strength of the correctors decreases with the square root of the beam energy as does the integrated quadrupole strength.

In the $500 \mathrm{GeV} \mathrm{NLC} \mathrm{linac,} \mathrm{assuming} \mathrm{that} \mathrm{maximum} \mathrm{corrector} \mathrm{strength} \mathrm{must} \mathrm{be}$ at least $2 y_{\text {offset }} K_{1} L_{q}$, we find a tolerance of $\Delta \theta / \theta=5 \times 10^{-5}$ to limit the jitter to one quarter of the beam size.

\section{Accelerator Sections}

Unlike the magnet power supplies, it is much harder to regulate the $R F$ power to the structures. Since, as described in Section 3.4.3, the RF sections deflect the beam, RF power jitter can lead to vertical beam jitter.

The RF deflections can be expressed as in Eq. (3.4.35) and the effect of jitter in the $R F$ power can be written:

$$
\frac{\left\langle y^{2}\right\rangle}{\beta_{f}}=\frac{\overline{\beta_{f}}}{4} \sum_{n=0}^{N_{\mathrm{acc}-1}}\left\langle g^{2} \frac{\Delta V^{2}}{V^{2}}\right\rangle\left(\frac{\Delta \gamma_{\mathrm{RF}}}{\gamma_{n}}\right)^{2}\left(\frac{\gamma_{n}}{\gamma_{f}}\right)^{3 / 2}
$$

where $\Delta \gamma_{R F}$ is the energy gain per accelerator section, $g$ is the longitudinal-transverse coupling in the structure, and we have assumed the NLC scaling. At this 
point, we calculate the sum noting that $\gamma$ increases linearly with the number of accelerator sections. This yiclds a tolerance

$$
\left\langle y_{c}^{2}\right\rangle \approx g_{\mathrm{rms}}^{2}\left\langle\frac{\Delta V^{2}}{V^{2}}\right\rangle \frac{{\overline{\beta_{0}}}^{2} \Delta \gamma_{\mathrm{RF}}}{2 \gamma_{0}}
$$

where $\overline{\beta_{0}}$ is the initial average beta function in the accelerator sections; see Appendix D.

In the $500 \mathrm{GeV}$ NLC linac, we find a tolerance on the jitter of the accelerating voltage of $\Delta V / V \lesssim 3 \%$ to limit the jitter to one quarter of the beam size, assuming that $g_{\mathrm{rms}}=31 \mu \mathrm{rad}$ which is the tolerance determined in Section 3.4.3. This tolerance on the RF power jitter should be relatively easy to achieve.

\subsubsection{Feedback}

Obviously, these jitter tolerances are severe. One solution is to use feedback systems. Unfortunately, beam-based feedback in a linac is limited because the sampling rate, which is determined by the repetition rate, is usually low. In general, a broadband feedback system is only effective at frequencies much less than $f \lesssim f_{\text {rep }} / 6$. For example, numerous beam-based feedback systems have been implemented in the SLC. ${ }^{[55]}$ Here, the systems have been optimized to respond to a $\operatorname{tr}$ insient. A typical frequency and transient response are shown in Figs. 45 and 46 where the sampling rate is $60 \mathrm{~Hz}$. In this case, the crossover point, where the system response is $0 \mathrm{DB}$, occurs at $2 \mathrm{~Hz}$; this is at $f_{\text {rep }} / 30$.

If this is extrapolated to the NLC, with a repetition rate of $180 \mathrm{~Hz}$, we find a crossover point of $6 \mathrm{~Hz}$. This will not be sufficient to damp much of the ground motion. Fortunately, in any portion of the linac, the ground motion response is only resonant at a few narrow frequency bands. Thus, it may be possible to design narrow-band beam-based feedback systems that are effective at damping much higher frequencies than the broad-band systems; in theory, one should be able to damp frequencies approaching the Nyculust frequency with a narrow-band system.

Alternately, one can design an active clamping system for each of the components. Here, the sampling rate is dotermined by the component position detectors and not the beam repetition rate. Although, such systems are currently available, they tend to be expensive and complicated; developing systems suitable for a linear collider is a current topic of research. 
Chapter 3.9

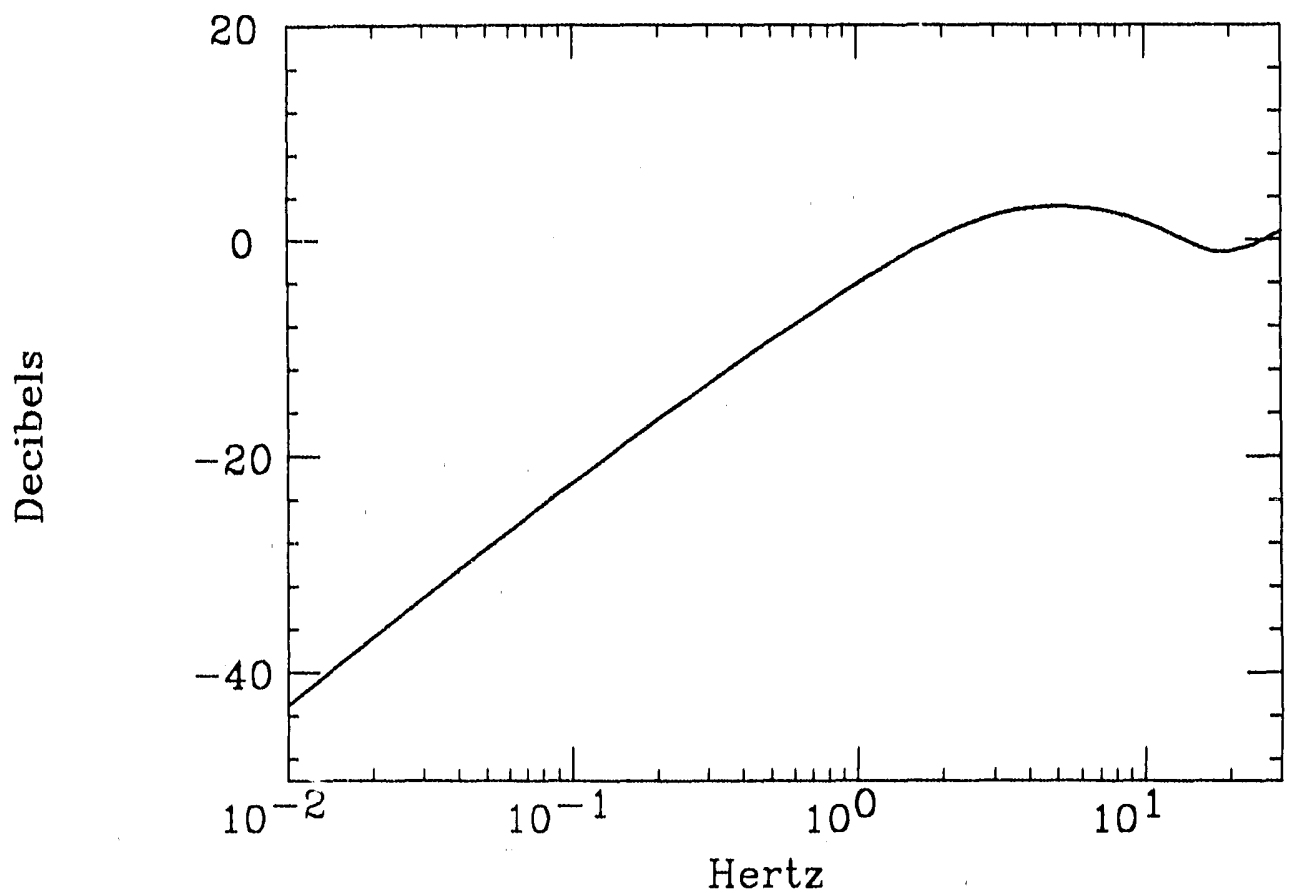

Fig. 45. Frequency response for SLC feedback system: $f_{\text {rep }}=60 \mathrm{~Hz}$; from Ref. 55.

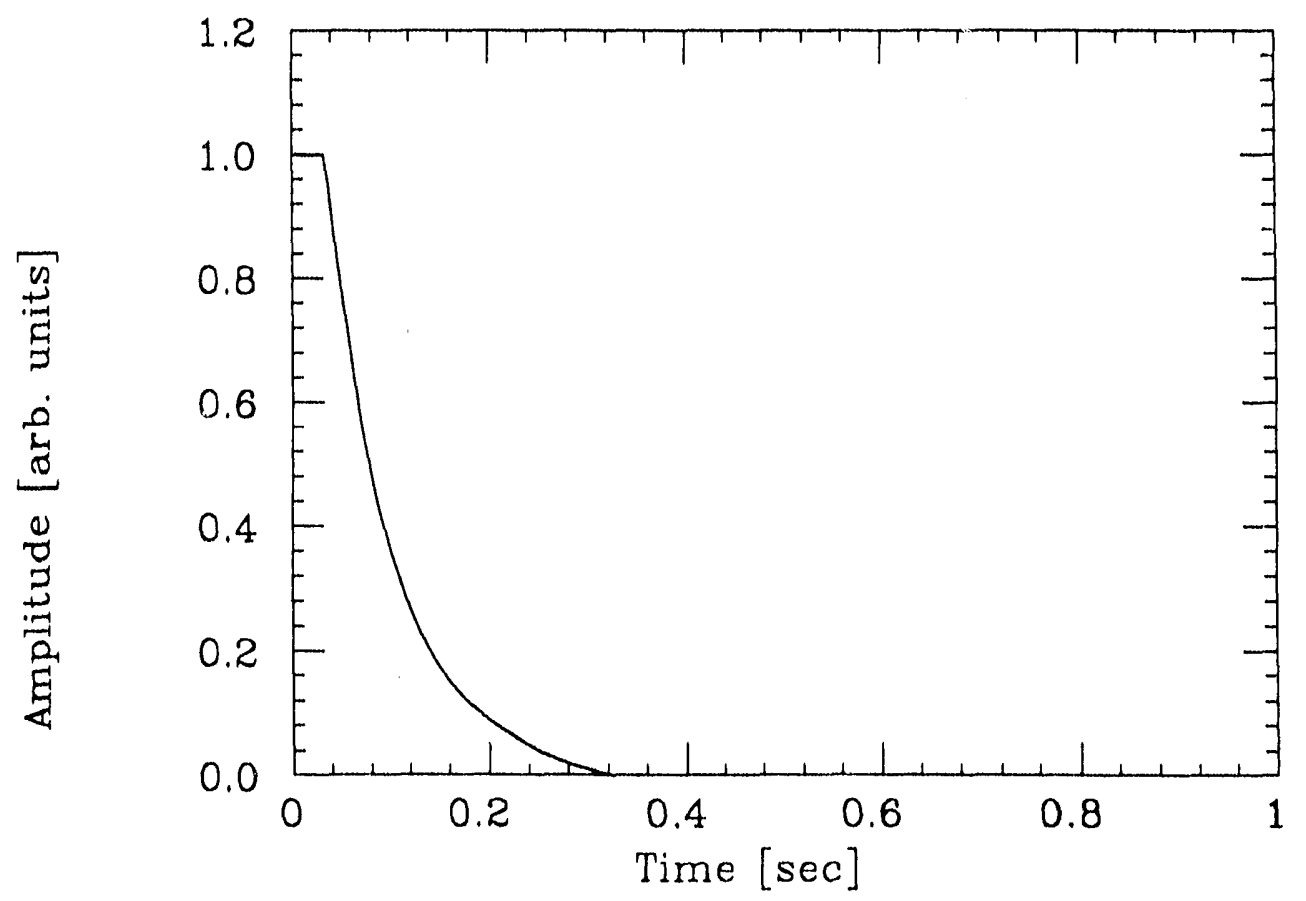

Fig. 46. Transient response for SLC feedback system: $f_{\text {rep }}=60 \mathrm{~Hz}$; from Ref. 55. 


\section{CHAPTER 3.9}

\section{ADDITIONAL EFFECTS}

In this chapter, we will discuss some additional sources of emittance dilution that have been neglected. Specifically, we will calculate the effect of the direct space charge field, synchrotron radiation, and scattering processes. In the NLC design, these all have negligible effect on the beam emittance.

\subsubsection{Space Charge}

As discussed in Section 2.3.3, the direct space charge field contains a factor of $1 / \gamma^{3}$ and thus the effects should decrease rapidly as the beam is accelerated. But, the space charge field also depends upon the bunch density. This is increased by the bunch compressions and by the adiabatic damping that occurs as the beam is accelerated. Thus, the effect of the space charge field actually only decreases as $1 / \gamma^{2}$ and because of the bunch compressions, the space charge field is roughly ten times more intense at the beginning of both the low and the high energy linacs than in the damping ring.

Fortunately, these forces are still weak compared to the external transverse focusing. Specifically, in the NLC damping ring, the space charge force caused a relative tune shift of $\Delta \nu_{y} / \nu_{y}=-0.001$. At the beginning of the low energy linac, the space charge phase shift is $\Delta \nu_{y} / \nu_{y}=-0.013$ and at the beginning of the high energy linac we find $\Delta \nu_{y} / \nu_{y}=-0.007$; this is comparable to the effect of the incoherent energy spread and should not pose a problem.

In Section 2.3.3, we also estimated the effect of the space charge driven betatron coupling resonances $2 \nu_{x} \pm 2 \nu_{y}$ when far from resonance. In a linear accelerator, the horizontal and vertical phase advances are usually similar. Thus, in this section, we will re-analyze the coupling assuming that we are near resonance. In the smooth approximation without acceleration, the equation for the vertical motion can be written

$$
\frac{d^{2} y}{d s^{2}}+k_{y}^{2} y=F_{y 01} y+F_{y 21} x^{2} y
$$

where $k_{y}=\overline{1 / \beta_{y}}$ and the coefficients $F_{y i j}$ are defined in Eq. (2.3.10). 
In the flat beam limit, where the horizontal motion is not perturbed, we can express Eq. (3.9.1) as

$$
\frac{d^{2} y}{d s^{2}}+\left[k_{y}^{2}-F_{y 01}-F_{y 21} J_{x} \beta_{x}-F_{y 21} J_{x} \beta_{x} \cos \left(2 s k_{x}+2 \theta_{x}\right)\right] y=0
$$

where $J_{x}$ is the single particle invariant, $\theta_{x}$ is the initial phase, and $k_{x}=\overline{1 / \beta_{x}}$. This is an example of Mathieu's equation, also referred to as a parametric oscillator, and it has a resonance when $k_{y}=k_{x}$, i.e., equal horizontal and vertical phase advances.

When close to the $k_{y}=k_{x}$ resonance, the solution to Mathieu's equation can be written $^{[14]}$

$$
y=a e^{s \kappa} \cos \left(s k_{x}+\theta_{1}\right)+b e^{-s \kappa} \cos \left(s k_{x}+\theta_{2}\right)
$$

where $a$ and $b$ are constants of the vertical motion, $\theta_{1}$ and $\theta_{2}$ depend upon $\kappa, \theta_{x}$, and the space charge force, and

$$
\kappa= \pm \frac{1}{4 k_{x}} \sqrt{\Delta^{2}-4\left(k_{y}^{\star 2}-k_{x}^{2}\right)^{2}}
$$

Here, $\Delta$ equals $F_{y 21} J_{x} \beta_{x}$ and $k_{y}^{\star 2}$ includes all of the constant terms with the square bracket of Eq. (3.9.2).

The vertical motion is unstable if $\kappa$ is real; one term grows exponentially. We can express this stability condition as

$$
\left|\frac{J_{x} \beta_{x}}{\sigma_{x}^{2}} \frac{\Delta \nu_{\mathrm{SC}}}{\nu}\right| \leq\left|\frac{4 \Delta \nu_{\mathrm{lat}}}{\nu}-\frac{4 \Delta \nu_{\mathrm{SC}}}{\nu}+\frac{2 \Delta \nu_{\mathrm{SC}}}{\nu} \frac{J_{x} \beta_{x}}{\sigma_{x}^{2}}\right|
$$

where $\Delta \nu_{\mathrm{SC}}$ is the shift in the phase advance due to the space charge and $\Delta \nu_{\text {lat }}=$ $\nu_{y}-\nu_{x}$ is the difference between the vertical and the horizontal phase advances. This stability condition implies that it is desirable to have the vertical phase advance greater than the horizontal by an arnount in excess of the space charge tune shift. By making the vertical phase advance greater than the horizontal, particles never encounter resonance, even as $J_{x}$ increases; this condition is also noted in Ref. 75 where the problem is solved including the perturbation to the horizontal plane.

Now, to determine the severity the coupling, we need to calculate the growth rate when on resonance. This is simply found from Eq. (3.9.4) and is given by 
$\kappa=\Delta / 4 k_{x}$ which is:

$$
\kappa=\frac{N r_{0}}{4 \sqrt{2 \pi} \sigma_{z} \gamma^{2}} \frac{1}{\sqrt{\gamma \epsilon_{x} \gamma \epsilon_{y}}} \frac{J_{x} \beta_{x}}{\sigma_{x}^{2}} .
$$

To find the expected growth, we need to integrate $\kappa$ over the length of the linac, i.e., we replace $e^{s \kappa}$ in Eq. (3.9.3) with $e^{\int d s \kappa}$. This integral can be written

$$
\int_{\gamma_{0}}^{\gamma_{f}} \frac{d \gamma}{\mathcal{G}} \kappa \quad \text { where } \quad \mathcal{G}=\frac{\gamma_{f}-\gamma_{0}}{L}
$$

and $L$ is the length of the linac.

In the NLC low energy linac, we find a maximum growth in amplitude of $30 \%$ for a particle with $J_{x} \beta_{x}=\sigma_{x}^{2}$; the inclease in $J_{y}$ of particles with larger amplitudes $J_{x}$ is greater. This is significant growth and indicates that we should stay far from the resonance. In the NLC linacs, a difference in phase advances of $6^{\circ}$ per $90^{\circ}$ cell should be sufficient to remove all growtli.

Finally, we note that since this coupling is a potential limitation, at least in higher current linacs such as the VLEPP design, the problem should be solved properly. This could be done by solving the Vlasov equation and examining the transient solution.

\subsubsection{Radiation}

Synchrotron radiation is enitted whenever the beam is deflected. Since the synchrotron radiation is a source of stochastic noise and can lead to emittance growth, the radiation could impose a tolerance on the trajectory offsets in the linear accelerator. In this section, we will calculate the vertical emittance dilution due to the opening angle of the radiation and the presence of vertical dispersion. In addition, we will calculate the increase in energy spread due to the radiation. In all cases, we will neglect the effect of the damping that is also due to the radiation; in general, this will be small provided that tla a radiation comprises a small percentage of the beam energy.

The effect of synchrotron radiation in a transport line is discussed in Refs. 69 and 105. We will use the results of Ref. 105 to calculate the emittance dilution due to the residual dispersion. In this case, the change in the vertical bean s'ze is given 
by

$$
\Delta \frac{\left\langle y_{\beta}^{2}\right\rangle}{\beta_{y}}=C_{2} \int_{0}^{L} d s E^{5}\left|G^{3}\right| \mathcal{H}_{y} \cos ^{2} \Phi
$$

where $C_{2}=4.13 \times 10^{-11} \mathrm{~m}^{2} / \mathrm{GeV}^{5}$ and the phase advance is $\Phi=\psi_{y}(L)-\psi_{y}(s)-$ $\tan ^{-1}\left(\alpha_{y}+\beta_{y} \eta_{y}^{\prime} / \eta_{y}\right)$. In our treatment, we will average over $\Phi$ assuming that the radiation is emitted uniformly. Thus, we find an emittance dilution

$$
\Delta \epsilon_{y}=\frac{C_{2}}{2} \int_{0}^{L} d s E^{5}\left|G^{3}\right| \mathcal{H}_{y}
$$

Now, we need to find expressions for $G$ and $E$ in the linac. We assume that the trajectory is randomly offset in every quadrupole. Furthermore, we assume that the dipole correctors are located at the quadrupoles. In this case, the angular deflection at each quadrupole is given by

$$
0=\left[\frac{\overrightarrow{r_{2}}-\overrightarrow{r_{1}}}{L_{\mathrm{drift}}}+\frac{\overrightarrow{r_{2}}-\overrightarrow{r_{3}}}{L_{\mathrm{drift}}}\right] \text {, }
$$

where $L_{\text {drift }}$ is the distance between quadrupoles and $\vec{r}_{i}$ is the radial position at three sequential quadrupoles. This yields an rms value for the inverse bending radius $G_{\mathrm{rms}}$ of

$$
G_{\mathrm{rms}}=\frac{2 \sqrt{x_{\mathrm{rms}}^{2}+y_{\mathrm{rms}}^{2}}}{L_{q} L_{\mathrm{drift}}}
$$

where $L_{q}$ is the length of the quadrupoles.

Next, we use the scaling for the lengths and the beta functions discussed in Appendix D; in the NLC linac they both scale with the square root of the beam energy. Thus, we can express the inlegral as a sum over quadrupoles

$$
\Delta \epsilon_{y}=8 C_{2} \sum_{n=1}^{\Lambda_{q}^{\prime}} \frac{L_{q 0}}{\overline{\beta_{0}}}\left\langle\eta_{y}^{2}\right\rangle \frac{E_{0}^{3} E^{2}\left(x_{\mathrm{rms}}^{2}+y_{\mathrm{rms}}^{2}\right)^{3 / 2}}{L_{q 0}^{3} L_{\mathrm{drift} 0}^{3}}
$$

where we have assumed that the dispersion is random and has a constant amplitude down the linac and the subscript o denotes values at the beginning of the linac. 
Finally, we use Eq. (D.1.7) to express the energy as a function of the quadrupole number and we assume $N_{q} \gg 1$ so that we can replace the sum with an integral over the quadrupole number. This yields

$$
\Delta \epsilon_{y}=\frac{8}{5} C_{2} N_{q} E_{0}^{3} E_{f}^{2} \frac{\left\langle\eta_{y}^{2}\right\rangle}{\overline{\beta_{0}}} \frac{\left(x_{\mathrm{rms}}^{2}+y_{\mathrm{rms}}^{2}\right)^{3 / 2}}{L_{q 0}^{2} L_{\mathrm{drift} 0}^{3}}
$$

In the $500 \mathrm{GeV} \mathrm{NLC} \mathrm{linac,} \mathrm{we} \mathrm{find} \mathrm{that} \mathrm{an} \mathrm{rms} \mathrm{vertical} \mathrm{dispersion} \mathrm{of} 3 \mathrm{~mm}$ with $100 \mu \mathrm{m}$ orbit offsets increases the vertical emittance by only $1 \%$. This is a loose tolerance; we need to keep the vertical clispersion below a few hundred microns for other reasons. Furthermore, the relative emittance dilution only increases as $E_{f}^{3 / 2}$ and thus it should not become a significant limitation even at a much higher final energy.

Next, we can estimate the emittance contribution from the opening angle. Using the results of Section 2.2.3, we can express the emittance dilution as

$$
\Delta \epsilon_{y}=\frac{13 C_{2}}{220} \int_{0}^{L} d s\left|G^{3}\right| E^{5} \frac{\overline{\beta_{0}}}{\gamma^{2}} .
$$

Following the procedure used to calculate the dispersive dilution and using the NLC scaling, we find

$$
\Delta \epsilon_{y}=\frac{26}{165} C_{2} N_{4} \overline{\beta_{0}} \frac{E_{0}^{4} E_{f}}{\gamma_{0}^{2}} \frac{\left(x_{\mathrm{rmss}}^{2}+y_{\mathrm{rms}}^{2}\right)^{3 / 2}}{L_{40}^{2} L_{\mathrm{drift} 0}^{3}}
$$

In the $500 \mathrm{GeV} \mathrm{NLC} \mathrm{linac,} \mathrm{the} \mathrm{opening} \mathrm{angle} \mathrm{contributes} \mathrm{a} \mathrm{relative} \mathrm{emittance} \mathrm{dilu-}$ tion of $10^{-6}$ for $100 \mu \mathrm{m}$ orbit offsets; this is a negligible effect.

Finally, we can calculate the expected increase in the energy spread:

$$
\Delta \sigma_{\epsilon}^{2}=\frac{C_{2}}{E_{f}^{2}} \int_{0}^{L} d s\left|G^{3}\right| E^{7} .
$$

With the NLC parameters, we find

$$
\Delta \sigma_{\epsilon}^{2}=\frac{4}{5} C_{2} N_{q} E_{0}^{\frac{3}{2}} E_{f}^{\frac{5}{2}} \frac{\left(x_{\mathrm{rms}}^{2}+y_{\mathrm{rms}}^{2}\right)^{3 / 2}}{L_{\eta 0}^{2} L_{\mathrm{drift} 0}^{3}} .
$$

In the $500 \mathrm{GeV} \mathrm{NLC} \mathrm{linac,} \mathrm{this} \mathrm{predicts} \mathrm{an} \mathrm{increase} \mathrm{in} \mathrm{the} \mathrm{relative} \mathrm{energy} \mathrm{spread} \mathrm{of}$ $10^{-5}$ which, again, is negligible. 


\subsubsection{Scattering}

So far we have neglected all of the scattering effects such as intrabeam and Touschek scattering, ion trapping, and beam-gas scattering. These phenomena are discussed in reference to the damping, ring in Sections 2.3.1, 2.3.2, and 2.3.6. In general, the cross sections of these effects are too small to be relevant for emittance dilution in a linac and thus it is valid to ignore them.

As an example, we will estimate the transverse emittance growth due to elastic beam-gas scattering. We start from Eq. (2.3.36) to calculate the rate the rms angle is increased by the scattering. This yields

$$
\Delta \gamma \epsilon_{y}=\int_{0}^{L} d s 4 \pi n_{\mathrm{gas}} Z(Z+1) r_{0}^{2} \frac{\beta_{y}}{\gamma}\left[\ln \left(\frac{\theta_{\max }}{\theta_{\min }}\right)-\frac{1}{2}\right] .
$$

Strictly, $\theta_{\max }$ and $\theta_{\min }$ are functions of $\gamma$, but the dependence is weak and thus we simply estimate the factor within the square brackets as 20 . Furthermore, both $\beta$ and $\gamma$ are functions of $s$. In the NLC linac, the beta function grows with the square root of the beam energy and thus the integrand decreases as $\gamma^{-\frac{1}{2}}$. We assume that $\gamma_{f} \gg \gamma_{0}$ and find

$$
\Delta \gamma \epsilon_{y}=160 \pi \operatorname{Ln}_{\mathrm{gas}} Z(Z+1) r_{0}^{2} \frac{\overline{\beta_{0}}}{\sqrt{\gamma_{0} \gamma_{f}}} .
$$

Assuming 5000 meters of linac starting at a an energy of $2 \mathrm{GeV}$ and having a residual gas of carbon monoxide with a pressure of $10^{-7}$ Torr, we find an emittance dilution of roughly 1\%. Furthermore, we need to remember that it is dominated by the large angle scattering events; see the discussion in Section 2.3.6. This may have implications for the collimation that will be needed, but it does not represent a dilution of the core emittance. 


\section{CHAPTER 3.10 ACCELERATION SUMMARY}

In this chapter, we have discussed and evaluated most of the effects that can dilute the vertical emittance during acceleration in a linac. The primary sources of dilution are conservative dilutions and pulse-to-pulse beam jitter. The conservative dilutions increase the projected vertical enittance by coupling the vertical phase space to the longitudinal and horizontal emittances. This coupling does not an tually increase the beam emittance; in theory, it is always correctable. Unfortunately, in a linac the dilution filaments, becoming extremely difficult to correct and effectively equivalent to an emittance increase.

These conservative dilutions impose tolerances on the magnitude of coherent betatron oscillations and the transverse alignment of the magnets, BPMs, and accelerating structures. We have derived analytic expressions to estimate the tolerances, assuming that the linac is composed of FODO focusing cells that are scaled with the square root of the beam energy and neglecting the filamentation; this is valid for designs in the partial filamentation regime but will under-estimate the tolerances for machines with strong filamentation. These derived expressions were used to calculate tolerances for the NLC linacs and they were confirmed with simulations.

Tolerances for the $500 \mathrm{GeV}$ NLC main linac are listed in Table 15. The tolerance on a coherent oscillation specifies a tolerance on the BPM precision; currently, BPMs with sub-micron precision are being developed. In addition, the dispersive dilutions and wakefields set the alignment tolerances; without any form of special correction, these are only a few microns. The beam-based alignment techniques may be able to achieve such tolerances. Alternately, the emittance dilutions can be corrected, provided that the correction is performed before the dilution filaments. We have described two styles of correction: DF/WF trajectory correction and non-local bumps. One can see, in Table 15, that these techniques can substantially ease the required alignment tolerances. Of course, these tolerances are not final; detailed simulations need to be performed including all of the error effects. But, the implication is that, in a machine with weak filamentation, we can use correction techniques to ease the aligninent tolerances by roughly one order of magnitude.

The other important source of dilution is pulse-to-pulse jitter, which affects both the beam centroid and beam size. We have estimated the centroid jitter, again neglecting the filamentation; the beam size jitter is primarily due to filamentation 
Table 15. Summary of conscrvative dilutions and tolerances in the $500 \mathrm{GeV}$ NLC main linac.

\begin{tabular}{|c|c|c|c|}
\hline Source & Tolerance & Dilution & $\Delta \epsilon_{y}$ \\
\hline $\begin{array}{l}\text { Injected betatron oscillation } \\
\text { with BNS damping }\end{array}$ & $y_{0} \lesssim 0.7 \mu \mathrm{m}$ & $\begin{array}{l}\text { Dispersive } \\
\text { Wakefield }\end{array}$ & $\begin{array}{l}6 \% \\
0 \%\end{array}$ \\
\hline $\begin{array}{l}\text { BPM and quad. misalignments } \\
\text { with 1-to-1 traj. correction }\end{array}$ & $y_{m} \lesssim 4 \mu \mathrm{m}$ & $\begin{array}{l}\text { Dispersive } \\
\text { Wakefield }\end{array}$ & $\begin{array}{l}6 \% \\
3 \%\end{array}$ \\
\hline $\begin{array}{l}\text { BPM and quad. misalignments } \\
\text { with special correction }\end{array}$ & $y_{m} \lesssim 50 \mu \mathrm{m}$ & $\begin{array}{l}\text { Dispersive } \\
\text { Wakefield }\end{array}$ & $\begin{array}{l}10 \% \\
10 \%\end{array}$ \\
\hline $\begin{array}{l}\text { Acc. section misalignments } \\
\text { without correction }\end{array}$ & $y_{m} \lesssim 4 \mu \mathrm{m}$ & $\begin{array}{l}\text { Wakefield } \\
\text { RF Deflect. }\end{array}$ & $\begin{array}{l}6 \% \\
0 \%\end{array}$ \\
\hline $\begin{array}{l}\text { Acc. section misalignments } \\
\text { with ND bumps }\end{array}$ & $y_{m} \lesssim 50 \mu \mathrm{m}$ & $\begin{array}{l}\text { Wakefield } \\
\text { RF Deflect. }\end{array}$ & $\begin{array}{l}10 \% \\
4 \%\end{array}$ \\
\hline Quadrupole rotations & $\Theta \lesssim 0.3 \mathrm{mrad}$ & $\beta$ coupling & $6 \%$ \\
\hline
\end{tabular}

Table 16. Summary of jitter tolerances in the $500 \mathrm{GeV}$ NLC main linac.

\begin{tabular}{|c|c|c|}
\hline Source & Tolerance & $\Delta \mathcal{L}$ \\
\hline Uncorrelated quad. movement & $y_{\text {jit }} \lesssim 5 \mathrm{~nm}$ & $-3 \%$ \\
\hline $\begin{array}{c}\text { Correlated quad. movement at } \\
\text { frequencies } f \gtrsim \mathrm{Hz}\end{array}$ & $\begin{array}{c}y_{\text {jit }} \lesssim 5 \mathrm{~nm} \\
\text { (worst case) }\end{array}$ & $-3 \%$ \\
\hline Quadrupole power supply jitter & $\Delta K_{1} / K_{1} \lesssim 10^{-4}$ & $-3 \%$ \\
\hline RF voltage jitter & $\Delta V / V \lesssim 3 \%$ & $-3 \%$ \\
\hline
\end{tabular}

of the centroid jitter. Tolerances are listed in Table 16; they correspond to $3 \%$ luminosity reduction, which is similar to a $6 \%$ emittance increase. The tolerances on the power supplies are not severe, but the tolerances on the quadrupole vibration are tight and may be difficult to achieve. One may be able to use feedback to ease the tolerances. Unfortunately, beam-based feedback will be difficult at the relativoly high frequencies, and thus, we may need to develop active and passive damping systems for the individual quadrupole supports. 
Thus, to conclude, we note that the fundamental emittance dilutions are very small. Instead, the primary sources of emit tance dilution depend upon the alignment tolerances and the pulse-to-pulse stability: Furthermore, in a machine with weak filamentation, correction techniques can be used to substantially ease the alignment tolerances. 


\section{CHAPTER 4 CONCLUDING REMARKS}

Many future linear colliders are calling for normalized vertical emittances of $\gamma \epsilon_{y}=3 \times 10^{-8} \mathrm{~m}$-rad with aspect ratios $\epsilon_{x} / \epsilon_{y}$ of 100 . In this dissertation, we have investigated the tolerances and fundamental limitations on generating and preserving these very small vertical emittances; the results are then applied to the NLC linear collider design.

We have considered sources of emittance dilution in only two of the major subsystems of the linear collider, namely, the damping rings and the linear accelerators; wc have neglected dilutions that can occur in the bunch compressors and the collimation/final focus regions. Furthermore, we have neglected all multi-bunch issues; the NLC design calls for ten closely spaced bunches and thus multi-bunch effects are potentially very important and need to be examined.

We have treated these two subsystems, the damping rings and linacs, separately, dividing this dissertation into two major sections. Although the driving physics is the same in both regions, the sources of dilution differ in importance. Specifically, in the damping rings, the main sources of emittance dilution are non-conservative processes while, in the linacs, the primary limitations are due to conservative dilutions and pulse-to-pulse jitter; detailed discussions can be found in the respective summaries.

To conclude, we address the question: is it possible to generate and accelerate bunches with vertical emittances of $\gamma \epsilon_{y}=3 \times 10^{-8} \mathrm{~m}$-rad? The answer is certainly, yes; in the NLC design, the fundamental limitations are much smaller. But, the more important question, is it practical? The answer here depends upon the state of the hardware. But, it does seem practical, although some advances in hardware will be needed. In particular, to correct the emittance dilutions due to misalignments, we will need BPMs that have micron precision (reading-to-reading jitter) and we will need the ability to measure these beam emittances. In addition, we will need to develop a (cost-effective) method of reducing the pulse-to-pulse jitter; this will likely include feedback on the beam and the individual components.

Finally, we again note that dilutions in either the bunch compressors or the final focus regions were not considered. Furthermore, we have not examined the limitations due to multiple bunches and we have only considered the weak filamentation 
regime. All of these effects will increase the emittance dilution and need to be included in future work. In addition, many calculations throughout the dissertation were made using simple models which could be improved. This includes: the effect of ground motion in both the linacs and the damping rings, the wakefields in the damping ring, effects on the injected beam in the damping rings, the space charge coupling in the linacs, and finally, detailed simulations of the correction techniques, including "all" sources of error. 


\section{Appendix A \\ BEAM DYNAMICS: SURVEY}

In this appendix, we will briefly review the dynamics in an $e^{+} / e^{-}$accelerator. Detailed derivations of the equations and discussions of the particle motion can be found in any textbook on charged particle optics. Here, we will describe the salient physics and provide the necessary definitions so that one can follow the body of this dissertation. In particular, we will discuss the linear transverse and longitudinal motion and then we will consider the effects of the synchrotron radiation. Finally, we will discuss some of the issues pertinent to damping rings. The first portion of this appendix, Sections A.1-A.5, is a summary of Refs. 23, 62, and 104 while Section A.6 is mostly taken from Ref. 90.

\section{A.1 Coordinate System}

We will use a right-handed coordinate system: $(x, y, s)$, where $s$ is the distance along the accelerator and $x$ and $y$ are the horizontal and vertical coordinates. We always chose $y$ in the upward direction and $s$ in the direction of propagation; these then determine the $x$ direction. In addition, we will use the coordinate $z$ to describe the longitudinal position of particles within a bunch; $z$ has the same orientation as $s$, but it is referenced to the center of the bunch.

\section{A.2 Transverse Motion}

In this section, we will describe the transverse motion of the particles in a storage ring or transport line. We start by introducing the equations of motion and separating them into a closed orbit, the first order energy dependence, and the betatron motion about the central trajectory. Then we introduce the beta function, the phase advance, and the single particle invariant. Finally, we describe the beam ellipse which parameterizes a particle beam.

Equations of Motion

In a high energy accelerator, transverse magnetic fields are used to control the charged particle beam. The primary components are dipole, quadrupole, and sextupoie magnets; pole and ficld profiles for these magnets are illustrated in Fig. 47. Dipole magnets have a uniform field and are used to direct the beam trajectory while quadrupole magnets have fields that increase linearly with distance from the 

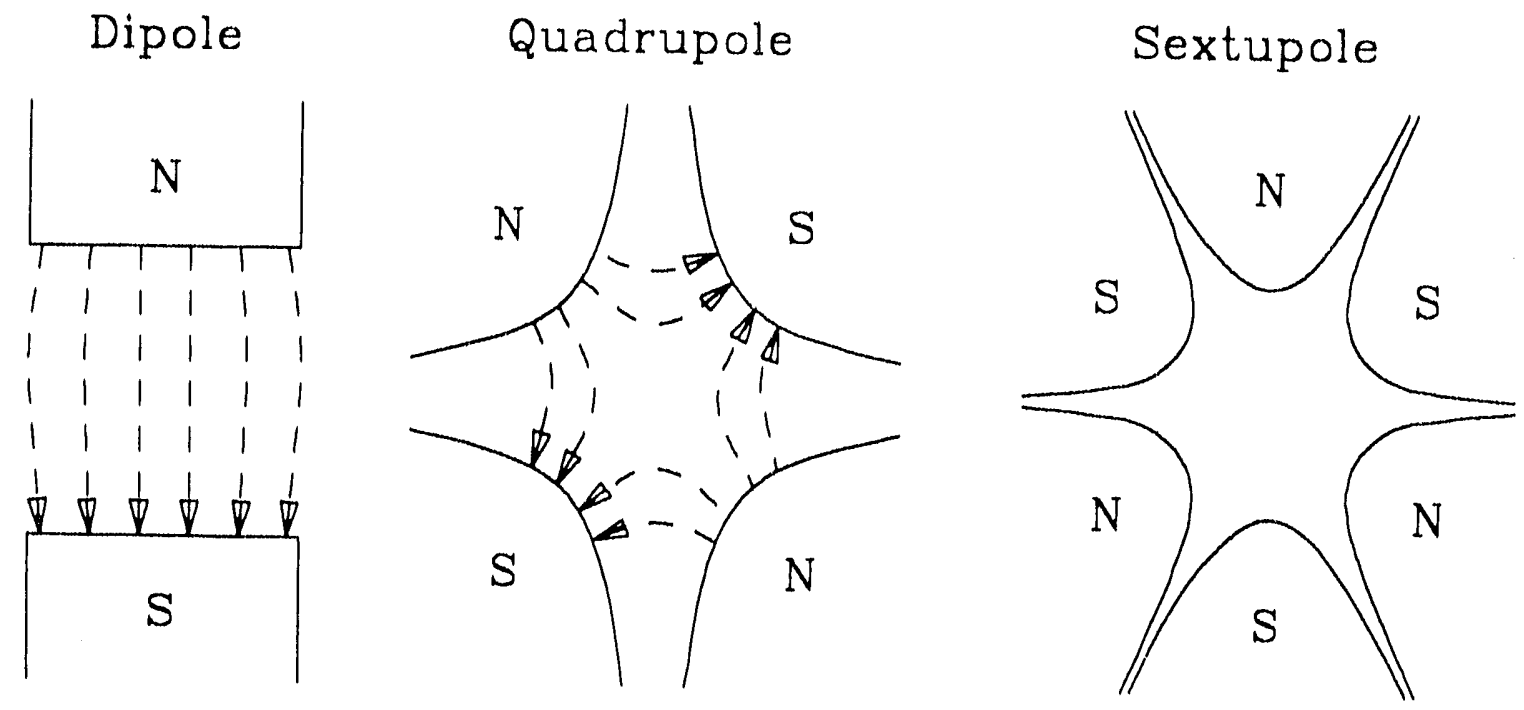

Fig. 47. Accelerator magnets.

magnetic center and thus they provide linear focusing. Finally, sextupole magnets and higher multipole magnets are used to correct nonlinear dependencies. In particular, sextupole magnets are used to correct the energy dependence of the focusing due to the quadrupoles.

In an accelerator, the longitudinal motion within the beam, referred to as the synchrotron motion, is usually much slower than the transverse. Thus, we can solve the transverse equations while lıolding the longitudinal motion fixed; the complications neglected in this approximation are discussed in Section 2.2.6. With this approximation and in the absence of synchrotron radiation and longitudinal acceleration, the equations of motion for a single particle can be written [100]

$$
\begin{gathered}
x^{\prime \prime}+(1-\Delta)\left[\left(K_{1}(s)+G^{2}(s)\right) x+\frac{K_{2}(s)}{2}\left(x^{2}-y^{2}\right)\right]= \\
\Delta G(s)+(1-\Delta) G_{x c}(s) \\
y^{\prime \prime}-(1-\Delta)\left[K_{1}(s) y+K_{2}(s) x y\right]=(1-\Delta) G_{y c}^{\prime}(s)
\end{gathered}
$$

where the primes denote derivatives with respeet to s, the azimuthal coordinate, and $\Delta$ is the relative energy deviation: $\Delta \equiv\left(p-p_{0}\right) / p$ where $p$ is the particle momentum 
and $p_{0}$ is the design momentum; to first-order $\Delta$ equals the more common parameter $\delta \equiv\left(p-p_{0}\right) / p_{0}$. In addition, $G$ is the main horizontal guide field which is the inverse of the local bending radius, $G_{x c}$ and $G_{y c}$ are the inverse bending radii of additional corrector or error dipole fields, and $K_{1}$ and $K_{2}$ are the normalized quadrupole and sextupole fields:

$$
G(s) \equiv \frac{1}{\rho(s)}=\frac{e}{p_{0}} B_{y} \quad K_{1}(s) \equiv \frac{e}{p_{0}} \frac{\partial B_{y}}{\partial x} \quad K_{2}(s) \equiv \frac{e}{p_{0}} \frac{\partial^{2} B_{y}}{\partial x^{2}} .
$$

With these definitions, positive $G_{x c, y c}$ causes a deflection in the positive $x$ or $y$ direction and positive $K_{1}$ corresponds to focusing in the horizontal plane.

At this point, we can separate the solutions into: (1) the on-energy $(\Delta=0)$ inhomogeneous solution, referred to as the central trajectory, (2) the first-order energy dependence of the central trajectory, referred to as the dispersion function, and (3) the homogeneous solution which is referred to as the betatron motion. Thus, $x=x_{c}+\delta \eta_{x}+x_{\beta}$ where $x_{c}$ is the central trajectory, $\eta_{x}$ is the dispersion function, and $x_{\beta}$ is the betatron motion, and the vertical motion is similar. With this substitution, the equations for the central trajectory are:

$$
\begin{aligned}
x_{c}^{\prime \prime}+\left(K_{1}+G^{2}\right) x_{c}+\frac{K_{2}}{2}\left(x_{c}^{2}-y_{c}^{2}\right) & =G_{x c} \\
y_{c}^{\prime \prime}-K_{1} y_{c}-K_{2} x_{c} y_{c} & =G_{y c} .
\end{aligned}
$$

Next, linear equations for the dispersion function and the betatron motion can be found by expanding about the central trajectory:

$$
\begin{aligned}
\eta_{x}^{\prime \prime}+\left(K_{1}+G^{2}\right) \eta_{x}+K_{2}\left(x_{c} \eta_{x}-y_{c} \eta_{y}\right) & = \\
G-G_{x c}+\left(K_{1}+G^{2}\right) x_{c} & +\frac{K_{2}}{2}\left(x_{c}^{2}-y_{c}^{2}\right) \\
\eta_{y}^{\prime \prime}-K_{1} \eta_{y}-K_{2}\left(x_{c} \eta_{y}+y_{c} \eta_{x}\right) & =-G_{y c}-K_{1} y_{c}-K_{2} x_{c} y_{c} .
\end{aligned}
$$

and

$$
\begin{aligned}
x_{\beta}^{\prime \prime}+(1-\Delta)\left[\left(K_{1}^{\prime}+G^{2}\right) x_{\beta}+K_{2}\left(x_{c} x_{\beta}-y_{c} y_{\beta}\right)\right]+\Delta K_{2}\left(\eta_{x} x_{\beta}-\eta_{y} y_{\beta}\right)=0 \\
y_{\beta}^{\prime \prime}-(1-\Delta)\left[K_{1} y_{\beta}+K_{2}\left(y_{c} x_{\beta}+x_{c} y_{\beta}\right)\right]-\Delta K_{2}\left(\eta_{y} x_{\beta}+\eta_{x} y_{\beta}\right)=0
\end{aligned}
$$

In this appendix, we will neglect the complications due to the sextupoles; these effects are discussed in Section 2.2 . 


\section{Betatron Motion}

If we neglect the sextupole terms, the equations for the betatron motion are similar to those of one-dimensional harnonic oscillators and can be expressed in an analogous form:

$$
\begin{aligned}
& x_{\beta}=\sqrt{2 J_{x} \beta_{x}(s)} \cos \left(\psi_{x}(s)+\phi_{x}\right) \\
& y_{\beta}=\sqrt{2 J_{y} \beta_{y}(s)} \cos \left(\psi_{y}(s)+\phi_{y}\right) .
\end{aligned}
$$

Here, $J_{x, y}$ and $\phi_{x, y}$ are constants of the motjon, $\beta_{x, y}(s)$ are the beta functions, and $\psi_{x, y}(s)$ are the phase advances given by:

$$
\psi_{x, y}(s) \equiv \int_{0}^{s} \frac{d s^{\prime}}{\beta_{x, y}\left(s^{\prime}\right)}
$$

In a storage ring, the beta function is chosen to be periodic but in a transport line there is room for ambiguity since one needs to define initial values or boundary conditions; we will discuss this further in Section 3.3.1.

Similarly, a storage ring has a well defined tune; the tune equals the phase advance around the ring divided by $2 \pi$ :

$$
\nu_{x, y} \equiv \frac{\psi_{x, y}(C)-\psi_{x, y}(0)}{2 \pi}
$$

where $C$ is the ring circumference. The tunes are very important in a storage ring since they locate the proximity of destructive resonances. In general, there is a resonance whenever $k \nu_{x}+l \nu_{y}+m \nu_{s}=n$, where $k, l, m$, and $n$ are integers and $\nu_{s}$ is the tune of the longitudinal motion.

In addition to the beta function and the phase advance, there are two other parameters that are used to describe a focusing lattice. For systems without acceleration, these are:

$$
\alpha_{x, y} \equiv-\frac{1}{2} \frac{d \beta_{x, y}}{d s} \quad \text { and } \quad \gamma_{x, y} \equiv \frac{1+\alpha_{x, y}^{2}}{\beta_{x, y}} \text {. }
$$

Note that one needs be careful since the symbols used to denote these functions also refer to the transverse damping rates and the relativistic factor $\gamma$; the context should clarify the meaning. 
Finally, we can also describe the linear betatron motion in terms of transport matrices $\mathbf{R}$ :

$$
\left(\begin{array}{c}
x(s) \\
x^{\prime}(s)
\end{array}\right)=\left(\begin{array}{ll}
R_{11}(0, s) & R_{12}(0, s) \\
R_{21}(0, s) & R_{22}(0, s)
\end{array}\right)\left(\begin{array}{c}
x(0) \\
x^{\prime}(0)
\end{array}\right) .
$$

This form is useful because the individual transport matrices can be constructed directly from the linear lattice elements: drifts, dipoles, and quadrupoles, and then multiplied together to form the transport matrix for the structure. We will frequently refer to the $R_{11}$ and $R_{12}$ matrix elements which, in terms of the lattice parameters, are

$$
\begin{aligned}
& R_{11}\left(s_{1}, s_{2}\right)=\sqrt{\frac{\beta_{2}}{\beta_{1}}} \sqrt{\frac{\gamma_{1}}{\gamma_{2}}}\left(\cos \Delta \psi+\alpha_{1} \sin \Delta \psi\right) \\
& R_{12}\left(s_{1}, s_{2}\right)=\sqrt{\beta_{1} \beta_{2}} \sqrt{\frac{\gamma_{1}}{\gamma_{2}}} \sin \Delta \psi,
\end{aligned}
$$

Here, the relativistic factor' $\gamma$ has been used to include the adiabatic damping that occurs as the beam is accelerated. Adiabatic damping occurs because as the beam is accelerated $p_{0}$ increases while the transverse momenta remain constant. Thus, in $x-x^{\prime}$ and $y-y^{\prime}$ phase spaces there is damping since $x^{\prime} \equiv(1+G x) p_{x} / p_{0}$ decreases. In this case, the betatron motion is still described by Eq. (A.2.6), but the definition of $\alpha$ must be changed to

$$
\alpha_{x, y} \equiv-\frac{1}{2} \frac{d \beta x, y}{d s}+\frac{\beta_{x, y}}{\gamma} \frac{d \gamma}{d s}
$$

and the single particle invariant decreases inversely with the beam encrgy.

Central Trajectory and Encrgy Dejendence

At this point, we can find the central trajectory about which the particles perform betatron oscillations. In a storage ring, the central trajectory is periodic and is referred to as the closed orbit. In an $e^{+} / e^{-}$ring, the particles damp towards this closed orbit. Neglecting the sextupoles, the periodic solution for $x_{c}$ is

$$
x_{c}(s)=\frac{\sqrt{\beta_{x}(s)}}{2 \sin \pi \nu_{x}} \int_{s}^{s+C^{\prime}} \sqrt{\beta_{x}\left(s^{\prime}\right)} \cos \left(\psi_{x}(s)-\psi_{x}\left(s^{\prime}\right)+\pi \nu_{x}\right) G_{x c}\left(s^{\prime}\right) d s^{\prime},
$$

with an analogous result for the vertical plane. In a transport line, the central trajectory depends upon the initial conditions $\left(a_{0}, x_{0}^{\prime}\right)$. Typically, these are chosen 
to be zero, in which case the contral trajectory is

$$
x_{c}(s)=\int_{0}^{s} \sqrt{\beta_{x}(s) \beta_{x}\left(s^{\prime}\right)} \sqrt{\frac{\gamma\left(s^{\prime}\right)}{\gamma(s)}} \sin \left(\psi(s)-\psi^{\prime}\left(s^{\prime}\right)\right) G_{x c}\left(s^{\prime}\right) d s^{\prime}
$$

the trajectory is the integral of the $R_{12}$ matrix element and the deflections $G_{x o} d s$.

Now, we can calculate the dispersion function $\eta_{x, y}$. This is the first-order change of the central trajectory with $\Delta$ (or $\delta$ ). In a storage ring, we have the periodic solution

$$
\begin{aligned}
\eta_{x}(s)=\frac{\sqrt{\beta_{x}(s)}}{2 \sin \pi \nu_{x}} \int_{s}^{s+C} \sqrt{\beta_{x}\left(s^{\prime}\right)} \cos \left(\psi_{x}(s)-\psi_{x}\left(s^{\prime}\right)+\pi \nu_{x}\right) \\
\quad \times\left[G^{\prime}-G_{x c}+\left(\Lambda_{1}+G^{2}\right) x_{c}\right] d s^{\prime} .
\end{aligned}
$$

while in a transport line we have

$$
\eta_{x}(s)=\int_{0}^{s} \sqrt{\beta_{x}(s) \beta_{x}\left(s^{\prime}\right)} \sqrt{\frac{\gamma\left(s^{\prime}\right)}{\gamma(s)}} \sin \left(\psi(s)-\psi\left(s^{\prime}\right)\right)\left[G-G_{x c}^{\prime}+\left(K_{1}+\left(g^{\prime 2}\right) x_{c}\right] d s^{\prime}\right.
$$

where we have assumed that the initial values $\left(\eta_{x 0}, \eta_{x 0}^{\prime}\right)$ are zoro. Again, there are analogous results for the vertical plane.

Finally, we calculate the variation of the phase advance with energy; this is referred to as the chromaticity. As the particle energy increases above the design energy, the focusing, and thus the phase advance, becomes weaker. In a storage ring, the uncoupled chromaticity is

$$
\xi_{x, y} \equiv \frac{d \nu_{x, y}}{d p / p_{0}}=\frac{\mp 1}{4 \pi} \oint\left(\Lambda_{1}-\Lambda_{2} \eta_{x}\right) \beta_{x, y} d s^{\prime}
$$

where the integral is calculated around the ring and the $(-)$ sign applies to $\xi_{x}$ and the $(+)$ sign applies to $\xi_{y}$. Without the sextupoles, a storage ring naturally has a negative chromaticity. This can lead to coherent instabilities and thus the chromaticity is corrected to be zero or slightly positive with the sextupole magnets located in regions of horizontal dispersion. 
Emittance, Beam Ellipse, and Machine Ellipse

So far we have only discussed the trajectory of a single particle, Actually, we have a beam of particles which all have different amplitudes and phases. We quantify the phase space volume occupled by the beam in terms of the normalized emittance $\gamma \epsilon$. The normalized emittance is a useful quantity because in a conservative system, which a linear accelerator approximates, the six-dimensional phase space volume $\left(x, p_{x}, y, p_{y}, z, \Delta E\right)$ enclosing the bean is invariant. Furthermore, if the three degrees of freedom are uncoupled, then the phase space area of each degree of freedom is also conserved.

Throughout this dissertation, we will refer to the rms emittance. This is a parameterization of the phase space volume using the second moments of the beam distribution. If the forces are linear, the six-dimensional ims emittance is conserved and, if the planes are uncoupled, exch two-dimensional rms emittance is conserved. In the transverse planes, the two-dimensional normalized rms emittance is equal to

$$
\gamma \epsilon_{x}=\gamma \sqrt{\left\langle x_{\beta}^{2}\right\rangle\left\langle x_{\beta}^{\prime 2}\right\rangle-\left\langle x_{\beta} x_{\beta}^{\prime}\right\rangle^{2}}
$$

where the angle brackets denote an average over the beam particles. More generally, the rms emittance can be expressed as the determinant of the beam matrix $\sigma$ :

$$
\gamma:=\gamma \operatorname{det} \sigma \quad \sigma_{i j}=\left\langle x_{i} x_{j}\right\rangle
$$

where $x_{i}$ are components of the vector: $\left(x_{\beta}, x_{\beta}^{\prime}, y_{\beta}, y_{\beta}^{\prime}, z, \delta\right)$.

The moments of the bean distribution, $\left\langle x_{\beta}^{2}\right\rangle,\left\langle x_{\beta}^{\prime 2}\right\rangle$, and $\left\langle x_{\beta} x_{\beta}^{\prime}\right\rangle$, define the beam ellipse in the $x-x^{\prime}$ phase space that can be used to further parameterize the beam. In particular, the area of the beam ellipse is related to the rms emittance as Area $=\pi \epsilon$. Furthermore, $e^{+} / e^{-}$beams usually have gaussian transverse distributions in which case the beam ellipse describes a contour of constant density that encloses $39 \%$ of the beam.

In general, we can express these moments in terms of the beam parameters $\alpha^{\star}$, $\beta^{\star}$, and $\gamma^{\star}$ :

$$
\left\langle x_{\beta}^{2}\right\rangle=\beta_{x}^{\star} \epsilon_{2} \quad\left\langle x_{\beta}^{\prime}{ }^{2}\right\rangle=\gamma_{x}^{*} \epsilon_{x} \quad\left\langle x_{\beta} x_{\beta}^{\prime}\right\rangle=-\alpha_{x}^{\star} \epsilon_{x}
$$

where the beam parameters are similar, but not necessarily equal, to the lattice parameters; the lattice paraneters describe the focusing lattice while the beam parameters describe the beam. 


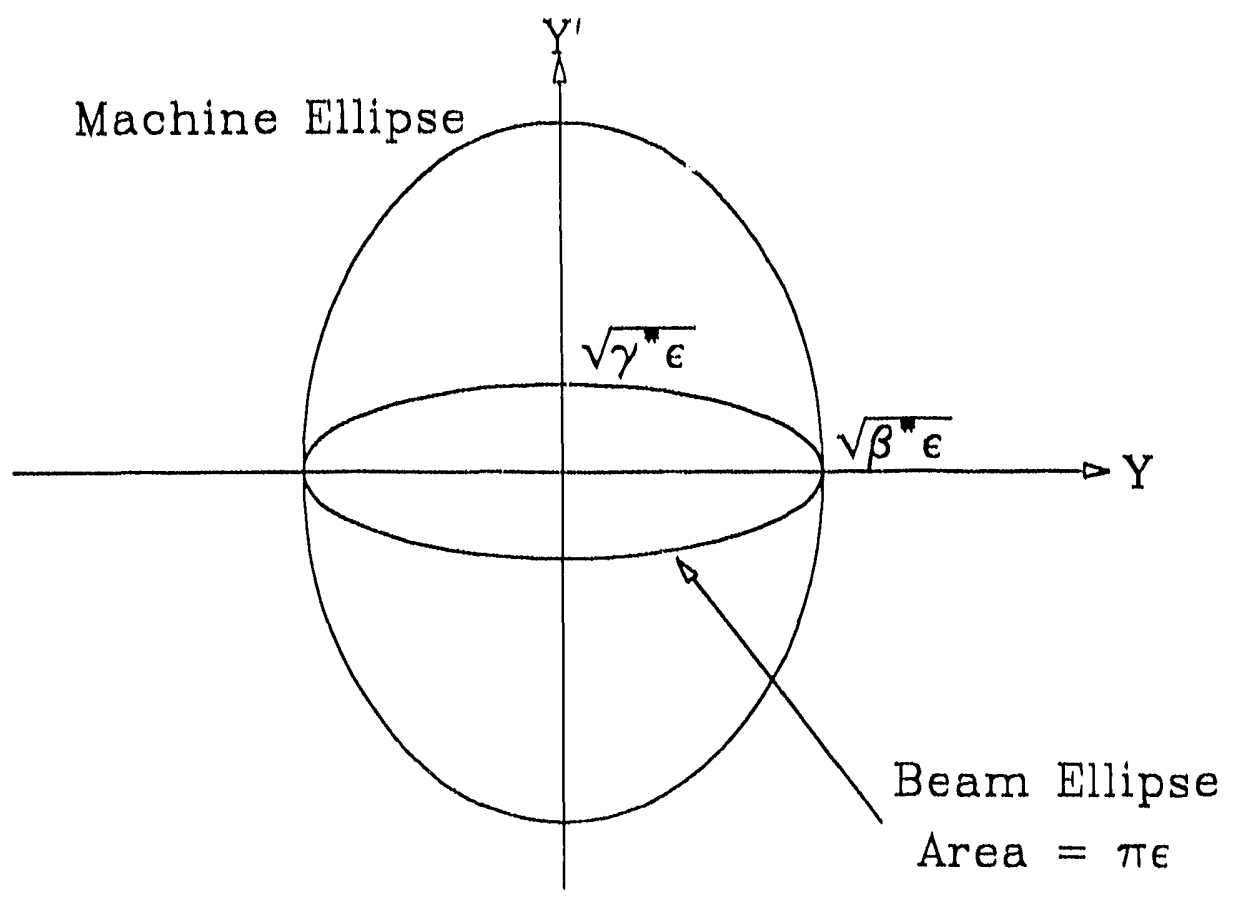

Fig. 48. Beam and machine ellipses for an unmatched beam.

At this point, we introduce the machine ellipse. The single particle invariant, introduced in $\mathrm{Eq}$. (A.2.6), can be expressed in terms of $x_{\beta}$ and $x_{\beta}^{\prime}$ the lattice parameters:

$$
J_{x}=\frac{1}{2}\left[\gamma_{x} x_{\beta}^{2}+2 \alpha_{x} x_{\beta} x_{\beta}^{\prime}+\beta_{x} x_{\beta}^{\prime 2}\right],
$$

and $J_{y}$ is similar. Thus, particles with constant $J_{x, y}$ are distributed on an ellipse in $x * x^{\prime}$ phase space which is defined by the single particle invariant and the lattice parameters; this is referred to as the machine ellipse.

In general, the beam ellipse is inscribed in the machine ellipse. If the beam is matched to the focusing lattice, then the beam and the machine ellipses are equal. In this case, the beam parameters are equal to the lattice parameters and we can express the emittance in terms of the single particle invariant: $\epsilon_{x, y}=\left\langle J_{x, y}\right\rangle$.

This parameterization is not valid if the beam is not matched to the structure. In such a case, the beam ellipse is inscribed in the machine ellipse; this is illustrated in Fig. 48. If all particles in the beam lave the same phase advance, then the beam ellipse rotates coherently within the machine ellipse, but if there is a spread in the phase advance, the beam will filument and fill all of the machine ellipse and there is a corresponding rms enitance growth; filamentation is discussed further in Section 3.3.1. 
Finally, it is important to realize that the rms normalized emittance is not necessarily conserved; filamentation i. one illustration of this. In particular, nonlinear fields or coupling can distort the beam ellipse so that the second moments do not accurately reflect the phase space volume. Regardless, accelerators are fairly linear machines and the rms emittance provides a useful measure of the actual beam emittance.

\section{A.3 Longitudinal Motion}

Now, we need to discuss the longitudinal motion. There are two ways for the particles to change their relative longitudinal positions: velocity differences and path differences. The velocity difference between two ultra-relativistic particles leads to a longitudinal position change of

$$
\Delta z \simeq \frac{s \delta}{\gamma^{2}}
$$

where $\delta$ is the relative energy difference, $\gamma$ is the relativistic factor, and $s$ is the distance traveled. When $\gamma$ is large, this tends to be a negligible effect.

The path length for a particle can be written

$$
L=\int\left(1+\frac{x}{\rho(s)}\right)\left[1+\left(\frac{d x}{d s}\right)^{2}+\left(\frac{d y}{d s}\right)^{2}\right]^{\frac{1}{2}} d s
$$

where we have assumed that the only significant bending occurs in the horizontal plane. To first-order, only the curvature term is important and thus the path length difference is

$$
\Delta z=\int \frac{\Delta x}{\rho(s)} d s
$$

where $\Delta x$ can be due to a betatron oscillation or the dispersion. Notice that $\Delta z$ depends upon the bending radius and thus the longitudinal position is essentially fixed in a linear accelerator where $\rho \rightarrow \infty$.

The dependence of $\Delta z$ on the betatron amplitude couples the longitudinal plane to the transverse. This will be discussed further in Section 2.2.6, but, on average, the effect of a betatron oscillation is small; the betatron oscillation has a different phase every turn and thus the effect averages to zero. This leaves the effect of 
the dispersion which can be written: $\Delta z=\delta \alpha_{m} C$, where $\alpha_{m}$ is the momentum compaction factor:

$$
\alpha_{m} \equiv \frac{d C / C}{d p / p_{0}}=\frac{1}{C} \int_{0}^{C} \frac{\eta_{x}}{\rho} d s
$$

At this point, we need to discuss the radio-frequency (RF) acceleration system. The RF system provides longitudinal electric fields that can accelerate or decelerate the particles. In an $e^{+} / e^{-}$ring, the RF system is needed to replace the energy emitted as synchrotron radiation while in a linac the RF is used to accelerate the beam. Usually, the system consists of resonant RF cavities that are powered by microwave sources. Assuming a sinusoidal RF, the accelerating voltage across a cavity can be written

$$
V_{\mathrm{RF}}=V_{0} \sin \omega_{\mathrm{RF}} t
$$

where $\omega_{R F}$ is the RF frequency.

In a high energy linac, the longitudinal positions of the particles do not change since there is no bending. Thus, the particle energy depends upon the longitudinal position and the phase of the RF voltage seen by the beam; usually the beam is being accelerated and it is placed near the crest of the RF vcltage. We can express the voltage as

$$
V_{\mathrm{RF}}^{\prime}=V_{0} \sin \left(z k_{\mathrm{RF}}+\phi_{s}\right)
$$

where $k_{\mathrm{RF}}$ is the RF wavenumber and $\phi_{s}$ is the synchronous phase: $\phi_{s}=\pi / 2$ for maximum acceleration.

In a storage ring, the situation is clifferent. Here, $\omega_{R F}$ is chosen to be an integral multiple of the revolution frequency. Thus, we can express the voltage seen by a circulating particle as $V_{0} \sin \left(z\left(t_{n}\right) k_{\mathrm{RF}}+\phi_{s}\right)$ where $t_{n}$ is time at which the particle passes the cavity on turn $n$. Thus, the change in $z$ depends upon the energy and the change in energy depends upon $z$. Since the changes per turn are very small, we can write a differential equation for the longitudinal motion:

$$
z^{\prime \prime}+\frac{\alpha_{m}}{C} \frac{c V_{0}}{E_{0}} \sin \left(k_{\mathrm{RF}} z+\phi_{s}\right)=\frac{\alpha_{m}}{C^{\prime}} \frac{\Delta E_{\mathrm{rad}}}{E_{0}}
$$

which we can express as

$$
z^{\prime \prime}+\left(\frac{\alpha_{m}}{C} \frac{e V_{0}}{E_{0}} k_{\mathrm{RF}} \cos \phi_{s}\right) z=-\frac{\alpha_{m}}{C} \frac{\epsilon V_{0}^{\prime}}{E_{0}} k_{R F} \sin \phi_{s}+\frac{\alpha_{m}}{C^{\prime}} \frac{\Delta E_{\mathrm{rad}}}{E_{0}}
$$


Here, $\Delta E_{\mathrm{rad}}$ is the average energy lost on one turn.

The r.h.s. of Eq. (A.3.8) determines the synchronous phase while the 1.h.s. determines the synchrotron motion. If we linearize this and make a smooth approximation, which is valid because the synchrotron motion is slow, we can express the synchrotron motion as

$$
\begin{aligned}
& \delta(s)=\delta_{0} \cos \left(2 \pi \nu_{s} \frac{s}{C}+\phi_{0}\right) \\
& z(s)=\frac{\alpha_{m} C}{2 \pi \nu_{s}} \delta_{0} \sin \left(2 \pi \nu_{s} \frac{s}{C}+\phi_{0}\right),
\end{aligned}
$$

where $\delta_{0}$ and $\phi_{0}$ are the constants of the motion and $\nu_{s}$ is the synchrotron tune:

$$
\nu_{s}=\frac{1}{2 \pi}\left(\alpha_{m} C \frac{e V_{0}}{E_{0}} k_{R F} \cos \phi_{s}\right)^{\frac{1}{2}}
$$

\section{A.4 Radiation Damping ${ }^{(204)}$}

When an ultra-relativistic particle is accelerated, it emits synchrotron radiation. The emission of the radiation is intrinsically a quantum mechanical phenomena and the time of emission and the energy of the emitted photon are random. However, provided that the expected energy of the photons is small compared to the particle energy, the photon distribution can be accurately calculated using classical electromagnetic theory. In this section, we will only consider effects of the average power radiated. Thus, we neglect the discrete nature of the photons and treat the radiation as a classical field that is emitted continuously; the quantum nature of the radiation is discussed in the next section.

As the particles orbit the ring, they are deflected by the dipole magnets and they radiate. The average power radiated around the ring $\overline{P_{\gamma}}$ can be expressed

$$
\overline{P_{9}}=\frac{2}{3} \frac{r_{0} e^{2}}{m^{3} c^{3}} E^{2} \overline{B^{2}(s)}
$$

where $m$ is the electron mass, $E$ is the particle energy, and $\overline{B^{2}}$ is the average value of the magnitude of the transverse magnetic field squared. 
In general, particles with higher energies radiate more than particles with lower energies. This leads to an exponential damping of the longitudinal phase space. The longitudinal damping rate is

$$
\alpha_{\epsilon} \equiv \frac{1}{\Delta E} \frac{d \Delta E}{d t}=\frac{1}{2} \frac{d \overline{P_{\gamma}}}{d E}
$$

When evaluating the derivative of $\overline{P_{\gamma}}$ we have to include three effects: the dependence on the energy of the particle, the dependence on the revolution time which varies with energy, and the dependence on the magnetic field which varies with the orbit which is also a function of the energy. When all the terms are included correctly, we find

$$
\alpha_{c}=\frac{1}{\tau_{\epsilon}}=\mathcal{J}_{\epsilon} \frac{\overline{P_{\gamma}}}{2 E}=\mathcal{J}_{\epsilon} \frac{C_{\gamma}}{4 \pi} \frac{E_{0}^{3}}{T_{0}} \oint G^{2} d s,
$$

where $C_{\gamma}=8.85 \times 10^{-5} \mathrm{~m}-\mathrm{GeV}^{-3}, T_{0}$ is the revolution time, and $\mathcal{J}_{\epsilon}$ is the longitudinal damping partition number:

$$
\mathcal{J}_{\epsilon}=2+\frac{\oint \eta_{x} G\left(G^{2}+2 K_{1}\right) d s}{\oint G^{2} d s} .
$$

Note that $\alpha_{\epsilon}$ is the damping rate of $z$ or $\delta$; the longitudinal emittance damps at a rate of $2 \alpha_{\ell}$.

In the transverse planes, the radiation decreases the transverse momenta $p_{x}$ and $p_{y}$ and again leads to an exponential damping. In this case, the damping rates are

$$
\alpha_{x, y}=\frac{1}{\tau_{x, y}}=\mathcal{J}_{x, y} \frac{\overline{P_{\gamma}}}{2 E}=\mathcal{J}_{x, y} \frac{C_{\gamma}}{4 \pi} \frac{E_{0}^{3}}{T_{0}} \oint G^{2} d s,
$$

where $\mathcal{J}_{x, y}$ are the transverse damping partition numbers which, if the ring is built in the horizontal plane, are

$$
\mathcal{J}_{x}=1-\frac{\oint \eta_{x} C_{r}\left(G^{2}+2 K_{1}\right) d s}{\oint G^{2} d s} \quad \text { and } \quad \mathcal{J}_{y}=1
$$

Notice that the sum of the damping partitions is a constant: $\mathcal{J}_{e}+\mathcal{J}_{x}+\mathcal{J}_{y}=4$. This is a statement of the Robinson theorem ${ }^{\text {[93) }}$ and is valid whenever a negligible quantity of synchrotron radiation is emitted in regions where the external fields are time dependent, i.e., the RF cavities. ${ }^{[6,2]}$ 


\section{A.5 Quantum Excitation ${ }^{(104)}$}

The synchrotron radiation is actually emitted as a series of photons which change the particle's energy in discrete steps; the emission can be considered instantaneous in comparison to the particle motion. Since the emission of a photon is a stochastic process, the radiation will introduce noise which leads to diffusion. This causes the beam emittances to grow until the diffusion is balanced by the radiation damping.

As discussed, the radiation damping is due to the mean synchrotron radiation power emitted. In contrast, the quantum excitation is due to higher moments of the photon distribution. We can express the synchrotron radiation power as the rate of emission multiplied by the expected photon energy:

$$
\mathcal{N}\langle u\rangle=P_{\gamma}(s)
$$

where $\mathcal{N}$ is the rate of emission, $u$ is the photon energy, and $P_{\gamma}$ is given by Eq. (A.4.1). To calculate the diffusion effects, we will need the second moment $\left\langle u^{2}\right\rangle$ which is

$$
\mathcal{N}\left\langle u^{2}\right\rangle=\frac{55}{16 \sqrt{3}} \frac{\hbar e}{m} \gamma^{2} B(s) P_{\gamma}(s)
$$

In the longitudinal plane, these fluctuations directly increase the energy spread. The particles are performing synchrotron oscillations, obeying Eq. (A.3.9), and if a particle emits a photon, the oscillation changes to

$$
\delta(s)=\delta_{0} \cos \left(2 \pi \nu_{s} \frac{s}{C}+\phi_{0}\right)+\delta_{\mathrm{rad}} \cos \left(2 \pi \nu_{s} \frac{s}{C}+\phi_{\mathrm{rad}}\right),
$$

and the amplitude of the oscillation becomes: $\delta^{2}=\delta_{0}^{2}+\delta_{\text {rad }}^{2}+2 \delta_{0} \delta_{\text {rad }} \cos \left(\phi_{0}-\phi_{\text {rad }}\right)$. Since the time of emission is not correlated with the synchrotron phase $\phi_{0}$, the expected amplitude grows at a rate

$$
\frac{d\left\langle\delta^{2}\right\rangle}{d t}=\frac{\mathcal{N}\left\langle u^{2}\right\rangle}{E_{0}^{2}}
$$

Now, we can find the equilibrium amplitude by equating the expected growth with the radiation damping:

$$
\frac{d\left\langle\delta^{2}\right\rangle}{d t}=2\left\langle\delta^{2}\right\rangle a_{\epsilon}=\frac{\mathcal{N}\left\langle u^{2}\right\rangle}{E_{0}^{2}}
$$

Finally, we average around the ring and we find the rms energy spread by averaging over all of the particles. This introduces a factor of one half, yielding an rms 
energy spread and an rms bunch length of:

$$
\sigma_{\epsilon}^{2}=C_{q} \frac{\gamma^{2}}{\mathcal{J}_{\epsilon}} \frac{\oint\left|G^{3}\right| d s}{\oint G^{2} d s} \quad \sigma_{z}=\frac{\alpha_{m} C}{2 \pi \nu_{s}} \sigma_{\epsilon}
$$

where $C_{q}$ is nearly equal to the Compton wavelength of the electron: $C_{q}=3.84 \times$ $10^{-13}$ meters.

In the transverse planes, there are two effects: (1) the transverse planes are coupled to the longitudinal through the dispersion function and (2) the radiation has a finite opening angle that gives the particle small transverse kicks. Here, we will only discuss the horizontal plane; the vertical plane is discussed in Section 2.2.

In the horizontal plane, the effect of the radiation opening angle is insignificant compared to the coupling due to the horizontal dispersion. When a particle radiates a photon, it's energy changes. Since the periodic trajectory around the ring is energy dependent, this implies that the particle oscillates about a new central trajectory. Furthermore, since the particle's physical transverse position does not change, the amplitude of the betatron oscillation must change. Specifically, if a photon of energy $u$ is radiated, the change in the betatron motion is:

$$
\Delta x_{\beta}=-\frac{u}{E_{0}} \eta_{x} \quad \Delta x_{\beta}^{\prime}=-\frac{u}{E_{0}} \eta_{x}^{\prime}
$$

At this point, we find the change in the single particle invariant $J_{x}$. Since the photon emission is not correlated with the betatron phase, we find an expected change

$$
\left\langle\Delta J_{x}\right\rangle=\frac{1}{2} \frac{\left\langle u^{2}\right\rangle}{E_{0}^{2}} \mathcal{H}_{x}
$$

where

$$
\mathcal{H}_{x} \equiv \gamma_{x} \eta_{x}^{2}+2 \alpha_{x} \eta_{x} \eta_{x}^{\prime}+\beta_{x} \eta_{x}^{2}
$$

Now, the procedure is identical to that used in the longitudinal plane; we find the equilibrium value by equating the damping with the diffusion and then average over all the particles to find the rms value. This yields

$$
\gamma \epsilon_{x}=\frac{\gamma}{4} \frac{\oint \mathcal{N}\left\langle u^{2}\right\rangle \mathcal{H}_{x} d s}{E_{0}^{2} \alpha_{x} T_{0} c}=C_{q} \frac{\gamma^{3}}{\mathcal{J}_{x}} \frac{\oint\left|G^{3}\right| \mathcal{H}_{x} d s}{\oint G^{2} d s} .
$$

Finally, we need to discuss the equilibrium particle distribution resulting from the synchrotron radiation. Many photons are radiated within a damping time; 
roughly $\gamma / 100$ photons are radiated per radian of curvature while the damping times are usually many hundreds of turns. Thus, using the Central Limit Theorem and assuming linear forces, we find that the equilibrium beam distribution is gaussian in each of the six coordinates: $\left(x_{\beta}, x_{\beta}^{\prime}, y_{\beta}, y_{\beta}^{\prime}, z, \delta\right)$. Alternately, this can expressed in terms of the single particle invariants which have exponential distributions:

$$
P_{\mathrm{SR}}\left(J_{x, y}\right)=\frac{e^{-J_{x, y} / \epsilon_{x, y}}}{\epsilon_{x, y}}
$$

\section{A.6 Damping Ring Parameters}

At this point, we will specialize to the case of damping rings. The two main parameters of a damping ring are the equilibrium emittance and the damping times. These determine the emittance of the extracted beam:

$$
\epsilon(t)=\epsilon_{i} e^{-2 t / r}+\left(1-e^{-2 t / \tau}\right) \epsilon_{0}
$$

where $\epsilon_{i}$ is the emittance of the injected beam and $\epsilon_{0}$ is the equilibrium ring emittance. Here, $\tau$ is the horizontal or vertical damping time, and $t$ is the time the particle bunch is in the ring.

To illustrate the determination of these parameters, we will use numbers from the NLC design described in Appendix B. The design requires that the extracted beam have normalized emittances of $\gamma \epsilon_{x} \leq 3 \times 10^{-6}$ and $\gamma \epsilon_{y} \leq 3 \times 10^{-8}$. Here, we assume an injected beam emittance of $\gamma \epsilon_{i}=3 \times 10^{-3}$, which is realistic for a positron beam and over an order of magnitude too large for an electron beam. Thus the vertical emittance needs to be decreased by five orders of magnitude. Damping the bunch for seven vertical damping times will reduce the first term of Eq. (A.6.1) by six orders of magnitude; we damp excessively to ease the tolerances on the equilibrium vertical emittance. The limit on the vertical equilibrium emittance of the ring is then

$$
\gamma \epsilon_{y 0} \leq 2.7 \times 10^{-8} \mathrm{~m}-\mathrm{rad}
$$

In a storage ring built in the horizontal plane the vertical emittance is mainly determined by the coupling between the horizontal and vertical planes. The tolerances necessary to achieve this limit are discussed in Chapter II. 
The damping times required in the ring are determined from the desired repetition rate $(360 \mathrm{~Hz})$, the number of damping times per bunch (7), and the number of batches of bunches stored in the ling at once $\left(N_{6}\right)$ :

$$
\tau_{x}, \tau_{y} \leq \frac{1}{f_{\text {rep }}} \frac{N_{b}}{\text { of damping times }}=N_{b} 0.397 \mathrm{~ms}
$$

The maximum number of batches stored in the ring is limited by the kickers needed for injection/extraction. We assume that the time for the kickers to turn on, extract/inject a batch, and turn off is less than $100 \mathrm{~ns} .^{(25)}$ Thus the batches must be separated by at least $50 \mathrm{~ns}$. Since the number of batches is roughly proportional to the size of the ring we can define an effective damping time as

$$
\tau_{\mathrm{eff}} \equiv \tau \frac{T_{\mathrm{sep}}^{\prime}}{T_{0}^{\prime}} \leq 0.397 \mathrm{~ms}
$$

where $T_{()}$is the revolution time of the ring and $T_{\text {sep }}$ is the separation of the batches.

In general, the horizontal damping time is less than or equal to the vertical, $\mathcal{J}_{x} \geq 1$. Thus, only the vertical damping time is limited by $\mathrm{Eq}$. (A.6.3) and the horizontal emittance of the extracted beam is very nearly ecual to the horizontal emittance of the ring:

$$
\gamma \epsilon_{x 0} \leq 3 \times 10^{-6} \mathrm{mrad}
$$

Equations (A.6.4) and (A.6.5) determine the basic parameters. Initially, to study these parameters, we ignore the option of damping wigglers and reversed bending magnets and we ignore the effect of intrabeam scattering. In this case, we can write simple expressions for $\gamma \epsilon_{x 0}$ and $\tau_{y \text { eff }}$, the two quantities we want to minimize:

$$
\begin{gathered}
\tau_{y \text { eff }}=1.69 \times 10^{14} \frac{T_{\text {sep }}}{\gamma^{3} G_{B}^{1}}=\frac{2.88 \times 10^{12} T_{\text {sep }}}{B_{0}\left[K^{\prime} G\right] \gamma^{2}} \\
\gamma \epsilon_{x 0}=3.84 \times 10^{-13} \frac{\gamma^{3}}{\mathcal{J}_{x}} \overline{\mathcal{H}_{x}} G_{B}=\frac{65 T_{\text {sep }}}{\tau_{y \text { eff }}} \frac{\overline{\mathcal{H}_{x}}}{\mathcal{J}_{x}} .
\end{gathered}
$$

Here, $G_{B}$ is the inverse bending radius of the bend magnets, $\overline{\mathcal{H}}_{x}$ is the average of $\mathcal{H}_{x}$ over the bending magnets, $B_{0}$ is the magnetic ficld in kilo-gauss, and we have assumed that $\mathcal{J}_{y}=1$. Finally, $T_{\mathrm{se}}$, is the batch separation which is determined by the fast injection/extraction kicker's. 
Equations (A.6.6) and (A.6.7) show that the emittance of a ring can be decreased by reducing the dispersion in the bend magnets, reducing the strength of the bends, or decreasing the energy of the ring. Unfortunately, the damping times are increased by reducing the bend magnet strength or decreasing the energy of the ring. This implies that the dispersion in the bends is the only free parameter. Unfortunately, as we will see, it is also constrained.

At this point, we need to discuss three additional parameters which constrain the design: (1) collective limitations, (2) the energy of the ring, and (3) the dynamic aperture. In general, the collective limitations are eased when the momentum compaction factor $\alpha_{m}$ is large. For example, the threshold for the longitudinal microwave instability, also called turbulent bunch lengthening, occurs at a bunch intensity

$$
N_{\text {thresh }} \approx \frac{(2 \pi)^{3 / 2} E_{0} \sigma_{c}^{2} \sigma_{z} \alpha_{m}}{e^{2} c(Z / n)_{\mathrm{eff}}}
$$

where $(Z / n)_{\text {eff }}$ is the effective longitudinal impedance. To maximize the charge per bunch, one wants to reduce the impedance and increase the longitudinal emittance, the beam energy, and the momentum compaction factor. Of course, the longitudinal emittance is constrained by the linear collider requirements, and, as we will discuss, the energy is also. Thus, this only leaves the momentum compaction factor which needs to be maximized.

Next, we would prefer to have the ring energy low. There are several reasons for this: (1) it keeps the ring cost lower, (2) it keeps the normalized longitudinal emittance small, and (3) it makes bunch compression easier. The NLC requires that the damping ring bunch be compressed longitudinally by, roughly, a factor of 100 . Since one does not want an uncorrelated energy spread much greater than $1 \%$ in the linac, we need to perform at least a portion of the bunch compression at an energy 10 times that of the damping ring. Unfortunately, at higher energies it becomes more difficult to perform the compression without degrading the beam emittances.

Finally, we need to mention the dynamic aperture. The dynamic aperture of the ring is a function of the sextupoles needed to correct the chromaticity. To prevent particle losses the dynamic aperture should be many times the injected beam size. Unfortunately, rings with small emittances tend to have high tunes and large uncorrected chromaticities. This makes the desired dy'namic aperture difficult, to achieve, and we would like to choose a focusing lattice which naturally has a large dynamic aperture. 
Obviously, these constraints are not necessarily consistent with achieving the required emittances and damping rates. For example, in the NLC design, the ring energy must be greater than $2.2 \mathrm{GeV}$ to achieve the damping, assuming iron magnets (non-superconducting) with salurated magnetic fields of $20 \mathrm{KG}$; this is higher than the desired energy. Fortunately, the damping requirements can be eased by using damping wigglers, reverse bending magnets, or a pre-damping ring to reduce the incoming emittance. In addition, it is common practice to use combined function bending magnets to increase $\mathcal{J}_{x}$ at the expense of $\mathcal{J}_{\mathfrak{f}}$ this decreases the horizontal ernittance. Detailed discussions of these choices in damping ring designs can be found in Refs. 13, 22, 36, 37, 90, 119, and 130. 


\section{Appendix B NLC DAMPING RING DESIGN}

Details of the current NLC damping ring design are described in Refs. 90 and 91. In addition, Ref. 90 also discusses the considerations that led to the design. Here, we will summarize the current stato of the design. To do so, we first list the original design goals and then we briefly describe the current lattice. Finally, we will comment on a few of the potential limitations of the design.

\section{B.1 Original Design Goals}

- $\gamma \epsilon_{x} \lesssim 3 \times 10^{-6} \mathrm{mrad}$ and $\gamma \epsilon_{y} \lesssim 3 \times 10^{-8} \mathrm{mrad}$.

- Operation at $360 \mathrm{~Hz}$.

- $\gamma \epsilon_{i}=3 \times 10^{-3} \mathrm{mrad} @$ injection - this necessitates damping for $\approx 7$ vertical damping times.

- Separate batches by $z 50$ ns for the injection/extraction kickers.

- Minimize wigglers d'e to cost and non-linearity.

- Leave more space between magnets than in the SLC damping rings.

- Achieve a conservative design.

Some of these goals are summarized in Table 17, which compares the goals of the NLC damping ring with the SLC positron damping ring design. The primary difference between the two rings is that the NLC ring needs to achieve a vertical emittance almost three orders of magnitude smaller than the SLC ring at twice the repetition rate. To achieve this damping rate, the ring needs to damp many batches of bunches at the same time. A single batch of 10 bunches is extracted on one kicker pulse while the remaining batches continue damping and an additional batch is injected to replace the extracted one.

The other main difference between the SLC and NLC damping rings is the very small vertical emittance specified for the NLC ring. This small vertical emittance sets limits on the alignment tolerances of the damping ring. While these tolerances are small ( $50-100 \mu \mathrm{m}$ vertical alignment), they are not thought to present a serious limitation. 
Table 17. Basic parameters of the SLC and NLC damping rings.

\begin{tabular}{|c|c|c|}
\hline & NLC & SLC \\
\hline Energy & $1 \sim 2 \mathrm{GeV}$ & $1.15 \mathrm{Gev}$ \\
\hline Injected emittance, $\gamma \epsilon_{i}$ & $3 \mathrm{mmrad}$ & $10 \mathrm{mmrad}$ \\
\hline Extracted emittance, $\gamma \epsilon_{x}$ & $3.0 \mu \mathrm{mrad}$ & $26 \mu \mathrm{mrad}$ \\
\hline Extracted emittance, $\gamma \epsilon_{y}$ & $30 \mathrm{mmrad}$ & $26 \mu \mathrm{mrad}$ \\
\hline Repetition rate & $360 \mathrm{~Hz}$ & $180 \mathrm{~Hz}$ \\
\hline Bunch length & $4-5 \mathrm{~mm}$ & $5 \mathrm{~mm}$ \\
\hline
\end{tabular}

\section{B.2 Current Design}

The basic layout of our current damping ring design is shown in Fig. 49. Notice that there are several insertions which contain wigglers. In order to obtain the high repetition rate, we increased the damping rate with the addition of wigglers in straight sections.

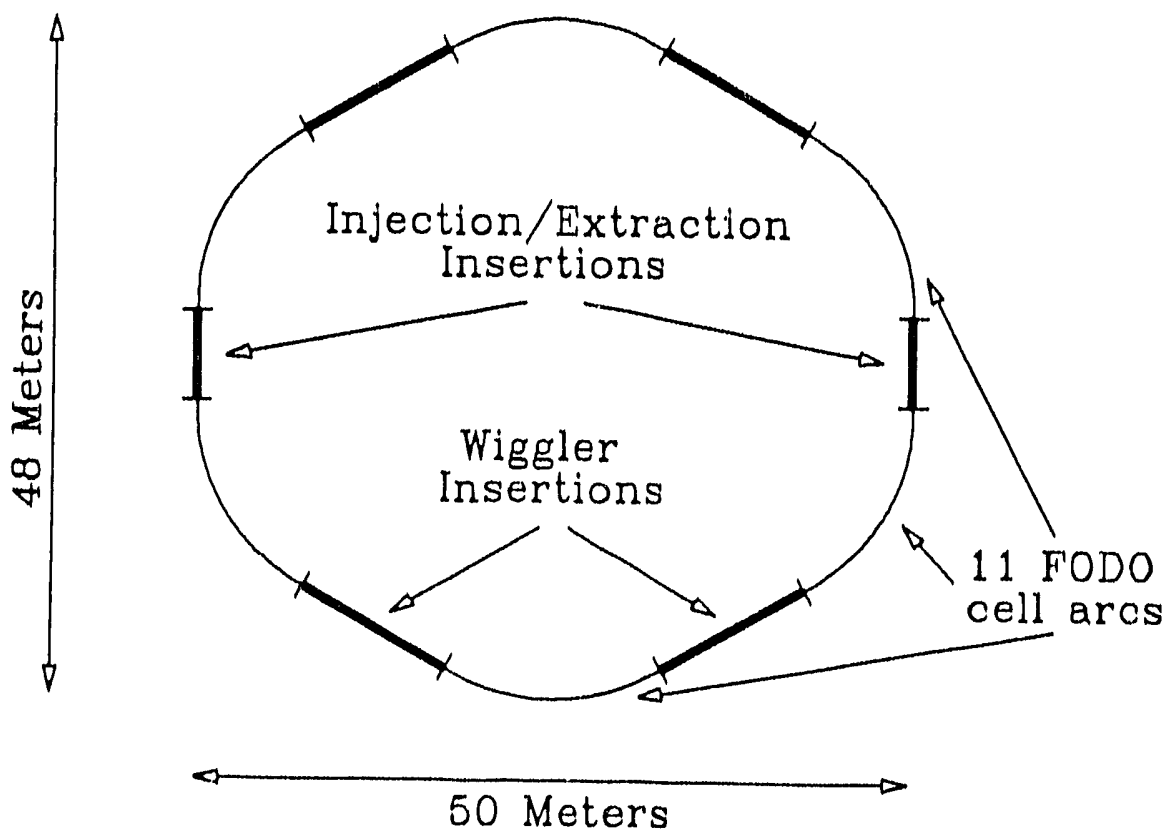

Fig. 49. Schenatic of the NLC damping ring

The basic parameters for the ring are listed in Tables 18, 19, and 20. The lattice is a FODO lattice with combined function bends which change the damping 
partitions, increasing the hordzontal clamping rate at the expense of longitudinal damping. The RF frequency is $1.4 \mathrm{GHz}$ since the bunch spacing in this example is about $20 \mathrm{~cm}$. To meet the repetition ra a requirements, ten batches of bunches must be damped at the same time. Unfortunately, this leaves only 45 ns between the batches for the injection/extraction kickers to turn on or off.

Table 18. NLC damping ring parameters.

\begin{tabular}{|c|c|}
\hline Energy & $E_{0}=1.8 \mathrm{GeV}$ \\
\hline Length & $L=155.1$ meters \\
\hline Lattice & $\begin{array}{c}\text { FODO with } \mathrm{CF} \text { bends and } \\
22 \text { meters of wiggler }\end{array}$ \\
\hline Tunes & $\nu_{x}=24.37, \quad \nu_{y}=11.27$ \\
\hline Momentum compaction & $\alpha=0.00120$ \\
\hline $\begin{array}{c}\text { Design } \\
\text { Current }\end{array}$ & $\begin{array}{c}10 \text { batches of } 10 \text { bunches } \\
\text { of } 2 \times 10^{10} e^{+} / e^{-}\end{array}$ \\
\hline
\end{tabular}

Tables 19 and 20 list the transverse and longitudinal ring parameters when the damping wigglers are both on and off. The wigglers do not strongly effect the emittances of the ring; they are primarily used to clecrease the damping times. Thus, the ring can operate without the wigglers at a reduced repetition rate.

Table 20. NLC damping ring longitudinal parameters.

\begin{tabular}{|c|c|c|}
\hline & Wigglers Off & Wigglers On \\
\hline Radiation/turn, $U_{0}$ & $203 \mathrm{LeV}$ & $468 \mathrm{KeV}$ \\
\hline Energy spread, $\sigma_{c}$ & 0.00128 & 0.00104 \\
\hline Bunch length, $\sigma_{z}$ & $5.1 \mathrm{~mm}$ & $4.6 \mathrm{~mm}$ \\
\hline RF Frequency & $1.4 \mathrm{GHz}$ & $1.4 \mathrm{GHz}$ \\
\hline RF Voltage & $.75 \mathrm{MV}$ & $.75 \mathrm{MV}$ \\
\hline Synch, tunne, $\nu_{s}$ & 0.0075 & 0.0068 \\
\hline$(Z / n)_{\mathrm{cff}}$ & $0.32 \Omega$ & $0.20 \Omega$ \\
\hline
\end{tabular}


Table 19. NLC damping ring transverse parameters.

\begin{tabular}{|c|c|c|}
\hline & Wigglers Off & Wigglers On \\
\hline Natural $\gamma \epsilon_{x}$ & $2.46 \mu \mathrm{mrad}$ & $2.00 \mu \mathrm{mrad}$ \\
\hline$\gamma \epsilon_{x}$ at design current & $3.33 \mu \mathrm{mrad}$ & $2.74 \mu \mathrm{mrad}$ \\
\hline Damping, $\tau_{x}$ & $3.88 \mathrm{~ms}$ & $2.50 \mathrm{~ms}$ \\
\hline Damping, $\tau_{y}$ & $9.19 \mathrm{~ms}$ & $3.98 \mathrm{~ms}$ \\
\hline Damp, partition, $\mathcal{J}_{x}$ & 2.37 & 1.59 \\
\hline Rep, rate, $f_{\mathrm{rep}}$ & $155 \mathrm{~Hz}$ & $360 \mathrm{~Hz}$ \\
\hline Extracted $\gamma \epsilon_{x}$ & $3.33 \mu \mathrm{mrad}$ & $2.74 \mu \mathrm{mrad}$ \\
\hline Extracted $\gamma \epsilon_{y}$ & $0.035 \mu \mathrm{mrad}$ & $0.029 \mu \mathrm{mrad}$ \\
\hline Natural chrom, $\xi_{x}$ & -28.35 & -28.07 \\
\hline Natural chrom., $\xi_{y}$ & -25.10 & -22.27 \\
\hline
\end{tabular}

The threshold impedance $(Z / n)_{\text {eff }}$ is that for the microwave instability at the design current. It is quite small due to the small momentum compaction factor, but is only about a factor of $4-5$ below that obtained in the SLC clamping rings.

The magnet parameters are listed in Table 21. The bending magnets have a length of $20 \mathrm{~cm}$ and a bending field of $13.1 \mathrm{KG}$ with a horizontally defocusing gradient of $300 \mathrm{kG} / \mathrm{m}$. Preliminary POISSON calculations indicate that the gradient, and bending field are achievable, but the short length of the magnets may make end-effects a serious difficulty. ${ }^{(38)}$

For this reason we have re-designed the basic cell. The original cell was a FODO cell with small defocusing quadrupoles; much of the vertical focusing was done in the bends. In the new cell, the defocusing quadrupole is removed and the two bends are joined. Thus, the bending magnet now is $40 \mathrm{~cm}$ long - a more reasonable length. In addition, we elongated the cell and inserted two extra small quadrupoles. These (optional) additional quadrupoles provide another degree of freedom for control of the tunes. The parameters of the two cells are compared in Table 22.

For our design, we chose a wiggler with a peak field of $24 \mathrm{KG}$, a $50 \%$ filling

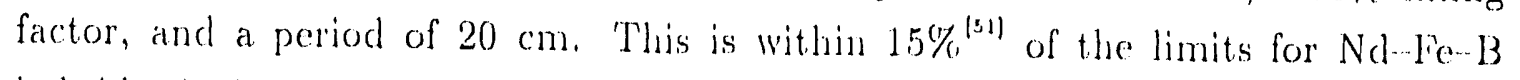
hybrid wigglers as specified in Ref, 50. If such high peak wiggler fields are not possible, the reculued poak field can be dropped to $21 \mathrm{KG}$ by increasing the ring 
Table 21. NLC magnet and wiggler parameters.

\begin{tabular}{|c|c|}
\hline Bends & $\begin{array}{c}B_{0}=13.1 \mathrm{KG} \\
B_{1}=300 \mathrm{KG} / \mathrm{m}\end{array}$ \\
\hline Quadrupoles & $\begin{array}{c}B_{1} \leq 942 \mathrm{KG} / \mathrm{m} \\
\text { pole tip field } \leq 11.3 \mathrm{KG}\end{array}$ \\
\hline Sextupoles & $\begin{array}{c}\text { SF } \int B_{2} d s=-3120 \mathrm{KG} / \mathrm{m}^{2} \\
\text { SD } \int B_{2} d s=4125 \mathrm{KG} / \mathrm{m}^{2}\end{array}$ \\
\hline Wigglers & Length $L_{\text {total }}=22 \mathrm{~m}$ \\
& $\begin{array}{c}\text { Period } \lambda=20 \mathrm{~cm} \\
\text { Gap } g=2 \mathrm{~cm}\end{array}$ \\
& Field $B_{\text {peak }}=24 \mathrm{KG}$ \\
\hline
\end{tabular}

Table 22. Comparison of basic cells.

\begin{tabular}{|c|c|c|c|c|}
\hline & $\gamma \epsilon_{x}$ & $J_{x}$ & $L_{\text {cell }}$ & $\alpha_{m}$ for ring \\
\hline Original FODO & $2.5 \times 10^{-6} \mathrm{~m}-\mathrm{rad}$ & 2.4 & 1.2 & $1.2 \times 10^{-3}$ \\
\hline Modified & $2.5 \times 10^{-6} \mathrm{~m}-\mathrm{rad}$ & 1.9 & 1.5 & $0.8 \times 10^{-3}$ \\
\hline
\end{tabular}

energy to $1.9 \mathrm{GeV}$. Although it would be possible to achieve much higher peak fields with superconducting wigglers, it appears that the non-linear effects increase with the cube of the magnetic field strength. (01)

Finally, the chromaticity is corrected with only two families of sextupoles. The sextupoles are assumed to be constructed with permanent magnets and have lengths of $4 \mathrm{~cm}$, keeping the fields similar to the SLC damping ring sextupoles. After chromatic correction, the dynamic aperture of the perfect machine is just outside the beam pipe. Errors and wigglen non-linearities will reduce the aperture, but hopefully, it can be recovered with a more sophisticated chromaticity correction scheme. Obviously, extensive tracking is needed to determine the dynamic aperture with errors and detailed comparisons with other lattices should be performed to search for superior dynamic aperture characteristics. 


\section{B.3 Future Modifications}

The primary modifications to the ring arise from changes in the desired parameters: first, the desired repetition rate has decreased a factor of two to $180 \mathrm{~Hz}$ because of limitations on the position target, and second, more realistic initial emittances should be used: $\gamma \epsilon_{e^{-}} \sim 3 \times 10^{-5} \mathrm{~m}-\mathrm{rad}$ and $\gamma \epsilon_{e^{+}} \sim 10^{-2} \mathrm{~m}$-rad. The electron initial emittance is comparable to that achieved in the current generation of electron injectors while the increase in the initial positron emittance was suggested to ease the requirements on the positron creation system. ${ }^{[20]}$

The current design without the wigglers will easily achieve the necessary damping for the electron beam. In fact, one might want to decrease the damping rate by decreasing the main bending field while decreasing the number of cells to keep the emittance constant; this will reduce the size, cost, and complexity of the design. But, the wigglers will be needed if a single ring is to achieve the positron requirements. The alternate, and more desirable solution, is to use a pre-damping ring to rapidly damp the positrons to an emittance comparable to that of the initial electron emittance. Then, both the electron and positron main damping rings would be similar. This is the solution currently being employed in the JLC design. ${ }^{[19]}$

\section{B.4 Limitations}

The limitations on achieving the vertical emittance in the damping rings is the subject of Section 11 of this dissertation. Here, we will briefly list a few of the other potential problems that will likely be encountered when designing these future rings.

Dynamic Aperture

To achieve the required horizontal enittances, the damping rings have strong focusing and strong sextupoles to correct the chromaticity. Unfortunately, these sextupoles limit the effective aperture of the ring; particles with large amplitudes are lost due to the nonlinear fields. There are two solutions to this problem: (1) design a ring with large dynamic aperture, and (2) reduce the aperture requirements of the incoming beam.

Increasing the dynamic aperture is currently a topic of considerable research. It is an issue in most of the synchrotron light sources as well as many of the larger colliding beam rings. It is suggested in liefs. 22 and 40, that substantial gains in the dynamic aperture can be realized by using a discrete sextupole arrangement rather 
than the distributed arrangement that is common. This technique may prove to be important for these future rings.

While increasing the dynamic aperture is desirable regardless, the second solution also has many attractive features. The large aperture requirement is dictated by the incoming positron beam which has an initial emittance one-to-two orders of magnitude larger than the electron beam. It was suggested that a pre-damping ring could ease some the damping ring requirements. ${ }^{[38,119]}$ This has been incorporated into the JLC design, where it is shown that, in addition to easing the dynamic aperture and the damping requirements, a pre-damping ring also eases the tolerances on the extraction kicker magnet.

$\underline{\text { Single Bunch Instabilities }}$

The single bunch currents in the damping rings are not very large, but because of the small momentum compaction factor and the small synchrotron tunes, single bunch instabilities could present a problem. The longitudinal microwave instability, which is discussed in Appendix A.6, usually has the lowest threshold current. To avoid this benign instability, the rings must have longitudinal impedances $(Z / n)_{\text {eff }} \sim$ $0.2 \Omega-0.5 \Omega$. This is well over a factor of two smaller than that measured in the SLC ring and requires a very careful vacuum chamber design.

\section{Multi-Bunch Instabilities}

In addition to the single bunch instabilities, there are multi-bunch instabilities that may be severe since many of the designs call for many closely spaced bunches. For example, there are ten batches of ten bunches in the NLC design. The batches are separated by roughly $50 \mathrm{~ns}$ but the bunches within a batch are only separated by about $1 \mathrm{~ns}$. This has been investigated ${ }^{[1 \mathrm{e}]}$ and it was determined that the NLC ring will need specially designed RF cavities. In addition, the ring will likely need feedback systems to further control the instability.

\section{$\underline{\text { RF System }}$}

In addition to multi-bunch instabilities, the NLC RF system will need to handle five times more current than in the SLC rings. This requires careful attention to the higher-order modes. Furthermore, when the bunches are extracted from and injected into the ring there will be large beam loading transients that need to be carefully considered. 
Fast Kicker Magnets

Fast kicker magnets are used to extract or inject the batches of bunches into the damping ring. The kickers need to have very fast rise and fall times so that they do not affect the other stored batches of bunches. The SLC damping rings use a 50 ns bunch separation, but the kickers are a source of problems. Currently, the JLC design ${ }^{(119)}$ assumes a 70 ns batch spacing while the NLC and CLIC designs ${ }^{[30,80]}$ call for a $50 \mathrm{~ns}$ and $22 \mathrm{~ns}$ batch separation, respectively.

The kickers need to have flat pulses so that all bunches within a batch are injected/extracted on the same trajectory. The jitter and flatness tolerances on the extraction kicker are related to the kick needed to extract the beam, typically $25 \mathrm{mrad}$, compared to the beam divergence $\sigma_{x^{\prime}} \sim 20 \mu \mathrm{rad}$. This implies a tolerance of roughly $\Delta \theta / \theta \lesssim 4 \times 10^{-4}$ for a jitter of one tenth the beam size. Such a tolerance will be difficult to achieve.

One suggested solution of easing this tolerance is to use two kickers, driven from the same pulsed power supply, that are separated by $180^{\circ}$ in betatron phase. The first kicker would reside in the ring while the second kicker would be in the extraction line; the second kicker applies exactly the same kick, including jitter, to the beam, removing the jitter from the first kicker.

Synchrotron Radiation Power

The damping rings operate by radiating extensive amounts of beam power. This radiation has three effects: (1) it heats the vacuum chamber, (2) it can damage the magnets and electronic equipment, and (3) it frees gas molecules causing a "dynamic" background gas pressure which can increase the vertical emittance as discussed in Section 2.3.6. All these problems are exacerbated at the damping wigglers, which generate copious quantities of photons in a small region. 


\section{Appendix C \\ COUPLING DERIVATIONS}

In this appendix, we will discuss two issues related to coupling of the emittance; both are relevant to Section 2.2 of this dissertation. In the first section, we discuss the independence of the various contributions to the vertical emittance and then, in the next section, we explicitly perform the algebra needed to derive some the coupling formulas.

\section{C.1 Independence of Contributions to $\epsilon_{y}$}

In Section 2.2.2, we state that the contributions from the opening angle of the radiation, the vertical dispersion, and the betatron and synchro-betatron coupling are all independent and thus they simple add to the emittance/beam size: $\left\langle y_{\beta}^{2}\right\rangle / \beta_{y}$. First, we will consider only contributions to the emittance, and then we will discuss the contributions to the beam size.

There are three contributions to the vertical emittance: the opening angle of the radiation, the vertical dispersion, and the betatron coupling; as discussed in Section 2.2.6, the direct synchro-betatron coupling has a very small contribution to the emittance. These effects increase the vertical emittance by causing random changes in the betatron motion. These changes can be written

$$
\begin{aligned}
& y_{\beta}=y_{\beta 0}+\eta_{y} u / E_{0}+c \eta_{x} u / E_{0}+c^{\prime} \eta_{x}^{\prime} u / E_{0} \\
& y_{\beta}^{\prime}=y_{\beta 0}^{\prime}+\theta_{y} \eta_{y}^{\prime} u / E_{0}+d \eta_{x} u / E_{0}+d^{\prime} \eta_{x}^{\prime} u / E_{0}
\end{aligned}
$$

where $\theta_{y}$ is the opening angle of the radiation and the coefficients $c, c^{\prime}, d$, and $d^{\prime}$ represent the rotation of the eigenvectors due to the betatron coupling; these are functions of the coupling coefficients $Q_{ \pm}$.

Thus, these contributions will add independently to the emittance if

$$
\left\langle u \theta_{y}\right\rangle=\eta_{x} \eta_{y} c\left\langle u^{2}\right\rangle=\eta_{x} \eta_{y}^{\prime} c\left\langle u^{2}\right\rangle=\eta_{x}^{\prime} \eta_{y} c^{\prime}\left\langle u^{2}\right\rangle=\eta_{x}^{\prime} \eta_{y}^{\prime} c^{\prime}\left\langle u^{2}\right\rangle=0
$$

we have assumed that all of the effects are independent of the initial position and angle $\left(y_{\beta 0}, y_{\beta 0}^{\prime}\right)$. The first term is zero since the expected angle of the radiation is zero, but the other terms are not as obvious since the expected value of $\left\langle u^{2}\right\rangle$ is not zero. 
In this case, we have to consider the average of $\eta_{x} \eta_{y} c$ through all the bending magnets; the emittance would depend upon this average. In general, $\eta_{x}$ has a periodicity determined by the lattice cells. Thus, $\eta_{x}$ is constant from cell to cell. In contrast, $\eta_{y}$ and the coupling have periodicities determined by the tunes. The vertical dispersion oscillates at the vertical tune (or the integral portion) while the coupling oscillates at $\nu_{x}+\nu_{y}$ and $\nu_{x}-\nu_{y}$. Provided that $\nu_{x} \neq 2 \nu_{y}$ and $\nu_{y} \neq 2 \nu_{x}$, these averages will be zero. Thus, it is valid to simply add these contributions.

\section{C.2 Derivation of EQS. (2.2.65) AND (2.2.107)}

In this section, we will derive Eqs. (2.2.65) and (2.2.107) from Eq. (2.2.64). We will only explicitly calculate the contributions from the individual coupling resonances; these come from the $\cos \left(\psi_{x}(z)-\psi_{x}\left(z^{\prime}\right)\right) \cos \left(\psi_{y}(z)-\psi_{y}\left(z^{\prime}\right)\right)$ term which is found when one expands the trigonometric functions in Eq. (2.2.64). The derivation of the cross term is similar except one needs to include all of the trigonometric functions; this is easily accomplished using exponential notation, but, because of the large number of terms, the calculation is quite tedious.

First, we expand the square of the bracket in Eq. (2.2.64), keeping only the terms that depend upon the differences of the phases $\psi(z)$ and $\psi\left(z^{\prime}\right)$ :

$$
\frac{\mathcal{N}\left(u^{2}\right\rangle \mathcal{H}_{x}\left(z_{i}\right)}{4 E_{0}^{2}} \iint_{z_{1}}^{s} d z d z^{\prime} \hat{k}(z) \tilde{k}_{(}\left(z^{\prime}\right) \cdots \cos \left(\psi_{y}^{\prime}(z)-\psi_{y}\left(z^{\prime}\right)\right) \cos \left(\psi_{x}(z)-\psi_{x}\left(z^{\prime}\right)\right)
$$

where trigonometric identities have been used in the expansion. Now, we can use additional trigonometric identities to express this result as

$$
\begin{array}{r}
\frac{\sigma_{y}^{2}(s)}{\beta_{y}}=\int_{-\infty}^{s} d z_{i} \frac{\mathcal{N}\left\langle u^{2}\right\rangle \mathcal{H}_{x}}{\delta c E_{0}^{2}} \sum_{ \pm}\left[\left(\int_{z_{1}}^{s} d z e^{\left(z_{1}-z\right) \alpha_{x} / c+(z-s) \alpha_{y} / c} \sqrt{\beta_{x} \beta_{y}} \tilde{k} \cdot \cos \left(\psi_{x} \pm \psi_{x}\right)\right)^{2}\right. \\
\left.+\left(\int_{z_{i}}^{s} d z e^{\left(z_{i}-z\right) \alpha_{x} / c+(z-s) \alpha_{y} / c} \sqrt{\beta_{x} \beta_{y}} \tilde{k} \sin \left(\psi_{x} \pm \psi_{x}\right)\right)^{2}\right]
\end{array}
$$

where the sum over \pm represents a sum over the $\psi_{x}+\psi_{y}$ phase and the $\psi_{x}-\psi_{y}$ phase.

Next, we can condense this into a single integral using complex exponentials and we separate the integral over $z$ into a portion over an integral number of turns of 
the ring and a portion over the remaining segment. Thus, the result has the form:

$$
\int_{-\infty}^{s} d z_{i}\left[\int_{z_{i}}^{s} d z \cdots\right]^{2} \Rightarrow \sum_{n}^{\infty} \int_{s-C}^{s} d z_{i}\left[\sum_{j}^{n-1} \int_{s-C}^{s} d z \cdots+\int_{z_{i}-n C}^{s-n C} d z \cdots\right]^{2}
$$

where $\cdots$ is used to represent the integrand. Explicitly, this yields

$$
\begin{aligned}
\frac{\sigma_{y}^{2}(s)}{\beta_{y}}=\sum_{n=0}^{\infty} \int_{s-C}^{s} d z_{i} \frac{\mathcal{N}\left\langle u^{2}\right\rangle \mathcal{H}_{x}\left(z_{i}\right)}{8 c E_{0}^{2}} \sum_{ \pm} \mid e^{-n \alpha_{x} T_{0}} \sum_{j=0}^{n-1} e^{j\left(\alpha_{x}-\alpha_{y}\right) T_{0}-i j 2 \pi \Delta \nu_{ \pm}} \int_{s-C}^{s} q_{ \pm}(z) d z \\
+\left.e^{-n \alpha_{y} T_{0}-i n 2 \pi \Delta \nu_{ \pm}} \int_{z_{i}}^{s} q_{ \pm}(z) d z\right|^{2}
\end{aligned}
$$

where

$$
q_{ \pm}(z)=\sqrt{\beta_{x} \beta_{y}} \tilde{k} e^{i\left(\psi_{x} \pm \psi_{x}\right)} .
$$

In addition, $\Delta \nu_{ \pm}=\left(\nu_{x} \pm \nu_{y}\right), T_{0}$ is the revolution time, and it was assumed that the damping per revolution is small compared to the betatron tunes.

Now, we perform the sum over $j$. The expression within the absolute value signs becomes

$$
\begin{gathered}
\mid \frac{i e^{i \pi \Delta \nu_{ \pm}}}{2 \sin \pi \Delta \nu_{ \pm}}\left(e^{-n \alpha_{y} T_{0}-i n 2 \pi \Delta \nu_{ \pm}}-e^{-n \alpha_{x} T_{0}}\right) \int_{s-C}^{s} q_{ \pm}(z) d z \\
+\left.e^{-n \alpha_{y} T_{0}-i n 2 \pi \Delta \nu_{ \pm}} \int_{z_{1}}^{s} q_{ \pm}(z) d z\right|^{2}
\end{gathered}
$$

At this point, we can calculate the case where the local coupling is zero at location s. When the local coupling is zero, the first integral over $q$ is zero and we are left with only the second term. Thus,

$$
\frac{\sigma_{y}^{2}(s)_{\text {global }}}{\beta_{y}}=\sum_{n=0}^{\infty} e^{-2 n \alpha_{y} T_{0}} \int_{s-C}^{s} d z_{i} \frac{\mathcal{N}\left\langle u^{2}\right\rangle \mathcal{H}_{x}\left(z_{i}\right)}{8 c E_{0}^{2}} \sum_{ \pm}\left|\int_{z_{1}}^{s} q_{ \pm}(z) d z\right|^{2}
$$

Now, we perform the final summation, shifting $s \rightarrow s+C$, and assuming that the photons are radiated uniformly around the ring, this yields E(1. (2.2.107). 
If the local coupling is not zero, we can group the terms in Eq. (C.2.6) as

$$
\begin{aligned}
& \mid-e^{-n \alpha_{s} T_{0}}\left(\frac{i e^{i \pi \Delta \nu_{ \pm}}}{2 \sin \pi \Delta \nu_{ \pm}} \int_{s=C}^{s} q_{ \pm}(z) d z\right) \\
& +\left.e^{-n \alpha_{y} T_{0}-i n 2 \pi \Delta \nu_{ \pm}}\left(\frac{i e^{i \pi \Delta \nu_{ \pm}}}{2 \sin \pi \Delta \nu_{ \pm}} \int_{s-C^{\prime}}^{s} q_{ \pm}(z) d z+\int_{z_{i}}^{s} q_{ \pm}(z) d z\right)\right|^{2} .
\end{aligned}
$$

When the absolute value sign is calculated, the cross term will have an oscillatory term due to the complex exponential. Assuming that $2 \pi \Delta \nu_{ \pm} \gg \alpha T_{0}$, this cross term will go to zero when the final sum over $n$ is performed. Thus, we are left with the separate absolute values of the two terms in Eq. (C.2.8). The first term is simple; the absolute value is

$$
\frac{e^{-2 n \alpha_{x} T_{0}}}{4 \sin ^{2} \pi \Delta \nu_{ \pm}}\left|\int_{g}^{g+C} q_{ \pm}(z) d z\right|^{2}
$$

After performing the final sum over $n$ and substituting with the equilibrium emittance, this yields the first expression in $\mathrm{Eq}$. (2.2.65).

Finally, we have the second term of Eq. (C.2.8). Let us express this as

$$
\frac{e^{-2 n \alpha_{y} T_{0}}}{4 \sin ^{2} \pi \Delta \nu_{ \pm}}\left|\left(i e^{i \pi \Delta \nu_{ \pm}}+2 \sin \pi \Delta \nu_{ \pm}\right) \int_{z_{1}}^{s} q_{ \pm}(z) d z+i e^{i \pi \Delta \nu_{ \pm}} \int_{s-C}^{z_{i}} q_{ \pm}(z) d z\right|^{2}
$$

Next, we express the sine in exponential form and shift the second integral by $C$. When we shift the limits by $C$, we have to include a phase shift of $e^{i 2 \pi \Delta \nu_{ \pm}}$. Thus, Eq. (C.2.10) becomes

$$
\frac{e^{-2 n \alpha_{y} T_{0}}}{4 \sin ^{2} \pi \Delta \nu_{ \pm}}\left|i e^{-i \pi \Delta \nu_{ \pm}} \int_{z_{i}}^{s} q_{ \pm}(z) d z+i e^{-i \pi \Delta \nu_{ \pm}} \int_{s}^{z_{i}+C} q_{ \pm}(z) d z\right|^{2}
$$

Now, we add these two integrals and perform the final summation over $n$; this yields the second expression in Eq. (2.2.65). 


\section{Appendix D \\ NLC LINEAR ACCELERATORS}

\section{D.1 Basic Scaling}

As illustrated in Fig. 1, the proposed NLC is to consist of two linear accelerators: a preliminary linac that accelerates the beam from the damping ring to the final bunch compression, and a main linac that then accelerates the beam to the final energy. The current design calls for the main linac to operate at an RF frequency of $11.4 \mathrm{GHz}$ with a large accelerating gradient. In contrast, the low energy linac would operate at $2.8 \mathrm{GHz}$ with a lower gradient; the low energy linac would be comparable to the SLC linac.

The higher frequency RF is used in the main linac to reduce both the average power consumption and the length of the linac. But, before the second bunch compression, the wakefields in a high frequency linac would rapidly dilute the transverse emittance; the transverse wakefield increases with both the bunch length and the RF frequency. Unfortunately, the second bunch compression can not be performed at low energy since the relative energy spread would then be the order of many percent and the transverse beam emittance would be diluted by the dispersive and chromatic errors.

Both the preliminary and main linacs are assumed to be constructed of FODO cells that are scaled as the beam is accelerated. A basic FODO cell is composed of a focusing quadrupole, a drift, usually filled with accelerator sections, a defocusing quadrupole, and another drift section. An example FODO cell with lattice functions is illustrated in Fig. 50. In the "thin-lense" approximation where the length of the quadrupole magnets is neglected, one can find simple expressions for the beta. functions. ${ }^{[120]}$ In particular, the maximum and minimum beta functions are

$$
\hat{\beta}=L_{c} \frac{1+\sin \psi_{c} / 2}{\sin \psi_{c}} \quad \text { and } \quad \check{\beta}=L_{c_{c}} \frac{1-\sin \psi_{c} / 2}{\sin \psi_{c}^{\prime}}
$$

where $L_{c}$ is the length of the cell and $\psi_{c}$ is the phase advance per cell. Finally, the cell length and the phase advance can be simply related to the integrated quadrupole strengths as

$$
L_{c}=\left|\frac{4}{K_{1} L_{q}}\right| \sin \psi_{c} / 2
$$

where $K_{1}$ is the normalized quadrupole strength and $L_{q}$ is the quadrupole length. 


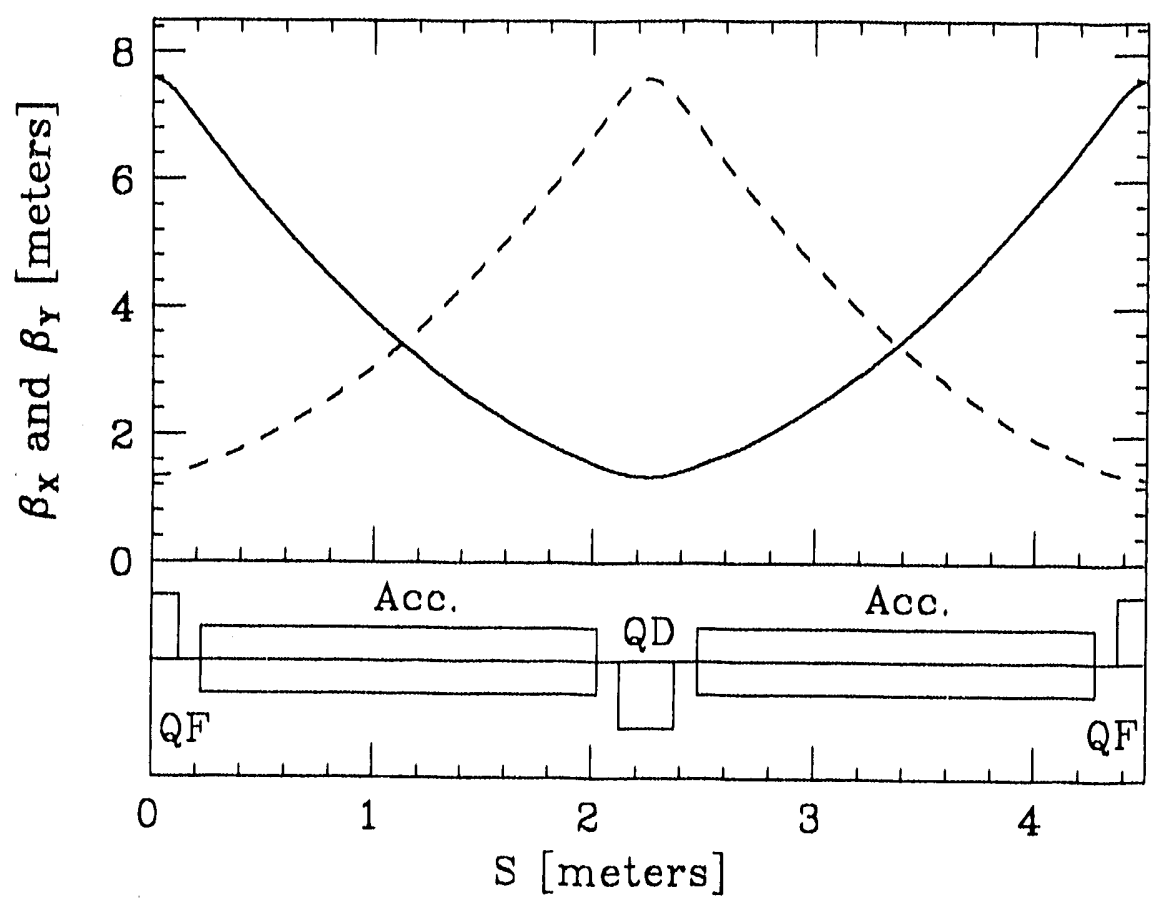

Fig. 50. Lattice functions for a basic FODO cell; the solid and dashed lines are $\beta_{x}$ and $\beta_{y}$, respectively. QF and $\mathrm{QD}$ represent the focusing and defocusing quadrupoles.

Alternately, we can use the beta functions to find the relations

$$
\left|K_{1} L_{q}\right|(\hat{\beta}-\check{\beta})=4 \tan \psi_{c} / 2 \quad \text { and } \quad\left|K_{1} L_{q}\right|(\hat{\beta}+\check{\beta})=\frac{4}{\cos \psi_{c} / 2} .
$$

As the beam is accelerated, the normalized quadrupole gradient $K_{1}$ of a given magnetic field decreases inversely with the beam energy. If the cells are not scaled, the beta function will increase linearl! with energy while the phase advance per cell decreases. Since many of the tolerances depend upon the local value of the beta function, this is not a good choice. Instead, a scaling is typically chosen where the magnetic field strength is kept constant, but the cell lengths and the quadrupole magnet lengths increase with the square root of the beam energy. ${ }^{[101]}$ Such a lattice has beta functions that increase with the square root of the beam energy while the phase advance per cell remains constant. Thus,

$$
\begin{array}{rlrl}
K_{1}=K_{10} \frac{\gamma_{0}}{\gamma} & \beta & =\beta_{0} \sqrt{\frac{\gamma}{\gamma_{0}}} \\
L_{q}=L_{q 0} \sqrt{\frac{\gamma}{\gamma_{0}}} & L_{c}=L_{c 0} \sqrt{\frac{\gamma}{\gamma_{0}}} .
\end{array}
$$

This lattice has the advantage that it makes "optimal" use of the quadrupole 
magnets. Unfortunately, such a scaling will cause extensive complications because every magnet, drift, and accelerator section is different. Instead, one could scale the lattice in a number of small discrete steps to approximate the $\sqrt{\gamma}$ behavior. Regardless, we will adopt this scaling because it simplifies the analytic treatment of the lattice.

At this point, we need to describe how this scaling is used. First, Eq. (D.1.4) gives the basic scaling of the parameters. Furthermore, many of the calculations in Section III of this thesis will involve sums over the quadrupole magnets having the form:

$$
\sum_{n=1}^{N_{q}} f\left(\gamma_{n}\right)
$$

where $N_{q}$ is the number of quadrupoles and $\gamma_{n}$ is the beam energy at the $n$th quadrupole. To evaluate these sums, we will replace them with an integral

$$
\int_{0}^{N_{q}} d n f(\gamma(n))
$$

which is valid provided that $N_{q}$ is large and $\gamma(n)$ varies slowly as a function of $n$. Now, for the scaling derived above, $\gamma(n)$ can be expressed as

$$
\gamma(n)=\left(\frac{n}{c}+\sqrt{\gamma_{0}}\right)^{2}, \quad \text { where } \quad c=\frac{N_{q}}{\sqrt{\gamma_{f}}-\sqrt{\gamma_{0}}} .
$$

This dependence of $\gamma$ on $n$ will give slightly different numerical results than the linear dependence implicitly assumed in Refs. 99, 98, 97, and 53.

Finally, we also need to describe our approximation of the wakefield deflections. As discussed in Section 3.4.2, the dipole wakefield occurs when the beam travels off-axis in an accelerator structure and it deflects the tail of the beam. In the two particle model described in Section 3.4.2, the deflection of the tail particle can be written

$$
\begin{aligned}
& \Delta \theta_{w}(s)=\int_{0}^{s} d s^{\prime} \frac{N r_{0} W_{1 \perp}}{4 \gamma} y_{c}\left(s^{\prime}\right) R_{22}\left(s^{\prime}, s\right) \\
& \Delta y_{w}(s)=\int_{0}^{s} d s^{\prime} \frac{N r_{0} W_{1 \perp}}{4 \gamma} y_{c}\left(s^{\prime}\right) R_{12}\left(s^{\prime}, s\right),
\end{aligned}
$$

where $\Delta \theta_{w}$ and $\Delta y_{w}$ are the change in angle and position due to the wakefield. 
We will approximate this with a delta-function deflection located at the center of the structure where the deflection depends upon the average trajectory offset through the structure. In this approximation, $\Delta \theta_{w}$ is calculated correctly but there is a smaller error in calculating $\Delta y_{w}$. At the end of the structure, $\Delta y_{w}$ should be

$$
\Delta y_{u}=\frac{N r_{0} W_{1 \perp}}{4 \gamma} L_{a c c}\left(\frac{y_{0} L_{a c c}}{2}+\frac{y_{0}^{\prime} L_{a c c}^{2}}{12}\right)
$$

but our approximation neglects the $y_{0}^{\prime}$ dependence; this causes a small error when calculating the effect of a random trajectory.

Table 23. Average beta functions for various cell phase advances.

\begin{tabular}{|c|c|c|}
\hline$\psi_{c}$ & $\bar{\beta}$ & $\bar{\psi}$ \\
\hline $60^{\circ}$ & $L_{c} / 1.01$ & $10.9^{\circ}$ \\
\hline $75^{\circ}$ & $L_{c} / 1.19$ & $12.2^{\circ}$ \\
\hline $90^{\circ}$ & $L_{c} / 1.34$ & $12.8^{\circ}$ \\
\hline $105^{\circ}$ & $L_{c} / 1.41$ & $12.6^{\circ}$ \\
\hline
\end{tabular}

Lastly, we need to know the beta function at the center of the structure. Assuming the structure is centered between the two adjacent quadrupoles, we find

$$
\bar{\beta}=\left(\frac{L_{c}}{4 \sin \bar{\psi}}\right)^{2} \frac{\sin \psi_{c}}{L_{c}\left(1+\sin \psi_{c} / 2\right)}
$$

where $\bar{F}$ is the phase difference between the center of the structure and adjacent focusing quadrupole. This can be written

$$
\tan \bar{\psi}=\frac{\sin \psi_{c}}{4\left(1+\sin \psi_{c}^{\prime} / 2\right)\left(1-\frac{\sin \psi_{c}^{\prime} / 2}{2}\right)} .
$$

Values of $\bar{\beta}$ and $\bar{\psi}$ are listed in Table 23 for various cell phase advances. Notice that the beta function at the center of the cell decreases as the phase advance per cell increases. Also note that $\bar{\beta}$ is slightly different from the average beta: $(\hat{\beta}+\ddot{\beta}) / 2$, although this is a commonly used approximation. 
Table 24. Parameters for the preliminary NLC linac.

\begin{tabular}{|c|c|c|}
\hline & Version 1 & Version 2 \\
\hline Energy & $1.8 \rightarrow 16.5 \mathrm{GeV}$ & $1.8 \rightarrow 16.5 \mathrm{GeV}$ \\
\hline Gradient & $20 \mathrm{MeV} / \mathrm{m}$ & $20 \mathrm{MeV} / \mathrm{m}$ \\
\hline Structure length & $1.5 \mathrm{~m}$ & $3.0 \mathrm{~m}$ \\
\hline \# of acc, sections, $N_{a c c}$ & 500 & 250 \\
\hline Linac length & $1000 \mathrm{~m}$ & $900 \mathrm{~m}$ \\
\hline \# of cells, $N_{c e l l}$ & 125 & 62 \\
\hline$\hat{\beta}_{0}$ & $6.5 \mathrm{~m}$ & $13 \mathrm{~m}$ \\
\hline$\beta_{0}$ & $1.1 \mathrm{~m}$ & $2.2 \mathrm{~m}$ \\
\hline $\bar{\beta}_{0}$ & $2.8 \mathrm{~m}$ & $5.7 \mathrm{~m}$ \\
\hline$\psi_{x}$ per cell & $86^{\circ}$ & $86^{\circ}$ \\
\hline$\psi_{y}$ per cell & $94^{\circ}$ & $94^{\circ}$ \\
\hline Initial cell length & $3.8 \mathrm{~m}$ & $7.6 \mathrm{~m}$ \\
\hline Initial beam size, $\sigma_{y i}$ & $5.5 \mu \mathrm{m}$ & $7.8 \mu \mathrm{m}$ \\
\hline Final beam size, $\sigma_{y f}$ & $3.0 \mu \mathrm{m}$ & $4.3 \mu \mathrm{m}$ \\
\hline Bunch length. $\sigma_{z}$ & $500 \mu \mathrm{m}$ & $500 \mu \mathrm{m}$ \\
\hline
\end{tabular}

\section{D.2 Preliminary LinaC}

The preliminary NLC linac will acclerate the beams after the first bunch compression at $1.8 \mathrm{GeV}$ to the second bunch compression at $16.5 \mathrm{GeV}$. In Table 24, we list parameters for two versions of this linac which differ in the degree of focusing; detailed parameters have not been determined yet.

Both linacs are assumed to be constructed of FODO cells that are scaled with the square root of the beam encrgy as described in Section D.1. In addition, the linacs are assumed to have an RT frequency of $2.8 \mathrm{GHz}$ with accelerating gradients of $20 \mathrm{MeV} / \mathrm{m}$; this is similar to the SLAC accelerator structures. 
Table 25. Parameters for the main NLC linac.

\begin{tabular}{|c|c|c|}
\hline & Low energy & High energy \\
\hline Energy & $16.5 \rightarrow 250 \mathrm{GeV}$ & $16.5 \rightarrow 500 \mathrm{GeV}$ \\
\hline Gradient & $100 \mathrm{MeV} / \mathrm{m}$ & $100 \mathrm{MeV} / \mathrm{m}$ \\
\hline Structure length & $2.3 \mathrm{~m}$ & $1.8 \mathrm{~m}$ \\
\hline \# of acc, sections, $N_{a c c}$ & 1000 & 2700 \\
\hline Linac length & $2900 \mathrm{~m}$ & $5700 \mathrm{~m}$ \\
\hline \# of cells, $N_{\text {cell }}$ & 210 & 380 \\
\hline$\hat{\beta}_{0}$ & $9.5 \mathrm{~m}$ & $7.5 \mathrm{~m}$ \\
\hline$\check{\beta}_{0}$ & $1.6 \mathrm{~m}$ & $1.3 \mathrm{~m}$ \\
\hline $\bar{\beta}_{0}$ & $4.2 \mathrm{~m}$ & $3.3 \mathrm{~m}$ \\
\hline$\psi_{x} /$ cell & $86^{\circ}$ & $86^{\circ}$ \\
\hline$\psi_{y} /$ cell & $94^{\circ}$ & $94^{\circ}$ \\
\hline Initial cell length & $5.6 \mathrm{~m}$ & $4.4 \mathrm{~m}$ \\
\hline Initial beam size, $\sigma_{y i}$ & $2.0 \mu \mathrm{m}$ & $1.7 \mu \mathrm{m}$ \\
\hline Final beam size, $\sigma_{y f}$ & $1.0 \mu \mathrm{m}$ & $0.7 \mu \mathrm{m}$ \\
\hline Bunch length, $\sigma_{z}$ & $100 \mu \mathrm{m}$ & $100 \mu \mathrm{m}$ \\
\hline
\end{tabular}

\section{D.3 Main LinaC}

The main NLC linac will accelerate the beams after the second bunch compression to the final energy. In Table 25, we list parameters of two linacs that are used for discussion in this dissertation. 'These are only illustrative examples; detailed parameters have not been determined.

Both linacs are assumed to be constructed of FODO cells that are scaled with the square root of the beam energy as described in Section D.1. In addition, it is assumed that the linacs have an RF frequency of $11.4 \mathrm{GHz}$ with accelerating gradients of $100 \mathrm{MeV} / \mathrm{m}$. Wakefields for an $11.4 \mathrm{GHz}$ structure with an iris size $a / \lambda_{\mathrm{RF}}=0.20$ are illustrated in Fig. $51^{\{10\}}$ 


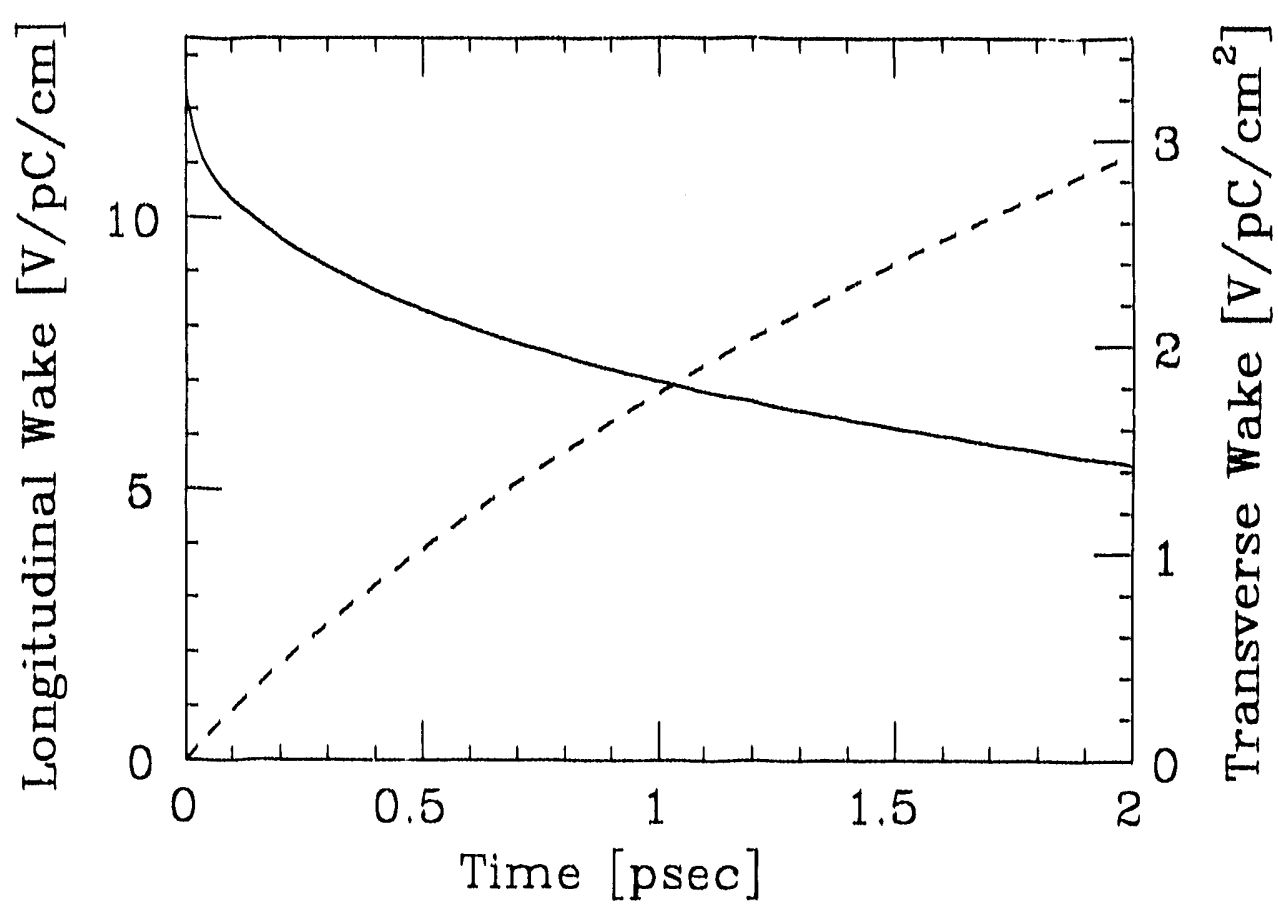

Fig, 51. Longitudinal (solid) and transverse (dashed) wakefields for 11.4 $\mathrm{GHz}$ NLC main linac accelerator structure; the iris radius is $a / \lambda_{\mathrm{RF}}=0.2$. 


\section{REFERENCES}

1. C. Adolphsen, K. Bane, and J. Seeman, "Effect of Wakefields on First Order" Transport in the SLC LINAC," Proc, of the 1991 Part. Acc, Conf., San Francisco, CA (1991); and SLAC-PUB-5581.

2. C. Adolphsen, P. Emma, T. Fieguth, and W. Spence, "Chromatic Correction in the SLC Bunch Length Compressors," Proc, of the 1991 Part. Acc. Conf., San Francisco, CA (1991); and SLAC-PUB-5584.

3. C. Adolphsen, et. al., "Beam-Based Alignment Technique for the SLC Linac," Presented at the IEEE Part. Acc. Conf., Chicago, IL (1989).

4. T. Aniel and J. L. Laclare, "Sensitivity of the ESRP Machine to Ground Movement," ESRF-SITE-086-04 (1986).

5. R. Baartman and U. Wienands, "Synchro-Betatron Resonances in the Presence of Tune Modulation," Proc. of the 2nd European Part. Acc. Conf., Nice, France (1990).

6. Y. Baconnier, "Ncutralization of Accelerator Beams by Ionization of the Residual Gas," Proc, of the CERN General Accelerator Physics Course, CERN 85-19, p. 267 (1985).

7. P. P. Bagley and D. L. Rubin, "Correction of 'Transverse Coupling in a Storage Ring," Proc. of the 1989 IEEE Part. Acc. Conf., Chicago IL. (1989); and P. P. Bagley, Ph.D. Thesis, Cornell University, in preparation.

8. V. Balakin, A. Novokhatsky, V. Smirnov, "VLEPP: Transverse Beam Dynamics," Proceedings of the 12th Int. Conf. on High Energy Accelerators, Fermilab, (1983).

9. K. Bane, P. Wilson, and T. Weiland, "Wakefields and Wakefield Acceleration," AIP Conf. Proc. 127: Proc, of the 1983 US Part. Acc. School, AIP, New York (1984); and SLAC-PUB-352S.

10. K. Bane, private communication.

11. D. Barber, H. Mais, G. Riplien, F. Willeke, "Nonlinear Theory of Coupled Synchro-Betatron Motion," DESY 86-147 (1986).

12. M. Bassetti and G. A. Erskine, "Closed Expression for the Electric Field of a Two-dimensional Gaussian Charge," CERN ISR-TH/80-16 (1980). 
13. M. Bassetıi, S. Guiducci, L. Palumbo, "General Considerations on a Damping Ring for a 5-10 GeV Linear Collider," Proc. of the 1st European Part. Acc. Conf., Rome, Italy (1988).

14. C. Bender and S. Orszag, Advanced Mathematical Mcthods for Scientists and Engineers, McGraw-Hill Inc., USA (1978).

15. J. Bengtsson, "Non-Linear Transverse Dynamics for Storage Rings with Applications to the Low-Energy Antiproton Ring (LEAR) at CERN," CERN 88-05, Ph.D. Thesis (1988).

16. H. Bethe and J.Ashkin, "Passage of Radiations through Matter," Experimental Nuclear Physics, Vol. 1, edited: E. Segre, Wiley, New York (1953).

17. J. D. Bjorken and S. K. Mtingwa, "Intrabeam Scattering," Particle Accelerator, 13, 115 (1983).

18. C. J. Bocchetta and A. Wrulich, "The Trapping and Clearing of Ions in ELETTRA," Nucl. Inst. and Meth., A278, 807 (1989).

19. I. Borchardt, E. Karantzoulis, H. Mais, G. Ripken, "Calculation of Transverse and Longitudinal Space Charge Effects within the Framework of the Fully Six-Dimensional Formalism," Z. Phys., C41, 25 (1988).

20. H. Braun, private communication.

21. D. Briggs, et. al., "Computer Modeling of Bunch-by-Bunch Feedback for the SLAC B-Factory Design," Proc. of the 1991 Part. Acc. Conf., San Francisco, CA (1991); and SLAC-PUB-5466.

22. R. Brinkmann, "A Study of Low Emittance Damping Ring Lattices," DESYM-90-09 (1990).

23. K. L. Brown and R. Servranckx, "First and Second Order Charged Particle Optics," AIP Conf. Proc. 127: Proc, of the 1983 US Part. Acc. School, AIP, New York (1984); and SLAC-PUB-3381.

24. H. Bruck, "Circular Particle Accelerators: translation," LA-TR-72-10 (1972).

25. F. Bulos, private communication.

26. A. W. Chao and M. J. Lee, "Particle Distribution Parameters in an Flectron Storage Ring," J. App. Phys., 47, 4453 (1976).

27. A. W. Chao and M. J. Lee, "Vertical Beam Size due to Orbit and Alignment Errors," SLAC-PUB-1915 (1977). 
28. A. W. Chao, "Evaluation of Beam Distribution Parameters in an Electron Storage Ring," J. Appl. Phy., 50, 595 (1979).

29. A. W. Chao, "Coherent Instabilities of a Relativistic Bunched Beam," AIP Conf. Proc. 105: Proc. of the 1982 US Part. Acc. School, AIP, New York (1982); and SLAC-PUB-2946.

30. A. W. Chao and R. K. Cooper, "Transverse Quadrupole Wakefield Effects in High Intensity Linacs," Particle Accelerators, 13, 1 (1983).

31. A. W. Chao, B. Richter, and C. Y. Yao, "Beam Emittance Growth Caused by Transverse Deflecting Fields in a Linear Accelerator," Nucl. Instr. Meth., 178, 1 (1980).

32. Pisin Chen, "Disruption, Beamstrahlung, and Beamstrahlung Pair Creation," Proceedings of the DPF Summer Study, Snowmass '88, Snowmass, CO (1988).

33. E. Close, et. al., "A Proposed Orbit and Vertical Dispersion Correction System for PEP," SLAC-PEP-271 (1978).

34. E.D. Courant and H.S. Snyder, "Theory of the Alternating-Gradient Synchrotron," Annals of Phys., 3, 1 (1958).

35. F. J. Decker, et. al., "Dispersion and Betatron Matching into the Linac," Proc. of the 1991 Part. Acc. Conf., San Francisco, CA (1991); and SLACPUB-5484.

36. J. P. Delahaye and J. P. Potier, "Reverse Bending Magnets in a CombinedFunction Lattice for the CLIC Damping Ring," Proc. of the 1989 IEEE Part. Acc. Conf., Chicago, IL (1989).

37. J. P. Delahaye and J. P. Potier, "Study of Lattices Optimized for Small Emittances and Strong Damping," CERN CLIC Note 130 (1991).

38. R. A. Early, private communication.

39. D. A. Edwards, "Oscillation Damping Factors for Off-Momentum Orbits in Electron Storage Rings," FERMILAB-TM-0.566 (1975).

40. L. Emery, "A Wiggler-Based Ultra-Low Emittance Damping Ring Lattice and Its Chromatic Correction," Ph.D. Thesis, Stanford University, (1990).

41. M. Enert, et. al., "Transverse and Longitudinal Multi-Bunch Feedback Sy'stems for PETRA," DESY'-91-036 (1991). 
42. G. E. Fischer, "Ground Motion and it's Effect in Accelerator Design," AIP Conf. Proc, 153: Proc. of the 1985 US Part. Acc. School, AIP, New York (1987).

43. G. E. Fischer, private communication.

44. I. S. Gradshteyn and 1. M. Ryzhik, Tables of Integrals, Series, and Products, Academic Press, Orlando FL (1980). Integral 6.576.4.

45. I. M. Gradshteyn and I. M. Ryzhik, ibid. Integral 3.251.5.

46. G. Guignard, "The General Theory of All Sum and Difference Resonances in a Three-Dimensional Magnetic Field in a Synchrotron: Parts I and II," CERN ISR-MA/75-23 and CERN ISR-MA/75-35 (1975).

47. G. Guignard, "Linear Coupling in Storage Rings with Radiating Particles," CERN ISR-BOM/79-30 (1979).

48. G. Guignard, "Betatron Coupling with Radiation," Proc. of the 1985 CERN Advanced Acc. School, CERN 87-03 (1987), p. 203.

49. J. Haissinski, Ph.D. Thesis, Orsay (1965).

50. K. Halbach, J. Appl. Phys, 57, 3605 (1985).

51. K. Halbach, private communication.

52. D. Heins, et. al., "Wide Band Multi-Bunch Feedback Systems for PETRA," DESY 89-157 (1989).

53. H. Henke, "Transport and Acceleration of Lo:v-Emittance Electron Beams," Proc. of the 1988 Linear Acc. Conf., Williamsburg, VA (1988).

54. R. O. Hettel, "Beam Steering and Stabilization Systems: Present Status and Considerations for the Future," Nucl. Meth. and Instr., A266, 155 (1988).

55. T. Himel, L. Hendrickson, F. Rouse, and H. Shoaee, "Use of Digital Control Theory State Space Formalism for Feedback at SLC," Proc. of the 1991 Part. Acc. Conf., San Francisco, CA (1991); and SLAC-PUB-5470.

56. K. Hirata, K. Oide, and B. Zot.ter, "Synchrotron Radiation Limit on the Luminosity in TeV Linear Colliders," Phys, Let., B244, 437 (1989).

57. N. Holtkamp, private communication.

58. A. Hutton, International Workshop on the Next Generation Linear Colliders, SLAC-335 (1!)88). 
59. H. Ikegami, "Cyclotron Maser Cooling of Electron and Ion Beams," Phys. Rev. Let., 64, 1737 (1990).

60. J. D. Jackson, Classical Electrodynamics, Wiley, New York (1975).

61. C. Johnson, "Beam Neutralization and its Effects in Accelerator Storage Rings," Ph.D. Thesis, Univ. Wisconsin-Madison (1989).

62. J. M. Jowett, "Introductory Statistical Mechanics for Electron Storage Rings," AIP Conf. Proc. 153: Proc. of the 1985 US Part. Acc. School, AIP, New York (1987).

63. T. Katsura, et. al., "A Beam Position Feedback System for Beam Lines at the Photon Factory," Proc. of the 1987 IEEE Part. Acc. Conf., Washington, D.C. (1987).

64. J. Kewisch, T. Limberg, J. Rossbach, F. Willeke, "Vertical Dispersion Generated by Correlated Closed Orbit Deviations," DESY 86-020 (1986).

65. Landau and Lifshitz, Non-Relativistic Quantum Mechanics, Pergamon Press, Oxford (1965).

66. L. J. Laslett, "On Intensity Limitations Imposed by Transverse Scape Charge Effects in Circular Particle Accelerators," Proc. of 1963 Summer Study on Storage Rings, BNL Report 7534 (1963).

67. T. L. Lavine, et. al., "Beam Determination of the Quadrupole Misalignments and Beam Position Monitor Biases in the SLC Linac," Presented at the 1988 Linear Acc. Conf., Williamsburg, VA (1988).

68. J. LeDuff, "Current and Density Limitations in Existing Electron Storage Rings," Nucl. Instr, and Meth., A239, 83 (1985).

69. G. Leleux, P. Nghiem, and A. Tkatchenko, "Synchrotron Radiation Perturbations in Long Transport Lines," Proc. of the 1991 Part. Acc. Conf., San francisco, CA (1991); and LNS/GT 91-04.

70. T. Limberg, J. Seeman, and W. Spence, "Effects and Tolerances of Injection Jitter in the SLC and Future Linear Colliders," Proc. 1990 European Part. Acc. Conf., Nice, France (1990).

71. G. Lüders, Nuovo Cimento Suppl., 2, 1075 (1955).

72. M. Martini, CERN PS/84-9 (AA) (19S4). 
73. N. Merminga, P. L. Morton, J. T. Seeman, W. L. Spence, "Transverse Phase Space in the Presence of Dispersion," Proc. of 1991 Part. Acc. Conf., San Francisco, CA (1991); and SLAC-PUB-5514.

74. N. Merminga, J. Irwin, R. Helm, and R. D. Ruth, "Optimizing a Nonlinear Coilimation System for Future Linear Colliders," Proc. of 1991 Part. Acc. Conf., San Francisco, CA (1991); and SLAC-PUB-5507.

75. B. W. Montague, "Fourth-Order Coupling Resonance Excited by Space Charge Forces in a Synchrotron," CERN 68-38 (1968).

76. R. J. Nawrocky, et. al., "Automatic Beam Streering in the NSLS Storage Rings Using Closed Orbit Feedback," Nucl. Meth. and Instr., A266, 164 (1988).

77. K. Oide, "Synchrotron Radiation Limit on the Focussing of Electron Beams," Phys. Rev. Let., 61, 1713 (1988).

78. R. B. Palmer, "The Interdependence of Parameters for the TeV Linear Collider," SLAC-PUB-4295 (1987).

79. A. Piwinski, "Intrabeam Scattering," Proc. 9th Int. Conf. on High Energy Accelerators, Stanford, CA (1974).

80. A. Piwinski and A. Wrulich, "Excitation of Betatron-Synchrotron Resonances by a Dispersion in the Cavities," DESY 76/07 (1976).

81. A. Piwinski, "Intrabeam Scattering with Vertical Dispersion," Proc. of the 1983 SSC Workshop, Ann Arbor, MI (1983).

82. A. Piwinski, "Beam Height in PETRA," DESY M-83-19 (1983).

83. A. Poncet, "Trapping of Ions in the EPA Electron Beam: Stability Conditions and Diagnosis," CERN PS 88-14 (1988).

84. T. O. Raubenheimer, "Tolerances to Limit the Vertical Emittance in Future Storage Rings," To be published in Particle Accelerators; and SLAC-PUB4937 (1991).

85. T. O. Raubenheimer and R. D. Ruth, "A Dispersion-Free Trajectory Correction Technique for Linear Colliders," Nucl. Instr. Meth., A302, 191 (1991).

86. T. O. Raubenheimer and R. D. Ruth, "A New Trajectory Correction Technique for Linacs," Proc. of the 2nd European Part. Acc. Conf., Nice, France (1990). 
87. T. O. Raubenheimer, "A New Technique of Correcting Emittance Dilutions in Linear Colliders," Nucl. Instr. Meth., A306, 61 (1991).

88. T. O. Raubenheimer, "A New Method of Correcting the Trajectory in Linacs," Proc. of the 1991 IEEE Part. Acc. Conf., San Francisco, CA (1991); and SLAC-PUB-5540.

89. T. O. Raubenheimer, "A Formalism and Computer Program for Coupled Lattices," Proc. of the 1989 IEEE Part. Acc. Conf., Chicago, IL. (1989).

90. T. O. Raubenheimer, L. Z. Rivkin, and R. D. Ruth, "Damping Ring Designs for a TeV Linear Collider," Proceedings of the DPF Summer Study, Snowmass '88, Snowmass, CO (1988).

91. T. O. Raubenheimer, et. al., "A Damping Ring Design for Future Linear Colliders," Proc. of the 1989 IEEE Part. Acc. Conf., Chicago, IL. (1989).

92. L. Z. Rivkin, "Damping Ring of the SLC," Ph.D. Thesis, California Inst. Tech. (1986).

93. K. W. Robinson, "Radiation Effects in Circular Accelerators," Phys. Rev., 111, 373 (1958).

94. J. Rossbach, "Closed-Orbit Distortions of Periodic FODO Lattices due to Plane Ground Waves," Particle Accelerators, 23, 121 (1988).

95. J. Rossbach, "Fast Ground Motion at HERA," DESY 89-23 (1989).

96. R. D. Ruth, unpublished.

97. R. D. Ruth, "Emittance Control in Linear Colliders," Proc. of 1991 Part. Acc. Conf., San Francisco, CA (1991); and SLAC-PUB-5568.

98. R. D. Ruth, "Beam Dynamics in Linear Colliders," Proc. of 1990 Linear Acc. Conf., Albuquerque, NM (1990).

99. R. D. Ruth, "Emittance Preservation in Linear Colliders," Proc. of 1986 US/CERN Part. Acc. School, South Padre Island, TX (1986).

100. R. D. Ruth, "Single Particle Dynamics in Circular Accelerators," AIP Conf. Proc. 153: Proc. of the 1985 US Part. Acc. School, AIP, New York (1987).

101. R. D. Ruth, International Workshop on the Next Generation Linear Colliders, SLAC-335 (1988).

102. F. Sacherer, "RMS Envelope Equations with Space Charge," IEEE Trans. Nucl. Sci., 18, 1105 (1971). 
103. R. Sah, "A Beam-beam Simulation for the Single Pass Linear Collider," SLAC-AATF/80/14 (1980).

104. M. Sands, "The Physics of Electron Storage Rings," SLAC-121 (1971).

105. M. Sands, "Emittance Growth from Radiation Fluctuations," SLAC-AP-47 (1985).

106. J. Seeman, "New Compensation of Transverse Effects in a Linac by Displacing Accelerating Structures,"Proc. of 1990 Linear Acc. Conf., Albuquerque, NM (1990).

107. J. Seeman, M. Ross, J. Sheppard, and R. Stiening, "RF' Beam Deflection Measurements and Corrections in the SLC Linac," Proc. of the 1985 IEEE Part. Acc. Conf., NS-32, 2629 (1985).

108. J. Seeman, "Effects of RF Deflections on Beam Dynamics in Linear Colliders," Proc. XIV Int. Conf. on High Energy Acc., Tsukuba, Japan (1989).

109. J. Schwinger, "On the Classical Radiation of Accelerated Electrons," Phy. Rev., 75, 1912 (1949).

110. W. Spence, private communication.

111. J. Spencer, "High Brightness Sources for Colliders," Proc. of the 1991 Part. Acc. Conf., San Francisco, CA (1991); and SLAC-PUB-5561.

112. T. Suzuki, "Vertical Dispersion Produced by Random Closed Orbit Distortions and Sextupoles," SLAC-PEP-259 (1977).

113. T. Suzuki, "Synchro-Betatron Resonance Driven by Dispersion in RF Cavities," Particle Accelerators, 18, 115 (1985).

114. T. Suzuki, "Synchro-Betatron Resonances," Proc. 14th Int. Conf, on High Energy Accelerators, Tsukuba, Japan (1989).

115. K. A. Thompson, private communication.

116. K. A. Thompson and R. D. Ruth, "Transverse Coupled-Bunch Instabilities in Damping Rings of High-Eilergy Linear Colliders," Phys. Rev., D43, 3049 (1991).

117. K. A. Thompson and R. D. Ruth, "Controlling Transverse Multi-Bunch Instabilities in Linacs of Higl-Energy Linear Colliders," Phys. Rev., D41, 964 (1990). 
118. C. Travier, "RF GUNS: Bright Injectors for FELs," SERA 90-248 (1990).

119. J. Urakawa,et. al. "Preliminary Designs of 1.54 Gev Damping Ring and Bunch Compressor for the JLC," Proc. of the 1990 Linear Accelerator Conf., Albuquerque, NM (1990).

120. H. Wiedemann, "Scaling of FODO Cell Parameters," SLAC-PEP-Note-39 (1973).

121. H. Wiedemann, "Enlargement of the Electron Beam Cross Section due to an Oscillating Synchrotron Radiation Damping Time Constant," SLAC-PEP-. Note-48 (1973).

122. H. Wiedemann, "Scaling of Damping Rings for Colliding Linac Beam Systems," Proc, of the XIth Int. Conf. on High Energy Acc., Geneva (1980).

123. H. Wiedemann, "Beam Spot Size and Tolerances," SLAC-CN-211 (1982).

124. H. Wiedemann, "Low Emittance Storage Ring Design," Proc, of 1986 US/CERN Part. Acc. School, South Padre Island, TX (1986).

125. F. Willeke and G. Ripken, "Methods of Beam Optics," AIP Conf. Proc. 184: Proc. of the 1987 US Part. Acc. School, AIP, New York, (1989).

126. L. H. Y'u, et. al., "Real Time Closed Orbit Correction System," Proc. of the 1989 IEEE Part. Acc. Conf., Chicago, IL (1989).

127. Many of the parameters of these future linear colliclers can be found in: Second International Workshop on Next Generation Linear Colliders, KEK Internal 90-22 (1990); and International Workshop on the Next Gencration Linear Colliders, SLAC-335 (1988).

128. The SLC Design Handbook, R. Erickson Ed., SLAC, Stanford, CA (1986).

129. The ALS is a $1-2 \mathrm{GeV}$ synchrotron light source described in: 1-2 GeV Synchrotron Radiation Source: Conceptual Design Report, LBLPUB-5172 Rev. (1986).

130. Proce dings of the ICFA Workshop on Low Emittance $e^{+} / e^{-}$Bearns, Brook. haven, NY (1987).

131. Proceedings of the ICFA Workshop on Short Pulse High Current C'athodes, Bendor, France (1990).

132. An Asymmetric B Factory: Conceptual Design Report, SLAC-372 (1991). 
133. This is a property of sums of gaussian random variables. For example see: M. Fisz, "Probability Theory and Mathematical Statistics - Third Edition," Wiley, New York (1963), p. 150.

134. Many laboratories are designing damping rings for future linear colliders. Details of the SLAC design can be found in Ref, 90. Other preliminary designs can be found in Ref. 127. 

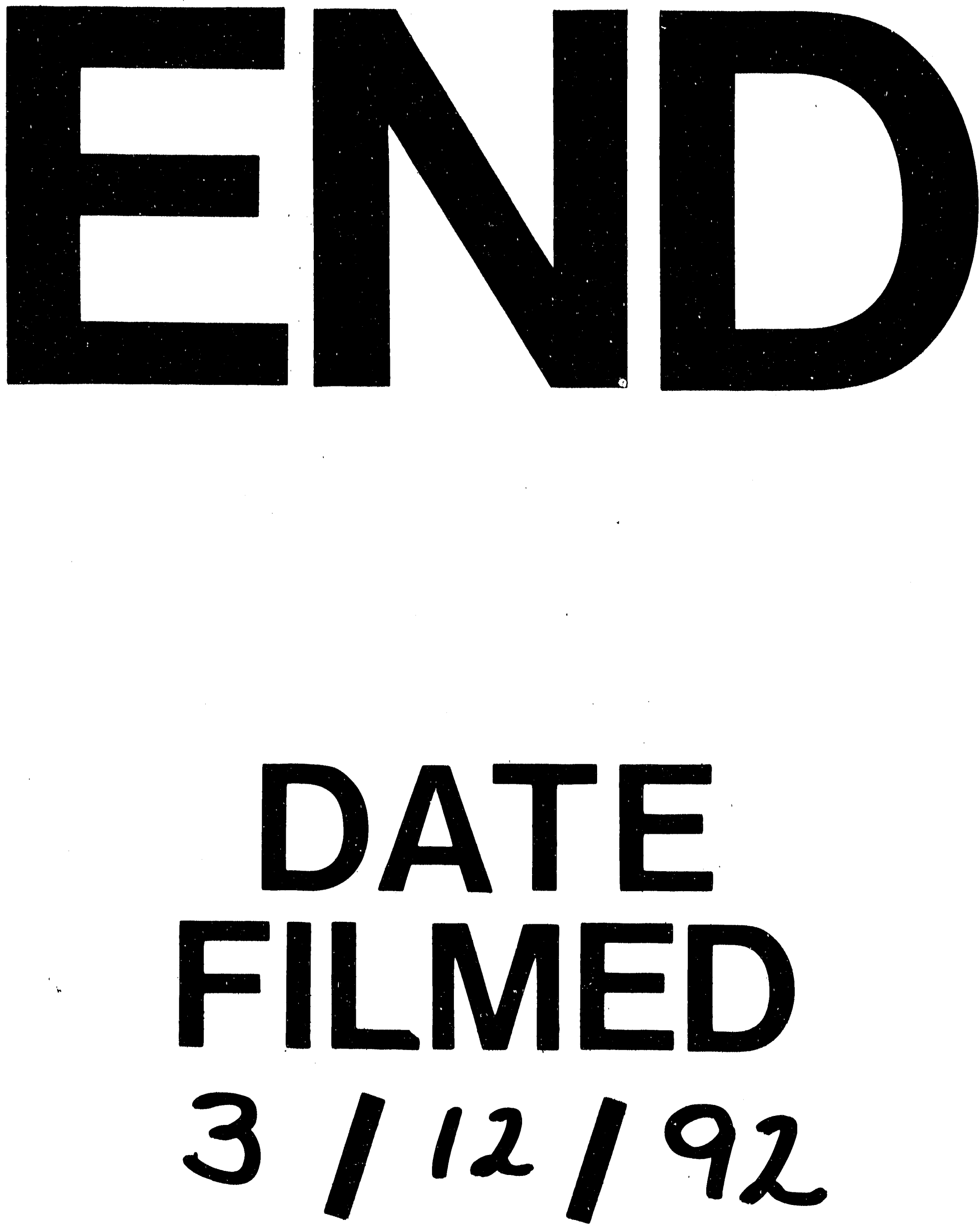


\section{,}

